\title{
Programmed Cell Death
}

S01-01

\section{PROGRAMMED CELL DEATH: A SWISS DISCOVERY}

S. Clarke \& P.G.H. Clarke

Institutes of Physiology, and of Anatomy, University of Lausanne, CH-1005 Lausanne.

On this 25th anniversary of USGEB, in Bern, we point out that research on programmed cell death is much older than current fashion, going back to Carl Vogt, 1842. Born and educated in Giessen, Germany, but of Bernese parents, vogt did a medical doctorate in Bern. He then moved to Neuchâtel, and whilst working there with Agassiz undertook an extensive study of anuran development, published in 1842. Inspired by Schwann, Vogt asked cellular questions, and showed that the well known disappearance of the notochord during climax was due to cell death. During the rest of his long career, spent mainly in Geneva, Vogt did not publish again on cell death, but its importance became clear following reports of Weismann $(1864,1866)$ and Lowne (1870) in insects, and of Goette (1875), Froriep (1882, 1885), Metschnikofe (1883), Mayer (1886) and Barfurth (1887) in vertebrates.

\section{S01-02}

Early changes of membrane signalling enzymes in glucocorticoid-induced apoptosis of human T-cells.

Graber R. and Losa G. A., Laboratorio di Patologia Cellulare, Istituto Cantonale di Patologia, 6604 Locarno, Switzerland.

The behaviour of membrane enzymes PIP2-phospholipase C (PLC) modulating the membrane transduction of extracellular signals and $\gamma$ glutamyltransferase $(\gamma-\mathrm{GT})$ involved in metabolites transport, was followed during the early phase of glucocorticoid $\left(10^{-6} \mathrm{M}\right.$ dexamethasone) treatment leading to apoptosis in CCRF-CEM cells. Apoptosis could be evidenced only after 72 hours of dexamethasone treatment by measuring the decrease of cell number, cellular activation (MTT) and S-phase percentage, the enhancement of DNA fragmentation and uptake of propidium iodide and by ultrastructural modifications of chromatine and cell membranes. At the molecular level however, we could notice a $20 \%$ increase in the activity of $\gamma$-GT and PLC still at 15 and $30 \mathrm{sec}$ respectively upon dexamethasone treatment. Both activities declined to the control level after 5 and $1 \mathrm{~min}$ respectively without changes up to $72 \mathrm{~h}$. Our results indicated that dexamethasone, which usually reaches nuclear DNA after binding to cytosolic receptors, was able to elicit a fast activation of membrane enzymes involved in signal transduction, which by far precceded the cellular events related to the apoptotic death of $\mathrm{CD}^{+} \mathrm{T}$ lymphoblastoid cells.

S01-03

MOTONEURON DEATH INDUCED BY AXOTOMY IS PREVENTED BY bcl-2 PROTO-ONCOGENE IN NEONATAL TRANSGENIC MICE. Dubois-Dauphin* M. Frankowsky H. Huarte J. and Martinou J.C. Dpts of Physiology and Morphology, Univ. Med. Center and Glaxo Imb, Geneva, Switzerland

In rodents, the period of naturally occuring cell death of motoneurons is followed by a period of supersensitivity to axonal injury. Thus, in newborn rodents lesion of the facial nerve leads to a rapid degeneration of the injured motoneurons. We have tested whether overexpression, in vivo, of the bcl-2 proto-oncogene was capable of preventing death of axotomized motoneurons. To address this question we used transgenic mice whose motoneurons overexpress the Bcl- 2 protein. One of the two facial nerves of newborn mice was transected on the 2 nd-3rd post-natal day. Seven days after the lesion, the morphology of the facial nuclei was analyzed. In control mice, and when compared to the intact nucleus, 70 to $80 \%$ of axotomized motoneurons had disappeared. In contrast, in the transgenic animals, the number of motoneurons on the lesioned side remained unchanged when compared to the contralateral nucleus. Furthermore, their axons remained visible up to the distal lesion site. These experiments show that, in vivo, motoneurons overexpressing the $\mathrm{Bcl}-2$ protein survive after axotomy, and suggest that, in vivo, Bcl-2 protect neurons from experimentally induced cell death and could be a target for treatment of motoneurons degenerative diseases.
S01-04

\section{NEURONAL DEATH IN VERTEBRATE DEVELORMENT}

Peter G.H. Clarke and Marie-Pierre Primi Institute of Anatomy, Univ, of Lausanne, CH-1005.

It is widely held that all developmental cell death is of a single type (apoptosis) and that neuronal death is primarily for adjusting the number of neurons in a population to the size of their target field through competition between equals for target-derived factors. We shall draw on our research and on that of others to criticize these views and replace them by the following.

At least three types of neuronal death occur, only one of which resembles apoptosis; a neuron can choose between several self-destruct mechanisms depending on the cause of its death.

The purpose of the death is to regulate connectivity, not neuron number. Competitors for trophic factors are unequal, and many losers have made axonal targeting errors.

A neuron's survival and differentiation depend on multiple anterograde and retrograde signals. Activity affects retrograde signals and some but not all anterograde ones. The pattern of activity is more important than the overall amount.

\section{S01-05}

\section{SECRETION OF CLUSTERIN AND APO E DURING INDUCED CELL DEATH OF CULTURED RAT SPINAL CORD NEURONS}

Messmer S., Mattenberger L., Sagot Y., Blatter-Garin M-C., Pometta D., Kato A., James R.W.

Dépt de Médecine, Dépt. de Pharmacologie, Div. de Neurophysiologie Clinique, Faculté de Médecine, Genève.

Clusterin is a widely expressed glycoprotein, highly conserved across species. Numerous functions have been postulated for this protein. The most important are roles in lipid transport, as clusterin is associated with apolipoprotein AI in HDL, complement regulation and tissue remodelling, in particular during cell death and differentiation.

Using cultures of rat spinal cord neurones $(90 \%$ neurons and 5 $10 \%$ non-neuronal cells), we have studied the expression of clusterin and apo $\mathbf{E}$ in glutamate-induced neuronal cell death to examine potential roles in lipid management. Up-regulation of the two proteins was observed. Clusterin and apo $\mathrm{E}$ appear in the conditioned medium respectively $15 \mathrm{~h}$ and $7.5 \mathrm{~h}$ after incubation with glutamate. Control studies, in the presence of a noncompetitive NMDA receptor agonist showed the secretion of clusterin and apo $\mathrm{E}$ to be diminished by $>60 \%$. No up-regulation of either protein was observed in complementary studies with exclusively non-neuronal cell cultures. The cellular origin of the 2 secreted proteins is presently under investigation.

\section{S01-06}

\section{ISOLATION OF GENES REGULATED DURING PROGRAMMED CELL DEATH AND TISSUE REMODELLING IN MAMMARY AND PROSTATE GLANDS}

W. Bielke, K.Guo, S. Saurer and R. R. Friis; University of Bern, Laboratory of Clinical and Experimental Research, Tiefenaustr.120, 3004 Bern

Programmed cell death and tissue remodelling are consequences of hormonally induced restructuring of the rat ventral prostate after castration and the rat mammary gland after weaning. We used the "Differential Display"-method (Liang and Pardee, 1992, Science 257:967) to detect and isolate cDNA fragments whose corresponding RNAs are regulated either coincidentally, or in an organ specific fashion during mammary gland involution and postcastrational prostate regression. Partial sequencing of 12 clones revealed high, but not absolute homology of 5 fragments with sequences, previously characterized in different biological contexts. These five encode functions which could be anticipated to be important for cell growth and/or programmed cell death. We are presently investigating the functions of several of these transcripts in cell culture and in vivo. Antisense oligos are being employed in vivo to determine whether these genes contribute to the phenotype of programmed cell death. 
S01-07

CONSTRUCTION OF INERT AND LIVE VACCINES CARRYING MOUSE MAMMARY TUMOR VIRUS ENVELOPE AND SUPERANTIGEN EPITOPES

Astori M. Hopkins S.A. Karapetian O., Acha-Orbea H. and

Institute of Biochemistry, University of Lausanne, and Swiss Institute for Experimental Cancer Research, CH-1066 Epalinges.

B epitopes derived from the envelope gp52 glycoprotein (EP3) or from the viral superantigen of MMTV have been incorporated into inert or live vaccines. The inert vaccine consists of purified chimeric proteins which contain the B epitopes alone or fused to multimeric promiscuous T helper epitopes from tetanus toxin. Mice were immunized subcutaneously with these chimeric proteins. The live vaccine consists of an avirulent strain of Salmonella typhimurium which expresses the MMTV epitopes in the form of chimeric proteins fused to the nucleocapsid protein of Hepatitis B Virus. This vaccine is given to mice in one oral dose. The level, duration and isotype of the immune response generated by each vaccine have been measured and compared. The level of protection has been investigated by systemically challenging immunized mice with the retrovirus.

\section{$501-08$}

TESTOSTERONE TREATMENT OF SENESCENT RATS RESTORED OXYTOCIN BINDING SITES IN SPECIFIC AREAS OF THE BRAIN, BUT NOT IN THE STRIATUM.

Arsenijevic Y., Dreifuss J.J. and Tribollet E., Department of Physiology, Centre Médical Universitaire, CH-1211 Geneva 4.

A reduced binding of oxytocin (OT) occurs with aging in some, but not all, areas of the rat brain (Arsenijevic et al, Experientia 1993, 49 A75). The caudate putamen showed the most impressive loss of OT receptors. Two other regions, the hypothalamic ventromedial nucleus (VMH) and the islands of Calleja (ICj) had also an important deficit of OT binding sites. On the other hand, these two regions were known to be sensitive to sex steroids. In the present work, we treated from 20 month old rats during one month with testosterone propionate $(2 \mu \mathrm{g} / \mathrm{kg}$ s.c., once every 3 days) dissolved in oil. Three rats of the same age injected with oil only served as controls. We labelled OT receptors throughout the brain of old rats using a ${ }^{125}$ I-labelled ligand specific for OT receptors. Analysis of autoradiograms by an image analyzer revealed that the testosterone treatment increased $O T$ binding sites in the VMH, in the ICj, and, to a lesser extent, in the bed nucleus of the stria terminalis, a region also sensitive to sex steroids. By contrast, in the caudate putamen, the disappearance of OT receptors was not compensated. In conclusion, the decrease of OT receptors occurring in VMH and ICj with aging can be reversed by administration of gonadal steroids. In contrast, the loss of OT receptors in the striatum appears to depend on another mecanism.

S01-09

EXPRESSION OF VASOPRESSIN RECEPTORS WITHIN MOTOR NUCLEI FOLLOWING PERIPHERAL NERVE SECTION IN THE ADULT RAT.

Tribollet E., Marguerat A., Dreifuss J.J. and Arsenijevic Y. Department of Physiologie, CMU, 1211 Geneva 4

Vasopressin (AVP) receptors are expressed transiently in the facial nucleus during development (Tribollet et al., 1991, Dev. Brain Res., 58, 13-24). AVP may therefore play a role in the maturation of neuromuscular connexions in the neonate rat, and possibly in the restauration of these connexions after nerve lesion in the adult. In order to investigate the latter proposition, we have sectionned the facial nerve in adult rats and used quantitative autoradiography to look at AVP binding sites in the facial nucleus at various postoperative times. We observed a massive and transient increase of AVP binding sites on the operated side. The number of facial AVP binding sites reaches a maximum about one week after nerve section, remains stable during 23 weeks, then begin to decrease towards control level. The induction of AVP receptors is markedly delayed if the proximal stump of the nerve is ligated. To assess whether other motor nuclei would also react to axotomy by up-regulating the expression of AVP receptors, we have sectionned the hypoglossal nerve and the sciatic nerve. In both cases, the binding of AVP receptor ligand increases massively in the respective motor nuclei, with a time-course similar to that found in the facial nucleus. Altogether, our data suggest that central AVP could be involved in the process of nerve regeneration.
S01-10

CYTOTOXIC T-CELL MEDIATED APOPTOSIS

Schaerer, E, Karapetian, O., Adrian, M. and Tschopp, J. Inst. de Biochimie, Univ. de Lausanne, 1066 Epalinges.

An apoptotic celi death mechanism is used by cytolytic T cells (CTL) to lyse appropriate target cells. CTL harbox cytoplasmic storage compartments, containing the lytic protein perforin and serineproteases (granzymes), whose content is released upon target cell interaction. We show that these granules are multivesicular bodies and that degranulation releases these intragranular vesicles (IGV) having granzymes, T-cell receptor and yet undefined proteins associated. Isolated IGVs and perforin induce DNA breakdown in target cells within 20 minutes. Microscopic analysis demonstrates that IGV specifically interact with target cell via the T-cell receptor and that their contents is taken up by the target cell. Already 15 min. after interaction, 3 distinct IGV proteins are Found in the nucleus of the target cell. One of the molecules has been identified to be granzyme $A$ previously reported to be involved in apoptosis. We propose that lymphocytes transfer apoptosisinducing proteins to the nucleus of the target cells using vesicles as vehicles for delivery.

\section{S01-11}

TIA, A NOVEI T CELL-ASSOCIATED PROTEIN: ITS ROLE IN APOPTOSIS

Bente Lowin*, Lars Erench\$, Jürg Tschopp*,

Institute of Biochemistry, University of Lausanne, Epalinges, Switzerland, \$ Department of Immunonephrology, CMU, Geneva, Switzerland

Cytotoxic T cells kill their targets by a mechanism involving membranolysis and DNA degradation (apoptosis). Recently, two sets of proteins have been proposed as DNA breakdown-inducing molecules in $T$ cells: granzyme $A, B$ and $T I A-1$. In this study we cloned and further characterized the TIA-1 mouse homologue. Aa sequence comparison with the human TIA-1 showed an overall identity of $93 \%$. Devoid of a signal peptide, TIA is yet localized to cytotoxic granules, probably targeted via a Gly-Tyr-motif. As TIA-1, its mouse homologue contains three RNAbinding domains. Expression of TIA during development shows a very strong signal in the brain and weaker signals in thymus, heart and other organs. During embryonic development several structures that contribute to organogenesis form transiently and are later eliminated by apoptosis. This pattern of TIA expression could indicate its involvement in apoptosis.

\section{S01-12}

PROGRAMMED CEIL DEATH IN THE PROSTATE FOLIOWING CASTRATION: CIONING OF A TESTOSTERONE-DEPENDENT NADH DEHYDROGENASE GENE Guo Ke, W. Bielke, S. Saurer, V. Merz, R. Ball and R. Friis, Univ. Berne, Lab for clin.-exp. Rés., Tiefenaustr. 120, Bern

Prostate involution occurs after castration in rats and is associated with the death by apoptosis of a large fraction of the epithelial cells. We have isolated several genes from a prostate involution bacteriophage lambda library using differential screening methods. Among these clones, one demonstrated an especially strong signal when used as a probe against Northern blots of prostate mRNA obtained before, and at different times after castration. This gene is down-regulated after castration by 40 -fold within 5 days. Intramuscular injection of a testosterone depot resulted in complete restoration of expression within 24 hours. Upon sequencing it became apparent that this clone has a high degree of homology to a known NDAH dehydrogenase encoded in mitochondrial DNA. The clone failed to hybridize to any transcripts from rat organs other than prostate. We are now in the process of isolating the human homolog to this gene for use as a bianarker in study of benign hyperplasia and developing carcincma. This gene is a possible indicator for testosterone-independent cell populations or of cells lacking functional testosterone receptor. 
S01-13

REMODELING OF LUNG STRUCTURE DURING POSTNATAL CELL DEATH

Schittny, J.C. and Burri, P.H. Institute of Anatomy, University of Bern, CH-3000 Bern 9, Switzerland

During the first three postnatal weeks the rat lung undergoes the last two developmental stages, the phase of alveolarization and the phase of microvascular maturation. The latter involves a decrease of the connective tissue mass in the alveolar septa and a merging of the two capillary layers to a single one.

Speculating that programmed cell death may play a role during this remodeling, we searched for the presence of apoptotic cells in rat lungs between days 10 and 24 . Lung paraffin sections were treated with 3'-terminal transferase, digoxigenin-dUTP, and anti-digoxigeninfluorescein- $F(a b)$-fragments, and the number of fluorescent nuclei was compared between sections at different days. While the number of apoptotic cells was low until the end of the second week and at day 24 , we observed an about eight fold increase of fluorescent nuclei towards the end of the third week. We conclude that programmed cell death is involved in the structural maturation of the lung.

\section{S01-14}

THE GIANT LENS LOCUS IS INVOLVED IN CELI FATE REGULATION AND AXON GUIDANCE IN THE VISUAL SYSTEM OF DROSOPHILA

Brunner, A., Wallrapp, Ch.,Pollack, I, Twardzik, $\mathrm{T}$. and Schneuwly, S.

Lehrstuhl Genetik, Biozentrum

Universität Würzburg, D-97074 Würzburg

Mutants in the giant lens (gil) gene show a strong disturbance in ommatidial development. In the absence of any gene product, additional photoreceptors, cone cells and pigment cells develop. Opposite effects can be seen in flies in which the gene product of the giant lens gene can be ectopically expressed by heat shock. A second very typical phenotype is the disturbance of photoreceptor axon guidance. Molecular analysis of gil shows that it encodes a secreted protein of $444 \mathrm{a} a$ containing three evolutionary conserved cystein-motives very similar to EGF-like repeats. we propose that $g i l$ functions as a secreted signal, most likely a lateral inhibitor for the development of specific cell fates and that gil, either directly or indirectly, is involved in targeting photoreceptor axons into the brain.

\section{S01-15}

THE DECREASE IN CELLULARITY DURING SCAR ESTABLISHMENT IS MEDIATED THROUGH APOPTOSIS

DESMOULIERE, A., REDARD, M., DARBY, I., AND G. GABBIAN

Department of Pathology, CMU, 1 rue Michel Servet, 1211 Genève 4

During the healing of an open wound, granulation tissue formation is characterized by replication and accumulation of fibroblastic cells, many of which acquire morphological and biochemical features of smooth muscle cells and have been named myofibroblasts (Schürch et al., Histology for Pathologists, 1992). As the wound evolves into a scar, there is an important decrease in cellularity, including disappearance of myofibroblasts. The question arises as to which process is responsible for myofibroblast disappearance.

During a previous investigation on the expression of $\alpha$-smooth muscle actin in myofibroblasts, we have observed that in late phases of wound healing, many of myofibroblasts show signs of apoptosis and suggested that this type of cell death is responsible for the disappearance of myofibroblasts (Darby et al., Lab. Invest. 63:21, 1990). We have tested this hypothesis by means of electron microscopy and morphometry and by in situ end-labeling of fragmented DNA (Wijsman et al., J. Histochem. Cytochem. 41:7, 1993). Our results show that the number of apoptotic cells increases as the wound closes and suggest that this may be the mechanism for the disappearance of myofibroblasts as well as for the evolution of granulation tissue into a scar. (Supported by the Swiss National Science Foundation, Grant $n^{\circ} 31-30796.91$ )
S01-16

\section{PRO'TEIN KINASE A AND AP-1 (cFOS/JUND) ARE INDUCED DURING PROGRAMMED CELL DEATH OF MOUSE}

\section{MAMMARY EPITHELIAL CELLS}

R. Jaggi, A. Marti and B. Jehn. Universität Bern, AKEF, Tiefenaustr. 120, 3004 Bern

At weaning the mammary gland undergoes a reductive remodelling process (involution) which is associated with the cessation of milk protein gene expression and apoptosis of milk-producing epithelial cells. This process can be reversed by returning the pups to the mother within 1 day. Elevated nuclear protein kinase A (PKA) activity was observed from one day post-lactation, paralleled by increased $c-f o s, j u n \mathrm{~B}, j u n \mathrm{D}$ and to a lesser extent c-jun mRNA levels. AP-1 DNA binding activity was transiently induced and the AP-1 complex was shown to consist principally of cFos/JunD. Oct-1 DNA binding activity and Oct-1 protein were gradually lost from the gland over the first four days of involution, whereas Oct-1 mRNA levels remained unchanged. Comparing nuclear extracts from normal mammary glands with nuclear extracts from glands which had been cleared of all epithelial cells three weeks after birth revealed that PKA activation, AP-1 induction and Oct-1 inactivation are all dependent on the presence of the epithelial compartment. The increased Fos/Jun expression and the inactivation of Oct-1 may be consequences of the increased PKA activity. When involution is reversed, both, PKA activity and AP-1 DNA binding activity (and fos and jun mRNA levels) are reduced to basal levels. Our data suggests a role for PKA and AP-1 on programmed cell death of mammary epithelial cells.

\section{S01-17}

Bcl-2 $\alpha$ does not require membrane attachment for its survival activity

C. Borner*, I. Martinout, C. Mattmann*, M. Irmler*, E. Schärrer ${ }^{*}$, J.-C. Martinout, and J. Tschopp*. * Institute of Biochemistry, University of Lausanne, 1066 Epalinges, $†$ Institute of Molecular Biology, Glaxo Inc., 1228 Plan les Ouates.

$\mathrm{Bcl}-2 \alpha$ is a mitochondrial or perinuclear-associated oncoprotein that prolongs the life span of a variety of cell types by interfering with programmed cell death. How it exerts this activity is unknown but it is believed that membrane attachment is required. To identify critical regions in bcl- $2 \alpha$ for subcellular localization and survival activity, we created by site-directed mutagenesis, various mutations in regions which are most conserved between the different bcl-2 species. We show here that membrane attachment is not required for the survival activity of bcl-2 2 . A truncation mutant of bcl-2 $\alpha$ lacking the last 33 amino acids (T3) including the hydrophobic domain is soluble, yet fully active in blocking apoptosis of sympathetic neurons induced by NGF deprivation or L929 fibroblasts induced by TNF $\alpha$ treatment. We further provide evidence for a putative functional region in bcl-2 which lies in the conserved domains 4 and 5 upstream of the hydrophobic $\mathrm{COOH}$ terminal tail.

S01-18

LAMIN BREAKDOWN DURING APOPTOSIS ALLOWS DNASE I TO ENTER. THE NUCLEUS LZADING TO DNA BREAKDOWN

M.Schröter, M.Peitsch, P.Gallant*, E.A.Nigg*,

J.Tschopp

Université de Lausanne, Institut de Biochimie/

* ISREC, Ch. des Boveresse 155, CH-1066 Epalinges

The breakdown of nuclear DNA is considered to be a hallmark of apoptosis. We previously identified the perinuclear membrane localized DNase I as the endonuclease involved in the formation of oligonucleosomal-sized fragments (DNA ladder). It is not clear how the nuclease is activated and has access to the DNA. We show that in thymocytes induced to undergo apoptosis, lanin breakdown preceded DNA laddering. BY transfecting HeLa cells with a constitutively active cdc2 mutant, nuclear envelope breakdown and typical apoptotic features (chromatin condensation) were observed. Moreover, co-transfection with cdc2 mutant and DNase I led to DNA degradation. We propose that apoptosis can be induced by wrongly timed and hence abortive mitosis leading to uncontrolled nuclear membrane disintegration. 


\section{Respiratory Disease Models}

S02-01

STEREOLOGICAL ASSESSMENT OF PLATELET-DERIVED GROWTH FACTOR IMMUNOREACTIVE CELLS IN HUMAN LUNGS WITH BRONCHIOLITIS OBLITERANS-ORGANIZING PNEUMONIA

Aubert JD', Hayashi $\mathrm{S}^{2}$, Paré $\mathrm{PD}^{2}$, and $\mathrm{JC}$ Hogg ${ }^{2}$

1Pulmonary Division, CHUV, Lausanne. ${ }^{\mathrm{UBBC}}$ Pulmonary Research Laboratory, St Paul's Hospital, Vancouver BC, Canada.

Platelet-derived growth factor (PDGF) is thought to play an active role in fibrosing diseases. Bronchiolitis obliterans-organizing pneumonia (BOOP) is a condition characterized by intraluminal proliferation of connective tissue inside distal air spaces. To evaluate PDGF expression in BOOP we performed immunohistochemistry on lung biopsies from 20 patients and 10 controls free of fibrosis. Serial sections were stained with an antibody against either PDGF or the monocyte/macrophage marker CD68. In both groups the PDGF $\oplus$ cells were essentially tissue macrophages. Using point counting to measure volume fraction ( $V_{V}$ ), PDGF-positive cells represented $4.65 \pm 1.63 \%$ (mean $\pm S D$ ) of the volume occupied by lung tissue in the BOOP cases, and $2.12 \pm 0.65 \%$ in the controls $(p<0.001)$. Similarily, $10.73 \pm 4.69 \%$ of the lung tissue was occupied by CD68 $\oplus$ macrophages in the BOOP cases, compared to $5.37 \pm 3.73 \%$ in the contrals $(p<0.005)$. A positive linear correlation was found between the $V_{V}$ of PDGF $\oplus$ cells and the $V_{V}$ of $C D 68 \oplus$ cells on the 30 cases $(r=0.50 \quad p<0.005)$. We conclude that $B O O P$ is characterized by a recruitment of tissue macrophages and that a significant proportion of them express PDGF. As PDGF can also induce the macrophage chemo-attractant $M C P-1$, we speculate that macrophages are involved in a positive feed back regulation, and may play a pivotal role in the pathogenesis of BOOP.

\section{$\mathrm{S} 02-02$}

CONSTITUTIVE INOS EXPRESSTON IN A BRONCHIAL EPITHELIAL CELI ITNE.

Felley-Bosco E.*, Ambs S.*, Lowenstein C.J.\#, Snyder S.H.\# and Harris C.C., *Laboratory of Human Carcinogenesis, NCI, Bethesda MD 20892*, \#Johns Hopkins University School of Medicine, Baltimore, MD 21205 .

Two major roles have been defined for No: cellcell communication mediated by the stimulation of CGMP synthesis, and cytotoxicity by direct interaction of the free radical No with cellular target. To investigate these effects in human epithelia, which constitute a major source of No in man, we introduced the murine inos sequence into SV40-T immortalized human bronchial epithelial cells (Beas-2B). iNos activity was higher than 100 pmol citrulline/min/ mg prot in the first passages of iNos transfected cells, but it decreased by subculturing. No iNOS activity was detected in cells transfected with control vector. In iNos transfected cells, No stimulated a CGMP synthesis and c-fos expression which could be inhibited by addition of LNMA. Thus, in human epithelial cells No is able to significantly alter signal transduction pathway.

\section{S02-03}

\section{DIFFERENTIAL DISPLAY OF GENES REGULATED DURING COMPENSATORY GROWTH OF THE LUNG AFTER PNEUMONECTOMY \\ C. Vallan, W. Bielke*, B. Friis* and P. Burri}

Institute of Anatomy and AKEF ; University of Bern; CH-3009

Partial pneumonectomy, i.e. the resection of lung tissue, is known to initiate compensatory growth in the remaining lobes. Under appropriate conditions this results in a complete restoration of lung parenchymal structure and function. Unfortunately the molecular mechanisms controlling the onset of this compensatory growth are poorly understood. The aim of our study was to find and characterise genes which are up or down regulated during this time.

For isolating such genes, we chose the differential display approach (P.Liang and B.Pardee 1992, Science 257:p 967). Therein a 3'end fragment of a subset of reverse transcribed mRNAs is amplified by the polymerase chain reaction. The fragments can be separated on a DNA sequencing gel. Genes differentially expressed in pneumonectomised and sham-treated rats show different patterns on these gels. The fragments of such genes can be isolated from the gel and cloned into vectors in order to provide tools to characterise their gene. A set of such genes, some up-regulated and others downregulated following pneumonectomy, have been isolated, partially sequenced and characterised.
S02-04

\section{BARRIER LESIONS IN HYDROSTATIC PULMONARY EDEMA}

D.Wu, H.Bachofen, U.Gyger and E.R. Weibel

Department of Anatomy, University of Bem, CH 3012, Bem

Pulmonary edema induced in isolated perfused rabbit lungs by moderate elevation of perfusion pressure (14 torr ) results in the formation of characteristic bleb-like extensions of the thin cytoplasmic leaflet of alveolar epithelium ( Bachofen et al. Am Rev Resp Dis. Vol.147. pp989-996,1993). In this study, we show by means of electron microscopy and morphometry that the frequency of the blebs correlate with the distribution of alveolar edema fluid. We find that $5 \%$ of the epithelial surface is involved in the formation of blebs. Three-dimensional reconstruction showed that blebs had near-spherical shape and that their opening toward the interstitium is irregular. These finding demonstrate a great plasticity of epithelial cell extensions when subjected to moderate pressure stress. At high pressure the cell linings demonstrate clear breaks.

S02-05

PERFUSION PATTERN OF LUNG MICROVESSELS AFTER SHORT CIRCULATION PERIODS

M.F. König, S. Batra, and E.R. Weibel

Department of Anatomy, University of Berne,

CH-3012 Berne, Switzerland

Recently, we found that under in vivo conditions the whole pulmonary capillary bed in rabbits has been perfused within a time interval of two minutes. In the present study we examined shorter time periods. Our new plasma marker consisting of highly concentrated gold nanospheres was infused via a femoral vein catheter into the right atrium of anaesthetised and ventilated rabbits. Five or 10 seconds after start of infusion, the blood flow was stopped and the lung was fixed by instillation. Silver enhanced paraffin sections revealed labeled and unlabeled blood vessels. Electron-microscopic sections showed various plasma gold particle concentration. Morphometric analysis of electron micrographs showed an increase of the portion of marked capillaries from shorter to longer circulation times. Based on our results, we conclude the existance of inhomogeneous plasma flow velocities within the pulmonary microvascular bed. (Supported by SNSF grant 31-30946.91) 


\section{Mechanisms of Homologous Recombination: Fundamental and Applied Aspects}

S03-01

Structure-function relationships in the multi-functional DNA strand exchange and homologous pairing protein SEP1 from S.cerevisiae

Heidrun Interthal, Vladimir Bashkirov, Ursula Steinmann, Jachen Solinger, Henri Kester, and Wolf-Dietrich Heyer. Institute of General Microbiology, University of Bern, Baltzer-Strasse 4, 3012 Bern.

Hybrid DNA is a central intermediate in homologous recombination in eukaryotes and we have chosen a biochemical approach to identify and characterize proteins that catalyze the formation of hybrid DNA. The S.cerevisiae SEPI protein efficiently catalyzes the formation of hybrid DNA in vitro. In addition, the protein exhibits exonuclease activity on single-stranded and double-stranded DNA, on single-stranded RNA as well as RNaseH activity. Cells lacking this protein exhibit pleiotropic phenotypes including pachytene arrest in meiosis and a decrease in commitment to meiotic intergenic recombination (cross over), In addition, various cellular processes related to mictotubule function like sensitivity to the microtubule drug benomyl, spindle-pole body separation, nuclear migration, and karyogamy are affected. We are using a combination of biochemistry and molecular genetics to determine the structure-function relationships in this large, multi-functional protein to elucidate its in vivo function(s).

\section{S03-02}

Saccharomyces cerevisiae Rad54, a putative DNA helicase involved in recombinational repair and homologous recombination Beate Clever, Jacqueline Schmuckli, and Wolf-Dietrich Heyer. Institute of General
Microbiology, University of Bern, Balizer-Strasse 4, 3012 Bern, Switzerland

Radiation sensitivity in the yeast $S$. cerevisiae is under the control of more than 90 genes They may be arranged in 3 epistasis groups. The $R A D 3$ group ( $R A D 1,2,3,4,7,10,16$ etc.) includes genes involved in the excision repair. The RAD6 group (RADS, 6, 9, 15, I8 etc.)
includes genes involved in error-prone repair and the RADS2 group (RADSO-57, XRS2) are involved in recombinational repair.

RAD54 has been cloned and sequenced (Emery et al, 1991, Gene 104:103-106) Mutations in this gene cause extreme X-ray sensitivity (Game and Mortimer, 1974 Mutation Res, 24:281-292), the inability to repair double-strand DNA breaks (Budd an Mortimer, 1982, Mutation Res, 103:19-24) and decreased spontaneous and induced mitotic recombination (Saeki et al., 1980, Mutation Res. 73: 251-265). Sequence homologies have been reported recently between the Rad54 and a number of other yeast and higher eukaryotic proteins. Rad54 shares homology with the open reading frames encoded by RADS, RAD

The observed bomologies with known helicases suggest that these proteins may represent a new subfamily of potential DNA helicases (Gorbalenya et al., 1989. Nucl. Acids Res 17:47. -4730 . None of the proteins of the new group of potential helicases has been purified and characterized yet. Our primary target is now to purify the Rad54 protein as the first one of this new helicase group. We constructed an overexpression vector which contains the RAD54 gene under the control of the GALIO promoter. The complementation
of a yeast strain mutant for RAD 44 with this overexpression vector was successful, We used of a yeast strain mutant for RADS4 with this overexpression vector was successful. We used the sensitivity to methyl methanesulfonate (MMS), an $X$-ray mimetic drug, as the relevant phenotype. In parallel the gene will also be expressed in $E$. colt as a glutathione $S$ used for the production of polyclonal antibodies. In addition, we are characterizing a temperature-sensitive allele of the RAD 54 gene which might help identify critical tegions of the protein in conjunction with further mutational studies of the protein

\section{$503-03$}

Cloning and characterization of the mouse homolog to S.cerevisiae SEP1 encoding a DNA strand exchange and homologous pairing protein

Vladimir Bashkirov and Wolf-Dietrich Heyer. Institute of General Microbiology, University of Bern, Baltzer-Strasse 4, 3012 Bern.

Homologous pairing and DNA strand exchange are the central steps postulated in the current models of genetic recombination. To date no mammalian gene encoding a homologous pairing protein has been cloned. To get insight into the mechanism of recombination in higher eukaryotes we were interested to clone such genes. Protein sequence comparison of the S.cerevisiae homologous pairing protein, SEP1, and its homolog from $S$.pombe, p140exo2, revealed several conserved stretches of amino acids in their $\mathrm{NH}_{2}$-terminal regions. Using fully degenerate primers and mouse testes CDNA as a template for the polymerase-chain-reaction a product with the expected length has been amplified. This 360 bp DNA was shown to encode a putative polypeptide with high degree of homology to both proteins from the two yeasts. The mouse PCR-product was used as a proteins from the two yeasts. The mouse PCR-product was used as a several rounds of successive screening a total of about $4 \mathrm{~kb}$ of mouse cDNA (mSEP1) was cloned and sequenced. The putative translation product revealed high homology throughout the entire sequence to its S.pombe and S.cerevisiae counterparts, $38 \%$ and $\mathbf{4 1} \%$ of amino acid identity, respectively. The mSEP1 sequence was shown to be unique in the genome and the chromosomal gene is likely to contain many introns. We want to establish a functional full length CDNA clone of MSEPl and characterize the gene and protein product.
S03-04

\section{ORGANIZATION OF MEIOTIC CHROMOSOMES IN S. POMBE}

Bähler J., Scherthan $H^{*}$, and Kohli J., Institute of General Microbiology, Baltzer-Str. 4, 3012 Bem, "Division of Human Genetics and Human Biology, Kaiserslautem.

In Schizosaccharomyces pombe pairing of meiotic chromosomes is not accompanied by the formation of a tripartite synaptonemal complex (SC), a structure which connects the homologs in most other eukaryotes. Meiotic nuclei revealed novel structures ("linear elements") that might be related to the axial elements ( $\mathrm{SC}$ precursors) of other organisms and function in meiotic chromosome pairing, recombination, and segregation (Bähler et ai. (1993) JCB 121:241). To get further insight into the behaviour of meiotic chromosomes, we performed fluorescence in situ hybridizations on spread nuciei with probes from different chromosomal regions. Interestingly, all centromeres and telomeres become clustered during meiotic prophase. The centromeres are paired aiready before meiosis, whereas the telomeres initiate pairing specifically during meiotic prophase, followed by pairing of interstitial regions. Painting of whole chromosomes revealed that the homologs occupy together a specific territory in the nucleus.

\section{S03-05}

ANTISENSE INHIBITION OF HOMEOBOX GENE XHOXIA IN XENOPUS DEVELOPMENT

A. Nichols*, E. Rungger-Brăndle\#, L. Muster*, \& D. Rungger*

*Animal Biology, University of Geneva, $\mathrm{CH}-1224$ Chêne-Bougeries, and \#Electron Microscopy, University Eye Clinic, $\mathrm{CH}-1211$ Geneva 4, Swizzerland

An expression vector containing the VAl gene read by RNA polymerase III, injected into Xenopus zygotes, yields high levels of RNA in virtually all embryonic cells. Anti sense RNA is produced from a segment of the Xhox $1 \mathrm{~A}$ gene inserted in opposite orientation into the VAl gene. Immunological staining of whole mount embryos shows that target mRNA level and $X$ hox $1 \mathrm{~A}$ protein expression of Xhox1A protein is diminished in about half of the antisense treated individuals. Accordingly, nearly half of the embryos develop typical malformations in regions where the Xhox $1 A$ gene is normally expressed. Somites of the anterior trunk are heavily disorganized and mesodermal derivatives surrounding the intestinal tract are reduced or missing. Antisense inhibition, through production of RNA in situ from expression vectors, thus represents a useful tool to study the functional role of zygotic genes in Xenopus development.

\section{S03-06}

DNA RECOMBINATION IN A MAMMALIAN CELL FREE SYSTEM R. Jessberger, H.-R. Rodewald, and Brigitte Riwar; Basel Institute for Immunology, CH 4005 Basel, Switzerland

We have earlier developed a cell free system to study recombinational repair of DNA double strand breaks and deletions. The method allowed us to purify and characterize a high molecular weight multiprotein complex (RC-1), which catalyzes DNA recombination. A DNA polymerase and DNA ligase as well as other enzymatic activities copurify with RC-1. We have commenced an investigation of homologous DNA recombination in nuclear extracts which were prepared from normal and mutated mouse thymocytes. The scid mutation in mice causes an aberrant $V(D) J$ joining reaction, an elevated sensitivity to ionizing radiation and a defect in recombinational repair. Moreover, the normal mouse thymocytes were sorted into the $\mathrm{CD} 4 / \mathrm{CD} 4$ double negative (DN), double positive (DP), and single positive (SP) subclasses and their nuclear extracts, and extracts from RAG-2 \% mice, were tested. Only the scid mice and the CD4/CD8 SP thymocyte extracts were inactive in the recombinational repair reaction. This indicates a development stage specific activity and a scid specific defect of recombinational repair in thymocytes. Restoration of the recombination activity was observed in thymocyte extracts derived from scid, which express a transgene for the $T$-cell receptor $\beta$ chain. A protein could be purified from normal thymus cells to near homogeneity which specifically rescues the recombination activity in scid extracts. This protein, SRSP (Scid Recombination Stimulatory Protein), migrates as a single band of app. $72 \mathrm{kDa}$ in SDS polyacrylamide gel electrophoresis. 
S03-07

PROMOTER DELETION EFFECT ON ADE6-M26 RECOMBINATION ACTIVITY M. Zahn-Zabal and J. Koh1i, Universität Bern

The ade6-M26 mutation in the ade6 gene of the fission yeast Schizosaccharomyces pombe gives rise to a hot spot for meiotic homologous recombination. To address whether transcription plays a role in $M 26$ activity, the effect of the deletion of the ade6 promoter in combination with either the $M 26$ and M375 mutations on recombination frequency was studied at the tetrad level. Deletion in cis of the promoter abolishes M26 hot spot activity relative to the M375 control deletion strain. It is possible that deletion of the promoter has eliminated a sequence necessary for M26 hot spot activity, rather than lack of transcription being responsible for the loss of M26 activity. The role of transcription in $M 26$ hot spot activity is currently being examined.

\section{S03-08}

In vitro hybrid DNA formation by proteins from Schizosaccharomyces pombe E. Käslin and W.-D. Heyer, Inst. für allg. Mikrobiologie, Baltzer.-Str. 4 3012 Bern

Strand exchange and hybrid DNA formation are central steps in the process of homologous recombination. These steps are thought to help in the recognition of homologous DNAs. In yeast, hybrid DNA has been shown genetically as well as physically to occure during homologous recombination. We are interested in the nzymmatic of this process.

Using linerar duplex and circular single-stranded DNA as model substrates, we purified an activity from S.pombe that performes hybrid DNa formation in vitro. This three has been studied extensively ${ }^{1}$ ), the central recombination protein in this organism. The s.pombe activity consists of two components: The first protein, pi $140^{e \times 02}$, is a $140 \mathrm{kDa}$ polypeptide, which has been purified previously from meiotic cells as an exonuclease? This protein is most likely the homologous activity to the Saccharomyces cerevisiae strand exchange protein . $\mathrm{EPPI}^{3}$ The genes of the two proteins are more that $40 \%$ identical and more than $60 \%$ similar. Additionally the two proteins share differen biochemical properties as exonuclease activity on double-stranded and single-stranded DNA, RNAase and RNAaseH activity, and in vitro strand exchange activity, whereas this activity is lower in the case of p140exo2. The second protein, p190/210, consists of a doublet of $190 \mathrm{kDa}$ and $210 \mathrm{kDa}$ on a SDS-PAGE. These two proteins stimulate p $140^{e x 02}$ in the strand exchange assay about 10 fold but have no strand exchange or exonuclease activity on its own. In contrast p190/210 has strand exchange activity resected substrate. We identified p190/210 as fatty acid synthase. Since we cannot rationalize a role for fatty acid synthase in DNA methabolism or recombiantion, this observation casts strong doubts on the specificity of the assay used in this work. for review see: Kowalczykowski S. C. Annu. Rev. Biophys, Biophys, Chem 1991. 20:539-75

2. Szankasi and Smith, Biochemistry, 1992,31,6769-6773

3 Tishkoff et al., Mol. Cell. Biol., 1991, 11, 2593-2608

\section{S03-09}

A Selection System for Somatic Recombinational Events in Drosophila melanogaster: Construction of the Test System and Initial Studies in Schneider Line 2 Cells.

Baertsch, S.; Sengstag, Ch. and Wuergler, F. Institute of Toxicology, Federal Institute of Technology of Zurich, CH-8603 Schwerzenbach. Genetic recombination is able to induce cancer and to mediate its further progression. In cells from cancer predisposed individuals, mitotic recombination can lead to loss of heterozygous tumor suppressor genes as is e.g. observed in the hereditary form of retinoblastoma. One mechanism for gene loss is inter- or intrachromosomal recombination involving short duplicated sequences. Our aim is to identify xenobiotics that are able to induce mitotic recombination and to elucidate the involved molecular mechanisms. For that purpose we have constructed transgenic $D$. melanogaster $(D . m$.). cell lines. Cells were stably transfected with an extrachromosomal DNA element providing a putative recombination substrate. The constructed element contained two truncated fragments of the neomycin resistance gene $\left(\mathrm{neo}^{\mathrm{R}}\right)$ interrupted by a hygromycin resistance marker (hyg ${ }^{R}$ ). The hyg ${ }^{R}$ insert was flanked on both sides by identical 352 bp fragments of neo ${ }^{R}$. Different $D$. $m$. promoters shown to be constitutively active in various $D . m$. tissues were inserted $5^{\prime}$ to both $E$. coll genes. Restoration of a functional neo ${ }^{R}$ gene may occur by deletion of the hy ${ }^{R}$ after recombination between the homologous regions. Stably transfected Schneider line 2 cells were obtained and preliminary results concerning chemical induction of genetic rearrangements will be shown.
S03-10

ANALYSIS OF MEIOSIS IN TOPOISOMERASE II DEFICIENT PISSION YEAST.

E. Hartsuiker, J. Bähler and J. Kohli.

Institute of General Microbiology, Baltzerstrasse 4. 3012 Bern, Switzerland.

We are analysing meiosis in a temperature sensitive top2 mutant. Complementary temperature-shift experiments showed that topoisomerase II is required for both meiotic divisions; early meiotic events are not impaired. In a meiotic time course, DAPI staining revealed that the cells are blocked before the first meiotic division. Interestingly, in meiotic spreads we found that the spindle pole body (SPB) cycle is not affected by the block: the final arrest point showed 1 nucleus with 4 completely separated SPBs. We want to analyse the number of SPBs and spindle formation with immunofluorescence. We are planning to analyse the course of meiosis in a top 2 rec7 double mutant (rec7 is recombination deficient) to see if topoisomerase II activity is necessary for separation of recombined chromosomes. Analysis of meiosis in a top2 rec8 double mutant (rec8 is recombination deficient and does not form linear elements) could give us a clue about the function of the linear elements.

\section{S03-11}

\section{CHARACTERIZATION OF MISMATCH-BINDING ACTIVITIES IN RROTEIN EXTRACTS OF SCHIZOSACCHAROMYCES POMBE}

Fleck, 0., Schär, P. and Kohli, J., Institute of General Microbiology, Baltzerstr. 4, 3012 Bern

To detect mismatch-binding proteins of $S$. pombe we performed band shift assays with radiolabeled oligonucleotides containing a defined mismatch. We found two distinct mismatch-binding activities. one shows high affinity to most of the mismatches, but is absent for $\mathrm{C} / \mathrm{C}$. This protein might belong to the general mismatch-repair system, which is thought to be related to the bacterial Muts dependent pathway. The second activity preferentially binds to those mismatches containing a cytosine and therefore represents a novel type of mismatch-binding protein.

\section{S03-12}

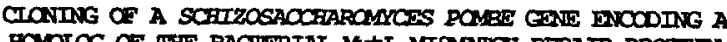
hOMOIOG OT THI BACIERTILL MUtI MISMATCH REPAIR PROTEIN

Baur M., Schär P. and Kohli J., Institute of General Microbiology, Baltzerstrasse 4, CH-3012 Bern

Homologs of the Salmonella typhimurium (mutL, $S$ and $U$ ) and Streptococcus pneumoniae (hexA, B) genes involved in mismatch repair have been identified in several distantly related organisms. So far no mismatch repair gene is known in Schizosaccharomyces pombe. In order to get more insight into the mechanisms of mismatch repair in $S$. pombe we started the cloning of a mut $I$ homolog. Degenerated oligonucleotide primers based on conserved regions of mut homologs were used in the polymerase chain reaction (PCR) to amplify a corresponding DNA fragment from S. pombe. A DNA fragment of about 220 bp was armolified whose deduced amino acid sequence shared a high degree of homology with PMSI of Saccharomyces cerevisiae and mutL, hexB. With the help of this DNA fragment the gene was isolated from a genomic DNA library by colony hybridization. Sequence analysis of this $\mathrm{mlhl}$ gene $(\mathrm{mlh}=$ mut $\mathrm{l}$ homolog) shows an $\mathrm{ORF}$ of the expected size with three putative introns. While the decuced amino acid sequence of the $5^{\prime}$ region shares strong homology with the procaryotic genes mut $L_{\text {, hexB }}$ and the eucaryotic gene PMS1, the amino acid sequence of the $3^{\prime}$ region shows homology only to PMSI and hexB. The central region of the protein seems to be conserved only weakly. 


\section{CHARACTERIZATION OF S.POMBE REC7 AND REC8 PROTRINS}

Parisi S,, and Kohli J., Institute of General Microbiology, University of Bern, CH-3012 Bern

Rec7 and rec8 are supposed to be genes with major functions in meiotic recombination since mutations in these genes reduce the frequency of meiotic intragenic recombination $\sim 1000$ fold. These genes have been isolated and sequenced but no enzymological activity could be assigned to the protein. A first step toward enzymological and biochemical characterization of rec7 and rec8 is to make both protein products available in sufficient quantities and in reasonable pure form. For this purpose both genes will be cloned in a $S$. pombe over-expression system using the strong and inducible nmt 1 promoter. In parallel both genes will also be expressed in E. coli as GST fusion proteins in order to produce polyclonal antibodies in rats and rabbits. These antibodies can then be used for protein purification in $S$. pombe as well as for immunofluorescence experiments. Induction of expression in $E$. coli produces a band of the expected size of $56 \mathrm{kDa}$ for Rec7 and of $73 \mathrm{kDa}$ for Rec8. To remove the GST carrier, the Rec7 and Rec8 fusion proteins can successfully be cleaved by thrombin or factor Xa respectively. Production of antibodies is in progress.

\section{S03-14}

Susceptibility to Scrapie Infection Correlates with Expression Level of Prion Protein PrpC

M. Fischer, A. Sailer, H. Büeler, T. Rülicke*, A. Aguzzi+, P. Autenried and C. Weissmann; Institut für Molekularbiologie I, Universität Zürich, Hönggerberg, 8093 Zürich, *Biologisches Zentrallabor and +Institut für Neuropathologie, Universitätsspital Zürich, 8091 Zürich, Switzerland

The infectious agent of transmissible spongiform encephalopathies such as Creutzfeldt-Jakob disease in man or scrapie in sheep is thought to be PrPSc, a postranslationally modified form of the normal host protein PrPC. We have shown that mice devoid of PrPC (Prn-polo) are completely resistant to scrapie. Here we report that also heterozygous Prn-pol+ mice with reduced levels of $\mathrm{PrPC}$ show enhanced resistance to scrapie, as manifested by a long delay in the onset and slow progression of clinical disease. Conversely, Prn-polo animals overexpressing PrP transgenes exhibit shortened incubation times and accelerated progression of the disease. Propagation of the scrapie agent is completely abolished in Prnpolo animals. High titers of infectivity, about $10^{8} \log$ LD50 units/g of brain, are detected in Prn-po/+ mice 33 weeks after inoculation (the earliest time point measured), 24 weeks or more before the animals die of scrapie. In contrast, wildtype animals survive no more than 16 weeks after reaching similar titers of infectivity. A practical implication of our findings is that moderate inhibition of $\mathrm{PrPC}$ synthesis could mitigate disease progression in spongiform encephalopathies.

\section{S03-15}

\section{ORGANISATION OF THE 3' END OF THE MOUSE B- APP GENE: POSSIBLE TARGET FOR ALZHEIMER'S DISEASE MODELS.}

Gschwind M. and Huber G., Pharma Division, Preclinical Research, F. Hoffmann-La Roche Ltd, CH-4002 Basel.

in some families with early onset Alzheimer's disease mutations on the B-APP gene have been identified which cosegregate with the disease. So far all these pathogenic mutations have been identified on exons 16 and 17 in humans framing the sequence of the B-amyloid itself.A genomic clone was isolated from mouse strain C57 Black/ 6 containing the coding regions of the last three exons (16-18) according to the human sequence. Sequencing of all three exons including the large $3^{\prime}$ non coding region showed a $100 \%$ homology to the previously described Balb/c mouse cDNA. Exon-intron boundaries were determined at the same positions as in human and rabbit genome and the flanking regions in the introns are also very similar. Not only is the homology between the human and mouse B-APP very high $(97.6 \%)$ but also the genomic organisation of their genes is nearly identical. Therefore homologous recombination can be applied to introduce molecular B-APP changes and to study their functional consequences on the protein level.
THE EFFECTS OF TRANSFECTED MUTANT AND WILD TYPE RAG GENES IN RECOMEINATION COMPETENT AND NON-COMPETENT CELLS

Döbbeling, U., Hobi, R., Berchtold, M.W. and Kuenzle, C.C. Institut für Veterinärbiochemie, Universität zürich, winterthurerstrasse $190, \mathrm{CH}-8057$ Zürich.

Normally, only pre B- and pre T-cells can rearrange their immunoglobulin genes by $V(D) J$ recombination, but when other cell types are transfected with the rag-1 and rag-2 genes they also become able to perform $V(D) J$ recombination. BY transient ly transfecting NIH 3T3 fibroblasts with both intact rag genes we found da recombination rate of 0.068 . No recombination was detected, when we transfected the intact rag-1 gene with a mutant rag-2 gene, which lacks $89 \mathrm{c}$-terminal amino acids, or the intact rag-2 gene with a rag-1 gene containing a deletion in the topoisomerase II homology region. These experiments show that these two regions of the rag genes are essential for $V(D) J$ recombination. When the L4 fibroblast cell line, which has been rendered recombination competent by stable transfection with the rag genes was overtransfected with both wild type rag genes, we found in contrary to an expected increase of $V(D) J$ recombination a reduction by a factor of 2-2.5. This decrease was not observed with the mutant rag genes. This result indicates, that there exists an optimal intraceliular RAG concentration for efficient $V(D) J$ recombination.

\section{S03-17}

\section{STRUCTURE AND FUNCTION OF RUVB PROTEIN.}

Stasiak. A., Bednar. J., Dubochet. J., Laboratoire d'Analyse Ultrastructurale, Université de Lausanne, CH-1015 Lausanne-Dorigny, Tsaneva, I.R., Dunderdale H., West. S.C., Imperial Cancer Research Fund, Clare Hall Laboratories, Herts., EN6 3LD, England, Egelman, E.H., Yu, X., Dept of Cell Biology and Neuroanatomy, University of Minnesota, Minneapolis, MN 55455, USA

The RuvB protein has been shown, biochemically and genetically, to be involved in the process of homologous recombination in $\mathrm{E}$. coli. One of its functions is to promote branch migration within Holliday junctions formed during the process of recombination. To investigate the mechanism by which RuvB promotes branch migration we have used conventional electronmicroscopy, cryo-electron microscopy, scanning transmission electronmicroscopy, and image analysis to study the interaction of RuvB protein with DNA. We observed that RuvB forms multimeric rings on dsDNA which encircle the DNA. We also observed that such rings tend to localize at the Holliday junctions. We propose that RuvB rings can actively move along DNA using the energy of ATP hydrolysis.This movement continues upon encountering a Holliday junction, thus forcing branch migration along the DNA.

\section{S03-18}

HOW RECA PROTEIN EXCHANGES STRANDS BETWEEN PARTIALLY HETEROLOGOUS DNA MOLECULES.

Andrzej Stasiak ${ }^{1}$, Patrice Morel $^{2}$, Dusko Ehrlich ${ }^{2}$ and Era Cassuto $^{2}$

${ }^{1}$ Laboratoire d'Analyse Ultrastructurale, Université de Lausanne, CH-1015 Lausanne-Dorigny, Switzerland.

2 Institut National de la Recherche Agronomique, 78352 Jouy en Josas, France.

We systematically investigated the ability of RecA protein to push the DNA strand exchange reaction through heterologous regions. We observed that RecA can push the strand exchange through substantial heterology (up to $150 \mathrm{bp}$ ) when the heterologous insert was placed on the so called proximal end of the linear duplex. In the case of the media heterology the strand exchange reaction could overcome heterologies of up to about $50 \mathrm{bp}$. Heterology at the distal end was most difficult for RecA to resolve, and already 22 bp long insert was completely blocking the progress of the strand exchange.

These results, together with earlier electron microscopy studies, allow to propose a molecular mechanism by which RecA can exchange strands between partially heterologous DNA molecules. 
S03-19

\section{RT-PCR TO STUDY HOMOLOGOUS RECOMBINATION IN BOVINE VIRAL DIARRHEA VIRUS}

Barbara Frech, Hanspeter Stalder and Ernst Peterhans institut für Veterinär-Virologie, Universität Bern, CH-3012 Bern

Bovine viral diarrhae virus is a positive-stranded RNA virus of the pestivirus group. Two biotypes, cytopathic and non-cytopathic in cell culture, can be distinguished. All cytopathic strains characterized so far carry insertions in their genomes. They probably result from a strand-switching activity of the viral RNA polymerase during viral replication. To study the switching capacity of the viral enzyme, RNA isolated from cells infected with two different strains of bovine viral diarrhea virus were analysed for homologous recombination in an RT-PCR assay. Performing the assay we found that fragmented RNA present during reverse transcription, the reverse transcriptase enzyme itself and viral RNA present in the PCR reaction result in the production of artificial recombination during the assay. It was not possible to detect a homologous recombination activity of the viral polymerase above the background recombination frequency of the RT-PCR assay. Our results strongly question the use of the RT-PCR assay for the study of recombination of RNA viruses.

\section{Viruses: The Paradigm for Symbiosis and Darwinian Evolution}

S04-01

MOLECULAR EVOLUTION IN E.COL: TRANSPOSITIONAL DNA REARRANGEMENTS OVER $10^{\prime} 000$ GENERATIONS

Papadopouios D. Blot M and Arber W.

Abt Mikrobiologie; Biozentrum der Universität; Klingelbergstr. 70 4056 Basel

Transpositional rearrangements mediated by insertion sequence (is) elements represent an important source of genetic variation within populations ${ }^{1}$. We analyze DNA rearrangements at the level of the complete genome by RFLP, using 7 is elements as probes for hybridisation to Southern blots. Samples taken at different time points from 12 independent cultures of E.coli B grown by serial transfer for $10^{\prime} 000$ generations ${ }^{2}$ and stored at $-70^{\circ} \mathrm{C}$ are used to study structure and evolution of genetic diversity within populations. The seven IS elements contribute differently to genetic variation and lead to a succession of mutants affecting the genetic structure of the population. We now search for correlations between RFLP patterns and the increase of relative fitness over time observed previously²

1 Naas T., Blot M. Fitch W.M. and Arber W. (1993) Genelics: in press

2 Lenski R.E. Rose M.R., Simpson S.C. and Tadler, S C (1991) Am. Nat.138, pp 1315. 1341

\section{S04-02}

THE DNA SEQUENCE OF THE L.MIGRATORIA MITOCHONDRIAI GENOME

P.K.Flook, C.H.F. Rowell \&.Gellissen*

Zoologisches Institut, Universitat Basel

${ }^{*}$ Rhein Biotech GmbH, Dusseldorf

The complete DNA sequence of the Locusta migratoria mitochondrial genome has been derived from four cloned mtDNA fragments. The sequence is $15.2 \mathrm{~kb}$ in length, contains 13 peptide coding sequences, and genes for 22 tRNAs and two rRNAs. The organisation of the genome shows a strong resemblance to Drosophila, the gene order and orientation being the same except for the positions two tRNAs. This contrasts with the only other non-dipteran insect mtDNA to have been sequenced, Apis mellifera, which shows differences in the positions of 11 tRNAs. This finding is discussed in relation to the AT content of the different genomes. The sequences of the three mtDNA genomes are also compared directly and the results related to existing knowledge concerning the evolution of insects.

\section{S04-03}

\section{REPLICATING ARTIFICIAL SUBGENOMIC MEASLES RNA}

K. Kaelin, P. Spielhofer, F. Radecke, H. Schneider, C. Kunz, M.A. Billeter, Molekularbiologie I, Universität Zürich, Hönggerberg M.S. Sidhu, P. Dowling, S.A. Udem, New Jersey Medical School, Newark, NJ 07103-2714, US A

Nonsegmented negative strand RNA viruses are so far not amenable to reverse genetics. The genomes must associate with nucleocapsid $(N)$, phosphoprotein and polymerase proteins to form a functional RNP. As a step towards reverse genetics of measles virus (MV), plasmid vectors were constructed allowing synthesis of RNAs corresponding precisely to a MV genome in which all coding and intercistronic regions are replaced by the chloram-phenicol acetyl transferase (CAT) coding region. Transfection of these negative polarity transcripts into MV infected HeLa or 293 cell lines gave rise to CAT activity which could be serially transferred and massively amplified together with progeny helper virus in cell cultures. Both expected MV/CAT chimeric mRNA and replication product were identified in the progeny. Preliminary experiments indicate that only RNAs in which the total number of nucleotides is a multiple of six replicate and transcribe efficiently, consistent with the data on natural and modified copyback DI RNA of Sendai virus (Calain and Roux, J. Virol. 674822 (1993)). Precise end to end encapsidation of RNA, each $\bar{N}$ protein contacting six nucleotides, might be required.

\section{S04-04}

TRANSGENIC MICE PROVIDE TISSUE-SPECIFICITY MODEL FOR HUMAN CONGENTTAL CYTOMEGALOVIRUS INFECTION.

\author{
M. Koedood, P. Meier, A. Fichtel and P.J. Mitchell \\ Inst. f. Molekularbiologie II, Universität Zürich \\ Winterthurerstr. 190, 8057 Zürich, Switzerland
}

The embryonic tissue specificity of the human cytomegalovirus immediate-early (hCMV-IE) promoter/enhancer $(-522$ to +13$)$ was examined by $\beta$-galactosidase staining of transgenic mouse embryos carrying hCMV-IE regulated lac $Z$ genes. Embryos of three transgenic lines were analyzed between $8.5-13.5 \mathrm{dpc}$. For all lines, hCMV-IE transcription was primarily confined to subsets of neural and vascular cells. However, extents of transgene expression along the embryonic primary axis were slightly and greatly restricted in two of the lines relative to the third line at all stages analyzed. These differences most likely represent integration site-dependent chromatin constraints that affect levels of hCMV-IE-directed transcription. Together, the three lines can be taken to define a graded potential for hCMV-IE transcription along the anteriorposterior axis of the embryo with greatest permissivity in mid-axial structures which include the dorsal neural tube at the level of the hindbrain and upper cervical spinal cord, the inner ear and vestibular acoustic ganglia, branchial arteries, aortic sac, and lung buds. The cell typespecificity and axially graded permissivity for hCMV-IE transcription in the mouse embryo provide a basis for understanding the pathology and relative frequencies of different types of birth defects caused by congenital hCMV infection in humans. 
S04-05

EXPRESSION OF HUMAN PAPIIIOMAVIROS TYPE 18 (HPV18) CAPSID PROTEINS IN E. COLI AND MAMMAIIAN CELLS.

Stauffer, $Y$.. Offord, $E$. and Beard, P., Swiss Institute for Experimental Cancer Research (ISREC), CH-1066 Epalinges, Switzerland.

HPV1 8 is associated with genital carcinoma. Like other human papillomaviruses, HPV18 cannot be easily propagated in cell culture, because the viral growth cycle is dependent on the differentiation of its host cell, the keratinocyte. Moreover, very little viral capsid protein is found in vivo in infected tissues. The HPV18 L1 and I2 genes code for the major and minor capsid proteins, respectively. To obtain the viral capsid proteins we made constructs with the II and L2 genes for expression in E. coll (the pQF system) and in recombinant vaccinia virus (a modification of the pHGS-1 system) infected (a modification of the pHGS-1 system) infected contained a histidine tag encoded by the vector. This allowed us to purify the proteins on a nickelaffinity column in order to raise antibodies. These will permit us to detect the presence of capsid proteins expressed from recombinant vaccinia viruses. We expect the LI and L2 proteins expressed together from the vaccinia-based vector to selfassemble to form virus-like particles.

\section{S04-06}

PAPAIN-LIKE VIRAI, PROTEINASES

Kadaré G., Rozanov M. and Haenni A. - I.

Institut Jacques Monod, 2 place Jussieu, 75251 paris Cedex 05, France.

Most viral proteinases are either chymotrypsin-like or papain-like enzymes. The sequence of viral papain-like proteinases contains two weakly conserved motifs with essential Cys and His residues. Remote similarities with these motifs have been found in certain viral (tymoviruses, alphaviruses, etc.) and cellular proteinases.

The polyproteir of turnip yellow mosaic tymovirus (TYMV) undergoes autocleavage yielding products of $\sim 150 \mathrm{~K}$ and $\sim 78 \mathrm{~K}$. The viral proteinase responsible for this cleavage has been investigated. Potential catalytic Cys783 and His869 residues of the TYMV polyprotein have been identified in a stretch of sequence distantly related to papain-like proteinases. The role of these putative catalytic residues in the activity of the proteinase was studied by site-directed mutagenesis. A minimal proteinase has also been constructed by $\mathrm{N}$ - and C-terminal deletion to determine the boundaries of the proteolytic domain. Sequence analysis of the c-terminal cleavage product should indicate the site of cleavage.

\section{S04-07}

REGULATION OF GENE EXPRESSION IN BACTERIOPHAGE P1, A PROTOTYPE FOR MICROBIAL SYMBIOSIS AND EVOLUTION $\mathrm{Ch}$. Schmidt and W. Arber; Biozentrum der Universität Basel, Abt. Mikrobiologie, Klingelbergstr. 70, $\mathrm{CH}-4056$ Basel

Bacteriophage $\mathrm{P} 1$, carried as a single copy plasmid in $E$. coll, is widely known for its horizontal gene transfer ability (transduction) in Enterobacteria. As a temperale phage, it can lysogenize its host and thereby provides it with accessory functions (restriction of foreign DNA by EcoP1, functions expressed by transposons carried on the P1 genome or integrative suppression). These properties reflect both symbiotic and evolutionary functions of $\mathrm{P} 1$.

P1 regulates expression from its genes by two different mechanisms: transcription of early regulatory genes is repressed by the phage encoded repressors $\mathrm{C} 1$ and Bof, whereas transcription of its morphogenetic genes from P1 specific late promoters is induced by the early expressed gp10. By site-directed mutagenesis the late promoter PS, composed of the -22 inverted repeat AAGTTACTT and the -10 box, was functionally characterized. $P_{S}$ and its derivatives compare well with several other late promoters of P1 with regard to their sequence dependent strengths: the strong promoters PS, $P_{\text {dar }}$ and $L P r 2 b$ all share the perfect inverted repeat around position -22 , whereas the weaker promoters LPr91, LPr82a, LPr82b and LPr83 contain mismatches in their inverted repeats or the inverted repeat is positioned more upstream relative to the transcription initiation site.
S04-08

TENUIVIRUSES - A NOVET GROUP OF PLANT VIRUSES - AND THEIR RELATIONSHIP TO NEGATIVE-STRAND RNA VIRUSES Eamírez,B._c., Garcin, D. ' Kolakofsky, D. , \& Haenni, A. - L * Univ. of Geneva Medical School, 1205 Geneva, Switzerland.

Five viruses belong to the recently recognized Tenuivirus group, rice stripe virus (RSV), maize stripe virus (MStV), rice hoja blanca virus (RHBV), European wheat striate mosaic virus, and rice grassy stunt virus (RGSV). These viruses infect plants of the Graminae family. They are persistently transmitted by planthoppers in which they are transovarially passed. The "viral particles" isolated from Tenuivirus-infected plants have a flexuous, circular thread-like morphology. Their genome is composed of four (RSV, RHBV, RGSV) or five (MStV) single-stranded RNA. The cDNA sequences of the genome segments so far determined suggest that the genome of Tenuiviruses is mainly of ambisense nature. The ambisense strategy is also used by the Arenaviridae and some members of the Bunyaviridae. The RHBV mRNA are synthesized via a cap-snatching mechanism similar to the one described for the synthesis of the mRNA of influenza virus, and of some members of the Arenaviridae and Bunyaviridae families.

\section{S04-09}

CELI ENTRY, UWCOATING AND NUCLEAR TRANSPORT OF ADRNOVIRUS 2 .

U. F. Greber and A. Helenius.

Yale University School of Medicine, New Haven, CT, U.S.A.

To enter cells, viruses raust achieve three major goals: transfer their genone across the plasma membrane into the cytosol, target the genome to the replication site and decondense it for replication. Adenovirus, an icosahedral nonenveloped particle with a double-stranded genomic DNa and 11 to 15 structural proteins, enters into human $\mathrm{KB}$ cells by a co 15 structural proteins, enters into human $\mathrm{kB}$ cells by endocytosis, acid-dependent penetration into the cytosol, and endocytosis, acid-dependent penetration into the cytosol, and targeting to the nucleus. During intermalization, the tibers,
primarily responsibie for virus binding to the cell surface, are detached. In early endosomes, the capsid-stabilizing proteins IIIa and VIII are released. Fenton base, a vertex associated protein implicated in penetration across the endasomal membrane, is removed in endosones, if penetration is blocked with lysosomotropic reagents. In the absence of inhibitors, a large fraction of penton base stays associated with the cytoplasmic capsid. The viral DNA is disconnected from the shell after proteolysis of the internal protein VI, a capsid and DNA binding component. Removal of protein IX, a capsid binding factor, further destabilizes the coat, and the remaining 'stripped-down' virus particles associate with nuclear pore complexes to release their DNA-genores into the nuclear pore complexes to release their DNA-genomes into the provide the high efficiencies of the entry process needed to provide the high efficiencies of the entry process needed to successfully deliver the viral DNA across
the cell nucleus, the site of replication.

S04-10

CLINICAL ISOLATES OF ACTINOBACILLUS ACTINOMYCETEMCOMITANS (AA) FROM THREE EUROPEAN CITIES CARRY HIGHLY RELATED PROPHAGES

K. WILII, H. SANDMEIER, J. MEYER

Dept. of Preventive Dentistry and Oral Microbiology, Dental Institute, university of Basel

Dental plaque bacteria are the cause of periodontal diseases. One of the putative periodontal pathogens is the Gram-negative, facultatively anaerobic $\mathrm{Aa}$. Earlier work had suggested that bacteriophages of Aa might be associated with disease activity. Five lysogenic Aa strains isolated from patients from three geographic locations (Basel, Freiburg i.Br., Ansterdam) belonged to two genotypes determined by RFLP analysis. The tenperate bacteriophages induced from these hosts were related: Electron microscopy revealed for all phages an isometric icosahedral head a contractile tail with a base plate and up to four fibers. All five phages had a genome size of about $43 \mathrm{~kb}$ and showed identical or similar restriction fragment patterns. Hybridization experiments indicated extensive DNA homologies. However, the phages formed two immunity groups. The results may suggest a common genetic trait providing a selective advantage to lysogenic $\mathrm{Aa}$. 
S04-11

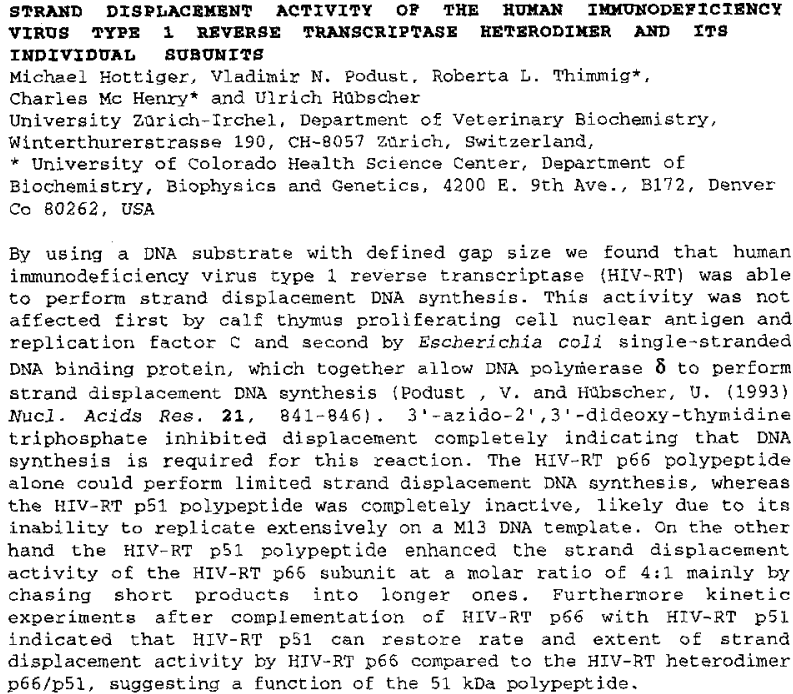

S04-14

EXPRESSION OF THE MOUSE MAMMARY TUMOR VIRUS ORF PROTEIN IN MAMMALIAN CELLS USING RECOMBINANT VACCINIA VIRUSES.

C. Krummenacher and H. Diggeimann

Institute for Microbiology, University of Lausanne, Ch. des Boveresses 155 $\mathrm{CH}-1066$ Epalinges, Switzerland

The mouse mammary tumor virus proviral DNA contains an open reading frame in the $3^{\prime}$ long terminal repeat which can code for a $36 \mathrm{kDa}$ polypeptide with a putative transmembrane sequence and five $\mathrm{N}$-linked glycosylation sites. This gene is known to code for a superantigen which deletes a specific subset of $\mathrm{CD}^{+}{ }^{+} \mathrm{T}$-lymphocytes in vivo. The superantigen encoded by the exogenous mouse mammary tumor virus of the GR strain acts specifically on VB14 bearing T-cells.

We produced recombinant vaccinia viruses to express either the complete or a truncated ORF protein after infection of primate cells in culture. The complete orf gene in mammalian cells leads to the production of a $47 \mathrm{kDa}$ protein which is specifically detected with an anti-ORF-peptide antiserum. The $47 \mathrm{kDa}$ protein can be labeled with $\mathrm{D}-[2-3 \mathrm{H}]$ mannose and its synthesis is inhibited by tunicamycin, an $\mathrm{N}$-linked glycosylation inhibitor, indicating that it is a glycoprotein. The truncated ORF protein beginning at the second ATG of the open reading frame is aiso modified, but the C-terminal half of ORF, starting at the fifth ATG (ORF5), has the expected size of the non modified polypeptide. Pulse-chase experiments indicale that the $47 \mathrm{kDa}$ ORF glycoprofein has a short half-life of about 1.5-2 hours in this system whereas the ORF5 truncated protein is even less stable; its half-life is about 20 minutes.

The cellular localization of the ORF protein and the role it could play in vivo are currently under investigation.

\section{S04-15}

\section{EVOLUTION OF MEASLES VIRUS IN BRAIN DISEASES}

R. Cattaneo, P. Spielhofer, K. Kaelin, M.A. Billeter

Molekularbiologie I, Universität Zürich, Hönggerberg, 8093 Zürich

Subacute sclerosing panencephalitis (SSPE), a rare, letha disease is caused by clonally selected defective measles viruses (dMVs) characteristically mutated. This emerged from sequencing of five MV genes from SSPE cases and by functional tests of the envelope glycoproteins hemagglutinin $(H)$ and fusion protein $(F)$. These expressed $H$ and $F$ variants migrate poorly to the cell surface but maintain some cell fusion activity whereas the envelope associated matrix protein $(\mathrm{M})$ appears dispensable. Thus, the spread of dMV genomes through the brain seems to occur by membrane fusion at local cellular contacts, essentially hidden from the massive immune responses.

In more than half of SSPE cases, the propagated dMVs contain $\mathrm{M}$ genes in which up to $50 \%$ of $U$ residues are replaced by $\mathrm{Cs}$. Such "biased hypermutations" are likely due to the simultaneous conversion of many A residues to inosine in double stranded RNA regions accidentally formed, mediated by an ubiquitously occurring adenosine deaminase. Similar events at less spectacular degrees have now been observed in functional genes of several negative strand RNA viruses. Thus, this enzyme might be an important evolutionary driving force for Mononegavirales.

\section{S04-16}

\section{FATAL LIVER FAILURE AFTER TRANSPLANTATION OF A HEART FROM A HEPATITIS B VIRUS (HBV) POSITIVE DONOR: EMERGENCE OF HBV VARIANTS WITH A DELETION IN THE PRE-S 1 GENE}

S04-13

\section{DIFFERENTIAL CELL SUSCEPTIBILITY TO THE MOUSE MAMMARY} TUMOR VIRUS

F. Luthi, K. Erny and H. Diggelmann

Institute for Microbiology, University of Lausanne, Ch. des boveresses 155 , Ch-1066 Epalinges, Switzerland

Mouse mammary tumor virus (MMTV) is produced in the mammary gland of infected females and transmitted by the milk to suckling new-borns. The passage of MMTV from the gut to the mammary gland is still poorly understood, the nature of the tissue tropism of MMTV has not been elucidated. A single receptor present on mammary epithelial and lymphoid cells might serve for the entry of the virus. A cell-type specific entry mechanism could in part be responsible for this phenomenon. Alternatively different surface molecules might be involved in its uptake.

We have studied the cell susceptibility to MMTV using a sensitive method of detection of newly acquired exogenous proviral DNA sequences, prior to viral integration and transcription. Thereby we confirm that MMTV infects mouse, rat and monkey cell lines of different tissue origin in vitro demonstrating the lack of a strict host range specificity in tissue culture as previously described. We have also observed the infection by MMTV of various human cell lines. Furthermore various infection susceptibilities are observed for cell lines of a same species.

\section{Pult, I., Wieland, S., Niederöst, B., Keist, R., Walter, E., and Blum, H.E.} Department of Medicine, University Hospital Zürich

Infection by HBV-positive organ donors remains a serious problem in transplant surgery. Here we present a case of a 58-year-old woman who received a heart transplant from a hepatitis $\mathbf{B}$ surface antigen (HBsAg) positive donor. The patient was negative for all HBV markers prior to transplantation. Two months after transplantation she became HBsAg positive and died from subacute liver failure about seven months later.

Cloning and sequencing of HBV DNA revealed several HBV mutants in the serum of the recipient, each with a $108 \mathrm{bp}$ in-frame deletion in the pre-S 1 region. Transfection studies in HuH-7 cells with the predominant pre-S I deletion mutant showed a higher replication competence compared to wild-type HBV. Sequence analysis of PCR products from serum of the donor showed mainly wild-type HBV DNA in the pre-S region without the pre-S 1 deletion.

These data demonstrate that mutant viruses accumulate in immunosuppressed patients. Specific mutants may play a critical role in the severe or fatal clinical course of $\mathrm{HBV}$-infection in transplanted patients. 
S04-17

INTERACTION OF NEURONAL OCT FACTORS WTTH HERPES SIMPLEX VIRUS IMMEDIATE EARLY GENE PROMOTERS

Philippe Douville, Michael Hagmann, Oleg Georgiev and Walter Schaffner

Institut für Molekularbiologie II, der Universität Zürich, Winterthurerstr. 1908057 Zürich

In non-neuronal cells Herpes Simplex Virus (HSV) establishes a productive lytic infection starting with transcription of its immediate early (IE) genes by corecruitment of a viral protein (VP16) and a cellular factor (Oct-1) onto characteristic octamer-like sequence motifs (TAATGARAT) in the promotors of the IE genes. In neurons, however, HSV establishes latent infections whereby transcription of the IE genes is prevented and the lytic program aborted. Our efforts are focused towards determining which neuronal factors are involved in the regulation of HSV IE gene transcription and whether they may be involved in HSV latency or reactivation in neurons. In electrophoretic mobility shift assays (EMSA) using mouse brain nuclear extracts we found that neuronal Oct factors (NOct-2, NOct-3 and NOct-4) bind to TAATGARAT sequences from the viral ICP0 and ICP4 promoters. An additional brain protein also binds to these sequences but not to a consensus octamer sequence (ATGCAAAT) as do neuronal Oct factors. EMSA assays with mutated TAATGARAT probes show that the TAAT sequence is crucial for this binding and we have tentatively named this brain protein TAAT-1. We are currently testing the NOct factors in in vivo and in vitro transcription assays to study their involvement in HSV IE promoter regulation.

\section{S04-18}

BIOCHEMTCAL AND FUNCTIONAL ANALYSIS OF A VACCINIA VIRUS RECOMBINANT CONTAINING TWO COPIES OF THE P37K GENE

Schmutz, C., and Wittek, R.

Institut de Biologie Animale, Université de Lausanne, CH-1015 Lausanne.

The most abundant protein of the vaccinia virus envelope is the palmitated $37 \mathrm{~K}$ protein. This protein is required for envelopment and subsequent release of virus from infected cells. The amount of extracellular enveloped virus (EEV) produced varies considerably depending on the virus strain and host cell line but remains in any case low ( $<30 \%$ of the virus production). If $\mathrm{P} 37 \mathrm{~K}$ is a limiting factor for envelopment, its overexpression might be a means to increase the production of EEV. For this purpose a vaccinia virus recombinant containing a second copy of the P37K gene was engineered. Western blot analysis clearly showed increased levels of this protein. Titration assays revealed slightly lower yields of both intracellular naked virus (INV) and extracellular enveloped virus (EEV) in cells infected with recombinant virus. Unexpectedly, with recombinant virus, the ratio of EEV versus INV was significantly lower when compared to wild-type virus infection, despite the higher level of P37K expression. We are currently testing whether this is a consequence of a lower production, or a reduced infectivity of EEV particles in cells infected with recombinant virus.

\section{S04-19}

\section{EVOLUTIONARY STASIS OF BOVINE VIRAL DIARRHEA VIRUS IN IMMUNOTOLERANT ANIMALS}

Hanspeter Stalder, Christian Hertig and Ernst Peterhans

Institut für Veterinär-Virologie, Universität Bern, $\mathrm{CH}-3012$ Bern

Bovine viral diarrhoea virus (BVDV) causes

acute transient infection in animals exposed to virus postnataly and persistent infection in animals infected in utero in the early phase of gestation. The latter is associated with specific immunotolerance to the infecting viral strain. Animals infected in utero may be born normal and remain healthy despite the persisting infection with a noncytopathic biotype of BVDV. Mutation to the cytopathic biotype, or superinfection with an antigenically similar cytopathic biotype, results in lethal mucosal disease. We have $\mathrm{PCR}$ amplified and sequenced parts of the region coding for the surface glycoprotein E2 and p80, a nonstructural protein associated with enzymatic activities. Over a time of one year, virus obtained from an immunotolerant persistently infected animal remained identical in its nucleotide sequence in the analyzed regions. Contrasting with this remarkable evolutionary stasis over some $600-800$ virus generations is the heterogeneity of viral isolates obtained from different persistently infected animals. We propose that the multilineage distribution of BVDV is the result of antigenic drift in the population of acutely infected animals, combined with evolutionary stasis in immunotolerant animals.
S04-20

Mapping of the Immunodominant Epitopes of the Caprine ArthritisEncephalitis virus gp 38 Transmembrane Protein

G. Bertoni*, M-L. Zahno*, H-R. Vogt*, R. Zanoni*, G. RuffS, W. P. Cheevers*, P. Sonigo ${ }^{+}$, E. Peterhans ${ }^{*}$ and G. Pancino ${ }^{+}$

* inst. of Vet. Virol. and SInst. of Animal Breeding, Uni. Bern

-Depart. of Vet. Microbiol. and Pathol., Washington State University, USA

+ Institut Cochin de Génetique Moléculaire (CNRS UPR0415), Paris, France

Caprine arthritis-encephalitis virus (CAEV) is a lentivirus causing clinical arthritis in 20 to $30 \%$ of naturally infected goats. The severity of disease in infected animals correlates with the antibody titers to the viral envelope protein (env) and especially to the gp 38 transmembrane portion (TM). In order to analyze linear $B$ epitopes of env, we produced a random library of CAEV env polypeptides fused to $\beta$-galactosidase and expressed in $E$. coli. The complete env gene obtained from an infectious clone of CAEV-CO was subcloned in a pUC18 plasmid and DNAse digested. Random fragments of 200 bp were inserted in $\lambda g t 11$ DNA and expressed in E. coli Y1090. As the first step of B epitope mapping and in order to identify immunodominant group epitopes we used high titer sera from naturally infected Swiss goats to screen the library. Six immunoreactive clones were obtained and subsequently sequenced. All six mapped to the TM region of env. A fine mapping of these immunoreactive regions was performed using pepscan technology and ELISA with synthetic peptides. Four epitopes could be identified including the cysteine loop corresponding to an immunodominant epitope in other lentiviruses. These peptides were used in vitro to test the reactivity of sera from naturally and experimentally CAEV-infected goats and in vivo to modulate the immune response of goats to TM.

S04-21

HORIZONTAL, GENE TRANSFER IN THE EVOLUTION OF BACTERIOPHAGE TAIL FIBER GENES

HEINRICH SANDMEIER

Abt. Präventivzahnmedizin und orale Mikrobiologie, Zahnărztliches Institut, Universität Basel Molecular analysis testifies of a surprising sharing of short gene segments among a variety of large dsDNA bacteriophages of enteric bacteria. Genomes from otherwise unrelated phages including lambda, Mu, P1, P2 and T4 must have exchanged parts of their tail fiber genes. Individual genes appear as mosaics with parts taken from a common gene pool. Therefore, horizontal gene transfer emerges as a major factor in the evolution of a specific part of phage genomes. Current concepts of homologous recombination cannot account for the formation of such chimeric genes, and the responsible recombinational mechanisms are not known. However, recombination sites for DNA invertases and recombination site-like sequences are present at boundaries of gene segments conferring host receptor specificity. This and the properties of the DNA inversion mechanism suggest that these site-specific recombination enzymes could be responsible for the exchange of host range determinants.

$504-22$

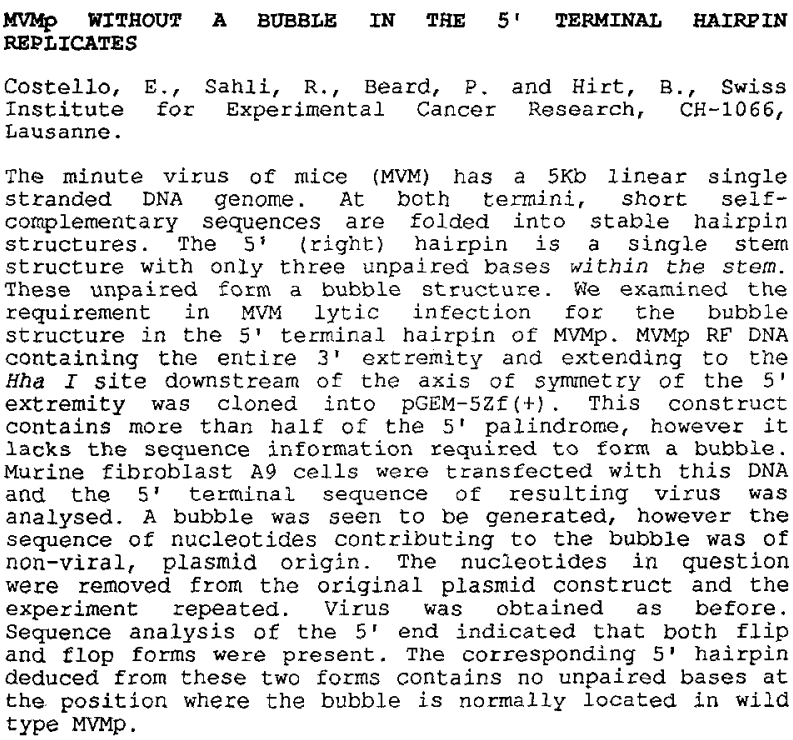
tha $I$ site downstream of the axis of symmetry of the 5 extremity was cloned into pGEM-5zf(t). This construct contains more than half of the $5^{\prime}$ palindrome, however it lacks the sequence information required to form a bubble. Murine fibroblast $A 9$ cells were transfected with this DNA and the $5^{\prime}$ terminal sequence of resulting virus was analysed. A bubble was seen to be generated, however the sequence of nucleotides contributing to the bubble was of sequence of nucleotices cont ributing to the bubble was of non-viral, plasmid origin. The nucleotides in question were removed from the origlnal plasmid construct and the experiment repeated. Virus was obtained as before. sequence analysis of the $5^{\prime}$ end indicated that both flip and flop forms were present. The corresponding 5' hairpin deduced from these two forms contains no unpaired bases at the position where the bubble is normally located in wild type MVMp. 
SO4-23

TRANSCRIPTIONAL ACTIVATION BY THE HERPES SIMPLEX VIRUS PROTEIN VP16 IN VITRO

Michael Hagmann, Philippe Douville, Oleg Gueorguiev, Walter Schaffner Institut für Molekularbiologie II der Universität Zürich, CH 8057 Zürich

The immediate early (IE) gene promoters of herpes simplex virus type-1 share a similar sequence which in some cases overlaps with a binding site for octamer binding proteins. This sequence confers responsiveness to a viral transactivator, VP16. On these promoters, VP16 combines with multiple cellular proteins to form an activating complex. We set up a reconstituted in vitro system consisting of HeLa nuclear extract and recombinant Oct-1/POU domain protein (without the activation domain of Oct-1) and VP16 where we showed that the activation of IE genes is not mediated by Oct-1 itself. Rather, Oct-1 serves to recruit VP16 that has no intrinsic DNA binding capacity. VP16, however, possesses a stong acidic $C$ terminal activation domain of $\sim 80$ amino acids which is indispensable for the in vivo function of the protein. As this and other acidic activation domains possess only a relatively weak activating capability when we test them in in vitro transcription assays we are interested in investigating the role of this acidic activation domain in vitro i.e. under condition of cell free extracts and on naked template DNA. We are using recombinant Oct1/POU domain protein and recombinant VP16 that has been truncated at the $\mathrm{C}$ terminus. Additionally, we are also testing different truncations of VP16 in vitro that have been produced in cos cells. Furthermore, we are also trying to see whether these different truncations already affect the assembly of the actvating multiprotein complex.

\section{S04-24}

\section{EXPRESSION OF CYTOKINE GENES IN ARTHRITIC JOINTS OF GOATS INFECTED WITH CAPRINE ARTHRITIS ENCEPHALITIS VIRUS}

F. Lechner\$, P. Peveri \$, A. Zurbriggen\# and E. Peterhans\$, Institutes of Veterinary Virology $\$$ and Animal Neurology ${ }^{\#}$, University of Berne, Switzerland

Caprine arthritis encephalitis (CAE) virus is a lentivirus that causes persistent infection in goats. Main symptoms of CAE include arthritis, mastitis and pneumonia. In addition, in some animals weight loss is observed and young animals may develop encephalitis. Histologically, the lesions caused by CAE virus are characterized by mononuclear inflammation but the mechanisms maintaining the inflammation over several years are poorly understood. To address this question, we are analyzing, by in situ hybridization using digoxigenin-labelled riboprobes, the expression of the viral genome relative to that of an array of cytokines including TNF- $\alpha$; IFN- $\gamma$, IL- $1 \beta$, IL-2, IL-8 and GMCSF. Initial experiments suggest that viral genome expression is restricted in the inflammatory infiltrates whereas certain cytokines, particularly TNF- $\alpha$, are strongly expressed in the synovial membranes. Overall, the results point to a prominent role of macrophage activation in the maintenance of chronic inflammation and possibly also in the development of arthritic lesions.

$504-25$

\section{Sequence differences between a cell adapted virus and the wild type (wt) virus of Porcine epidemic diarrhoea vírus (PEDV) strain CV777}

Tobler, K. and Ackermann, $M$.

Institut für Virologie, Winterthurerstrasse 266a, 8057 Zürich,

Coronaviruses include a group of RNA viruses of medical and veterinary interest. Porcine epidemic diarrhoea virus, a member of this viral group, causes enteritis in pigs.

The sequence of two cell culture adapted strains of PEDV has been reported previously. The RNA used for cloning and sequence determination has been extracted from Vero cells infected with PEDV strains CV777 or Br1/87. Differences between the strains were seen almost exclusively in a region termed ORF3, located between the $S$ and $M$ genes. Three populations of CDNA clones with different open reading frames were detected.

In contrast, upon sequencing of the wt virus obtained from pigs infected with the original isolate these differences have not been observed. We currently investigate for possible biological functions of the putative proteins encoded by the variable ORFs using polycional antibodies and transient expression assays.
S04-26

RFACTIVATION OF TRANSCRIPTION EROM A VACCINIA VIRUS EARLY PROMOTER LATE IN INEECTION

Masternak, K., wittek,R. Institut de biologie animale, Université de Lausanne, Switzerland

We have studied the kinetics of synthesis of transcripts initiated from the vaccinia virus $7.5 \mathrm{kD}$ gene early promoter. Unexpectediy, after a first burst of RNA synthesis early in infection, the promoter showed a second peak of activity in the late phase. Reactivation of transcription was neither dependent on the presence of the sequences upstream of the early promoter, nor on the location of the promoter in the genome. Reactivation seems to be dependent on virus assembly since it is prevented by rifampicin, that specifically inhibits an early step of viral morphogenesis. Analysis of several other early genes showed that reactivation is not unique to the $7.5 \mathrm{kD}$ early promoter.

\$04-27

Expression of the Mouse Mammary Tumor Virus env proteins with a recombinant vaccinia virus expression system.

R. Fuchs and $H$. Diggelmann, Institute of Medical Microbiology, University of Lausanne, Switzerland.

A recombinant vaccinia virus has been made with the MMTV env gene. The foreign gene is expressed under the influence of the vaccinia late promoter, $11 \mathrm{~K}$. In CV1 and COMMA cells infected with the recombinant virus, expression of the precursor Pr73 env and of the processed glycoprotein gp52 has been demonstated by immunoprecipitation of 35S-methionine labelled proteins with a polyclonal antigp52 antibody. 3H-mannose labelling followed by immunoprecipitation demonstrated that the precursor and the gp52 are glycosylated. Western blotting was also used to demonstrate synthesis of the precursor and gp52 glycoprotein. Immunofluorescence with a monocional anti-gp52 lgA antibody on permeabilised unpermeabilised celis demonstrated the presence of gp52 in the cytoplasm and at the cell surface of infected $\mathrm{CV} 1$ and COMMA cells. In vivo immunization assays in mice show an MMTV specific humoral response to subcuteanous adrninistration of the recombinant vaccinia virus at the tail base. Further characterization of the immune response is underway.

S04-28

\section{SEPARATELY EXPRESSED DOMAINS OF THE DUCK HEPATITIS B VIRUS CORE PROTEIN CAN FORM REPLICATION COMPETENT CORE-PARTICLES IN VITRO. \\ Wieland, S., von Weizsäcker, F, and Blum, H.E. Department of Medicine, University Hospital Zürich.}

The core protein of the hepatitis B virus (HBV) interacts with the viral RNA pregenome and the reverse-transcriptase/DNA-polymerase to form a core particle which allows for replication of the viral genome. Deletion analysis of the core protein revealed, that the Arg-rich carboxyterminal domain is required for RNA encapsidation and productive viral DNA synthesis.

We demonstrate, that the duck hepatitis B virus (DHBV) core protein can be devided into two domains comprising amino acids 1-67 (N-ter) and 67-262 (C-ter), respectively. The co-expression of these two core subdomains leads to the formation of replication competent core particles. Furthermore, we demonstrate that the carboxy-terminal domain of DHBV core (C-ter) can be functionally replaced by the corresponding domain of the human HBV. The complete HBV core protein, however, does not form replication competent core particles with the DHBV pregenome and RT.

These results imply that the carboxy-terminal part of the HBV core proteins define an exchangeable, functionally conserved domain. 
ACTIVATOR TRAP - CLONING OF TRANSCRIPTIONAL ACTIVATION DOMAINS BY EXPRESSION SELECTION IN MAMMALIAN CELLS

Matthias Gstaiger, Thomas Stamminger ${ }^{*}$ and Walter Schaffner Institut für Molekularbiologie II der Universität Zürich, CH 8057 Zürich

*Institut für Klinische Virologie, Universität Erlangen

Enhancer type transcriptional activation domains have been found to have a high content in acidic or serine/threonine amino acids. Since the number of characterized enhancer type activation domains is limited, more examples are needed to define a general structural motif for such activation domains. Thus we developed a novel technique (activator trap) for cloning transcriptional activation domains. Sonicated fragments of the DNA to be analyzed for the presence of an activation domain are fused to the GALA binding domain (aa 1-93) in an expression vector containing the SV40 origin of replication. Through trans- dependent replication selection in monkey cells we have re-isolated the activation domains of the VP16 protein from herpes simplex virus and the immediate early protein (PIEP) from pseudorabies virus. In addition, an as a yet uncharacterized activation domain from the infected cell protein 4 of bovine herpesvirus 1 (BICP4) and from a hitherto non-characterized ORF within the cytomegalovirus (CMV) genome have been isolated. All of these domains are active from a remote enhancer position. The activation domains of VP16, PIEP and BICP4 have a high content of negatively charged amino acids whereas the CMV ORF seems to belong to a novel class of activation domains.

\section{S04-30}

\section{EVOLUTION AND PATHOGENICITY OF LENTIVIRUSES.}

Pierre Sonigo. Génétique des virus (CNRS UPR 0415), Institut Cochin de Génétique Moléculaire, 22 rue Méchain, 75014 Paris, France

Lentiviruses define a subfamily of retroviruses. They cause persistent infections in Ungulate, Felidae and primates. Primate lentiviruses are typified by the AIDS virus, HIV. Primate lentiviruses, closely related to HIV, termed SIV, have been identified in diverse wild monkey species in Africa. The recent lethal AIDS pandernics is in contrast with the non-pathogenic hostvirus relationship observed in SIV infected monkeys in the wild. However, SIV strains of increased virulence have been obtained through experimental cross-species transmissions. Dramatic changes of virulence for the same host species can be attributed to a limited number of genetic changes in the viral genome. Given the documented high variability of lentiviruses, the question arises of mechanisms explaining stability of the non virulent phenotype in the wild monkey populations. Indeed, multiple intra-species transmissions (i.e. from sooty mangabey to sooty mangabey) did not produce virulent variants, whereas one cross-species transmission (i.e. from mangabey to macaque) was sufficient to produce a variant which is pathogenic for both species. Counterselection of virulent variations is thus efficient in the adapted host species. The mechanism explaining such counterselection of virulence is not simply host death, which is not observed in largely infected wild monkey populations. Our observations support the existence of host-specific selection favoring new virulence phenotypes when an adapted virus is brought into a new host environment. Thus, in the case of SIV, virulence changes may be due to viral factors, modified through differential selection by host factors.
Epxression of acylated and fatty acid free influenza $\mathrm{HA}$ in insect cells - comparative receptor binding and fusion studies

H. Philipp *, M. Veit*, T. Korte**, A. Herrmann** and M.F.G. Schmidt Influenza HA mediates the adhesion and penetration of host cell membranes. Acylation of three conserved cysteine residues in the membrane spanning region of several HA subtypes has been shown. The biological significance of this hydrophobic modification remains to be elucidated. A possible role for the mem-brane fusing activity has been controversially discussed in the past. Removal of covalently bound farty acid from protein by incubation with hydroxylamine is a commonly applied method. As we shall show for a number of different envelope viruses with acylated glycoproteins, this treatment leads to quite different consequences in a functional sense depending on the vinus used. As an alternative approach to study the biological significance of palmitoylation we expressed wild-type influenza HA and an HAmutant lacking the fatty acid binding sites using the baculovirus system. Both wild type and $\mathrm{FA}^{-}$-mutant $\mathrm{HA}$, after expression in insect cells bind to red blood cells, induce hemolysis, and fuse efficiently with fluorescently labeled erythrocyte ghosts Hydroxylamine treatment of both recombinant wt- and mutant-HA leads to a loss of hemolysis and of fusion activity. At least in the case of FA-HA its inhibitory action must be related to some other effect than fatty acid cleavage. These results indicate that hydroxylamine treatment may not always be suitable for the generation of fatty acid free glycoproteins or virus particles for functional studies.

* Freie Universität Berlin, Inst.f.Immunol.u.Molekularbiol., Königin-Luise-Straße 49, D-14195 Berlin, Germany,

** Humboldt-Universität $\mathrm{zu}$ Berlin,Insti.f.Biophysik,Invalidenstraße 42, D-10117 Berlin, Germany.

\section{S04-32}

INFLUENZA A INFECTIONS AMONG TURKEYS CAUSED BY H1N1 SWINE INFLUENZA VIRUSES - NEW PATHWAYS OF INFECTION AND EEEECTS ON VIRAL EVOLUTION

S. Ludwig*, C. Scholtissek*, A. Haustein\# and E.F. Kaleta \# *Institut für Virologie, \#Institut für Geflügelkrankheiten, Justus-Liebig-Universität, D-35392 Giessen, Germany

In $1991 \mathrm{H} 1 \mathrm{~N} 1$ influenza A viruses were isolated from turkeys of three different farms located in the Netherlands and Northern Germany. Since one of the farms, near Bremen, is surrounded by agricultural land, were farmers distribute liquid manure from their pigs, swine viruses were incriminated as a possible source of the disease. This suggestion was proven by genetic analyses of a turkey virus and a corresponding swine influenza A virus isolated in the same region. Sequence comparison and construction of phylogenetic trees exibit a high homology between these two viruses. Additional analyses of other avian and swine isolates further provided us with information on the evolution of the North European H1NI swine viruses, establishing a new lineage, and their role as a pool for new pathogens. 


\section{Subcellular Signal Transduction}

S05-01

CHANGES IN MICROFILAMENT ORGANIZATION AFTER CALCIUMINDUCED GROWTH CONE COLLAPSE OF LEECH NEURONS M.D. Neely, M. Gesemann and J.G. Nicholls, Biocenter Univ. Basel, 4056 Basel, Switzerland

Depolarization of leech neurons leads to growth cone collapse and neurite Depolarization of leech neurons leads to growth cone collapse and neurite
retraction. This response to depolarization is infuenced by the substrate on retraction. This response to depolarization is influenced by the substrate on
which the cells are growing and is mediated by the influx of calcium (S. Grumbacher-Reinert and Nicholls, 1992, J. Exp. Biol. 167:1-14; Neely, 1993, J. Neurosci. 13:1292-1301). The morphological changes induced by depolarization of leech neurons growing on leech extracellular matrix extract is accompanied by a loss of microfilaments in the growth cones. Leech neurons growing on a different substrate, the plant lectin concanavalin A (ConA), did not retract their neurites or lose microfilaments, but continued to grow after depolarization. We attribute this to the reduced calcium influx during depolarization of neurons on ConA (Ross et al. 1988, PNAS 85:4075-4078). Increasing the intracellular calcium concentration by incubating neurons with the calcium-ionophore A23187, led to cessation of motility and disruption of microfilaments but not microtubules in growth cones on ConA. To investigate the mechanism by which calcium affects the organization of microfilaments we stained neurons with antibodies against gelsolin, a protein that severs microfilaments when it is activated with calcium. We have observed that these antibodies colocalize with microfilaments in leech growth cones. We are now analyzing the nature of this antigen that cross-reacts with the anti-gelsolin antibodies with the goal of establishing its potential role in the calcium-induced disruption of microfilaments in leech neuronal growth cones.

This work was supported by a grant from the Swiss Nationalfond No. 3127814.89 to J.G. Nicholls.

\section{S05-02}

\section{A NON-SPECIFIC CATIONIC CONDUCTANCE INDUCED BY ACTIVATION OF METABOTROPIC GLUTAMATE RECEPTORS IN CA3 PYRAMIDAL NEURONS}

Nathalie C. Guérineau, Beat H. Gähwiler and Urs Gerber

Brain Research Institute, August Forel-Strasse 1, CH-8029 Zürich

Activation of metabotropic glutamate receptors (mGluRs) was studied in voltage-clamped CA3 cells in rat hippocampal slice cultures using the whole-cell patch-clamp configuration. In saline containing $16 \mathrm{mM} \mathrm{K}^{+}$, 1S,3R-ACPD (50 $\mu \mathrm{M})$, a mGluR agonist induced an inward current associated with an increase in membrane conductance. The reversal potential, assessed with a voltage ramp protocol from -80 to $-60 \mathrm{mV}$ and calculated by linear regression, was $-15.7 \pm 18.7 \mathrm{mV}$, and was shifted to $-53.4 \pm 6.4 \mathrm{mV}$ when the concentration of external monovalent cations was halved. These results indicate that the conductance underlying this current is selective for cations (mainly $\mathrm{K}^{+}$and $\mathrm{Na}^{+}$). The steady-state $\mathrm{I} / \mathrm{V}$ curve for the $1 \mathrm{~S}, 3 \mathrm{R}$ ACPD-induced inward current showed a slight inward rectification. In most of the cells, this inward current was followed (or overlapped) by an outward current concomitant with a decrease in a $\mathrm{K}^{+}$conductance $\left(\mathrm{E}_{\mathrm{rev}}=-51.2 \pm 5.6\right.$ $\mathrm{mV}$ in $\left[\mathrm{K}^{+}\right]_{0}=16 \mathrm{mM}$ and shifted to $-74.0 \pm 2.8 \mathrm{mV}$ in $\left[\mathrm{K}^{+}\right]_{0}=8 \mathrm{mM}$ ). This $\mathrm{K}^{+}$current was voltage-independent in the membrane potential range of -120 to $-20 \mathrm{mV}$. Similar results were obtained with methacholine, a cholinergic muscarinic agonist. The non-specific cationic current, seen only in the presence of elevated extracellular $\mathrm{K}^{+}$concentration, could play an important role during intense neuronal activity.

\section{S05-03}

NEGATIVE CHARGE AT THE CASEIN KINASE II SITE FLANKING THE NUCLEAR TARGETING SIGNAL OF SV4O LARGE T-ANTIGEN ENHANCES ITS NUCLEAR TRANSEORT David A. Jans and Patricia Jans; Division for Biochem. \& Mol. Biol., John Curtin School of Medical Research, A.N.U., Canberra, Australia Nuclear import of SV40 large T-antigen fusion proteins is greatly enhanced by the casein kinase II (CKII) site (SSDDE111-115) flanking the nuclear targeting sequence (PKKKRKV132). We examined its role using site-directed mutagenesis of the site and analyzing the effects on nuclear import at the single cell level using quantitative fluorescent techniques. Inactivation of the site by deletion, substitution of ss111/112 by nonphosphorylatable amino acids, or mutation of the DDEI13/115 CKII recognition sequence markedly reduced nuclear import $<15 \%$ wild type - WT). Substitution of Asp for $\mathrm{S}^{112}$ (the serine predominantly phosphorylated by CKII) enhanced nuclear import to about $60 \%$ WT levels. we conclude that negative charge at the ckII site, normally provided by phosphorylation, is mechanistically important for nuclear transport enhancement. A direct role for phosphatases in the nuclear transport process can be discounted.
S05-04

REGULATION OF THE C-MYC PROTO-ONCOPROTEIN AND ITS PARTNERS

Bernhard Lüscher, Anna Bakardjiev, Kristine Bousset, Christa Cerni ${ }^{1}$ Marie Henriksson, Lars-Gunnar Larsson, and Juliane $M$. LüscherFirzlaff, Institut fŭr Molekularbiologie, MHH, D-30623 Hannover, and

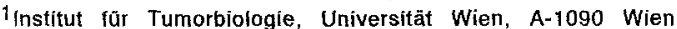

The c-Myc protein has been implicated in the regulation of cell growth and differentiation. C-Myc specifically interacts with Max which in turn forms heterodimers with Mad and Mxi1 and homodimers with itself. We present evidence that this network of proteins is regulated both at the level of relative protein concentration as well as by post-translationa mechanisms. In the hematopoietic cell lines U-937, ML-1 and HL-60 c-myc mRNA and protein are rapidly downregulated after induction of differentiation whereas max expression is not significantly altered. In contrast the expression of $\mathrm{mad}$ is induced with properties similar to immediate early genes. $m x i 1$ is induced to a lesser degree and with slower kinetics. In an effort to study the regulation of c-Myc we have mapped all the major in vivo phosphorylation sites in both c-Myc and Max. Two sites in the $\mathrm{N}$-terminal transactivation domain are important for the regulation of the transforming potential of c-Myc both in established cell lines as well as in primary rat embryo fibroblasts. However, no difference in the transactivating potential of corresponding mutants was observed. Phosphorylation of Max at sites close to the basic region increases both on- and off-rates of either Myc-Max hetero- as well as Max homodimers to DNA.

\section{S05-05}

GABA $_{3}$ AND ADENOSINE RECEPTORS MEDIATE ENHANCEMENT OF A SLOW $\mathrm{K}^{+}$CURRENT $\mathrm{BY}$ INHIBITING ADENYLYI, CYCLASE IN RAT CA3 CELLS Urs Gerber and Beat $\mathrm{H}$. Gähwiler Brain Research Institute, University of zürich, CH-8029 Zürich, Switzerland

$\mathrm{GABA}_{\mathrm{B}}$ and adenosine receptors act via pertussis toxinsensitive G-proteins of the $G_{i} / G_{o}$ class to mediate longgensitive Groteins of the $a_{i} / G_{o}$ class to mediate longlasting inhibition in the CNS. This class of G-protein is negatively coupled to the enzyme adenylyl cyclase. Our aim was to determine whether the inhibition of adenylyl cyclase mediated by these receptors has functional congequences for electrical signaling in hippocampal neurons. The calciumdependent $k^{+}$cuxrent, $I_{A H P}$ underlying adaptation of cell firing, was used as an electrophysiological bioassay for adenyly cyclase activity, as it is very gensitive to intracellular levels of cAMP. Accordingly, the $\beta$-adrenergic agonist isoproterenol $(5-500 \mathrm{nM})$, that activates $G_{\text {, }}$ inked receptors, reduced the amplitude of $I_{\text {ArP }}$ by $51.5 \pm 3.6 \%$ in volta pyramidal cells in TTX $(n=20)$. voltage-clamped CA3 pyramidal cells in $1 \mathrm{AX}(\mathrm{n}=20)$ Reduction of $I_{\text {AHP }}$ bY in the presence of baclofen $(10 \mu \mathrm{M})$, $2.9 \%$, ( $p<0.001$ ) in the presence of baclofen
acting at $\mathrm{GABA}_{\mathrm{B}}$ receptors, or adenosine $(50 \mu \mathrm{M})$.

acting at $\mathrm{GABA}_{\mathrm{B}}$ receptors, or adenosine $(50 \mu \mathrm{M})$. Thus, the inhibition of adenylyl cyclase activity due to responses to other neurotransmitters by an interaction ccurring at the level of intracellular signal trangduction. Furthermore, stimulation of these receptors will tend to enhance $I_{\text {AHP }}$, suppressing repetitive cell firing. This represents a further mechanism which will result in prolonged inhibition of hippocampal neurons.

\section{S05-06}

\section{NITRIC OXIDE MODULATES ENDOGENOUS DOPAMINE} RELEASE IN BOVINE RETINA

Olivier Bugnon ${ }^{1}$, Nicolas C. Schaad ${ }^{2}$, Michel Schorderet ${ }^{1}$ Department of Pharmacology, ${ }^{1}$ University Medical Center, 1211 Geneva, 2 Division of Clinical Psychopharmacology, Institutions Universitaires de Psychiatrie de Genève, 1225 Chêne-Bourg, Switzerland.

A study of the possible modulation by nitric oxide (NO) of endogenous dopamine (DA) release was performed in bovine retina in vitro. Isolated half retinas were incubated in KRG at $37^{\circ} \mathrm{C}$ for 3 successive $30-\mathrm{min}$ incubations, and DA was measured in the incubation medium by HPLC with $\mathrm{EC}$ detection. When retinas were incubated in the presence of the NO- generating compound hydroxylamine $(300 \mu \mathrm{M})$, a decrease in either basal or $\mathrm{K}^{+}-(50 \mathrm{mM})$ stimulated DA release was observed during the $2^{\text {nd }} 30$-min period. Tested under similar conditions, dibutyryl cGMP (1 $\mathrm{mM}$ ) was ineffective. The NO synthase inhibitor $L-\mathrm{N} G_{\text {-nitro-arginine }}$ methyl ester ( $L$-NAME, $100 \mu \mathrm{M}$ ) increased basal DA release during the $1^{\text {st }}$ and $2^{\text {nd }}$ incubation, whereas it did not affects the $\mathrm{K}+$-stimulated DA release. In addition, its effect were potentiated in calcium-free medium. Finally, we have also shown in cell-free experiments that retinal NO synthase activity was totally calcium-dependent and blocked by $L$ NAME. Taken together, these results suggest that endogenous NO regulates DA release $v i a$ a cGMP independent mechanism 
S05-07

CAPACITATIVE CALCIUM INFLUX AND ACTIVATION OF STEROIDOGENESIS BY ANGIOTENSIN II IN ADRENAL GLOMERULOSA CELLS

M.F. Rossier, M.M. Bumay, C.P. Python, M.B. Vallotton, and A.M. Capponi Division of Endocrinology, University Hospital, CH-1211 Geneva 14 While the stimulation of aldosterone biosynthesis by angiotensin II (AngII) in bovine glomerulosa cells clearly depends upon a sustained influx of $\mathrm{Ca}^{2+}$, the pathway for $\mathrm{Ca}^{2+}$ entry is not yet accurately defined. At least two mechanisms seem to be involved: 1) the opening of voltage-operated $\mathrm{Ca}^{2+}$ channels, and 2) the stimulation of a "capacitative" $\mathrm{Ca}^{2+}$ entry regulated by intracellular $\mathrm{Ca}^{2+}$ stores; however the relative contribution of these pathways to the stimulation of steroidogenesis needs to be determined. Two pharmacological tools were used: nicardipine, a blocker of $\mathrm{T}$ - and L-type $\mathrm{Ca}^{2+}$ channels, and thapsigargin, which releases $\mathrm{Ca}^{2+}$ and therefore induces a capacitative $\mathrm{Ca}^{2+}$ influx. Nicardipine (1 $\mu \mathrm{M})$ completely blocked the cytosolic $\mathrm{Ca}^{2+}$ response to $\mathrm{K}^{+}$, which exclusively activates voltage-operated $\mathrm{Ca}^{2+}$ channels, but only partially $(22 \%)$ the response to AngII. In the presence of nicardipine, AngII was unable to stimulate a $\mathrm{Ca}^{2+}$ influx after depletion of $\mathrm{Ca}^{2+}$ stores with thapsigargin, demonstrating that AngII principally stimulates a capacitative $\mathrm{Ca}^{2+}$ entry pathway. In addition, nicardipine completely blocked the steroidogenic response to $\mathrm{K}^{+}$, but only $30 \%$ of the response to Angll. Thapsigargin-induced aldosterone formation, as the response to AngII, was markedly potentiated by low concentrations of $\mathrm{K}^{+}$. In conclusion, this study shows that in bovine glomerulosa cells, AngIl activates a sustained $\mathrm{Ca}^{2+}$ influx, principally through a capacitative pathway, leading to aldosterone formation. This finding could partially explain the poor efficiency of dihydropyridine $\mathrm{Ca}^{2+}$ antagonists for preventing aldosterone secretion.

\section{S05-08}

Heat-shock induced activation of MAP-kinases in HeLa cells

Harald Heider and John M. Lucocq*. Institute of Anatomy, University of Berne, CH 3012 Beme *Department of Anatomy and Physiology, University of Dundee, UK Dundee DD1 4HN.

Subjection of Hela cells to $45^{\circ} \mathrm{C}$ for 30 minutes leads to a complex activation pattern of MAP-kinases as revealed with renaturation gels. Exponentially growing HeLa cells show activation of a myelin basic protein (MBP) phosphorylating kinase migrating with an apparent molecular mass of around $60 \mathrm{kDa}$ upon heat shock induction. In contrast, in cells arrested in Go by serum starvation for 24h, essentially three different proteins phosphorylating MBP are activated subsequent to heat shock treatment. These kinases migrate with apparent molecular masses of around $32 \mathrm{kDa}, 44 \mathrm{kDa}$ and $55 \mathrm{kDa}$. Westem blot analysis with antibodies recognizing the human homologues of erk1 and erk2 revealed that considerable pools of erk1 and erk2 show retarded electrophoretic migration behaviour indicating that they are phosphorylated and probably activated in heat subjected and growth-arrested HeLa cells.

Down-regulation of protein kinase $C(\mathrm{PKC})$ by a prolonged treatment with 12 O-tetradecanoyl-phorbol-13-acetate (TPA) did neither change the major activation pattern observed upon separation of cell lysates on renaturation gels nor that one observed on westem blots decorated with anti-erk1 or anti-erk2 antibodies. These results suggest that heat shock induced activation of at least erk1, erk2 and the 55kDa MBP-kinase is not mediated via activation of PKC This work was supported by Swiss NSF grant no. 31-27636.89.

\section{S05-09} Mechanisms of glucocorticoid receptor desactivation by
homopolymeric alanines

Martin Hug, Manuela Höfferer and Sandro Rusconi, Institut für Molekularbiologie II der Universität UZ Zürich, Winterthurerstrasse 190, 8057 Zürich, Switzerland

The rat glucocorticoid receptor (GR) cDNA contains in its 5'-portion a (CAG-repeat that encodes a string of 22 Gln residues interrupted by $1 \mathrm{Arg}$. A mutant with the frame-shifted repeat which codes now for poly-Ala residues (called GR[Ala]) is completely trans-inactive, is more cytoplasmically located in absence of ligand and exhibits dominant-negative properties in presence of ligand (Lanz et al., Steroids, in press). We generated GR recombinants with increasing numbers of CAG-Triplets in three possible reading frames (oligo-Gln, -Ser or -Ala). The GR-oligo[Ala] $\leq 23$ mutants showed an activity comparable to wild type GR, whereas the GRoligo[Ala] $\geq 25$ were inactive. The severity of the effect of oligo Ala strings seems to be dependent on the position of the repeat along the primary sequence (e.g. progressive decrease of the effects by moving toward more carboxy-position). We have also examined the effects of the oligo[Ala]21, $23,25,27,31$ on GAL 4 chimeric factors and found that the inhibition by Ala repeats is probably factor-specific. Our results made us speculate that possible differences in post-translational modifications may be at the root of the negative effects by the Ala repeats. We believe that these effects are due to transient association of the nascent GR[Ala] mutant proteins with intracellular membranes. These hypotheses are currently tested.
S05-10

Effects of dominant negative glucocorticoid receptor mutants in cell cultures and transgenic animals

Stefano Brenz Verca, Stefan Schneider, Sandro Rusconi, Institut für Molekularbiologie II der Universität Zürich, Winterthurerstr. 190, CH8057 Zürich, Switzerland

The glucocorticoid receptor (GR) is involved in a large number of developmental and biochemical processes. Recently, we obtained a trans-dominant negative GR mutant (TDN) - called GR[Ala] - by a frame shift of the CAG-repeat of the rat GR (see poster by Hug et al.). The GR[AIa]-mutant shows normal ligand-binding and DNA-binding but cannot stimulate transcription (Lanz et al., Steroids; in press). Modulatable versions of the TDN-mutant have also been created by substituting the HBD of the GRmutant with the HBD of sex-hormone receptors. These constructs have only been tested so far in transient cotransfection assays. Therefore, we are currently trying to express them permanently in cell lines (rat hepatoma cell line FTO-2B) in order to verify whether they can affect the transcription level of a known endogenous GR-dependent target-gene, like the tyrosine aminotransferase (TAT) gene or a permanently transformed MTV-lacZ reporter. So far no rapid test-system has been described, that allows the quantitative analysis of such effects in transient expression assays. We are about to set up such a rapid and generally applicable transient test-system. In the future we plan to express dominant negative GR mutants in a tissuespecific manner in transgenic mice. It will be particularly interesting to investigate their effects on the immune system or on liver functions.

\section{S05-11}

\section{MODEL GUIDEPOSTS DIRECT GROWTH CONE PATHFINDING BY ACTIVATING SECOND MESSENGER}

T.B. Kuhn, M.F. Schmidt and S.B. Kater, Dept. of Anatomy and Neurobiology, Colorado State University, Fort Collins, CO 80523 , USA

These studies investigated growth cone responses to brief local encounters with surface molecules implicated in neuronal pathfinding using chick DRG neurons. We tested two interrclated hypotheses: 1) Discrete points of information (guideposts) override sustained surfacc information and dominate growth cone behavior and 2) guidance molecules provide such information by activating second messengers instead of simply increase adhesivity. The potential guidance molecule laminin was covalently coupled to polystyrene beads in order to mimic guidepost cells. Encourters with laminin model guideposts significantly altercd the behavior and morphology of growth cones advancing on fibronectin or polylysine. Growth cones steered towards theses beads in a saltatory motion once a long lasting contact with a single filopodium had been established. Subsequent to a pause at the bead, growth cones advanced with a significantly increased growth rate for the next $11 \mathrm{~min}$. The average increase in rate stimulated by bead contact was 5 times the basal rate on polylysine and 2.5 times that on fibronectin. Positioning of multiple model guideposts along a path and within filopodial reach maintained this bead stimulated rate of outgrowth and directed growth cone advance. The laminin induced pattern of events was totally blocked in the presence of either $\mathrm{H} 7$ or sphingosine, two PKC inhibitors, or Neomycin, a PLC inhibitor. Neither of these inhibitors affected neurite outgrowth on the substrata alone. Our results demonstrate the necessity of filopodial contacts, the importance of spatially restricted stimuli and the activation of transducing pathways as key mechanisms in neuronal pathfinding.

\section{S05-12}

The Activation of Steroidogenesis in Calcium-Clamped Bovine Glomerulosa Cells and its Modulation by Angiotensin II and CAMP.

Python CP, Laban OP, Rossier MF, Vallotton MB and Capponi AM

Division of Endocrinology, University Hospital, Geneva, Switzerland. The $\mathrm{Ca}^{2+}$-messenger system plays a crucial role in the regulation of steroid secretion in adrenal zona glomerulosa cells, as it is known to mediate the action of angiotensin II and potassium ion. In the present study, we used intact isolated glomerulosa cells in which the cytosolic free $\mathrm{Ca}^{2+}$ concentration $\left(\left[\mathrm{Ca}^{2+}\right]_{c}\right)$ was clamped at various levels with the $\mathrm{Ca}^{2+}$-ionophore, ionomycin, in order to locate the sites of action of $\mathrm{Ca}^{2+}$, to determine their sensitivity towards $\mathrm{Ca}^{2+}$ and the modulation by other physiological factors. By measuring simultaneously steroid synthesis and $\left[\mathrm{Ca}^{2+}\right]_{\mathrm{C}}$, we show that $\mathrm{Ca}^{2+}$-clamping (50-860 $\mathrm{nM})$ induced a concentration-dependent increase in the production of

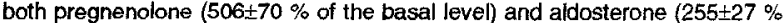
of the basal level, $\mathrm{EG}_{50}=273 \mathrm{nM}$ ). By contrast, $\mathrm{Ca}^{2+}$ did not influence the conversion of 11-deoxycorticosterone into aldosterone at concentrations up to $600 \mathrm{nM}$, although at higher concentrations we observed a modest but significant inhibition. In addition, $\mathrm{Ca}^{2+}$-clamping did not affect the formation of pregnenolone from a freely diffusible analogue of cholesterol, 25. hydroxycholesterol, indicating that $\mathrm{Ca}^{2+}$ acts at a step upstream of cholesterol side-chain cleavage. Both angiotensin 11 and a membrane-permeant surrogate of CAMP, dibutyryl cyclic AMP, potentiated the steroidogenic response to increases in $\left[\mathrm{Ca}^{2+}\right]_{c}$. In summary, these studies reveal an exquisite sensitivity of the glomerulosa cell steroidogenic pathway toward very small physiological changes in $\left[\mathrm{Ca}^{2+}\right] \mathrm{c}$. 
S05-13

THE TRANSCRIPTION FACTOR MTF-1 IS ESSENTIAL FOR BASAI AND HEAVY METAL-INDUCED METALI OTHIONEIN-I GENE EXPRESSION

Freddy Radtke, Rainer Heuchel, Oleg Georgiev, Gerlinde Stark\#, Michel Aguett \& Walter Schaffner

Institut für Molekularbiologie II, Universität Zürich, 8057 Zürich

\# Institut für Molekularbiologie I, Universität Zürich, 8093 Zürich

We have previously described and cloned a factor (MTF-1) that binds specifically to metal-responsive DNA sequence elements in the enhancer/promoter region of metallothionein genes. MTF-1 is a protein of $72.5 \mathrm{kDa}$ that contains six zinc fingers and multiple domains for transcriptional activation. Here we report the disnuption of both alleles of the MTF-1 gene in mouse embryonic stem cells by homologous recombination. The resulting null mutant cell line fails to produce detectable amounts of MTF-1. Moreover, due to the loss of MTF-1 the endogenous metallothionein-I gene is silent, showing that MTF-1 is required for both basal and metal-induced transcription. Accordingly, reporter genes containing either synthetic or natural metal-responsive promoters were not transcribed after transfection into the null mutant cells. Cotransfection of the MTF-1 expression vector rescued both basal and heavy metal-induced transcription. These results demonstrate that MTF-1 is essential for normal metallothionein gene regulation.

\section{S05-14}

INDUCTION OF FAST NEURITE RETRACTION BY THE PHOSPHATASE INHIBITOR CALYCULIN-A ON NGF-DIFFERENTIATED RAT PHEOCHROMOCYTOMA (PC12) CELLS

B. Reber, A. Bouron \& H. Reuter; Department of Pharmacology, University of Bern, CH-3010 Bem

Rat pheochromocytoma cells (PC12) were differentiated with NGF for 3-4 days, a time sufficient for the formation of growth cones and elongation of neurites. Addition of the phosphatase inhibitor calyculin A (CL-A) $(0.2-50.0 \mathrm{nM})$ led to a concentrationdependent collapse of growth cones and retraction of the neurites within 15 minutes. After the retraction, the cell bodies showed the appearance of a grape-like domain opposite to the cell nucleus. They recovered to normal shape within about $30-60$ minutes if the inhibitor was washed out. The neurite retraction was further analysed using both low-light level fluorescence video-microscopy and fura-2 single cell microfluorimetry. The onset of retraction started in the central part of the growth cone prior to a complete collapse of filopodia. The basal intracellular free calcium concentration $\left(\left[\mathrm{Ca}^{2+}\right]_{1}\right)$ remained constant during neurite retraction. As a measure for the viability of CL-A-treated cells, agonist-induced responses of $\left[\mathrm{Ca}^{2+}\right]_{i}$ were analysed. $\mathrm{Ca}^{2}+$ entry across voltage-activated $\mathrm{Ca}^{2}+$ channels was inhibited by $50 \%$ after 30 minute treatment with $\mathrm{CL}-\mathrm{A}$, while the $\mathrm{ATP}$-induced $\mathrm{Ca}^{2+}$ entry via $\mathrm{Ca}^{2+}$-permeable cation channels was not significantly reduced. However, the onset of the ATP-induced rise in $\left[\mathrm{Ca}^{2+}\right]_{i}$ started at the grape-like domain. The speed of the $\mathrm{Ca}^{2}+$ wave across the cell body was slower than in control cells. The addition of $0.5 \mu \mathrm{M}$ okadaic acid, a different type of phosphatase inhibitor, caused no cell shape change or neurite retraction within 24 hours. Taken the specificity of the two phosphatase inhibitors into account. the observed effects seem to be induced by inhibition of a PPl-class phosphatase.

\section{S05-15}

A NEN S10O PROTEIN TRAALLLOCATED TO THE MEMERANE OF HUMAN ACITVATED NEUITOEHILS SIMILARLY TO MRP-B AND MRP-14.

Guignard F., Vaglio M., Mauël J.* and Markert M., Iaboratory of Clinical Gemistry, oHV and Biochemistry Institute*, aH1011 Iausanne.

A new calcium-binding protein of $6.5 \mathrm{kDa}$ (p6) belonging to the S100 family was identified in human neutrophils by Nteminal amino acid sequencing. Using two dimentional electrophoresis, $p 6$ was shown to exist as two isoforms of $p I$ 6.2 and 6.3. Under calcium-dependent activation, the two isoforms of $p 6$ appeared to translcate to the membrane, but were evidenced only in the cytosol with calcium-independent stimulation in parallel to the other S100 proteins, MRP-14 and MRP-3. The po protein did not appear phosphorylated and no calcium-binding was evidenced by calcium overlay. In conclusion, a new $\$ 100$ protein was identified and was found to translocate to the same extent as MRP-14 and MRP-8 suggesting an identical regulation.
S05-16

HYPOXIA AND COBALT STIMULATE LACTATE DEFYDROGENASE ACTIVITY IN VASCULAR SMOOTH MUSCLE CELLS

Marti H., Jung H., *Pfeilschifter J., Bauer C., Physiologisches Institut, Universität zürich und *Biozentrum, Abt. Pharmakologie, Basel

oxygen plays a dominant role in the metabolism and viability of cells. Hypoxia induces the transcription of a number of genes, such as the glycolytic enzymes. We have investigated signalling events that may lead to an enhanced activity of $\mathrm{LDH}$ in cultured vascular smooth muscle cells (VSMC) of rat aorta grown under hypoxic conditions. LDH was chosen because this enzyme exhibits the largest increase in activity among all glycolytic enzymes when cells become hypoxic. Upon incubation of VSMC for $24 \mathrm{~h}$ at $1 \%$ oxygen, a 2-fold increase in LDH activity was observed, compared to cells kept at $20 \% \mathrm{O}_{2}$. Hypoxic incubation led in parallel to a 2.5-fold increase in intracellular cAMP levels. A dose dependent increase in LDH activity was also observed in cells incubated at $20 \% \mathrm{O}_{2}$ in the presence of cobalt chloride, indicating that a metal binding protein might be involved in this signalling cascade.

S05-17

Myosin in nematocytes of Hydra

Michel Nakano \& Robert P. Stidwill

Dept. Zoology, University of Zürich, Switzerland

The functions of nonmuscle myosins during cell locomotion are not clear. Several models have been presented reaching from very active participation of the molecules in different parts of cells to only minor or no roles at all. There is increasing evidence that the functions of myosin II (capable of forming bipolar filaments) are quite distinct from the ones of myosin I and generally that the different members of the growing family of myosin-like proteins may not all have identical functions.

We have attempted to demonstrate the occurrence and determine pattern of myosins in tissue of Hydra and especially in migrating nematocytes mainly for two reasons. First, nematocytes are fairly rapidly moving cells which can be studied in vitro and, second, the question is of interest from a evolutionary point of view.

We have utilized several antibodies against vertebrate and invertebrate myosins for immunofluorescence (confocal laser scanning microscopy) and Western blotting studies. In addition to these results findings on the screening of a 2 ZAP c-DNA library are presented.

\section{S05-18}

NUCLEOLAR LOCALIZATION OF THE JAK1 PROTEIN TYROSINE RINASE A. Ziemiecki, G. Zürcher, A-C. Andres, A. Harpur ${ }^{\circ}$ and A.
Wilks

Abteilung für klinisch-experimentelle Forschung, Universität Bern, Tiefenaustrasse 120, 3004 Bern. oudwig Institute for Cancer Research, Melbourne, Australia

The JAKl protein tyrosine kinase is a nember of a family The JAK 1 protein tyrosine kinase is a member of a family kinase catalytic domains: a bona fide tyrosine kinase domain and an amino terminaliy positioned kinase-related domain. Recently it has been shown that JAK1 and the other members of this family (JAK2, TYK2) are intimately inyolved in cytokine and growth factor signalling. The presence of two putative nuclear localizing sequences in the amino terminus of the protein prompted us to examine the subcellular localization of this protein. Immunohistochemical and biochemical analysis revealed mat a that a part of JAK1 was localized to the nucleolus. Metabolic labelling showed that (a) the JAK1 protein partitioned rapidiy into the nucleus and (b) the nuclear JAK1 became smaller with time. The size difference was not due to either co-transiational glycosylation or phosphorylation. These results suggest that part of the JAK1 protein is rapidly translocated to the nucleoli where it becomes processed. 
S05-19 FAMILY OF PROTEIN TYROSINE KINASES

M. Flück, G. Zürcher, A-C. Andres and A. Ziemiecki Universität Bern, Tiefenaustrasse 120, 3004 Bern the tyrosine kinase domain, we have isolated a cDNA clone related to the $s y k / z a p 70$ family of intracellular PTKs. Northern blot analysis of RNA isolated from mouse organs revealed that this clone is expressed predominantly in spleen, mammary gland and thymus as two transcripts, a major species of approximately $5 \mathrm{~kb}$ and minor species of approximately $4 \mathrm{~kb}$. Nucleotide sequence gene. The syk gene is expressed as a major $3.0 \mathrm{~kb}$ transcript and has been reported to be lymphoid specific Due to the discrepancy of transcript size and and size and number, we suggest that our CDNA clone represents a novel member of this family of PTKS. We are currently isolating a full length sequence from a mouse spleen celI(s) in the mammary gland are responsible for the cell(s) in the mammar.
observed expression.
CHARACTERIZATION OF A NOVEL MEMBER OF THE SYK/ZAP70 Abteilung für klinisch-experimentelie Forschung,

Universität Bern, Tiefenaustrasse 120, 3004 Bern
Using a PCR cloning strategy and degenerate using a PCR cloning strategy and degenerate analysis revealed a $84 \%$ homology to the porcine $5 y k$

S05-22

DIFFERENTIATION AFFECTS G-PROTEIN PATTERNS AND G-PROTEIN-MEDIATED SIGNALING IN ERYTHROLEUKEMIA CELLS

F. Kesselring, K. Spicher* and H. Porzig, Departments of Pharmacology at the University of $\mathrm{CH}-3010$ Bern and at the *Freie Universität D-14195 Berlin

Expression and function of G-protein isoforms were studied in differentiating (6C8) and nondifferentiating (G3) subclones of RED-1 Rauscher murine erythroleukemia cells. Erythroid differentiation induced by the combined action of erythropoietin (Epo) and DMSO was associated with a selective loss $(>70 \%)$ of immunoreactive Goi3. Simultaneously, a truncated form of Goi2 accumulated in the cytosol, while the membrane concentrations of $\mathrm{G} \alpha \mathrm{i} 2$, Gos and $\mathrm{Goq} / 11$ remained unchanged.

Nondifferentiating G3 cells contained significantly higher levels of most $\mathrm{G} \alpha$ subunit isoforms that remained unchanged upon Epo/DMSO treatment. Changes in adenylyl cyclase activity and in intracellular $\mathrm{Ca}$ ion levels $([\mathrm{Ca}] \mathrm{i})$ resulting from activation of $\mathrm{G}$-protein-coupled receptors were monitored for differentiation-dependent effects on transmembrane signaling. ADP-mediated changes of [Ca]i in native and differentiating $6 \mathrm{C} 8$ cells were consistent with $\mathrm{P}_{2} \mathrm{~T}$ receptor coupling to $\mathrm{G} \alpha \mathrm{i} 3$.

Thrombin receptors seemed to couple preferentially to Gai in $\mathrm{G} 3$ cells and to $\mathrm{G} \alpha \mathrm{q}$ in $6 \mathrm{C} 8$ cells. Our results document substantial alterations in G-protein- mediated signaling during erythroid differentiation.

S05-23

INVOLVEMENT OF EPH-REIATED RECEPTOR PROTEIN TYROSINE D. Albrecht, H. Reid, G. Zürcher, A. Ziemiecki and A-C.

Andres Abteilung Fur klinisch-experimentelle Forsch To investigate the involvement of protein tyrosine To investigate the involvement of protein tyrosine kinases (PTKS) in the growth control of the mammary gland, we have used PCR based cloning to identify PTKs expressed during normal mammary gland development. This approach led to the isolation of three members of the eph-related family of receptor PTKs: $m y k-1$, a novel member expressed predominantly in lung, heart and mammary gland; $m$-eck, the murine homologue of the human eck gene and mek5, the murine homologue of the chicken cek5. All three PTKs were expressed in a distinct and narrow window of mammary gland development: puberty and estrus cycle. No expression was found either in late pregnancy or in the end differentiated state of pregnancy or in the end differentiated state of undifferentiated and invasive tumors of transgenic mice undifferentiated and invasive tumors of transgenic mice expressing the H-ras oncogene. In contrast, no elevated expression of all three genes was detected in the well
differentiated, non-invasive mammary tumors characteristic of $C$-myC expressing transgenic mice. These results suggest that members of this family of PTKs are involved in the control of proliferation of the mammary gland but are incompatible with its differentiation.

S05-21

$\begin{array}{lllll}\text { CELL-TYPE SPECIFIC ACTIVITY OF THE } & \text { RAT } \\ \text { ONCO- MODULIN LTR PROMOTER AS REVEALED BY }\end{array}$ ONCO- MODULIN LTR PRO
IN VIVO FOOTPRINTING

Jürg $M$. Rentsch, and Martin $W$. Berchtold. Institute of Veterinary Biochemistry, University of Zurich, 8057 Zurich, Switzerland.

By germline insertion, a long terminal repeat (LTR) of an intracisternal A-particle (IAP) endogenous retrovirus has overtaken the transcriptional control of the rat oncomodulin (OM) gene, which codes for a high affinity $\mathrm{Ca}^{2}{ }^{+}$ binding protein with modulatory capacity. Transient transfections with this LTR in combination with a reporter gene (hGH) showed no cell type specificity. In vivo sequencing showed a good correlation between $O M$ gene expression and hypomethylation. By DNase I hypersensitivity mapping and by in vivo DMS-footprinting we identified several cis elements in the OM LTR promoter which were only protected in the expression positive cell line. Analysis of protein, DNA interactions revealed the presence of a major, ubiquitously expressed LTR binding protein of $\mathrm{Mr} 100 \mathrm{kD}$ with a recognition sequence found to be protected in vive in a cell type specific Eashion.
CHARACTERIZATION OF THE LYMPHOCYTE SPECIFIC TRANSCRIPTION FACTOR NP-TCII.

Rusterholz, C., Espel, E., Reichenbach, P., Bucher, P. and Nabholz, M.

ISREC, 1066 Epalinges.

NP-TCII is a lymphocyte specific transcription factor (Lattion A.-I. et al., Mol. Cell. Biol., $1992,12: 5217-5227)$. Although it binds to the $x B$ site of the $5 V 40$ enhancer, it is unrelated to Rel-proteins. NP-TCII can be renatured after SDS-PAGE. It consists of a single type of polypetide chain with a heterogeneous apparent $\mathrm{MW}$ of $100-150 \mathrm{kDa}$. Limited trypsin digestion gives rise to two DNA-binding fragments of 40 and $44 \mathrm{kDa}$. Both have the same specificity as native NP-TCIT. With a novel algorithm for the analysis of bandshift data we have derived the consensus binding site AAGANTTTCC. Using partiaily purified protein we have screened 5 . flanking regions of various genes with $2-D$ bandshift assays and have confirmed the predicted consensus sequence.

S05-24

MOLECULAR CLONING OF A NOVEL SERINE/THREONINE KINASE WITH HOMOLOGY TO CELL CYCLE PROTEINS Millward, T. , Cron, P. , and Hemmings, B. A. Friedrich Miescher-Institut, P.O. Box 2543, CH4056 Base]

We have cloned a novel human protein kinase (termed oun kinase) with homology to certain yeast cell cycle proteins. The predicted amino acid sequence shows a $54 \mathrm{kD}$ serine/threonine kinase with an unusual catalytic domain structure, in which subdomains VII and VIII are separated by 30 residues. The only other known kinases with a similar primary structure are the 5 . cerevisiae proteins DBF2, DBF20 and CDC7. A consensus bipartite nuclear localization signal is present in the $\mathrm{NH}_{2}$-terminal domain of pun kinase. The Drosophila homologue is a protein of $53 \mathrm{kD}$, and is encoded by a gene located at $3 \mathrm{~L}$ $76 \mathrm{C}$. Current efforts are directed towards analysis of mutant phenotypes mapping to the same region. Additionally, peptide-specific antibodies have been prepared and are being used to characterize the protein in mammalian cells. 
S05-25

EXTRACELLULAR ATP AND UTP STIMULATE MITOGEN-ACTIVATEDPROTEIN (MAP) KINASE IN MESANGIAL CELLS

Andrea Huwiler and Josef Pfeilschiffer, Dept. Pharmacology, Biocenter, University of Basel, $\mathrm{CH}-\mathbf{4 0 5 6}$ Basel

Extracellular ATP and UTP activate a nucleotide receptor that mediates phosphoinositide and phosphatidylcholine hydrolysis by phospholipases $C$ and D, respectively. Here we report that ATP and UTP potently stimulate mesangial cell proliferation. Both nucleotides stimulate phosphorylation and activation of MAP kinase and the up-stream MAP kinase kinase. Activation of MAP kinase by both nucleotides is dose-dependently attenuated by the $P_{2}$ receptor antagonist suramin. Stimulation of protein kinase C (PKC) by phorbol ester increased MAP kinase phosphorylation and activation, and downregulation of PKC partially inhibited ATP- and UTP-induced MAP-kinase activation. Inhibitors of PKC which display a selectivity for the $\mathrm{Ca}^{2+}$-dependent isoenzymes $(\alpha, \beta, \gamma)$, as compared to the $\mathrm{Ca}^{2+}$-independent isoenzymes $(\delta$, $\varepsilon, \zeta, \eta)$ such as staurosporine and the specific PKC inhibitor CGP 41251 did not inhibit mucleotide-stimulated MAP kinase phosphorylation, thus implicating the involvement of a $\mathrm{Ca}^{2+}$-independent $\mathrm{PKC}$ isoform. In conclusion these data suggest, that ATP and UTP trigger the activation of the MAP kinase signalling cascade in mesangial cells and this may be responsible for the potent mitogenic acitivity of both nucleotides.

S05-26

\section{SUBCELLULAR ION-GRADIENTS IN CA SIGNALING}

P. Lipp \& E. Niggli, Deparment of Physiology, University of Bern

Recent experimental evidence has indicated an important role for submicroscopic concentration gradients during Ca-signaling in various cell types. In heart muscle cells influx of $\mathrm{Ca}$ via L-type $\mathrm{Ca}$ channels triggers $\mathrm{Ca}$ release from the sarcoplasmic reticulum (SR) and links electrical excitation to mechanical contraction (ec-coupling). We used a ratiometric confocal method (Fluo-3 and Fura-Red) to follow the intracellular $\mathrm{Ca}$-concentration while Na- and Ca-currents $\left(\mathrm{I}_{\mathrm{Na}}, \mathrm{I}_{\mathrm{Ca}}\right)$ were elicited in the whole cell mode of the patch-clamp technique. Both, $\mathrm{I}_{\mathrm{Ca}}$ and $\mathrm{I}_{\mathrm{Na}}$ were able to trigger $\mathrm{Ca}$ release Li-substitution experiments and the existence of a residual $\mathrm{I}_{\mathrm{Na}}$-induced $\mathrm{Ca}$ transient in the absence of SR function suggested that these $\mathrm{Ca}$ signals were mediated by the $\mathrm{Na}-\mathrm{Ca}$ exchange running in the $\mathrm{Ca}$ influx mode. Control experiments showed that $\mathrm{I}_{\mathrm{Na}}$-induced $\mathrm{Ca}$ transients did not result from spurious activation L-type $\mathrm{Ca}$ channels. The significant increase of the Na concentration required to activate the $\mathrm{Na}-\mathrm{Ca}$ exchange can only occur provided cytosolic diffusion of $\mathrm{Na}$ is restricted in the vicinity of the exchangers. This limited diffusion results in significant Na-gradients in the subsarcolemmal space. In conclusion, $\mathrm{I}_{\mathrm{Na}}$-induced $\mathrm{Ca}$ influx may represent a safety factor for ec-coupling while $\mathrm{I}_{\mathrm{Ca}}$ ensures sustained Ca-release and is the source for refilling the SR with $\mathrm{Ca}$.

Supported by SNF. from the $\mathrm{SR}$. Kinetic differences between $\mathrm{I}_{\mathrm{Na}}$ - and $\mathrm{I}_{\mathrm{C}}$-induced Ca-transients,

\section{S05-28}

ROLE OF PHOSPHATIDYLINOSITOL 3-KINASE (PI3-K) IN GLUCOCORTICOID-INDUCED INSULIN RESISTANCE Assimacopoulos-Jeannet F., Guillaume-Gentil C., Le Marchand-Brustel Y., Jeanrenaud B. Laboratoires de Recherches Métaboliques, Geneva, Switzerland.

Glucocorticoids induce hepatic + muscle insulin resistance, by an unkown mechanism. Several steps in the insulin signalling pathway have been recently described. A possible interference of glucocorticoids with insulin signalling was measured in muscles and liver. The data show that although glucocorticoids inhibit PI13-K activity in some muscles, this inhibition cannot explain glucocorticoidinduced insulin resistance and that effects further down in the signalling pathway have to be investigated. Such a defect could play a role in hepatic resistance.

\section{S05-29}

HACAT CELLS IN CULTURE ARE UNABLE TO EXPRESS LORICAIN, A LATE DIFFERENTIATION MARKER

M. Huber, F. Amiguet-Barras, D. Breitkreutz+, C. Backendorf \&, D. Hoh 1, Dermatology, CHUV, 1011

Lausanne, +German Cancer Research Center, 6900

Heicielberg; §Department of Biochemistry, University of Leiden, Leiden.

The HaCaT cell line is an immortal, nontumorigenic human keratinocyte line. Transplants of HaCaT celis onto nude mice reform an orderly structured and differentiated epidermal tissue. To study the epidermal differentiation of this ine we used the following 5 genes: involucrin, sprr $1-3$ and loricrin. These genes are sequentially expressed during keratinocyte differentiation, and their proteins are important precursors for the cornified cell envelope. HaCaT cells were cultured under different conditions known to promote keratinocyte differentiation. Expression of the genes was detected by Northern blot analysis. In HaCaT cells no loricrin expression was detectable while the other genes were all expressed. Surprisingly, RNA from a transplant of activated c-Ha-ras transfected HaCaT cells showed good expression of all 5 genes. At the molecular level, the HacaT cell line might therefore not be a good in-vitro model for late stages of epidermal differentiation.

\section{S05-27}

\section{SPATIO-TEMPORAL FEATURES OF CALCIUM SIGNALING IN CARDIAC MUSCLE CELLS}

E. Niggli \& P. Lipp, Department of Physiology, University of Bern

The regenerative $\mathrm{Ca}$-induced $\mathrm{Ca}$ release (CICR) mechanism is an important amplifier in cardiac signal transduction. The CICR contributes to the $\mathrm{Ca}$ transient responsible for mechanical activity, but also generates $\mathrm{Ca}$ waves propagating within the cytosol. We investigated subcellular Ca signals in neonatal rat and adult guinea-pig ventricular myocytes using ratiometric confocal microscopy with a mixture of two fluorescent $\mathrm{Ca}$ indicators. Individual resting myocytes exhibited spontaneous $\mathrm{Ca}$ release events from the sarcoplasmic reticulum (SR) that were attributable to three distinct patterns: i) local Ca release events without propagation; ii) planar $\mathrm{Ca}$ waves propagating across the cell; iii) spiral $\mathrm{Ca}$ waves spinning around a nucleus or shifting along a subcellular region without entering it. Transitions between these patterns were frequently observed, suggesting that a particular release pattern is not the property of an individual cell but reflects the functional state of the SR. The existence of focal release and refractory zones within a single cell indicates that the SR is not uniform on the subcellular level. The SR seems to be a network of elements with distinct functional properties. A subcellular variability in the positive feedback of the CICR mechanism can account for the observed features of $\mathrm{Ca}$ signaling. The degree of positive feedback exhibited by a single SR element may depend on its Ca content.

\section{S05-30}

IN VITRO EXPRESSION OF THE PARATHYROID HORMONE RECEPTOR IN RENAL EPITHELIAL CELL LINES

Hayes, G., Forgo, J., Biber, J. and Murer, $H$.

Physiology Inst., Univ. Zuerich.

To characterise the intracellular signalling and regulatory properties of the cloned parathyroid hormone (PTH) receptor we have stably transfected two renal epithelial cell lines with cDNA's encoding the PTH receptor (provided by $\mathrm{Dr}$ G. Segre). The proximal tubular, porcine (LLC PK1 [clone 4]), and the distal tubular, Madine Darby canine (MDCK) cell lines were transfected since the culture of either cell line on permeant filter supports leads to the development of polarised epithelial cell layers that mimic the epithelial organisation in vivo. Transfection of the cloned PTH receptor into each of these cell lines allowed us to examine, independently, the functional properties of the receptors expressed at the apical and/or the basolateral cell surface. While the basolateral application of PTH produces a large increase in cAMP production in both cell lines, the relative efficacy of an apical application of PTH differs between the cell types. The transfected LLC PK 1 cells exhibit a greater response to apical PTH compared to the transfected MDCK cells. PTH-mediated regulation of intrinsic phosphate transport was also examined in polarised, transfected MDCK cells. Neither apical or basolateral application of PTH affects intrinsic apical or basolateral $\mathrm{Na}$-dependent or phosphate transport activities. Similar experiments are being performed with the transfected LLC PKI cells. 
STRUCTURE- FUNCTION- ANALYSIS OF HATRLESS, A GENE INVOLVED IN DROSOPHILA NERVOUS SYSTEM DEVELOPMENT Maier, D., Thompson- Fontaine, A. and Preiss, A. Biozentrum der Universitat Basel, CH 4056 Basel

Hairless, a recessive larval lethal mutation, causes dominant defects in sensory organs of adult Drosophila flies. Manifold genetic interactions indicate that Hairless antagonizes neurogenic gene function suggesting an involvment also in neurogenesis. In order to gain insight into its function we have cloned the Hairless gene which encodes an extremely basic, very serine rich protein of $110 \mathrm{kD}$ calculated molecular weight. No significant homologies to proteins of known function could be identified. Therefore, we are concentrating by various means on a structure - function analysis of the Hairless protein. Firstly, we are testing a series of Hairless deletion constructs for rescue capacity and generation of anti-Hairless phenotypes after overexpression compared with the wild type gene. SecondIy, we are analysing HAIRLESs protein distribution throughout development, also at the subcellular level. Heat induced HAIRLESs protein runs at $\geq 150$ $\mathrm{kD}$, possibly due to phosphorylation which might be intrinsic to Hairless function, a hypothesis we are currently scrutinizing.

\section{S05-32}

FUNCTIONAL COUPLING OF PPARS AND TRS

IJpenberg, A., Wahli, W. , Desvergne, B., Institut de Biologie Animale, 1015 Lausanne

We are interested in a possible functional coupling of thyroid hormone (TR) and peroxisome proliferator-activated receptors (PPAR). To this end, we performed cotransfection experiments in NIH $3 \mathrm{~T} 3$ celis using CAT reporter constructs containing the promoter of either the T3 responsive malic enzyme (ME) gene, or the PPAR regulated acyl CoA oxidase (Aco) gene.

The ME reporter construct showed very moderate response to the activated mouse PPAR (mPPAR), whereas combination of $T R \alpha 1$ and unactivated mPPAR potentialized the $\mathrm{T} 3$ induction. A putative PPAR response element was identified within this promoter and will be further investigated.

The Aco reporter constructs were not responsive to TRs. In constrast, $\mathrm{A} T R$ and $T 3$ dependent inhibition of the PPAR mediated activation was observed.

The mechanism of functional coupling of these receptors, which may possibly involve the 9-cisretinoic acid receptor RXR, will be further investigated by molecular analysis.

\section{S05-33}

\section{ID EXPRESSION DURING XENOPUS DEVELOPMENT}

Zhang, H.,Reynaud, S. and Spohr,G.

Departement de Biologie Cellulaire, Sciences III,

30, quai Ernest-Ansermet, $\mathrm{CH}-1211$ Genève 4

Id cDNAs expressed during early Xenopus development have been isolated and sequenced. The encoded protein is homologous to the proteins described in higher vertebrates. Northern blot analysis reveals that transcription starts soon after mid blastula and decreases to some extent after tailbud-stage. Lower levels of transcription are detected also in adult frog tissues such as liver and heart.

Microinjection into oocytes of in vitro-synthesized MyoDor/and Id- mANA, along with a CAT reporter gene whose expression is under the controll of an $\alpha 3$-actin promoter supplemented with four E-boxes shows that Myo $D$ function is repressed by ld.

To investigate the importance of negative regulatory sequences we have isolated and characterised the Id promoter. As a strategy for identifying cis-regulatory elements, chimeric genes consisting of different 5 ' elements of the promoter were attached to the CAT coding sequences and microinjected into embryos.
Thy-1, CD26, CD45, p56/ck, AND p59fyn FORM MULTIMERIC COMPLEXES IN THE MEMBRANE OF A T LYMPHOMA CELL.

S. Arni, G. Senaldi, M. Poincelet, M. Pierres* and D.C. Hoessii. Department of Pathology, Centre Médical Universitaire, Geneva, Switzerland and Centre d'Immunologie de Marseille-Luminy*, Marseille, France.

Thy-1 and CD26 surface glycoproteins are both capable of delivering proliferative signals to $T$ cells upon cross-linking with appropriate antibodies. The tyrosine phosphatase $\mathrm{CD} 45$ is a key regulator of $T$ cell proliferation as it controls the activity of src family kinases p56/ck and p59fyn. Associations of Thy- 1 with CD45, and of $C D 26$ with $\mathrm{CD} 45$ have been reported after chemical cross-linking of intact cells and it is likely that such associations constitute part of the structural basis for the T-cell stimulating capacity of Thy- 1 and CD26 accessory molecules.

We report that Thy-1, CD26 and CD45 can be specifically crosslinked at the cell-surface and isolated as multimolecular complexes containing a $78 \mathrm{kDa}$ protein that is recognized by an anti-BiP (Grp78) antibody. In vitro kinase assays show the selective association of p56/ck and p59fyn with Thy-1 and CD45 contained in multimolecular complexes.

The multimolecular structure we describe could representa transducing unit incorporating surface receptors and regulators of tyrosine phosphorylation that are stabilized through interaction with the BiP protein in the plasma membrane.

S05-35

HSP90 IN YEAST: ITS ROLE IN SURVIVAL AND STEROID RECEPTOR REGULATION

Louvion, J.-F., Palmer, G. and Picard, D.

Département de Biologie Cellulaire, Université de Genève, $\mathrm{CH}-1211$ Genève 4

Unliganded steroid receptors form a complex with HSP90 via their hormone binding domain (HBD). The receptor activation involves a hormone-induced release of HSP90.

We genetically addressed the role of HSP9O in budding yeast by analyzing three kinds of HSP82 (the essential yeast homologue of HSP90) mutants. These mutations affect either the quantity of the protein (low versus normal levels), its integrity (deletion analysis) or its nature (HSP82 homologues from other species). HSP82 mutant are examined for their ability to complement a HSP82 deletion mutant. Moreover, they are tested for functional interaction with a HBD. Interestingly, a viable deletion of a charged region, suspected to be involved in HSP82-HBD interaction, only slightly interferes with hormonal activation of both estrogen or glucocorticoid receptors Furthermore, a short deletion in the last third of HSP82 leads to the production of a dominant negative mutant which prevents cell growth at elevated temperature. The ability of various HSP 82 homologues to palliate or not the absence of the yeast protein allows us to define. pallate or not the absence of the yeast protein allows us to define,
using chimeras, the regions of the protein which are necessary and sufficient to support cell life.

\section{S05-36}

DIFEERENTIAL TR $\alpha 1 ＼mathrm{~ Ｔ R ß 1 ~ T R A N S A C T I V A T I O N ~ A N D ~}$ TARGET PROMOTER CONTEXT

E. Jeannin and $B$. Desvergne. Institut de Biologie Animale, 1015 Lausanne.

The thyroid hormone $\mathrm{T} 3$ regulates gene expression through nuclear T3 receptors (TRS). Two distinct genes, $\alpha$ and $\beta$, encode three TR subtypes that have a certain tissue specificity and are differently expressed during development. Two T3 responsive genes, the myelin basic protein (MBP) and the malic enzyme (ME) genes are preferentially regulated by TRßI and $T R \alpha 1$, respectively. Our present work demonstrates that within the promoter of these genes, three classes of elements are involved in this regulation: 1) the T3 response element (TRE) organized as as an inverted palindrome (in MBP) or a direct repeat (in ME) 2) the transcription initiation site, GCrich or TATA-containing in $\mathrm{ME}$ and $\mathrm{MBP}$, respectively 3) binding sites for transcription factors such as SP1 or AP1. The preferential TR $\beta 1$ activation requires the presence of both the TRE of $\mathrm{MBP}$ and a TATA-containing initiation site. However, transcription factors binding sites placed between these two elements can overcome the TRB1 preference. 
S05-37

TISSUE DISTRIBUTION AND DEVELORMENTAL EXPRESSION OF THE RAT PPARQ.

T.Lemberger, O.Braissant, and W.Wahli, Institut de Biologie Animale, 1015-Lausanne.

We are studying the tissue distribution of the rat Peroxisome Proliferators Activated Receptor $\alpha$ (PPAR ), a member of the nuclear hormone receptors superfamily, during development and in adults.

High levels of PPAR $\alpha$ mRNAs are detected in adult liver, kidney and heart. Muscle, stomach, and intestine show moderate amounts of the PPARa, whereas brain, testis and lung present a very low expression. During postnatal development, we observe a continuous increase of the PFARn expression in the kidney, in contrast to a steady level in the liver.

Histological distribution of PFARa transcripts is determined by in situ hybridization, both on adult rats and embryonic stages. The localization of the PRAR in well defined anatomical structures will hopefully give us clues to its physiological roles.

505-38

DNA BINDING PROPERTIES AND STIMULATION OF GENE TRANSCRIPTION OF BACULOVIRUS EXPRESSED XPPAR $\beta$.

Hihi, A.K, Mermod, N., Medin, J., ozato, K. and Wahli, w., Inst. de Biol. Animale, Úni Lausanne, $\mathrm{CH}-$ 1015 Lausanne. Lab. of Mol. Growth Reg., NIH, Bethesda, MD, 20892 USA.

The peroxisome proliferators activated receptors (PPARs) are members of the steroid/thyroid nuclear receptor superfamily. So far, they have been found in amphibians, rodents and humans. In Xenopus laevis, 3 subtypes (XPPAR $\alpha, \beta, y$ ) have been isolated. In order to study the mode of activity of the $\beta$ form, the xPPARB gene product was overexpressed using a baculovirus expression system. The nuclear protein obtained has the correct molecular weight of $46 \mathrm{KD}$. Gel shift assays showed that nuclear extracts containing the recombinant protein are able to specifically bind the 'PPAR response element' which is a direct repeat of the core AGGTCA motif. This DNA binding activity is potentiated by another nuclear receptor, the mouse RXR $\beta$ and is inhibited by phosphatase, suggesting a role for phosphorylation in EPAR binding to DNA. A functional approach, using an in vitro transcription system, was chosen to define PPAR and RXR action on transcription stimulation, as well as the role of their respective activators.

\section{S05-39}

\section{PROMOTER ANALYSES OF A GROWTH FACTOR REGULATED GENE}

T. Trüb, M. Kalousek, R. Kessler, and R. Klemenz

Dept. of Pathology, University Hospital, 8091 Zürich

Stimulation of quiescent cells to enter the cell cycle results in altered gene expression. Some of the first genes to be induced (immediate early (i.e.) genes) encode transeription factors which are thought to pass on the mitotic signal to the delayed early (d.e.) genes. We have analysed the promoter elements required for growth factor mediated induction of the d.e. mouse gene $\mathrm{T} 1$ which encodes a secreted glycoprotein of the immunoglobulin superfamily. A $448 \mathrm{bp}$ region located between 3.6 and $4.0 \mathrm{~kb}$ upstream of the transcription start site is sufficient for growth factor mediated inducibility. It contains an AP-1 binding site which is absolutely required for $T 1$ gene expression. It is surrounded by 3 E-boxes. At least 2 of them are essential for efficient gene induction. Thus the i.e. proteins encoded by the fos and jun gene family which form the AP-1 complex can activate the d.e. gene $\mathrm{T} 1$ in collaboration with helixloop-helix transcription factors binding to the E-boxes.
SO5-40

AN UNUSUAL CAMP DEPENDENT RROTEIN KINASE IN
Dictyostelium discoideum

Anjard, C., Groux, D., Gamboni, S. and Reymond, C.D. Institut d'histologie, UNII, Rue du Bugnon 9. 1005 I.AUSANNE.

The cAMP dependent protein kinase (cAPK) from Dictyostelium is composed of a single catalytic (C) and a single regulatory (R) subunit. The $C$ subunit we characterised, PkaC is about twice the size of its mammalian counterpart. We showed that it possesses all properties of a catalytic subunit. It associates with the $R$ subunit in absence of cAMP, it copurifies with cAPK activity and an increased activity is observed in cells over-expressing PkaC. Eurthermore, we dissected its role during development and cell differentiation. Overexpressing pkaC undex cell type specific promoters allowed to show the requirement for its activity during spore differentiation. PkaC seems to play a more complex role in prestalk cells.

We are now dissecting the functional regions of the PkaC protein, making use of the known tertiary structure of the $C$ subunits of mammalian enzymes.

S05-41

\section{ARACHIDONIC ACID-INDUCED Ca $2+_{i}$ OSCILLATIONS IN SMOOTH MUSCLE CELLS FROM HUMAN AIRWAYS: EFFECTS OF CYCLOOXYGENASE AND LIPOXY- GENASE INHIBITION. \\ J. Durand and N. Marmy \\ Institut de Physiologie, 5 rue du Musée, 1700 Fribourg}

Arachidonic acid $(0.5-20 \mu \mathrm{M})$ induces various patterns of $\mathrm{Ca}^{2+}{ }_{i}$ signal in bronchial smooth muscle cells, as revealed by single cell dynamic video imaging of Fura-2 loaded cells. Most frequently, $\mathrm{Ca}^{2+}$ oscillations were observed, although other patterns (a single $\mathrm{Ca}^{2+}{ }_{\mathrm{i}}$ transient; a single peak followed by a sustained elevated level, or a sustained $\mathrm{Ca}^{2+}{ }_{i}$ elevation solely) were occasionally seen. The amplitude of $\mathrm{Ca}^{2+}{ }_{i}$ oscillations was related to the concentration of arachidonic acid. The frequency histogramm was noticeably shifted to higher frequencies by nordihydroguaiaretic acid (NDGA, $0.2 \mu \mathrm{M}$, a lipoxygenase inhibitor), whereas it was markedly shifted to lower frequencies by indomethacin $(50 \mu \mathrm{M}$, a cyclooxygenase inhibitor) and by $5,8,11,14$-eicosatetraynoic acid (ETYA, $3 \mu \mathrm{M}$, a lipoxygenase and cyclooxygenase inhibitor). These observations suggest that: (1) arachidonic acid per se elicits $\mathrm{Ca}^{2+}{ }_{\mathrm{i}}$ oscillations in these cells; (2) cyclooxygenase activity products modulate the frequency of these oscillations.

\section{S05-42}

PLC ACTIVITY IN HUMAN AIRWAY SMOOTH MUSCLE CELLS: MODULATION BY CALCIUM, PKC AND PLA2 N. Marmy and J. Durand

Institut de Physiologie, 5 rue du Musée, 1700 Fribourg

The increase of radiolabelled inositol phosphates ([ $\left.\left.{ }^{3} \mathrm{H}\right] \mathrm{IPs}\right)$ production in agonist-stimulated human airway smooth muscle cells (ASMC) was determined by HPLC. In order to observe the role of calcium, PKC and $\mathrm{PLA}_{2}$ on PLC activity during agonist stimulation, the effects of pharmacological agents (able to alter calcium, PKC and PLA 2 ) were tested on IP production elicited by a $5 \mathrm{sec}$ stimulation with histamine. The inhibitor of PKC staurosporine increased, while PMA (an activator) decreased the histamine-stimulated production of $\left[{ }^{3} \mathrm{H}\right] 1,4,5-\mathrm{IP} 3$ and of its degradation products. An inhibition was also observed with the two $\mathrm{PLA}_{2}$ inhibitors, quinacrine and $p$-bromophenacyl bromide. In calciumfree buffer, no difference in the IP increase was shown, but an inhibition was observed when thapsigargin, an inhibitor of the $\mathrm{Ca}^{2+}, \mathrm{Mg}^{2+}$ ATPase of the sarcoplasmic reticulum, was added in the same conditions. The calcium ionophore ionomycin increased the IP production in presence of external calcium, whereas it completely blocked the stimulation in calcium-free buffer. The results suggest that PLC activity, in human ASMC, is modulated by a positive feedback of calcium and by a negative feedback of PKC and PLA2 
$505-43$

REGULATION OF HORMONE-STIMULATED CALCIUM SIGNALS

M. Bootman, AFRC, Laboratory of Molecular Signalling, Dept. Zoology, Cambridge, UK Stimulation of cells with hormones that activate inositol 1,4,5-trisphosphate $\left(\mathrm{InSP}_{3}\right)$ formation, leads to an increase in the intracellular $\mathrm{Ca}^{2+}$ concentration $\left(\left[\mathrm{Ca}^{2+}\right]\right.$ ). These hormone-stimulated $\left[\mathrm{Ca}^{2+}\right]_{i}$ signals have a complex temporal and spatial regulation, with the $\left[\mathrm{Ca}^{2+}\right]_{i}$ increase often taking the form of a series of repetitive spikes or oscillations, arising from a steady basal level. The spatial counterpart of a $\left[\mathrm{Ca}^{2+}\right]$ spike is a wave, where $\left[\mathrm{Ca}^{2+}\right]$, is first elevated in a localised region of a cell, and then spreads throughout the cell in a regenerative manner. The $\left[\mathrm{Ca}^{2+}\right]_{i}$ spike frequency is modulated by several environmental factors including hormone concentration, extracellular calcium and thiol reagents. Current evidence suggests that a cell can interpret these complex $\left[\mathrm{Ca}^{2+}\right]$, changes to regulate a diverse range of cellular activities. Although many of the biochemical steps between hormonereceptor activation and the $\left[\mathrm{Ca}^{2+}\right]_{i}$ response are known, the precise mechanism generating these complex $\left[\mathrm{Ca}^{2+}\right]_{\mathrm{i}}$ signals is unclear. In order to understand this mechanism more clearly, we have investigated the regulation of Ins $\mathrm{P}_{3}$-mediated $\mathrm{Ca}^{2+}$ release from intracellular stores in intact cells. Our current data suggests that under certain conditions, the $\mathrm{InsP}_{3}$-sensitive intracellular stores behave as functionally discrete units, and that during a $\mathrm{Ca}^{2+}$ spike these stores become functionally coupled by the diffusion of $\mathrm{Ca}^{2+}$ from one store to the next, triggering a regenerative $\mathrm{Ca}^{2+}$ release.

\section{S05-44}

CHARACTERIZATION OF THE DROSOPHILA HOMOLOG OF RAC PROTEIN KINASE

Andjelković, M. , Cron, P., Grossniklaus, U*. and Hemmings, B.A.

Friedrich Miescher-Institut, PO Box 2543, $\mathrm{CH}-4002$ Basel and *Biozentrum der Universitât Basel, Klingelbergstrasse 70, $\mathrm{CH}-4056 \mathrm{Basel}$.

RAC-PKs (Related to PKA and PKC protein kinases) represent a serine/threonine kinase family whose catalytic domain is closely related to those of protein kinases $A$ and $C$. They contain $a \mathrm{PH}$ (pleckstrin homology) domain $\mathrm{N}$-terminal to the catalytic domain. In addition, RAC-PK $\alpha$ was shown to be a proto-oncogene. In order to investigate the role of $\mathrm{RAC}-\mathrm{PK}$ in cellular signalling we undertook genetic and biochemical analysis of the Drosophila homolog (DRAC-PK). The DRAC-PK gene encodes multiple classes of transcripts. Antisera raised against the DRAC-PK recombinant protein specifically recognize 3 distinct molecular weight forms $(68,85$ and $120 \mathrm{kDa})$. All forms are expressed throughout development and are both maternally and zygotically regulated. The DRAC-PK gene is localized at 89B4-10 region of the third chromosome, between the pannier and the stubble genes.

\section{S05-45}

FUNCTIONAL ANALYSIS OF THE HUMAN IFN-INDUCIBLE

\author{
J.F.Goetschy ${ }^{1}$, J.DE GENE PROMOTRR.
J.Dellig \\ M.A.Horisberger 1

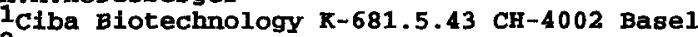 \\ 2 Institut Pasteur du Brabant, B-1080 Bruxelles
}

The human $\mathrm{ExA}$ promoter has two Interferon Stimulated Responsive klements (ISRE) at positions $-47 /-56$ and $-94 /-103$, and a Sp1 binding $81-$ te. We have compared the functionality of the promoter region $-525 /+8$ and of the promoter region $-525 /+1061$ comprising the $1^{\text {st }}$ intron and the $2^{\text {nd }}$ exon. The reporter gene was the soluble receptor for IgE which has been measured in the culture medium of stably transfected cell lines (human L132 and hamster CHO cell lines). Both promoters were IFN-inducible at the transcriptional level. Differences in the level of protein expression could be related to transcriptional regulation and to an effect of the presence of the exon/Intron at the translational level. In human cells the endogenous natural MXCA promoter was much atronger (4-20 fold) than the MxA promoter introduced by the plasmid. A base deletion at position -498 designed to restore an ISRE consensus sequence completely silenced both promoters.
S05-46

Phenylarsenoxide as a tool for identifying proteins involved in the secretory process

Wiedemann, C, Schaefer, T., Gitler, C., Burger, M. M. Fricdrich-Miescher Institut, P.O.Box 2543, CH-4002 Basel Department of Membrane Research and Biophysics, Weizmann Institute of Science, Rehovot 76110, Israel

Phenylarsenoxide (PAO) at $20 \mu \mathrm{M}$ blocks exocytosis in chromaffin cells of the bovine adrenal medulla. This inhibition can be reversed by small dithiols, such as dithiothreitol or 2,3-dimercaptopropanol. Because trivalent arsenicals interact specifically with dithiol-containing proteins, we conclude that dithiol-proteins do play an important role in regulated sectetion in chromaffin cells.

To identify dithiol-proteins putatively involved in the secretory process, we compare PAO-binding proteins from chromaffin and $\mathrm{PC1} 2$ cells and from fibroblasts as well as from subcellular fractions by 2-dimensional gelelectrophoresis. The proteins are identified by radioactive PAO bound to the dithiol in a complex that withstands SDS-PAGE.

Affinity chromatography on PAO-Sepharose with various protein fractions is used to purify the dithiol-proteins of interest Enzymatic activity, e.g. kinase or phosphatase activity, can be measured in the cluates of such a column to give further hints at the possible function of dithiol-proteins.

\section{S05-47}

GLUTATHIONE IMPEDES THE ACCUMULATION OF HYDROPEROXY ARACHIDONATE METABOLITES IN CHICK SPINAL CORD MENINGES.

M.F. Vesin and C. Billotte, Institut d'Histologie et d'Embryologie, Université de Lausanne, rue du Bugnon 9, 1005 Lausanne

When spinal meninges homogenates were incubated with reduced glutathione (GSH), a cofactor of prostaglandin (PG) biosynthesis, $\left[{ }^{14} \mathrm{C}\right]$ arachidonate (AA) was bioconverted into a major and a minor product which comigrated on TLC with $\mathrm{PGE}_{2}$ and $\mathrm{PGD}_{2}$ respectively. In absence of GSH: 1) $\mathrm{PGD}_{2}$ could not be detected; 2) the level of $\mathrm{PGE}_{2}$ was dramatically reduced but surprisingly counterbalanced by accumulation of an unusual $\left[{ }^{14} \mathrm{C}\right] \mathrm{AA}$ metabolite which exhibits particular traits of migration on TLC and of retention time on HPLC. This AA metabolite: 1 ) does not correspond to a degradation product of $\mathrm{PGE}_{2}$; 2) crossreacts with $\mathrm{PGE}_{2}$ antibody; 3) fits the conditions required for a 15 hydroperoxy $\mathrm{PGE}_{2}$ (chemical degradation into $\mathrm{PGE}_{2}$ by hydroperoxyde reducing reagents such as $\mathrm{SnCl}_{2}$ or GSH-hemin).

It is therefore inferred that depletion of GSH favours accumulation of hydroperoxyprostaglandins which would participate to oxidative injury.

\section{S05-48}

DNA-BINDING STUDIES USING BACTERIALLY EXPRESSED ECDYSTEROID RECEPTOR AND ULTRASPIRACLE FROM CHIRONOMUS TENTANS AND DROSOPHILA MELANOGASTER

Carsten Elke, Martin Vögtli and Markus Lezzi Institut für Zellbiologie, ETH Hönggerberg, CH-8093 Zürìch

The ecdysteroid receptor (EcR) and ultraspiracle (USP) from both, Chironomus tentans and Drosophila melanogaster, were expressed in $E$. coli as fusion proteins with glutathione-S-transferase. The identity of the expressed recombinant proteins was confirmed by Western blot anal-

The Drosophila EcR binds to its cognate hormone response element as a The Drosophila EcR binds to its cognate hormone response element as a bilities of the two receptor partners from both insect species in regard to DNA binding and heterodimerization by means of gel mobility shift assays. The use of naturally occurring and artificial hormone response elements will allow to define a hormone response element consensus sequence for EcR-USP heterodimers and, if existing, for EcR and USP homodimers, respectively.

For other members of this receptor family it was shown that the DNA binding domain and adjacent sequences alone sufficiently defines response element specificity for both homo- and heterodimers. The DNA binding domains of ECR and USP from the two species show a high similarity ( $92 \%$ and $85 \%$ identity, respectively). The corresponding sequences were selected for overexpression in $E$. coli. In combination with immunoprecipitation of receptor-DNA complexes and PCR amplification steps, the expressed proteins will be used for the detection of naturally occuring hormone response clements within genomic DNA. 
S05-49

PDGF-INDUCED PHOSPHATIDYLINOSITOL 3-KINASE ACTIVATION MEDIATES ACTIN REARRANGMMENTS IN FIBROBLASTS

M. 2. Wymann \& A. Arcaro, Institute of Biochemistry, University of Fribourg, $\mathrm{CH}-1700$ Fribourg

various agonist-induced cell responses in neutrophils and fibroblasts, such as chemotaxis and cytoskeletal rearrangements, have been shown to parallel the synthesis of ptdins $(3,4,5) P_{3}$. But the importance of this rise is not clear. We show here that wortmannin blocks PDGF-induced production of ptdins $(3,4,5) P_{3}$ in human foreskin fibroblasts with an IC 50 of about $5 \mathrm{nM}$. A similar inhibition was observed in in vitro assays (IC50 $=1 \mathrm{nM}$ ) with PI 3 -kinase immunoprecipitated by antibodies directed against the $85 \mathrm{kD}$ subunit (p85). On the other hand, wortmannin did not affect PDGF-mediated phosphorylation of p85, indicating the correct interaction of $\mathrm{p} 85$ with the PDGF- $\beta$ receptor. The p110/p85 complex of the PI 3-kinase remained intact in the presence of $\mu \mathrm{M}$ concentrations of wortmannin. These results are consistent with a direct, specific inhibition of PI 3kinase by wortmannin at concentrations relevant for its previously reported effects on cellular responses. When stimulated with PDGF, human foreskin fibroblasts form circular membrane ruffles rich in filamentous actin. The fact that wortmannin inhibits these PDGF-mediated actin rearrangements suggests the need of PI 3-kinase activity as a signal for this cell response.

\section{S05-50}

\section{A ROLE FOR PIP3 IN THE NEUTRORHIL RESPIRATORY} BURST AND CYTOSKELETON

Arcaro A. and Wymann M.P., Institute of Biochemistry. University of Fribourg, CH-1700 FRIBOURG

Chemoattractants like N-formyl-Met-Leu-Phe (fMLP) stimulate in neutrophils a rapid and transient increase in the levels of Ptdins $(3,4,5) \mathrm{P}_{3}$ ( $\left(\mathrm{PI}_{3}\right)$ which is due to the activation of PtdIns (3)-kinase. PIP 3 has been proposed to be a second messenger molecule, but its cellular targets are presently unknown. Here we report that pretreatment of human neutrophils with wortmannin inhibits the fMLP-stimulated production of $\mathrm{PIP}_{3}$ in a dose-dependent manner ( $\mathrm{IC}_{50}=5 \mathrm{nM}$ ). Furthermore, ptdins(3)-kinase activity immunoprecipitated from resting cells is totally abolished by $10 \mathrm{nM}$ wortmannin (IC50 $=1 \mathrm{nM}$ ). These results show a potent and direct effect of wortmannin on PtdIns (3)-kinase. Wortmannin also inhibits the respiratory burst of neutrophils at nanomolar concentrations, which suggests that $P I P 3$ is involved in the signaliing pathway controlling activation of the NADPHoxidase. When pretreated with wortmannin and stimulated with fMLP, neutrophils display oscillatory changes in Factin content that correlate with ascillations in cell shape. This, and the inhibition of PtdIns(3)-kinase, suggest a modulatory role for $\mathrm{PIP}_{3}$ in cytoskeletal rearrangements. We are currently investigating the effects of PIP 3 on the functions of geisolin, a protein that is proposed to mediate actin polymerization and can be zegulated by polyphosphoinositides and $\mathrm{Ca}^{2+}$.

S05-51

\section{ULTRASPIRACLE (USP) MEDIATES ENHANCED NUCLEAR TRANSLOCATION OF THE ECDYSTEROID RECEPTOR (ECR) IN VERTEBRA'TE CELLS}

Martin Vögtli, Markus O. Imhof, Sandro Rusconi*, and Markus Lezzi, Institute for Cell Biology, Swiss Federal Institute of Technology, Zurich, and *Institute for Molecular Biology II, University Zurich-Irchel, Zurich.

In general, untransformed nuclear hormone receptors are predominantly confined to either the nucleus or the cytoplasm of a cell. Receptors of the latter class are translocated to the nucleus upon interaction with ligand. Our studies of the use of the ecdysteroid receptor complex from insects, consisting of a heterodimer formed between EcR and USP, as a tool for target gene activation in vertebrate cells have revealed a novel mechanism of nuclear translocation of EcR that does not depend on hormone action. After transient transfection of respective expression plasmids into HeLa or CV-1 cells, the subcellular distribution of the individual receptor types was analyzed by indirect immunofluorescence staining. While EcR alone was detected in both cellular compartments, nucleus and cytoplasm, USP was predominantly found in the nucleus of either host cell. Coexpression of both EcR and USP resulted in an efficient translocation of $E c R$ into the nucleus. USP appears to trap EcR in the nucleus, presumably by the formation of a heterodimeric complex. Thus, heterodimer formation of EcR and USP not only appears to be required for high affinity binding to cognate hormone response elements (and subsequently for transactivation of an adjacent target gene) and for binding to ecdysteroid but in addition for an enhanced translocation of EcR into the nucleus.
S05-52

\section{DISTRIBUTION OF THE CALCINEURIN IN RAT TISSUE}

Su, Q.; Weber, L.; Zenke, G. $\$$ and Ryffel, B. Institut of Toxicology, ETH and University of Zürich; §Sandoz Pharma Ltd. Basel, Switzerland

Calcineurin is a calcium/calmodulin-regulated protein phosphatase which is comprised of a catalytic subunit $A$ and regulatory subunit $B$. Although calcineurin was discovered in brain, the calmodulin stimulated protein phosphatase which has calcineurin like structure has been described in other tissues. The protein structure show that the calcineurin $B$ is very conserved in different tissues and species. Using anti-calcineurin $B$ monocional antiserum, the tissue extracts from rat have been explored. Immunoreactivity was obverved in all tissues, although its intensity was different. The highest intensity was found in brain. Spleen, Heart and thymus had an intermediate level intensity. The results were supported by the quantitative measurement of calcineurin. The calcineurin concentration was 10 to 50 fold higher in brain than that in other tissues. Furthermore, the distribution of the calcineurin activity in rat tissue was studied using dephosphorylation assay of calcineurin. The highest calcineurin activity was found in brain too, but was only 5 to 15 fold more than that in other tissues. The specific activity of calcineurin was different in tissues. These results indicate the calcineurin activity might be regulated in tissue specific fashion.

\section{S05-53}

\section{ISOLATION OF OCT-2A INTERACTING PROTEINS UTILISING THE YEAST TWO HYBRID SYSTEM}

Matthias Gstaiger, Walter Schaffner and Christopher Hovens Institut für Molekularbiologie II der Universität Zürich, CH 8057 Zürich

The octamer motif ATGCAAAT plays a central role in mediating the activity of a number of both lymphoid specific and ubiquitous promoters and in the immunoglubulin heavy chain intronic enhancer. The activity of thils motif in lymphoid cells is presumably mediated by the lymphoid cellspecific factor Oct-2A. When ectopically expressed in non-lymphoid cells, Oct-2A is not sufficient to mediate enhancer activity of a multimerized 50 bp enhancer core element derived from the immunoglobulin heavy chain intronic enhancer, a segment, which is however highly active in B-cells. This and other findings support the supposition that additional B-cell specific factor(s) can account for the specificity and extent of the Octdependent transcription observed in lymphoid cells. To further our understanding of the molecular mechanisms involved in mediating lymphoid-specific expression, we have employed the yeast two hybrid system to identify human proteins capable of physically associating with human Oct-2A. To this end, a Oct-2A expressing yeast strain has been constructed which bears two integrated reporter genes, HIS3 and lacZ under the control of the octamer motif. Transformation of this strain with a cDNA library has allowed us to isolate clones interacting with Oct-2A. We are currently analysing these candidates to determine the veracity of these interactions in higher eukaryotic cell background.

\section{S05-54}

BACTERIA INDUCE L-ARGININE-DEPENDENT NITRIC OXIDE SYNTHESIS IN BOVINE MACROPHAGES.

H. Adler, E. Peterhans, and T.W. Jungi

Institute of Veterinary Virology, University of Berne, Berne, Switzerland

Activation of rodent macrophages with cytokines or bacteria is accompanied by the induction of a $\mathrm{Ca}^{2+}$-independent nitric oxide (NO) synthase which plays a key role in antimicrobial and antitumoral activity. Firm evidence for expression of a similar enzyme in other mammals or in man has been lacking. We now show that bovine bone marrow-derived macrophages produce nitrite in an I-arginine-dependent manner upon stimulation with heat-killed grampositive or gram-negative bacteria. Homologous interferon- $\gamma$ and tumor necrosis factor- $\alpha$ induced little $\mathrm{NO}_{2}^{-}$production, but primed macrophages for enhanced $\mathrm{NO}_{2}{ }^{-}$production induced by Salmonella dublin or LPS. This is one of the first demonstrations of $\mathrm{NO}_{2}{ }^{-}$production by nonrodent macrophages and encourages a search for an involvement of reactive nitrogen species in the killing of parasites or tumor cells by macrophages from mammals other than rodents. 
P75NGFR MODULATES TRK KINASE ACTIVITY, BUT NOT LIGAND INTERNALIZATION

P. Kahle, P. Barker*, A. Trzeciak, G. Weskamp*, E. Shooter", and C. Hertel

Hoffmann-La Roche, Basel, "Stanford University School of Medicine, Stanford, California, *SloanKettering Institute, New York

The biological activity of NGF is thought to be mediated by the Trk receptor tyrosine kinase. The role of the apparently not signal-transducing p75NGER is less clear. We have investigated in PC12 cells the relative contribution of Trk and p75NGFR for ligand internalization and tyrosine phosphorylation of Trk, Shc, PLC $\gamma_{-1}$, and ERKs. NGF internalization was neither prevented by inhibiting Trk kinase activity, nor by abolishing NGF binding to $75^{\text {NGFR }}$. However, when NGF binding to Trk was diminished, NGF internalization was decreased. Selective activation of Trk led to an more rapid and more prolonged kinetics of tyrosine phosphorylation. Thus, NGF internalization in PC12 cells apparently occurred independently of p75 NGFR. Trk binding, even without kinase activation, seemed to be sufficient for this process. The p $75^{\text {NGFR }}$ receptor rather seemed to have a modulatory role on NGF-induced Trk kinase activity.

\section{Translation in Eukaryotes}

S06-01

ANALYSIS OF GLYCOPROTEINS SEPARATED BY TWODIMENSIONAL GEL ELECTROPHORESIS USING LECTIN BLOTTIYG REVEALED BY CHEMILUMINESCENCE.

Gravel $P^{1}$, Golaz $O^{2}$, Walzer $C^{1}$, Hochstrasser $D F^{2}$, Turler $\mathrm{H}^{3}$ and Balant LP. 1

${ }^{1}$ Clinical Research Unit, Psychiatric University Institutions of Geneva 2 Univ.Cantonal Hospital of Geneva ${ }^{3}$ Dept. of Molecular Biology, Univ. of Geneva, Switzerland Lectins are used in many analytical methods to study the carbohydrate structure of glycoproteins. We report here a technique which combines the high resolution of two-dimensional gel electrophoresis (2-DPAGE) to separate plasma proteins, the specificity of lectins to bind carbohydrates and the sensitivity of chemiluminescence. This method is compared with that of a commonly used colorimetric reaction.

Since the reference plasma protein map obtained by 2-D PAGE is available (1), the technique described here allows a quick and specific identification of plasma glycoproteins. Therefore any major glycosylation modification which may happen in some diseases can be detected.

(1) Electrophoresis 1992; 13:707-714.

This work was supported in part by FCAR (Quebec, Canada).

\section{$506-02$}

TOBACCO TRANSLATION INITIATION FACTOR EIF-4A IS ENCODED BY A LARGE AND DIVERSE GENE FAMILY

Karl Brander, Therese Mandel, Isabelle Lutziger, George owttrim and Cris Kuhlemeier, Institute of plant Physiology, University of Berne, Altenbergrain 21, $\mathrm{CH}-3013$ Berne

Using heterologous probes we isolated cDNAs coding for translation initiation factor eIF-4A from tobacco. eIF-4A is encoded by a large multigene family of which at least nine members are expressed in leaves and most if not all other organs of the tobacco plant. While screening genomic libraries we found several additional genes. Two of them code for genes highly homologous with eIF-4A throughout the coding region, but with changes in the GKT, PTRELA and DEAD-box motifs. A third gene is expressed exclusively in the developing male gametophyte. This eIF-4A-related gene may have a role in the well-documented regulation of translation in pollen. In order to study the function of these genes we have begun altering their expression in transgenic tobacco plants.
S06-03

A genetic search for new eukaryotic translation initiation factors Buser Peter, Coppolecchia Raffaella, Daugeron Marie-Claire, Stotz Agathe and Linder Patrick

Dept. of Microbiology, Biozentrum, Klingelbergstr. 70, 4056 Basel

In our laboratory we use genetic and biochemical approaches to study translation initiation, in particular the initiation factor elF-4A in yeast. This factor from higher eukaryotic cells is known as an RNA dependent ATPase and functions as a RNA helicase together with another initiation factor, elF-4B. It belongs to a family of putative RNA helicases, the D-E-A-D proteins. To find new genes involved in translation initiation, suppressors of a temperature sensitive eIF-4A mutant were isolated. One of the suppressors (STMI) is a single copy gene which encodes a protein resembling the human translation initiation factor eIF-4B. Disruption of the gene is not lethal to the cell, but affects growth. Polysome analysis of strains with a deleted STMI gene have shown that this new gene is also involved in translation initiation. It carries six repeated sequences of 25 amino acids of unknown function and has - like the human eIF-4B - a RNA binding domain. The second suppressor (STM2) is also a novel gene. It encodes a large protein which shows no homology to other proteins present in the data libraries. It contains high portion of charged amino acids and is rich in glutamines and serines. Its function in translation initiation is currently under investigation.

\section{S06-04}

Sequence requirements for a non-AUG protein initiation

Ronald Boeck and Dan Kolakofsky Dept. of Genetics and Microbiology University of Geneva Medical School

Non-AUG initiation codons are still infrequent and were initially observed in animal viruses, but a number of cellular examples have now also been reported. Several years ago we reported the use of an ACG in the P/C mRNA of Sendai Virus (SEN) to initiate the non-structural $C^{\prime}$ protein. We recently described a $C^{\prime}$ protein in the Human Parainfluenza Virus Type 1 (hPIV1), which is initiated from a GUG. In both cases, the $C^{\prime}$ protein is an N-terminally elongated form of the $C$ protein which is initiated at the second $A U G$, in the +1 reading frame relative to the first AUG. This AUG is used to initiate the $P$ protein, an essential component of the viral RNA polymerase. Unlike the ACG in SEN, the GUG in hPIVI is clearly not a weak start codon since $C^{\prime}$ is the most abundant protein expressed from this mRNA both in vivo and in vitro.

It appears that not any upstream GUG in an optimal context will function as a start codon. For instance, the $P$ gene of hPIV 3 cannot express a $\mathrm{C}^{\prime}$ protein (the ORF is closed by stop codons) but it does contain a GUG in an optimal context upstream of the P protein AUG, which could encode a $P^{\prime}$ protein. Nevertheless, we have been unable to demonstrate that this GUG functions as a start codon. With the use of chimeric mRNAs between hPIV1 and 3 , we have shown that positions +5 and +6 (the first $G$ of the $G U G$ triplet being +1 ) are the major determinants of the efficiency of GUG as an initiaton codon for protein synthesis. We are currently investigating the possibility that these nucleotides may increase the initiation rate of weak non-AUGs such as the ACG of SEN. 


\section{S06-05}

Biological activity of the heterodimeric subunit SRPg/14 of the Signal Recognition Particle is retained in 2 fusion proteins

Fabrice Bovia \& Katharina Strub, Département de Blologie Cellulaire, Université de Genève, $\mathrm{CH}-1211$ Genève 4

The targeting of secretory proteins to the membrane of the endoplasmic reticulum is mediated by a cytoplasmic ribonucleoparticle, the signal recognition particle. It is composed of 6 proteins and 1 FNA molecule (SRP RNA). The 2 smallest proteins SRP9 and SRP14 are required for the translational control function of SRP. It effects a specific arrest in the biosynthesis of ER-targeted proteins. These 2 polypeptides bind to the biosynthesis of ER-targeted proteins. These 2 polypeptides bind to the SRP RNA exciusively as a heterodimer (SRP9/14). We have constructed single-chain variants of this dimer using a short peptide linker of 17 aa to connect the C-terminus of the $14 \mathrm{kD}$ subunit to the $\mathrm{N}$-terminus of the $9 \mathrm{kD}$ subunit (14-9) and vice versa $(9-14)$. We found that both proteins bind SRP RNA with a similar efficiency as the wild type protein. Glutaraldehyde cross-linking experiments and sedimentation anaiysis indicate that the 2 fusion proteins fold into a similar structure as SRP9/14 and bind SRP RNA as a monomer. Furthermore, they can functionally replace SRP $9 / 14$ in the elongation arrest and translocation assay. Since the 2 fusion proteins are circular permutations of each other, we can conclude from this results that $\mathrm{N}$ - and $\mathrm{C}$ - termini of both proteins have no essential role in folding. RNA-binding and in mediating their biological activity. Furthermore, the possibility to express an oligomeric protein as a single polypeptide facilitates the analysis of its functions as well as its structure.

\section{S06-06}

\section{HETEROGENEITY OF THE GERM-LINE AND SOMATIC RIBOSOMES IN ASCARIS LUMBRICOIDES.}

Vincent Bernard, Adrian Etter, Heinz Tobler and Fritz Müller. Institute of Zoology, University of Fribourg, 1700 Fribourg (Switzerland).

The nematode Ascaris lumbricoides undergoes chromatin diminution which causes a loss of DNA in all somatic cells. We have identified a ribosomal protein (rp) gene (coding for ALEP1 = Ascaris lumbricoides eliminated protein 1) which is located within the germ-line specific material. ALEP1 shows strong homologies with the ribosomal protein $\mathrm{S} 19(\mathrm{rpS19})$. The transcription of its gene takes place only in the germ-line. 2D-gel electrophoresis on germ-line and somatic purified ribosome fractions shows that the ALEP1 protein ( $\mathrm{pS} 19$ ) is present only in the germ-line ribosome.

Does the ALEP1 germ-line specific protein have a homologue in the somatic ribosomes? We have cloned a homologue of ALEP1 from Ascaris somatic tissue named rpS19'. We are currently studying the expression pattern of this $\mathrm{rpS19}$. These results indicate the existence of a developmentally regulated ribosome heterogeneity between the germ-line and somatic cells.

What is the function of rpS19? Since Ascaris is not suitable for genetic analyses, we isolated $\mathrm{TpS} 19$ from the nematode Caenorhabditis elegans. The C. elegans rpS19 cDNA shows only $60 \%$ homology at the nucleic acid level. RpS19 is located on the C. elegans chromosome IV. The identification of a rpS19 mutant in C. elegans will indicate its function.

\section{S06-07}

TIF3, A NEW TRANSLATION INITIATION FROM SACCHAROMYCES CEREVISIAE WITH RNA HELICASE ACYIVITY

M. Altmann, P.P. Müller, B. Wittmer, F. Ruchti, S. Lanker and $H$. TrachseI

Institut für Biochemie und Molekularbiologie, Universităt Bern, Bühlstr. 28,3012-Bern, Schweiz.

Tif3 is a new eukaryotic initiation factor which shows 268 identity with the sequence of mammalian translation initiation factor eIF-4B. The TIF3 gene is not essential for growth; however, its disruption results in a slow growth and coldsensitive phenotype. In vitro translation of total yeast RNA in an extract from a TIF 3 gene-disrupted strain is reduced as compared to a wild-type extract. The translational defect is more pronounced at lower temperatures and can be corrected by the addition of purified yeast Tif3,or mammalian eIF-4B. In vivo translation of $\beta$ galactosidase reporter mRNAs with varying degree of RNA secondary structure in the 5" leader region in a TIF3 gene-disrupted strain show preferential inhibition of translation of mRNA with more stable secondary structure.

\section{S06-08}

METALLOTHIONEIN SYNTHESIS AND ITS CONTENT IN HUMAN LIVER CELLS

Studer, R. and Hunziker, P. E., Biochemisches Institut der Universität Zürich, $\mathrm{CH}-8057$ Zürich

Metallothioneins (MTs) are small Cys-rich proteins which are inducible by a variety of agents such as bivalent transition-metal ions, tumor promoters, cytokines and hormones. The biological role of MTs is still unclear. Initially postulated to serve in the sequestration of the toxic heavy metals such as cadmium and mercury, they are now believed to play a major role in the cellular metabolism of essential metals such as zinc and copper. To explore this function further we have now established a method to quantify basal MT production in several human cell lines. Thus, cellular isoMT contents were monitored by h.p.l.c. and the rate of synthesis was followed by the incorporation of ${ }^{35} \mathrm{~S}$-cysteine. The results show that maximum MT synthesis and accumulation occurs when the cells enter the exponential growth phase. With increasing celldensity the MT content decreases progressively until confluence is reached. In correlation with preliminary measurements our results suggest a close relationship between the MT content, the cell growth rate and the intracellular abundance of zinc.

\section{S06-09}

\section{PLEIOTROPIC EFFECTS OF HIGHLY PURIFIED SEMLIKI FOREST VIRUS (SFV) NUCLEOCAPSID (C) PROTEIN EXPRESSED BY THE RECOMBINANT BACULOVIRUS ACNPV-C IN SPY INSECT CELLS.}

Favre, D. ${ }^{1}$, Pavlovic, $\mathrm{J}^{2}$ and Michel, M.R. ${ }^{1}$

${ }^{1}$ Institute of Medical Microbiology, University of Berne, CH-3010 Berne

2 Institute for Immunology and Virology, CH-8006 Zürich.

We have successfully purified the recombinant $C$ (recC) protein from spodoptera frugiperda (Sfg) insect cells which were infected with AcNPV-C (A). The recc protein retained the biological activities of native $C$ protein obtained from wild type SFV (B).

our results suggest that the $C$ protein is responsible for the cellular shut-off of host protein synthesis by inhibiting the initiation of translation.

We are currently investigating the molecular mechanisms involved in this shut-off.

(A) Favre, D. et al. Arch.virol, 132:307-319 (1993)

(B) Elgizoli, M. et al. J.virol. 63:2921-2928 (1989)

\section{S06-10}

ANALYSIS OF NUCLEAR IUS GENES CODING FOR THE CHLOROPLAST TRANSLATION ELONGATION FACTOR CEF-G IN SOYBEAN. J.Hernandez Torres, P. Chatellard and $E$. stutz, Laboratoire de Biochimie végétale, Université ōe Neuchâtel, Chantemerle 18, 2000 Neuchâtel

Chloroplast protein synthesis requires the import of elongation factors EF-Tu (tuf-gene) and EF-G (fusgene). It seems that the expression of both genes is light regulated. We recently cloned and sequenced a chloroplast specific nuclear fus gene in soybean (Biochim. Biophys Acta 1174: 191-194, 1993). Further analysis revealed that a second very similar (sequence, anatomy) nuclear fus-2 gene exists which, kowever, shows strong sequence diversion in the 3'trailer region. Several cDNA clones were partially sequenced but sofar only transcripts from the fus1 gene were retrieved. We currently study the promoter regions of either gene to test possible differential expression of fus genes. Comparative mapping and sequencing studies of the two genes in the $3^{\prime}$-region indicate that a major DNA insertion occurred distal to the coding part of both fus genes what may also influence gene expression. 
S06-11

THE RNA-BINDING DOMAINS IN TEE $9 \mathrm{KD}$ AND 14KD SUBUNITS OF THE SIGNAI RECOGNITION PARTICLE HAVE A PUTATTVE O-HELTCAL SMRUTCTURE.

Nazarena Bui and Kathartna Strub

Dpt de Biologie cellulaire, Science III, Université Genève

The signal recognition particle (SRP), a cytoplasmic ribonucleoprotein is important for targeting nascent secretory proteins to the endoplasmic reticulum. SRP9 and SRP14 are constituents of SRP and are, together with the Alu sequences of SRP RNA, required for the elongation arrest activity of the particle. SRP9 and SRP14 form heterodimer in the absence of the RNA and bind the RNA exclusively as a heterodimer. The pritnary secuence of SRP9 and SRP14 revealed that both proteins are highiy basic and lack structural similarities to other characterized RNA-binding proteins. We have therefore been inter

The analysis of $\mathrm{N}$ - and $\mathrm{C}$ - terminal deletion mutants of the proteins have shown that both proteins contribute to the formation of an RNAbinding pocket.

The RNA-binding and dimerization domains in both proteins, are found in regions that have a predicted a-helical structure. In SRP14, this $a-h e l i c a l$ region is located betwen a 72 and 100 at the c-terminus; it contains the RNA binding and djmerization domains adjacent to each other. Our results support the model that the hydrophobic face of the putative $\alpha$-helix is implicated in protein-protein interactions and the positively charged face in RNA-protein interaction. In SRP9, the two RNA-binding domains found N- and C- terminally, also have a predicted amphipatic $\alpha$-helical structure.

S06-12

AGE-RELATED DECLINE OF IRNA IN DROSOPHILA MELANOGASTER

Shikama, N. and Brack, Ch., Biocenter, Dept. of Cell Biologyl, University of Basel, CH-4056 Basel

An age-related decline in protein synthesis is one of the characteristic and common phenomena in most organisms, including $D$. melanogaster.

Being consistent with this observation, a decline in components of protein synthesis machinery during aging has been reported at protein and mRNA levels.

Here we show the age-related decline in rRNA during development and aging, quantitated by Northern blot analysis.

A change of rRNA was compared with that of other protein synthesis machinery components, such as ribosomal proteins and elongation factor $1 \alpha$. A functional activity of ribosome was determined by in vitro poly(Phe) synthesis assay. It indicated the decline in ribosomal function during aging, as well.
S06-13

THE TRANSGENIC FLIES CONTAINING AN ADDITIONAL EF-1 $\alpha$ GENE LIVE LONGER, BUT DO NOT EXPRESS MORE EF-1 $\alpha$.

Ruedi Ackermann, Noriko Shikama, and Christine Brack, Dept. of Cell Biology, Biocenter, University of Basel, CH-4056 Basel

It has been proposed that the decline in protein synthesis observed in aging organisms may result from a decrease in the protein synthesis elongation

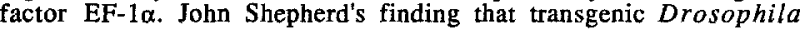
melanogaster carrying an additional copy of the EF- $1 \alpha$ gene have an extended lifespan further indicated that the EF- $1 \alpha$ gene may play an important role in determining longevity (Shepherd et al., 1989). In order to test this hypothesis, we have quantitated EF-1 $\alpha \mathrm{mRNA}, \mathrm{EF}-1 \alpha$ protein, as well as catalytic EF-1 $\alpha$ activity in John's flies. Young and aging EF-1 $\alpha$ transgenic (EF) and control lines (C) were examined. Because the the additional EF-1 $\alpha$ copy is under the control of the hsp70 promoter, flies were aged either at $25^{\circ} \mathrm{C}$ or at $29^{\circ} \mathrm{C}$. The results show that transgenic EF flies do not express more EF-1 $\alpha$ than the control flies, neither at the RNA nor at the protein level.

The question arises, whether the transgene can be induced at all. This was tested by doing RNase protection assays. Transgenic message can be detected only in EF flies heat shocked at $37^{\circ} \mathrm{C}$, but not in EF flies grown at $29^{\circ} \mathrm{C}$, nor in control flies at $37^{\circ} \mathrm{C}$. From this experiment we conclude that the transgene is indeed inducible, but is not expressed in detectable amounts at $29^{\circ} \mathrm{C}$

We conclude that the difference in the lifespan does not result from the overexpression of the EF-1 $\alpha$ transgene.

S06-14

THE MECHANISM OF HEPATIC ENZYME INDUCTION BY PHENOBARBITAI

F.Früh, U.zanger and U.A.Meyer; Biocenter of the University, Department of Pharmacology, CH-4056 Basel

Phenobarbital (PB) induces the expression of liver enzymes involved in the metabolism of drugs and other foreign compounds (xenobiotics). These enzymes include several cytochromes P450, NADPH:P450 reductase, aldeyde dehydrogenase, epoxide hydrolase, UDP-glucoronyltransferases and glutathione-S-transferases. Many other proteins not yet recognized may however be induced by $\mathrm{PB}$. Our first approach therefore is aimed at identifying gene products which respond to PB treatment. As model systems we are using either chicken embryos (induction in ovo) or primary cultures of chicken embryo hepatocytes (induction in culture) (Althaus et al., JBC 254 pp 2148, 1979). To differentially display mRNA of induced vs non-induced cells we are using a RT-PCR technique with sets of random primers. Electrotechnique with sets of random primers. Electrophoretic separation of amplification products allows
to identify mRNA species which are induced (or decreased) following treatment. Data obtained from these experiments and sequence information revealed from extracted PCR products indicate that PB treatment results in the transcriptional avtivation (or repression) of a variety of so far unknown genes. 


\section{Angiogenesis}

S07-01

ANTITHROMBIN - BINDING HEPARAN SULFATES FROM OVARIAN GRANULOSA CELLS

Hosseini G., de Agostini, A., Clinique de Stérilité, HCUG, Genève.

At the time of ovulation, several serine proteases of the plasminogen activator and coagulation cascades are activated in the ovary. These proteolytic activities are tightly controlled in time and space, and the heparin-activated serpins plaminogen-activator-inhibitor-1, protease nexin- 1 and antithrombin (AT) are present in the follicular environment. We have observed that granulosa cells produce a subset of heparan sulfate proteoglycans that bind and activate AT. These AT-binding heparan sulfates (aHSPGs) endow the vascular endothelium with antithrombotic properties. We are now examining the role of aHSPGs in the extravascular compartment formed by the ovarian follicle. Using ${ }_{125}$ I-AT binding assays, we have detected aHSPGs on granulosa cell surfaces and culture media, in amounts comparable to those found for endothelial cells. After $48 \mathrm{~h}$ of stimulation by the gonadotropin FSH (1$100 \mathrm{ng} / \mathrm{ml}$ ) granulosa cells increased the amounts of aHSPGs they released in culture media by 2-6-fold, while keeping their cell surfaceassociated aHSPGs constant. Analysis of ${ }^{35} \mathrm{~S}$-labelled HSPGs revealed that heparan sulfates constitute about $40 \%$ of the surface-associated and $10 \%$ of the soluble granulosa cell glycosaminoglycans. Finally, using 125I-AT autoradiography on rat ovary cryosections, we have localized aHSPGs on the granulosa cell layers of large antral follicles, while aHSPGs could not be detected in smaller follicles. These data suggest that granulosa cell aHSPGs could be critically located to modulate proteolytic activities in the changing environment of the growing follicle.
S07-02

ANIMAL MODELS OF IRON OVERLOAD

wong A, Jin Y. \& Schnebli H.P. Pharmaceuticals Division, CIBA Base1, Switzerland.

The development of chelators which can mobilise excess storage iron (Fe) and permit its excretion is necessary in the treatment of $\mathrm{Fe}$ overloaded diseases. Although oral administration of such a pharmaceutical is highly desirable, no orally active Fe chelator is so far in regular clinical use. Searches for orally active and safe $F e$ chelators require appropriate animal models of Fe overload in order to evaluate the potential of these drugs. An animal model is particularly useful for testing the capacity of new Fe chelating compounds in enhancing the mobilisation of clinically relevant storage iron. Iron loading in animals was achieved by dietary supplementation with carbonyl Fe, Fe fumarate and 3,5,5-trimethy lhexanoyl ferrocene (TMH-ferrocene). Liver Fe concentrations were increased 6-21 times above normal levels. TMH-ferrocene was the most efficient compound to induce stable liver Fe overload. The orally given compounds induced a predominantly hepatocellular iron distribution and was found distributed among reticuloendothelial cells only at a later stage. This pattern of liver $\mathrm{Fe}$ storage is similar to that observed in the early stages of primary haemochromatosis and late stage of transfusional haemochromatosis.

\section{Application of Molecular Biological Principles to Clinical Oncology}

S08-01

FATTY ACIDS AND RETINOIDS CAN ACTIVATE GENE TRANSCRIPTION THROUGH ESTROGEN RESPONSE EIEMENTS

H. Keller, F. Givel, M. Perroud, C. Carlberg* and w. Wahli Institut de Biologie animale, Uni Lausanne, 1015 Lausanne *Clinique de Dermatologie, Hôp. Cantonal Univ. 1211 Genève.

Recently, we and others have cloned and characterized novel transcription factors called perosisome proliferator-activated receptors (PPAR). They belong to the superfamily of nuclear hormone receptors as the steroid, thyroid and retinoid hormone receptors. PPARs are activated, beside xenobiotic peroxisome proliferators, by natural fatty acios and induce, together with the receptor for g-cis retinoic acio (RXR), the transcription of genes involved in the $\beta$ - and w-oxidation of fatty acids. PRAR and RXR bind as heterow-oxidation of fatty acids. PRAR and RXR bind as hetero-
dimers to the PPAR response element (PPRE), consisting of a dimers to the PPAR response element (PPRE), consisting of a
direct repeat of AGGTCA-like sequences with one intervening nucleotide. Now, we show by gel retardation analysis and transient transfection assays that PPAR/RXR heterodimers also bind to and transactivate gene transcription through estrogen half-sites half-sites separated by three nucleotldes. The selectivity and specificity of the transactivation through an ERE was demonstrated by the testing of various related palindromic and inverted palindromic repeats of AGGTCA sequences, which were all inactive. The possible activation of estrogen re sponsive genes by fatty acids via pPARs in vivo is discussed especially with regard to a debated role of fatty acids in breast cancer.

\section{\$08-02}

FAST, COMPLETE AND PERSISTENT ERRADICATION OF PAPILLOMA WARTS AS ADDITIONAL ACTIVITY OF AN ANTI-INFECTIVA AGAINST RNA VIRUSES.

Ludmila E. Wirth-Bronkowska* and Urs V. Wirth* I; *aiRVi-Institute, Höhenweg 7, CH-5426 Lengnau; 7 Biol.Med.Inst., Sonnsyterain, $\mathrm{CH}-6048$ Horw.

- Originally an anti-infectious drug against RNA viruses (aiRVi) was developed. Two different entities such as a biological and a chemical activity unit are necessary for efficiency of aiRVi. This antivirai drug could be used for disinfection, prevention and local therapy of RNA virus infections. Model systems will be devoleped, such as disinfection for hepatitis $A$, prevention against HIV causing pandemics AIDS and local therapy against tumor RNA viruses. - During test phase of a $i R V i$ in compatibility studies, a volunteer asked to get treatment of a permanent papilloma wart, that had not been erradicated with standard medical techniques up to that moment. Four days after the lasi operative excision of this wart on the finger, it was obvious that the virus was establishing a new wart in the centre of the wound again. At this time point, aiRVi was applied twice a day on the wound for two days. During this short time period the developing new wart was completely erradicated and never retumed again now up to 3 month. The whole therapeutic procedure is recorded on video 8 tape, such tapes function as optimal, handy and cheap audio-visueli lab-journais, which can be used later as proof and for docunentation. - in conciusion, beside the high potential of a $R^{R} V i$ against RNA virus infections, it seems that aiRVi is effective for DNA virus infections as well. Thus it would be interesting to examine, if other DNA virus infections (e.g. herpesvinuses) or other localized tumors (e.g. skin tumors) could be treated as well
S08-03

RPMI 8226 HUMAN MYELOMA CELL DEVELOP ATYPICAL MULTIDRUG RESISTANCE WHEN MIMICKING CLINICAL DOXORUBICIN TREATMENT IN VITAO

Wyler B., Shao Y., Schneider E.*, Twentyman P.R. ${ }^{\circ}$ and Lehnert M. Kantonsspital St. Gallen, Onkol. Forschungsabteilung, $9007 \mathrm{St}$. Gallen, * Medicine Branch, National Cancer Institute, Bethesda, MD 20892, USA, - Medicine Clinical Oncology \& Radiotherapeutics Unit, Cambridge, $\mathrm{CB} 22 \mathrm{QH}$, UK

The purpose of this study was to evaluate whether intermittent doxorubicin (DOX) treatment in vitro selects for multidrug resistance in RPMI 8226 myeloma cells and, if so, to determine the underlying mechanism(s). Cells were exposed to constant doses of DOX for 4 days alternating with growth in DOX-free medium for 17 days. After $>9$ months of treatment, the cells developed an atypical MDR phenotype with 4- to 6-fold resistance to topo II poisons. Cross-resistance to vincristine and taxotere was < 2 -fold, sensitivity to cisplatin and melphalan remained normal. Efflux of MDR1 drugs was incresed with no effect of verapamil; subcellular DOX distribution was normal. Expression of topo $11 \alpha$ was found to be reduced by around 50 and 60 $70 \%$ at the RNA and protein level, respectively. Minimum MDA1 RNA overexpression was detected when using RT-PCR; P-glycoprotein was not detectable. MRP RNA expression was increased by $60-90 \%$ relative to the wild type cells without detectable p190. This data suggests that atypical MDR might play a role in acquired DOX resistance in human myeloma.

\section{S08-04}

PRODUCTION IN BACTERIA OF RECOMBINANT HUMAN PROCATHEPSIN $B$ AS A FUSION PROTEIN TO GLUTATHIONE S-TRANSFERASE.

Keppler, D.; Bachmann, D.; Chan, S.J.; Berdoz, J.; Astori, M.; and Sordat, B.; Experimental Pathology Unit, Institute for Experimental Cancer Research (ISREC), 1066 Epalinges, Switzerland; Fax: 021/652-6933.

The proteolytic enzyme, cathepsin $B$, has been implicated in a variety of pathophysiological processes, such as inflammatory and autoimmune diseases, neurodegenerative diseases, muscular dystrophy, and the malignant progression of tumors. With the aim of producing specific tools (such as monoclonals) for the study of this enzyme in tumor invasion, we have produced recombinant human procathepsin $B$ in bacteria as a fusion protein to glutathione $\mathrm{S}$-transferase. The nearly ful1 length human procathepsin $B$ CDNA, from phCB 79-1, was subcloned into the expression vector pGEX-2T. Clones, one positive and one negative, were selected by colony hybridization, plasmid mini-prep analysis, SDSPAGE after IPTG-induction, and western blotting using polyclonals to human cathepsin $B$. 
S08-05

SYSTEMS FOR EXPRESSION OF SECRETORY COMPONENT

Lorenz Rindisbacher, Riccardo Wittek, and Blaise Corthésy

Institut de Biologie animale, Université de Lausanne

Mucosal surfaces cover a vast area in the human body. They satisfy its need to communicate with its environment, e.g. in terms of nutrient uptake and sexual reproduction. However, at the same time they offer a vulnerable gate for invasion by pathogenic microorganisms. For immunoprotection of these regions, large amounts of $\lg \mathrm{A}$ antibodies are produced in the bloodstream and transported across the epithelial barrier to the lumen by means of polymeric ig receptor. There the receptor is cleaved, and its extracellular portion remains associated as secretory component (SC) to the secretory IgA complex.

In the context of a project aiming to mass production of secretory $\lg A$ as a passive vaccine, we evaluated a series of eukaryotic expression systems for production of SC. The CDNA encoding polymeric $\mathrm{lg}$ receptor was engineered in a way that only its extracellular domains corresponding to $\mathrm{SC}$ were expressed in yeast, in insect cells infected with recombinant baculovirus, and in mammalian cells infected with recombinant vaccinia virus. Furthermore, a C-terminal poly-histidine tag was introduced for simple purification of the products. We will show optimization of expression levels for each of the systems as well as a quantitative and qualitative comparison of the products.

\section{S08-06}

USE OF VACCINIA VIRUS RECOMBINANTS TO PRODUCE SECRETORY COMPONENT

S. Cottet, B. Corthésy, L. Rindisbacher and R. Wittek Institut de Biologie animale de l'Université de Lausanne

Recombinant vaccinia viruses (rVV) that express gene products from other organisms has become a popular and widely used laboratory expression system. Our current interest is directed toward the production of secretory component (SC), a subunit of secretory $\operatorname{IgA}$ antibody complex involved in mucosal immunity. Toward this goal, we constructed rVV governing SC transcription under the control of two viral-based and the T7 bacteriophage promoters. Expression of the protein was assessed in several mammalian cell lines, such as human TK-, human HeLa (grown in suspension or as a monolayer) and monkey $\mathrm{CV}-1$. Optimization of infection parameters and culture conditions were also performed.

\section{S08-07}

\section{METHYLATION OF CPG'S IN THE CHICKEN $\alpha 2$ (VI) COLLAGEN GENE}

Kopp, M., Willimann, T. and Trueb, B.; Biochemie 1, ETH Zürich

Enzymatic methylation of cytosines in mammalian DNA is believed to play a fundamental role in basic gene regulation. Methylation in the vertebrate genome is restricted to $\mathrm{CpG}$ dinucleotides at the $\mathrm{C}-5$ position of cytosine. In vitro studies have shown that methylation can drastically influence the DNA structure. CpG islands remain unmethylated in normal cells, whereas several immortalised, transformed cell lines contain , whilly methylated CpG islands. transformed cell lines contain partially methylated CpG islands. Transcription of genes can be inhibited by methylation of CpG's. This
modification contributes $\theta . g$. to the stable repression of genes on the modification contributes $\theta . g$. to the stabl
inactivated $X$ chromosome of females.

The collagen VI genes show typical CpG islands in their promoter region and they were shown to be down regulated at the transcriptional level in src or myc transformed cells. Given these facts we decided to investigate the effects of methylation in the collagen VI we decide

gene.

We could demonstrate that the promoter activity of $\alpha 2(\mathrm{VI})$ chicken collagen gene was strongly diminished by methylation in vitro. Furthermore DNA methylation completely prevented binding of a novel transcription factor to the promoter, whereas binding of SP1 was not affected.

To show evidence of methylation in vivo, we are currently localising the exact positions of methylated CpG's in normal and transformed cells by genomic sequencing.
S08-08

BIOCHEMICAL CHARACTERISATION OF RECOMBINANT SECRETORY COMPONENT

Blaise Corthésy, Riccardo Wittek and Lorenz Rindisbacher

Institut de Biologie animale de l'Université de Lausanne

Protection of mucosal surfaces is mediated by secretory IgA antibodies ( $\lg A$ ) constituted of dimeric $\lg A$ and secretory component (SC). Toward the aim of reconstituting $\operatorname{sigA}$ complexes in vitro and using them for passive ora immunization, SC has been produced in yeast, insect and mammalian expression systems. The gene encoding SC has been engineered so that a) the C-terminus of the protein carries a $6 \mathrm{xH}$ is tag and $b$ ) the newly synthesized polypeptide is released in the culture medium. Culture conditions have been established to permit recovery of SC in serum-free medium. Purification procedures based on nickel chelate and classical chromatographies have been optimized to yield SC under native conditions. Finally, in vitro reassociation with purified dimeric $\lg$ As obtained from hybridomas has been used to assess biological activity of recombinant SC.

\section{S08-09}

ISOLATION OF PUTATIVE NON-SMALI CEIL LUNG CANCER SUPPRESSOR GENES BY A MAGNET-ASSISTED SUBTRACTION HYBRIDISATION TECHNIQUE,

Peter Schraml, Rob Shipman, Marco Colombi and Christian U. Ludwig, Molecular Oncology, Zentrum für Lehre und Forschung, Basel, Switzerland.

Using a Magnet-Assisted Subtraction-hybridisation Technique (MAST), 17 cDNA clones were isolated that are expressed in normal lung tissue but not expressed in the correspending NSCLC tissue. 11 of the 17 cDNA clones are highly homologous to CDNA sequences for the following proteins: Pulmonary surfactant proteins $S P-A$ and SP-B; Receptor for Advanced Glycosylated Endproducts of proteins (RAGE); Calmodulin-like protein: Natural killer gene 5 (NKG5); Matrix Gla protein (MGP); Glutamine synthetase; Ubiquitin; Vascular smooth muscle alpha-actin; Cytoskeletal beta-actin; Vimentin. The remaining 6 cDNA clones may represent parts of still unknown genes. The MAST cDNA clones were derived from a patient who developed squamous adenocarcinoma. Northern blot analysis of normal/tumour RNA from three other NSCLC patients showed that the lack of gene expression was independent of NSCLC type and stage.

\$08-10

ALTERATIONS OF RUTATIVE TUMOUR SUPPRESSOR GENES IN HUMAN NON-SMALL CELI LUNG CARCINOMA (NSCLC). R. Shipman and C.U. Ludwig, Molecular oncology, Lab 405 (ZLE), Basel, CH-4031

Chromosomal subregion 11 pl3 displayed non-random allelic loss in 61 NSCLCS. 11 p13 contains the genetic Ioci for catalase (CAT), the Wilms' tumour gene (WT1) and the folliclemstimulating hormone $B$-chain (ESE $\beta$ ). $76 \%$ of the NSCLCS were deleted at CAT suggesting that loss of a gene(s) near CAT may be involved in the progression of NSCLC. YAC clones containing the CAT locus are being prepared for use in a direct selection procedure for cDNAs encoded at this region. Although $38 \%$ of the NSCLCs were deleted at WT1, no mutations were detected in the remaining WT allele. WT' expression was subsequently demonstrated in fetal lung, fetal hematopoietic tissue, normal adult $\mathrm{lung}$ and NSCLC. Allelic deletion at 17pi3, immuno-histochemical and sequence analysis of the p53 gene were also examined in our NSCLCS. These analyses support the contention that aberrant expression of the p53 gene is a pivotal event in the progression of NSCLC. 


\section{LOW LEVELS OF RADIATION EXPOSURE FAIL TO INDUCE CELIULAR RECOVERY MECHANISMS: STUDIES OF MUTATION, SURVIVAI AND CANCER INDUCTION.}

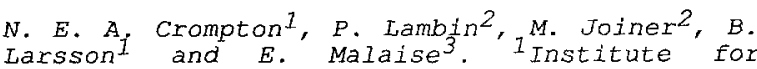
$\begin{array}{ll}\text { Larsson and E. Malaise } & \text { Institute for } \\ \text { Medical Radiobiology, } 5232 \text { Villigen-PSI, }\end{array}$ Switzerland; ${ }^{2}$ CRC Gray Laboratory, Mount Vernon Hospital, Northwood, Middlesex, UK; ${ }^{2}$ Institut Gustav-Roussy, 94805 Villejuif, Cedex, France.

Recently, a number of advances have been made which enable the experimentalist to study the effects of low levels of ionizing radiation. The results suggest a threshold to radiation damage exists and that above a critical level, recovery mechanisms in the cell are induced and the biological response falls. Thus low levels of radiation cause greater than expected levels of biological response. This has been demonstrated in studies of mutations, cell survival and cancer induction. Because the low dose-rates and the low doses per fraction result in the total doses being small, the magnitude of the biological effects induced is insufficient to warrant alarm.

S08-12

Cloning of T-lymphocytes from patients with deficient synthesis of the glycosylphosphatidylinositol (GPI)- anchor

$K$. Mrkoci, A. Dinter, M. Thurnher and E.G. Berger, Institute of Physiology, University of Zurich

Deficient synthesis of the GPI anchor is the molecular basis of idiopathic paroxysmal nocturnal hemoglobinuria ("PNH") and a similar phenotype can accompany aplastic anemia ("PNH-like"). The population expressing CD48, a GPI-anchored membrane glycoprotein, was quantified by flow cytometry in samples from patient P.G. (PNH) and patient M.M. (PNH-like) and amounted to 90\% (P.G.) and 78\% (M.M.), respectively. T lymphocytes from both patients were isolated, expanded and cloned. From both patients, clones expressing the CD48 marker (CD48+) and CD48 deficient clones (CD48-) were obtained.

Takeda et al. (Cell 73, 1993, 703-11) showed that in some (CD59-) Bcell clones, a deletion of the PIG-A gene product is responsible for the expression of the PNH-phenotype. PCR analysis of T cell mRNA from extracts of both CD48+ and CD48- clones of patient M.M. (PNH-like) using PIG-A specific primers revealed a band pattern from which can be concluded that no deletion is present in the PIG-A gene product. This result suggests that CD48- T lymphocytes of the PNH-like patient express a normal PIG-A gene product; a disorder in regulation of PIGA expression or an unrelated biosynthetic defect may explain the CD48phenotype in these $T$ cells. Further work is aimed at defining the differences in biosynthetic defects of PNH and PNH-like CD48- T cell clones. Supported by grant 31-30757-91 of the SNSF to EGB.
Identification of genes associated with the revertion of the malignant phenotype of a rhabdomyosarcoma cell line.

Michele Genini, Sabina Solinas Toldo*, Ruedi Fries* and Beat W. Schäfer, Dept. of pediatrics, University of Zürich, Steinwiesstr. 75 , 8032 Zürich, and *Institut für Nutztierwissenschaften, ETH Zürich, 8092 Zürich.

The human rhabdomyosarcoma cell line RD is tumorigenic and differentiates very poorly despite the expression of the myogenic factors myf3 and myf4. Therefore it can be regarded as natural mutant. To identify genes which are involved in suppression of tumorigenicity or enhance differentiation we applied two different approaches.

Since the development of human embryonal rhabdomyosarcoma has been associated with abnormalities of chromosome 11 band p15, in the first approach we transferred a normal human chromosome 11 into RD cells via microcell fusion. The microcell hybrids did not show a higher degree of differentiation. Suppression of tumorigenicity was observed in one clone but is probably independent of chromosome 11 , as it was shown by chromosome 11 specific painting.

In the second approach we applied a subtractive hybridization procedure between primary myoblasts and RD cells to find genes specific for the normal phenotype. These genes will be tested in vivo for induction of differentiation and/or tumorsuppression.
S08-15

MODULATION OF THE POLARIZED EPITHELIAL PHENOTYPE DURING TUMORIGENESIS

E. Reichmann, J. Peli, M. Oft, E. Deiner, H. Beug Institute of Molecular Pathology, Dr. Bohr-Gasse 7 , vienna We have recently employed an experinental approad to turn the function of "nuclear messengers" such as c-Fos (and c-Jun) on and off at will in polarized mammary epithelial cells. To this end we used constructs encoding the c-fos (and c-jun) genes fused to the hormone-binding domain of the buman estrogen receptor, designated c-FosER (and C-JunER). We could show that short-term activation (30 mins.) of C-FosER by estradiole (E2) led to the disruption of epithelial cell polarity within 24 hours, as characterized by the expression of apical and basolateral marker proteins. During the next 3-5 days, however, the Dever, the cells fully regained their polarized phenotype. Conversely, long-term activation (24-48 hours) caused the ul ultinately to an epithellal fibroblastoid cell transition. These cells underwent dramatic changes in gene expression including the complete loss or reduction of epithelial markers such as E-cadherin/uvomorulin, 20-1 and cytokeratins, and the de novo expression of mesenchymal proteins like vimentin, type I collagen and fibronectin. expression of the $\mathrm{v}$-Ha-ras oncogene lacting upstream of $\mathrm{c}$ Fos) did not markedly influence epithelial cell organization when the cells were grown on plastic. However, when cultured within type $I$ collagen gels in the presere wh cultured within type presence of serum, or when injected into the mammary epithelial-fibroblastoid cell transition was observed.

\section{S08-13}

Growth properties determine the organ colonization ability of a metastatic murine melanoma cell line.

Dario Rusciano, Patrizia Lorenzoni and Max M. Burger. Friedrich Miescher Institut, P.O. Box 2543, CH-4002 BASEL.

The ability of metastatic tumor cells to specifically colonize distant organ sites strongly depends on the interaction of the metastatic cells with the local organ environment. We have selected a B16 murine melanoma cell line (B16-LS9) which has an increased ability to colonize the liver, and compared its behavior with either its parental (B16-F1) or lung-specific (B16-F10) cell line. We have found that adhesion in vitro and organ lodgement in vivo were not different for LS9 and F10. In contrast, B16-LS9 grew at slower rates than the other lines both in vitro and in vivo after subcutaneous or intra-foot-pad injection. Analysis of tyrosine-phosphorylated proteins indicated a higher phosphorylation activity in B16-LS9 cells, which might suggest an altered regulation of some kinase(s) in this cell line. Intercellular contact with hepatocytes in vitro, or the liver in vivo could restore an efficient growth of B16-LS9, enabling thus these cells to colonize this organ much better than others. Hepatocytes or liver plasma membrane (p.m.) extracts conserved the growth stimulatory activity towards B16-LS9 cells. Chromatographic fractionations of these extracts showed that a major growth stimulatory protein in this liver p.m. fraction turns out to be transferrin, which in the liver appears to exist in at least two different mature forms. 


\section{From Whole Body to Cellular Energetics}

\section{S09-01}

THE ENERGETICS OF MITOCHONDRIAL PROTEIN IMPORT B.S. Glick, C. Wachter and G. Schatz. Biocenter, Klingelbergstrasse 70, CH-4056

Basel, Switzerland.

Protein import into mitochondria requires ATP in two locations: outside the organelle, and in the matrix space. Depletion of matrix ATP arrests translocation of precursors across the inner membrane, but does not prevent precursors from crossing the outer membrane. As a result, proteins that reach the inner membrane or intermembrane space without passing through the matrix are often sorted normally in ATP-depleted mitochondria. External ATP is used to maintain precursors in a translocationcompetent state. However, some precursors can fold and still remain translocation-competent. and import of these precursors is independent of external ATP. With the folded cytochrome $b_{2}$ precursor, it is matrix ATP rather than external ATP that drives translocation across the outer membrane. Thus matrix ATP generates a pulling force that can drive both the translocation and the unfolding of precursor proteins. Mitochondrial hsp70 probably functions as the ATP-dependent import motor.

\section{S09-02}

Identification and functional analysis of chaperonin 10 , the groES homolog of yeast mitochondria.

S. Rospert, T. Junne, B. Glick and G. Schatz

Biozentrum, University of Basel, Klingelbergstrasse 70, $\mathrm{CH}-4056$ Basel Switzerland. Phone ++41-61-2672171, FAX ++41-61-2672148.

The mitochondrial chaperonin system consists of chaperonin 60 (cpn60; also termed hsp60), which is homologous to $E$. coli groEL, and chaperonin 10 (cpn10), which is homologous to E. coli groES. We have used a functional assay to identify cpn10 of yeast mitochondria. When dimeric Rubisco is denatured and allowed to bind to yeast cpn 60 , subsequent refolding of the enzyme is strictly dependent upon yeast cpn10. In the presence of $\mathrm{MgATP}$, yeast cpn 60 and yeast cpn10 form a stable complex that can be isolated by gel filtration. The ATPase activity of hsp60 is not inhibited by complex formation with cpn10. A different result is obtained with the $E$. coli system, where groES is able to completely inhibit the ATPase activity of groEL (1).

To test the in vivo role of cpn10, we have cloned, sequenced and disrupted the corresponding nuclear gene CPN10. This gene encodes a protein of $11,372 \mathrm{Da}$ that is imported into the mitochondrial matrix without detectable cleavage. Haploid cells lacking a functional copy of CPN10 fail to grow at tempetatures between $23^{\circ} \mathrm{C}$ and $37^{\circ} \mathrm{C}$ (2).

(1) Rospert, S. et al. (1993) Proc. Natl. Acad. Sci. 90, in press.

(2) Rospert, S. et aI. (1993) FEBS Lett., in press.

\section{S09-03}

Characterization of yeast mitochondrial heat shock protein 70, L. Bolliger, B. S. Glick and G. Schatz, Biocenter, University of Basel, 4056 Basel.

Mitochondrial hsp70 (mhsp70) is thought to use the energy of ATP hydrolysis to pull precursor proteins across the mitochondrial membranes. To facilitate the purification of yeas mhsp70, we used the cloned gene (a gift of Dr. N. Morishima) to modify mhsp70 with a six-histidine tag at its C-terminus. This construct supports growth of yeast cells lacking wild-type mhsp70. We developed a purification procedure that allows is to recover this tagged protein with a purity of about $95 \%$ Experiments are in progress to characterize the nucleotidedependent interaction of purified mhsp70 with unfolded proteins. In addition, we are looking for partner proteins that may modulate the action of mhsp70. We have copurified a protein of about $20 \mathrm{kD}$ together with mhsp 70 . The $20 \mathrm{kD}$ protein could be released from mhsp70 by ATP or ADP. When tryptic fragments of this protein were subjected to microsequencing, one fragment showed strong homology to the GrpE protein of E.coli.
S09-04

\section{METABOLIC RATE AND IMMUNE STATUS OF MICE CHRONICALLY INFECTED} WITH TOXOPLASMA GONDII

Arsenijevic D, Dulloo AG \& Girardier L, Dpt of Physiology, CMU, Geneva, CH. The relationship between body weight, daily energy expenditure (assessed by indirect calorimetry), and immune status (determined by lymphocyle proliferation tests) was investigated in Swiss Webster mice maintaining a stable body weight several weeks after infection with Toxoplasma gondii (Me49). Following an initial period of weight loss (infection-induced cachexia), the surviving mice could be differentiated into two main groups: (i) those showing a partial regain in body weight (the infected gainers) and eventually stabilizing at a mean body weight $25 \%$ below a non-infected control group, and (ii) those showing no weight regain (the infected non-gainers) and eventually stabilizing at $45 \%$ below control levels. Immune function was found to be markedly suppressed in the infected non-gainers, whereas it was normal (and similar to control levels) in the group of infected gainers. Daily energy expenditure (and food intake), after normalisation for differences in body weight, was found to be the same in both infected gainers and non-gainers, but lower by $15 \%$ when compared to controls $(P<0.01)$; thereby indicating that the infected groups were able to increase their overall efficiency of food utilization in response to the low food intake. In conclusion, this study conducted during a weight stable phase of chronic infection shows a clear-cut association between the inability to regain body weight and impaired immune function. In contrast, no relationship was found between metabolic rate and body weight nor between metabolic rate and immune function, but the chronically infected mice exhibited the same phenomenon of enhanced metabolic efficiency characteristic of chronic underfeeding perse.

\section{S09-05}

METHOD FOR IN SITU MEASUREMENT OF THE MITOCHONDRIAL MEMBRANE POTENTIAL IN SINGLE HEPATOCYTES FROM THE RAT

J.J. Ubl \& J.W. Stucki; Pharmakologisches Institut, Universität Bern, Friedbuihlstr. 49, 3010 Bern

The transmembrane electrical potential of mitochondria $\left(\Delta \Psi_{\mathrm{mit}}\right)$ can be assessed in single hepatocytes by using epifluorescence microscopy. For this end the rhodamine 123 fluorescence of a mitochondria-rich $(\mathrm{Fr})$ and a mitochondria-poor region $(\mathrm{Fp})$ are measured. The ratio of these signals depends on the $\Delta \Psi$ mit according to a modified Nernst equation (Reber B.F.X., Somogyi R. \& Stucki J.W. (1990), BBA, 1018, 190-193). To calibrate this method the cell membrane was permeabilized for monovalen cations with nystatin and gramicidin. Then the cytosolic $\mathrm{K}^{+}$was replaced by $\mathrm{Na}^{+}$from a $\mathrm{K}^{+}$-free bath solution. Subsequent addition of valinomycin made the mitochondrial membrane permeable for $\mathrm{K}^{+}$ions. By the addition of different $\mathrm{K}^{+}$concentrations to the bath, the $\Delta \Psi_{\text {mit }}$ could be clamped to defined values. The relation between the ratio $\mathrm{Fr} / \mathrm{Fp}$ and the $\Delta \Psi_{\text {mit }}$ could be well fitted to our modified Nernst equation. However, the calibration curve flattens out at potentials higher than about $110 \mathrm{mV}$. Therefore the

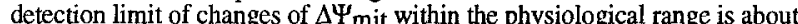
10-20mV. Maximal stimulation of the $\mathrm{Na}^{+} / \mathrm{K}^{+}$ATPase by addition of nystatin to the $\mathrm{Na}^{+}$containing bath medium caused a drop of $\Delta \Psi_{\text {mit }}$ of about $20 \mathrm{mV}$. Subsequent addition of ouabain resulted in an almost complete reversal of this drop. This shows that changes in ATP consumption can be assessed via changes of $\Delta \Psi_{\text {mit }}$

\section{S09-06}

LACK OF A TIGHT CORRELATION BETWEEN INSULIN RESISTANCE AND HYPERTENSION.

Cusin I., Vettor R., Ganten D., RohnerJeanrenaud F., Ferrannini E., Jeanrenaud B. Laboratoires de Recherches Métaboliques, Geneva, Switzerland.

An interrelationship between hyperinsulinemia and insulin resistance has been proposed. Transgenic hypertensive TGR(mREN2)27, insulinresistant obese fa/fa, and normal spragueDawley (SD) rats were studied. In TGR, blood pressure (BP) was higher than in SD rats (203 \pm 8 vs $112 \pm 6 \mathrm{mmHg}, p<0.001)$. In obese rats $B P$ was the same as in SD. Iiver glucose output, peripheral glucose uptake were normal in TGR and SD rats. Obese fa/fa rats had hepatic and peripheral insulin resistance. There is no tight correlation betwen insulin resistance and hypertension. 
S09-07

CHRONIC I.C.V. NPY ADMINISTRATION IN NORMAL RATS : FFFECTS ON METABOLISM.

Rohner-Jeanrenaud F., Zarjevski N., cusin I., Vettor R., Jeanrenaud B. Laboratoires de Recherches Métaboliques, Geneva, Switzerland

Genetically obese rodents have increased hypothalamic Neuropeptide Y (NPY) content. The impact of NPY given i,c.v. to normal rats was thus studied. NPY produced hyperphagia, overweight, hyperinsulinemia, hypercorticosteronemia. It increased liver and white adipose tissue (WAT) lipogenesis. It also resulted in an increase in WAT insulinstimulated glucose uptake as well as GLUT 4 mRNA and protein. In contrast, it resulted in a decrease glucose uptake by muscles without altering GLUT 4 expression but, possibly via a glucose-fatty acid cycle. NPY is thus an important clue in obesity syndromes.

\section{S09-08}

Hormonal regulation of $\mathrm{C}-\mathrm{AbI}$ tyrosine kinase by fusion to a steroid binding domain

T. Mattioni, O. Bchini Hooft van Huijsduijnen, P.K. Jackson and D. Picard Département de Biologie Cellulaire, Université de Genève, $\mathrm{CH}-1211$ Genève 4

The activities of a wide variety of proteins can be subjected to hormonal control by fusion to the hormone binding domain of steroid receptors. We show that this strategy can also be applied to a tyrosine kinase. In particular, transforming derivatives of $\mathrm{c}$-Abl become hormone-inducible oncogenes by fusion to a steroid binding domain. It is noteworthy that the hormone-inducible transformation of NIH3T3 cells is completely reversible upon removal of the specific ligand. Reversion experiments reveal another facet of Abl: its ability to inhibit cell proliferation. 48 hours after hormone depletion, cells are not only morphologically reverted, but the cell cycle appears to be blocked in G1. A cytostatic effect of Abl overexpression has been reported by several groups but, until today, it could only be examined by comparing different Abl derivatives or the same derivatives in different cellular contexts. In contrast, our hormone-regulable system has the advantage that the two distinct functions of Abl (transformation versus growth inhibition) can be studied using the same derivative expresed in the same cell line. We are using the hormone inducible system to investigate the mechanisms regulating the transforming and the cytostatic effects of $\mathrm{Abl}$ in a variety of cell lines.

S09-09

\section{PHOTOACTIVITY OF THE GREEN ALGAE CHLAMYDOMONAS REINHARDTII INCORPORATED IN WATER-IN-OII MICROEMULSION: EFFECTS OF WATER LIMITATIONS}

Kernen, P. ${ }^{1}$, Darszon, A. ${ }^{2}$, and strasser, R.J. ${ }^{1}$,

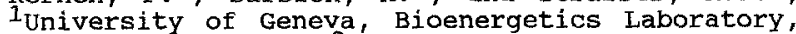
CH-1254 JUSSY and 2 Institute of Biotechnology, UNAM, Cuernavaca, Morelos 6227, Mexico

Light-induced fast fluorescence changes were observed for cells of the green algae Chlamydomonas reinhardtii placed in a reverse micellar solution of hexadecane-Tween 85-span 80 . Under water limitations the fluorescence induction kinetics were different from the typicaliy observed O-J-I-P rise of photosynthetic active oxygenic systems. changing the water concentration from 2 to $6 \%(\mathrm{v} / \mathrm{v})$ in the low water system changed the kinetics of the fluorescence induction curves. Lowering water concentrations also distinctly affected the kinetics of cell mutants. It appears that energy flux to the reaction center is reduced in reverse micelles and that the fluorescence levels, corresponding to reduced quinone forms (e.g. [ $Q_{A}$, $\left.\left.Q_{B}\right],\left[Q_{A}^{-} Q_{B}^{-}\right],\left[Q_{A}{ }^{-} Q_{B}{ }^{2-}\right]\right)$ are markedly affected by lowering the water concentration.
S09-10

EFFECTS OF EXERCISE IN THE FASTED OR FED STATE ON GLYCOGEN KINETICS IN MAN.

Schneiter Ph., Di Vetta V., Jéquier E. and Tappy L. Institut de physiologie de l'Université, Bugnon 7, 1005 Lausanne.

The metabolic effects of an exercise performed in the fasting or fed state were studied in 8 subjects over a 8 hour period in a respiratory chamber. A mixed meal containing ${ }^{13} \mathrm{C}$ labeled carbohydrates was ingested at 9:30 am and an exercise session (walking at $5 \mathrm{~km} / \mathrm{h} 45 \mathrm{~min}$, slope $10 \%$ ) was performed at 8:15 am (fasting) or 11 am (postprandial). Net carbohydrate (net $\mathrm{CHO}$ ox) and lipid oxidation (indirect calorimetry) and oxidation of exogenous carbohydrates (exo $\mathrm{CHO}$ ox, breath ${ }^{13} \mathrm{CO}_{2}$ ) were measured. Glycogen breakdown was calculated as net $\mathrm{CHO}$ ox $\mathrm{CHO}$ exo ox, and glycogen synthesis as $\mathrm{CHO}$ in - CHO exo ox. Energy expenditure over 8 hours was similar but net $\mathrm{CHO}$ ox was $16 \%$ lower, lipid ox $61 \%$ higher and exo $\mathrm{CHO}$ ox $39 \%$ lower when exercise was performed in fasting vs fed state; moreover, glycogen synthesis was increased by $40 \%(p<0.0001)$, while glycogen breakdown was increased by $12 \%(p=0.06)$.

In conclusion, a $45 \mathrm{~min}$ exercise in the fasting vs fed state a) increases utilization of endogenous lipids, b) enhances muscle glycogen turnover over a 8 hour period.

So9-11

\section{THERMOSTABLE MUTANTS OF THE MESOPHILIC TRIOSEPHOSPHATE ISOMERASE OF B. MEGATERIUM. THE ROLE OF THE $\alpha$-HELICES IN THE TIM BARREL}

Jiewu YANG and Herbert Zuber

Institute for Molecularbiology and Biophysic, ETH, 8093 Zürich

Enzymes from thermophilic organisms show higher thermostability and temperature optima than those of mesophilic organisms. It has been shown that these properties are based on a specific structure of the protein. The primary structure of thermophilic (B. stearothermophilus) and mesophilic(B. megaterium) triosephosphate isomerase(TIM) have been determined by cloning and sequencing of the TIM genes. Comparison of the primary structures of the thermophilic and mesophilic enzyme showed specific amino acid substitutions, particularly in the $\alpha$-helices of the TIM barrel, which could play a critical role with respect to thermostability. We have constructed mutants by exchanging $\alpha$-helices between TIM of B. stearothermophilus and B. megaterium. Helix $\mathrm{H} 1, \mathrm{H} 2$ and $\mathrm{H} 7$ of TIM from $\mathrm{B}$. megaterium, corresponding to amino acid residues 13-36, 44-55. and 220-227 respectively, have been exchanged. The properties (thermostability and temperature optimum) of the hybrid-mutants were compared with the wild type enzymes.

\section{S09-12}

IIPID DROPLETS AND MITOCHONDRIA IN SKELETAL MUSCLE CELLS: QUANTITATIVE SEATIAL RELATIONSHIP

R. Vock, H. Claassen, H. Hoppeler, E.R. Weibel

Institute of Anatomy, University of Berne, $\mathrm{CH}-3000$ Berne 9

In a comparative investigation of structural and functional parameters related to the oxidative substrate metabolism in skeletal muscle cells of dogs and goats, a close relationship was found between intracellular lipid stores and

mitochondria. As revealed in serial sections with subsequent EM analysis, each itpid droplet was in direct contact to one or several

mitochondria. Quantitatively, $23 \%$ of the lipid droplet surface in goats and $40 \%$ in dogs were in direct contact to outer mitochondrial membranes or - in other terms - 1\% of outer mitochondrial membranes in goats and $2 \%$ in dogs were in direct contact to lipid deposits.

These findings were in good agreement with the physiological data showing a 2 times higher oxidation rate of fatty acids drawn from intracellular stores for dogs than for goats. 
S09-13 DETERMINATION OF mRNAS AND MTDNA IN SKELETAL
MUSCLES OF UNTRAINED AND ENDURANCE TRAINED MUSCLES

A. Puntschart; K. Jostarndt; H. Hoppeler; R. Billeter Institute of Anatomy, University of Bern, $\mathrm{CH}-3012 \mathrm{Bem}$.

Human muscle adapts to altered load with changes in the expression of enzymes involved in energy metabolism. Endurance exercise in humans is known to increase the oxidative capacity of skeletal muscles (Hoppeler, Int. J. Sports Med. (1986), Z, 187). At the ultrastructural level, a higher mitochondrial content contributes to the higher oxidative capacity of skeletal muscles. Little is known about the molecular mechanisms that lead to such adaptations in humans. The expression of mitochondrial components involves complex regulation of both mitochondrial and nuclear encoded genes. We have developed a PCR approach to quantify DNA and RNA from human skeletal muscle cryostat sections and addressed the question, whether the structural adaptations in endurance trained athletes are accompanied by concomitant changes in respective RNA steady state levels. Preliminary data indicate a higher concentration of COXIV and COXI (1.8 and 1.6 times, respectively) in trained subjects. No differences were observed for mtDNA, despite a two fold higher mitochondrial content.

\section{S09-14}

MYOSIN ALKALI LIGHT CHAIN mRNAS IN NORMAL AND DYSTROPHIC HUMAN SKELETAL MUSCLES.

K. Jostarndt ${ }^{1}$; A. Puntschart ${ }^{1}$; H. Hoppeler ${ }^{1}$; J. Burgunder ${ }^{2}$; R. Billeter. ${ }^{1}$

Institute of Anatomy ${ }^{1}$ and Insel Spital ${ }^{2}$, University of Beme, 3012 Berne.

Human skeletal muscle contains three main fiber types which are based on the myosin heavy chain composition of the individual fiber. The isoforms of other contractile proteins within a given fiber type can vary leading to a continuum of different phenotypes.

We are studying the fiber type specific expression of the mRNAs of myosin alkali light chains (MLC) using in situ hybridization, especially the rarer slow form MLC 1sa. Differences were found between shoulder muscles and leg muscles: $m$. deltoideus yielded stronger signals for MLC 1sa and MLC 1sb in the least glycolytic type I fibers (IA), weak but detectable signals for MLC 1sb and MLC If/f $\mathrm{mRNA}$ in more glycolytic type I fibers (IB) and strong signals for MLC $1 \mathrm{f} / 3 \mathrm{f}$ in type II fibers. In $m$. vast. lat. MLC 1 sb was strongly expressed in type IA as well as IB fibers, MLC 1sa mRNA was only found in some IA fibers.

MLc 1sa has been shown to be expressed at a particular time point during muscle development and regeneration. In a biopsy of a Becker Dystrophy patient, we found very strong signals in some small fibers displaying the morphology of regenerating fibers. Thus this probe could be useful to detect regenerative processes in pathological muscle samples.

\section{S09-15}

ENERGY METABOLISM AND PROTEIN TURNOVER IN OBESE PREPUBERTAL CHILDREN. Rueda-Maza $\mathrm{Cl} \mathrm{M}^{*}$, Maffeis $\mathrm{C}^{* *}$, Zaffanello $M^{* *}$, Schutz $Y^{*}$. "Institut of Physiology. Univ. of Lausanne. Switzerland. *Department of Pediatrics. Univ. of Verona. Italy.

The aim was to examine whole body protein turnover and resting energy expenditure (REE) in obese (OB) prepubertal children $(n=10,44 \pm 4 \mathrm{Kg}$ ), compared to 8 matched controls $(C, 29 \pm 2 \mathrm{~kg}$ ) of the same chronological age $(8.5 \pm 0.2 \mathrm{yr})$. REE was assessed by indirect calorimetry. Whole body protein synthesis (PS) was calculated from the rate of $15 \mathrm{~N}$ urinary ammonia enrichment using a single dose of ${ }^{15} \mathrm{~N}$ glycine. In absolute value, $\mathrm{PS}$ and protein breakdown $(\mathrm{PB})$ were found to be significantly greater in $\mathrm{OB}(208 \pm 24$ vs $137 \pm 14 \mathrm{~g} / \mathrm{d} ; \mathrm{p}<0.05)$ and $(149 \pm 20$ vs $90 \pm 13 / \mathrm{d} ; \mathrm{p}<0.05)$ than in $\mathrm{C}$, respectively. However, when expressed per $\mathrm{kg}$ fat-free mass (FFM), only the $P B$ was significantly greater in $O B$ than in $C(5.0 \pm 0.5 v s 3.7 \pm 0.4 \mathrm{~g} / \mathrm{kgFFM} / \mathrm{d} ; \mathrm{p}<0.05)$. There was a significant relationship $(r=0.79, p<0.0005)$ between PS and the REE. The slope of the regression line indicated a net cost of PS of $3.6 \mathrm{kcal} / \mathrm{g}$. We conclude that obesity in children is associated with an absolute increase in whole-body protein turnover consistent with the increased FFM and REE.
So9-16

T-WAVE AMPLITUDE AND HEART RATE VARIABILITY: TWO NON-INVASIVE WINDOWS ON AUTONOMIC CARDIAC REGULATION Validation in cardiac transplant recipients during moderate exercise

Farinelli C.C.J., Ferretti G., Marconi C. ${ }^{*}$, Carù B. ${ }^{*}$, Cerretelii P. and Girardier L. Departmenis of Physiology, CMU, CH-1211 Genève 4, Swizzerland and "ITBA-CNR, Milano, ttaly, and "Department of Cardiology, Rehabilitation Hospital, Tradate, Italy.

The aim of this study was to validale the complementarity of two new non-invasive methods for the assessment of autonomic regulations. Reactions to a moderate exercise ( $75 \mathrm{~W}$ on a cycloergometer) were compared in cardiac transplant recipients (CTR) who modulate their heart rate (HR) by means of circulating catecholamines, their heart being denervated, and in normal subjects where such exercise level doesn't stimulate the sympathetic system. The methods used were: 1) the spectral analysis of $H R$ variability where the high frequency component $(\mathrm{HF}$, above $0.15 \mathrm{~Hz}$, related to the respiratory frequency) is a marker of the vagal activity, the low frequency component ( $L F$; at about $0.1 \mathrm{~Hz}$ ) depends on both sympathetic and parasympathetic activities and the LF/HF ratio indicates changes in the sympatho-vagal balance; and 2) a pure sympathetic index obtained from the amplitude of the T-wave of the ECG (TWA) which is decreased by adrenergic stimulation of the myocardium. Our results indicate that in both groups HR was increased by the exercise. In CTR, a net decrease in TWA $(-39 \pm$ $10 \%, n=6$ ) was measured. Contrasting with this, in normal subjects, no change in TWA $(+2 \pm 12 \%, n=6)$ but a decrease in both HF $(-67 \pm 13 \%, n=7)$ and $L F(-85 \pm 5 \%, n=7)$ without change in $L F / H F$ ratio $(-19 \pm 26 \%, n=7)$ were observed. These results indicate that CTR subjects increase their HR by an adrenergic stimulation, whereas the normal subjects regulate their $\mathrm{HR}$ for such moderate exercise only by reducing their vagal tone.

\section{S09-17}

OXYTOCIN STIMULATES INSULIN SECRETION.

Bobbioni-Harsch E., Frütiger S., Hughes G., Panico M., Morris H., Jeanrenaud B. Laboratoires de Recherches Métaboliques, Geneva, switzerland

Analysis of amino-acid composition, sequence and mass-spectrometry demonstrated the presence of oxytocin (OT) in fractions from hypothalamic and hypophysial extracts, able to stimulate insulin secretion in vitro. Purified natural oT produced a dose-dependent effect on insulin output with a $E D_{50}$ of $39 \mathrm{pM}$, in presence of $5 \mathrm{mM}$ glucose. OT antagonist $(6.6 \mathrm{nM})$ reduced by $70 \%$ the effeects of $66 \mathrm{pM}$ oT. Since oT was demonstrated to stimulate insulin output at physiological concentrations, its potential role as insulin releasing-hormone is proposed.

S09-18

\section{THE "GLUCOSE PARADOX" IN THE ANOXIC AND REOXYGENATED EMBRYONIC MYOCARDIUM Raddatz, E., Tran, L., Rochat, A.-C., Kucera, P. and de Ribaupierre, $Y$}

Institut de Physiologie de l'Université, CH-1005 Lausanne. The hearts of 4-day-old chick embryos explanted in vitro react rapidly, reversibly and reproducibly to anoxia and reoxygenation. This work deals with the effects of exogenous glucose on the mechanical activity of the hearts submitted to strictly controlled normoxia-anoxia-normoxia transitions. The anoxic periods lasted from 10 to 60 seconds. Instantaneous heart rate, amplitude of contraction, velocities of contraction and relaxation of the ventricle wall were determined optically. In presence of 8 and $20 \mathrm{mmol} / 1$ of glucose 1) the arrest of cardiac activity under anoxia was delayed by 60 and $110 \%$, respectively, 2) the duration of the postanoxic cardioplegia was reduced and 3 ) recovery was faster. Paradoxally, these concentrations of glucose induced arrhythmias both during anoxia and reoxygenation. The incidences of such arrhythmias increased with the duration of anoxia. The absence of glucose or blockade of glycolysis suppressed arrhythmias. Image analysis showed that the observed myocardial dysfunctions originated in the atrium and were sometimes associated with disturbances of propagation. 


\section{Regulation of the Cell Cycle}

\section{S10-01}

Okadaic acid a phosphatase inhibitor induces meiotic reinitiation in incompetent oocytes

Gavin A. C.1, de Vantéry C. ${ }^{1}$, Vassalli J. D. ${ }^{2}$ and Schorderet-Slatkine S.1

1 Clinique de Stêrilité et d'Endocrinologie Gynécologique, HCUG, Genève, Suisse; 2 Institut d'Histologie et d'Embryologie, CMU, Genève, Suisse.

The microinjection of okadaic acid into incompetent oocytes leads to the release of the prophase block with entry into $M$-phase. These $\mathrm{G} 2 / \mathrm{M}$-like alterations include chromatin condensation, germinal vesicle breakdown (GVBD), microtubules reorganization and formation of an abnormal (often multipolar) spindle of meiosis I, with chromosomes not aligned on the metaphase plate. The polar body is never extmuded. Moreover okadaic acid microinjection induces the emergence of phosphorylated cytolasmic foci as detected by the monoclonal antibody MPM-2, phosphorylated cytolasmic foci as detected by the monoclonal antibody

known to react with mitotic phosphoproteins associated with centrosomes. The kinetics of GVBD following microinjection is extremely retarded (24-48hrs)
when compared to spontaneous maturation (30man-1hr) and depends on the oocyte diameter. Interestingly, when incompetent oocytes are cultured during $48 \mathrm{hrs}$ and then microinjected with okadaic acid meiosis resumes within a short delay (3-6hrs). MPM-2 epitopes are present on centrosomes only after okadaic acid injection.

Following GVBD one of the step depending on protein synthesis, the expression of tissue type plasminogen activator, is triggered. Indeed OA-injected incompetent ocytes produce small amounts of this activator.

Altogether these results argue for an effect of okadaic acid favoring entry into $\mathrm{M}$ phase including microtubule organization, centrosomes phosphorylation and the recruitment of one of the masked mRNAs ( $\mathrm{PPA}$ ).

\section{S10-02}

HISTONE HI KINASE ACTIVITY, GERMINAL VESICLE BREAKDOW AND M PHASE ENTRY IN

A.-C. Gavin', J.-C. Cavadore ${ }^{2}$ and $S_{j}$ Schorderet-Slatkine'. 'Clinique de

Stérilité, HOG, CH-1211 Genève. ${ }^{2}$ CNRS-INSEFM, Montpellier, france.

Meiotic reinitiation of the mouse oocyte is caracterized by a slow entry into metaphase I, beginning with germinal vesicle breakdown (GVBD) and ending with spindle formation. It is accompanied by a cascade of protein kinases and phosphatases increasing protein phosphorylation. The activation of histone H1 kinase (maturation promoting factor, MPF) and of the $042^{\text {MAPK }}$ have been compared during spontaneous or okadaic acid (OA)-induced meiotic reinitiation. In spontaneously maturing cocytes, histone $\mathrm{H} 1 \mathrm{kinase}$ activity increases before GVBD (2X) and is associated with the disappearance of the upper (tyrosine phosphorylated) migrating form of $p 34^{\text {odc2 }}$. Following GVBD, histone H1 kinase activity culminates (8X) in metaphase 1 . Activation by phosphorylation of p42 MAPK occurs after GVBD. In contrast, no increase of histone $\mathrm{HI}$ kinase is detectable in occytes induced to reinitiate meiosis by a transient exposure to DA, neither before GVBD nor during the following 7 hours of culture. The upper migrating form of $\mathrm{p} 34^{\text {cdc2 }}$ is present for 8 hours. The activation of $\mathrm{p} 42^{\text {MAPK }}$ begins before GVBD. Furthemore, when OA is applied on aocytes microinjected with $\mathrm{p} 13^{\text {suc1. }}$, neither increase of histone $\mathrm{H}$ l kinase activity nor $\mathrm{p} 34^{\mathrm{cdc} 2}$ dephosphorylation are observed although GVBD is induced; $042^{\text {MAPK }}$ is activated. A7together these results suggest that GVBD may or may not be assciated with a detectable activation of histone H1 kinase, depending on the experimental conditions. Activation of $\mathrm{p} 34^{\mathrm{cdc} 2}$ and $\mathrm{p} 42^{\text {MAPK }}$ are separable events. We propose that, in the mouse oocyte, $O A$ might be able to activate an alternative pathway leading to GVBD that is Cdc2-independent and that involves PA2 MAPK activation ensuing MPF-independent phosphorylations.

\section{\$10-03}

\section{Characterization of S. cerevisiae Replication Factor C (RFC)}

\section{Gerhard Cullmann, Karen Fien and Bruce Stillman}

Cold Spring Harbor Laboratory, Cold Spring Harbor, NY 11724, US.A

RFC was originally identified in human cell extracts as one of the cellular factors essential for the replication of SV40 origin-containing DNA in vitro. It is a five subunit protein with one large subunit of $100-140 \mathrm{kDA}$ and four small subunits of $36-40 \mathrm{kDa}$. RFC binds specifically to primertemplate structures at the $3^{\prime}$-end of the primer. Together with proliferating cell nuclear antigen (PCNA) it serves as a primer recognition factor for DNA polymerase $\delta$ and $\varepsilon$.

To show the involvement of RFC in cellular DNA replication we are currently cloning $S$. cerevisiae RFC. The amino acid sequences of a nurnber of tryptic peptides have been obtained. This information was used to amplify parts of the S. cerevisiae genes by PCR and to screen a genomic library with these probes. Three genes were cloned so far. The large subunit, $R F C l$, codes for a $95 \mathrm{kDa}$ polypeptide that is $36 \%$ identical to the large subunit of human RFC (hRFC). The sequences for two of the small subunits were also obtained. $R F C 2$, coding for a $40 \mathrm{kDA}$ polypeptide, is $50 \%$ identical to the hRFC $37 \mathrm{kDA}$ subunit. RFC3, coding for a $38 \mathrm{kDA}$ polypeptide, is $51 \%$ identical to the hRFC $36 \mathrm{kDa}$ subunit. There is also considerable similarity between the different subunits. $R F C I, R F C 2$ and $R F C 3$, as well as the hRFC subunits, have consensus sequences for a ATP/GTP binding site. All three genes are essential in S. cerevisiae.

\section{S10-04}

MITOSIS-SPECIFIC PHOSPHORYLATION OF MIDDLE-T ANTIGEN IS REQUIRED FOR POLYOMAVIRUS-MEDIATED CELL TRANSFORMATION

Pérez, L., Urich, M. and Ballmer-Hofer, K., Friedrich Miescher- Institute, 4002 Basel, Switzerland

Transformation of cells by polyomavirus is mediated by middle- $T$ antigen. This viral protein is able to form complexes with src family tyrosine kinases (e.g. c-Src), phosphatidylinositol-3-kinase (PI 3-K) and phosphatase 2A (PP2A). C-Src associated with middle-T is constitutively dephosphorylated at tyrosione 527 , a site negatively regulating the activity of this enzyme. This results in high tyrosine kinase activity in interphase and mitotic polyoma-transformed cells. Middle-T is transiently hyperphosphorylated during mitosis as reflected by an increase in the apparent $M$ on SDS acrylamide gels. Two putative phosphorylation sites for a cyclin-dependent kinase present in middle- $T$, threonine 160 and threonine 291 , are also phosphorylated by purified $\mathrm{p} 34^{\mathrm{cdc} 2}$ in vitro. We constructed mutant middle-T genes where individual phosphorylation sites were converted to alanine residues. Mutants lacking threonine 160 were still able to associate with all cellular enzymes described above yet defective for cell transformation. Interestingly, the defect of this mutation could be compensated by additional changes in the middle- $T$ protein sequence introduced further downstream in a domain suspected to play a role in the targeting of this protein to intracellular membranes.

\section{S10-05}

ANALYSTS OF GENES REGULATING CYTOKINESIS IN THE FISSION YEAST S. POMBE

Schmidt, S., Fankhauser, C., and viesturs, S. ISREC, 1066 Epalinges, Switzerland

Little is understood of the mechanisms which regulate cytokinesis, or how it is integrated with mitosis. We have cloned a number of genes from the Eission yeast $S$. pombe which are required for the initiation of septation and are characterising them. Defects in the cact and cacl1 genes result in continued nuclear division without cytokinesis, while cdc16 mutants undergo multiple rounds of septum formation without cell cleavage. Our analysis suggests that phosphorylation will play an important role in the regulation of cytokinesis and its integration with mitosis. A functional cdel6 product is required for maintenance of p34cac2 activity in mitosis and the primary sequence of the $c d c 7$ protein indicates that it encodes a protein kinase. Data concerning the role of the cdc7, cdc11 and cdc16 genes will be presented.

S10-06

\section{MUTATTON OF THE AMTNO-TERMINAL PRC PHOSPHORY- LATION SITE ALTERS ACTIVATION OF C-SRC DURING MITOSIS.}

Meili, R. and Ballmer-Hofer, $K$.

Friedrich Miescher Institut, Basel

Tyrosine kinase activity of $\mathrm{c}$-Src is cell cycle regulated. Mutations which interfere with phosphor-ylation at 1527 lead to constitutive activation of C-SrC and transformation of the host cell. We investigated the role that the PKC phosphorylation site plays in the regulation of c-Src in vivo.

The mutant protein ( $12->\mathrm{A})$ shows an altered phosphorylation pattern during mitosis. We mapped these changes by analyzing tryptic digests and further investigated mitotic activation of the mutant protein by in vitro kinase assays. 


\section{S10-07}

CELLULAR DISTRIBUTION OF PP2A DURING THE MAMMALIAN CELI CYCLE

Turowski, P., Fernandez, A.*, Lamb, N.J.C.*, and Hemmings, B.A.

Friedrich Miescher-Institut, 4002 Basel

CRBM, CNRS/INSERM, 34033 Montpellier, France

The protein phosphatase 2A (PP2A) class of phosphoserine/threonine phosphatases comprises a family of holoenzymes where a catalytic subunit (PP2AC) is associated with a "constant" regulatory subunit (PR65) and an additional "variable" regulatory subunit. The latter appears to control substrate specificity and possibly subcellular location of the holoenzyme. It was previously found that the correct functioning of PP2A is crucial for cell cycle progression. We analysed the distribution of PP2AC and PR65 during the cell cycle in synchronised rat fibroblasts. PP2AC was found at constant levels in the cytoplasmic fraction. However, we observed strong fluctuations in the immunofluorescence staining of nuclear PP2Ac at stages corresponding to the $\mathrm{G}_{0} / \mathrm{G}_{1}$ and $\mathrm{G}_{1} / \mathrm{S}$ transitions. In contrast. the distribution of the PR65 protein was found to remain static in both compartments throughout the cell cycle.

\section{S10-08}

\section{EXPRESSION OF MOUSE DNA POLYMERASE $\delta$}

$R$. Hindges, U. Dudler, and U. Hübscher. Institute of Veterinary Biochemistry, University of Zürich, Winterthurerstrasse 190, CH-8057 Zürich, Switzerland

DNA polymerase $\delta$ (pol $\delta$ ) is a two subunit protein with a catalytic subunit of $125 \mathrm{kDa}$ and a smaller subunit of $50 \mathrm{kDa}$ of unknown function. Both the polymerizing and the $3^{\prime}->5^{\prime}$ exonuclease activities reside in the $125 \mathrm{kDa}$ subunit. Sequencing of the the mouse pol $\delta$ catalytic subunit showed that pol $\delta$ is the most conserved replicative pol (Cullmann G., Hindges R., Berchtold M.W. and Hübscher U., GENE, in press). We synthesized PCR primers and cloned three fragments spanning the whole catalytic subunit. The resulting $P C R$ products, called N-term, Middle and C-term have a size of 1600,1570 and $1280 \mathrm{bp}$, respectively. The primer for the $\mathrm{N}$-term and Middle fragments contained a histidin tag in order to purify the recombinant proteins by using a $\mathrm{Ni}$ NTA column. These fragments were cloned into the expression vector pGEMEX 174. Because of the natural termination codon in the C-term fragment, the histidin tag was placed directly in the vector in front of the fusion protein, generating a new vector, pRHHIS. All three fragments were expressed in E.coli. The fusion proteins could be purified in a single step and were used to raise polyclonal antibodies. Finally, the entire pol $\delta$ sequence was joined together and could be expressed. Data on the characterisation of the antibodies and the proteins will be shown.

\section{\$10-09}

\section{Hsp 70 from calf thymus can influence DNA polymera- se under heat shock conditions.}

Alicja Ziemienowicz' ${ }^{1}$, Maciej Zylicz² ${ }^{2}$ Ulrich Hübscher ${ }^{1}$ 1 Department of Veterinary Biochemistry, University of Zürich, Winterthurerstrasse 190,80 57 Zürich, Switzerland 2 Division of Biophysics, Department of Molecular Biology, University of Gdansk, Kladki 24, 80-822 Gdansk, Poland

We have generated antibodies against DnaK protein, the Hsp70 homolog from Escherichia coli, and used them for detecting of $\mathrm{Hsp} 70$ protein during its purification from calf thymus tissue. The apparently purified protein has a molecular weight of $70 \mathrm{kDa}$, and has been recognized by antibodies against DnaK and eucaryotic Hsp72/73. It possesses a very week ATPase activity, which can be stimulated by different poly- and oligopeptide substrates. Results will be presented showing the influence of Hsp70 on DNA polymerase $\varepsilon$ from calf thymus under heat shock conditions.

\section{S10-10}

P53 INHIBITION OF CELLULAR ENZYMES INVOLVED IN DNA METABOLISM IS RELIEVED BY ITS INTERACTION WITH REPLICATION PROTEIN A, Christian Flothi, Elena Ferraril, Anthi Georgaki', Vladimir N. Podust Carol Prives ${ }^{2}$ and Ulxich Hübscher ${ }^{1}$, 1 Department of Veterinary Biochemistry, University of Zürich-Irchel, 8057 zorich; ${ }^{1}$ Department of Biological Sciences, Coumbia University, N.Y. 10027, USA.

p53 is a major tumor suppressor gene which spefically interferes with the onset of $S$ phase. We have therefore examined the possibility that p53 modulates the normal functions of enzymes and proteins directly involved in DNA replication. We have shown that human wtp53 protein binds physically to calf thymus single stranded DNA binding protein $A, R P-A$. We have also found that p53 blocks DNA helicases in a typical strand displacement assay. This may reflect the fact that 053 is able to reanneal complementary DNA single strands since both wt and some mutant forms of 053 share tere tumor supp tumor suppressor activity. To our surptise, among the helicases which were tested we determined that the p53 inhibition could be released by RP-A in the case of cellular but not in the case of viral DNA helicases. Additionally, we found that p53 can partially inhibit DNA polymerase $\delta$ and $\varepsilon$ holoenzymes on singly primed M13 DNA. This inhibition, however, was only evident when $E$, coli SSB was substituted for RP-A. Our dat thus show that 53 in for refect repair. The p53-RP-A interaction may function to protect replication enzymes from inhibition by 053 under certain physiological conditions.

\section{S10.11}

Identification of a vertebrate $c d c 2$ mutant which is unable to complete the G1/S transition.

Nicole_Schmit, and Viesturs Simanis

Chemin Boveresses 155, 1066 Epalinges,.ISREC.

$S$. pombe strains have been constructed which should permit the genetic and molecular analysis of the events which occur in late G1 when cells become committed to the mitotic cell division cycle and the initiation of DNA synthesis. A number of mutants of the chicken $c d c 2$ gene were constructed during the course of analysis of phosphorylation sites some of which lead to the interesting phenotype of cold sensitivity when expressed in a $c d c 2$ null background, when the only source of

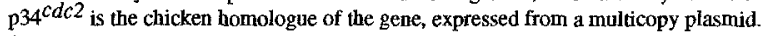
In order to create a genetically tractable strain, the wild-type chicken $c d c 2$ gene and one mutant into the $S$. pombe genome to create a strain in which cell cycle progression is dependent upon the chicken $c d c 2$ gene. Analysis of this strain indicates that the cells block predominantly before replication of DNA. In order to determine whether the cells were arrested before or after the execution of the start control their ability to conjugate at the arrest point was assessed. Consistent with a block before the traverse of start and commitment to S-phase, cells were able to conjugate with high efficiency. Further support for the view that this mutant is defective only for the GI-S transition is provided by the observation that it is able to complement a $c d c 2 A 2 I$ mutation in trans. This mutant is defective only for G2 function and not traverse of start. The double mutant is viable at the restrictive temperature of either of the parents and is no longer cold sensitive. Further work will concentrate on cloning genes involved in the traverse of $G 1$ into $S$ phase in fission yeast.

\section{S10-12}

BINDING OF E-MAP-115 TO MICROTUBULES IS REGULATED BY CELL CYCLE-DEPENDENT PHOSPHORYLATION

D. Masson and T. E. Kreis. Département de Biologie Cellulaire; Sciences III: Université de Genève; $\mathrm{CH}-1211$ Genève 4

E-MAP-115 is a microtubule-associated protein predominantly expressed in cells of epithelial origin (Masson and Kreis, 1993, J. Cell Biol. 123:357) where it is localized on a subset of microtubules. When Caco-2 cells are grown to confluency and become polarized, E-MAP-115 expression levels increase concomitant with a higher microtubule stability. Transfection of fibroblastic cells (Vero), which do not contain any detectable E-MAP-115, with cDNA encoding this protein, results in stabilization of microtubules to nocodazole treatment. These data suggest that E-MAP-115 may be involved in the stabilization and reorganization of microtubules in polarizing epithelial cells. Since E-MAP-115 is a stabilizing protein, its interaction with microtubules should presumably be modified at the onset of mitosis to allow disassembly of the interphase microtubule network and formation of the mitotic spindle. Indeed, immunolabeling for E-MAP-115 varies during mitosis. In early prophase, no E-MAP-115 can be detected on the microtubules of the forming mitotic spindle. The protein is observed on the spindle microtubules of metaphase cells and staining becomes bright on the new interphase microtubules of telophase cells. Analysis of the protein in cells blocked in mitosis by low concentrations of nocodazole indicates that E-MAP-115 is hyperphosphorylated. This hyperphosphorylated form of E-MAP-115 is no longer able to co-sediment with microtubules in in vitro microtubule-binding assays. Phosphopeptide map and phosphoamino acid analysis show the existence of novel phosphorylation sites in E-MAP-115 during mitosis compared to the protein during interphase. We are currently characterizing the kinases involved in the modulation of E-MAP-115 activity. 
S10-13

ENDOGENOUS EXPRESSION OF CALRETININ AND ECTOPIC EXPRESSION OF PARVALBUMIN IN HUMAN TUMORAL CELIS ARE CORRELATED WITH OPPOSITE EFFECTS ON THE CELL CYCLE.

Gotzos, V; Schwaller, B.; Celio, M.R.

Institute of Histology and General Embryology, University; $\mathrm{CH}-1700$ Fribourg

Little is known about the specific functions of calcium-binding proteins in epithelial cells and in particular in tumoral cells of epithelia origin. Calretinin (CR) and parvalbumin (PV) are supposed to be involved in cell proliferation. We observed the endogenous expression of $C R$ in several cell lines from human colon adenocarcinomas, and we obtained stably transfected cells for $\mathrm{PV}$ in a human ovarian adenocarsinoma cell line. We studied the cell cycle in correlation with the endogenous or ectopic expression of $C R$ and $P V$, respectively. $G_{1}$ and mitotic cells are strongly labelled with the CR-antiserum 7696, while PV immunoreactivity is dominan in interphasic, but not in mitotic cells. In CR expressing cells, the cell cycle seems to be normal and the rate of proliferation to be proportional to the percentage of positive cells. The cell cycle is inhibited at mitosis after the addition of $\mathrm{CR}$ antisense oligo nucleotides to the culture medium. The cell cycle is blocked in $G_{1} / S$ and $G_{2}$ in the cells ectopically expressing $P V$.

The results support the hypothesis that these two calcium-binding proteins, $\mathrm{CR}$ and $\mathrm{PV}$, could intervene at different moments and probably with different mechanisms on the mechanism of the cell cycle in the tumoral cells studied.
S10-16

\section{A YEAST GENE HOMOLOGOUS TO THE MAMMALIAN TRANSLATIONALLY CONTROLLED TUMOUR PROTEIN TCTP : GENE DISRUPTION AND EXPRESSION STUDIES.}

Schaller D.., Belet M. ${ }^{*}$, Nazeri M. ${ }^{*}$ Sanchez J.-C.\#, Hochstrasser D.\# and Deshusses J.". "Département de Biochimie, Université de Genève, $\mathrm{CH}-1211$ Genève 4 and \#Clinique Médicale, Département de Médecine, Hôpital cantonal Universitaire de Genève, $\mathrm{CH}-1205$ Genève

The TCTP (P23) is a growth related tumour protein which is expressed under translational control. Homologous acidic proteins have been found in higher plants and in Saccharomyces cerevisiae (YKL312). The striking conservation of this polypeptide between these very divergent organisms suggests some important but still, unknown cellular function. To address this question, a null mutation was constructed in yeast, by deleting almost all the YKL312 structural gene. The deleted strain shows no detectable phenotype. Therefore, we conclude that, in yeast, this gene is dispensable. The protein YKL 312has been overproduced in $E$. coli and injected to rabbits to raise antibodies. We are now characterizing the YKL312 expression at RNA and protein level.

\section{S10-17}

\section{CLONING OF CDNAS ENCODING HAMSTER AND HUMAN CELI CYCLE REGULATORY PROTEINS}

Ellenrieder, C., Forrer, P., Kosovsky, J., Räschle, M., Mueller, R. and Jaussi, R., Institut für Medizinische Radiobiologie, Paul Scherrer Institut \& Universität $\mathrm{zH}, \mathrm{CH}-5232$ Villigen

Radiation therapy of tumors could be improved by influencing cellular radiation sensitivity. Yeast strains with defective cell cycle checkpoint genes (e.g. S.pombe rad1, rad3, rad9) show increased radiation sensitivity. Cross-hybridization of a probe from the radg gene on human mRNA Northern blots yielded a single prominent signal. Experiments to clone the corresponding $4 \mathrm{~kb}$ CDNA are underway. We have cloned a hamster cDNA encoding the homologue of human CDK2. Probably, this cDNA encodes the p40 protein from hamster whose phosphorylation responds to radiation checkpoint signals and who does not bind to p13sucl beads. Treatment of cells with CDK2 antisense oligonucleotides is hoped to modulate the radiation sensitivity.

\section{S10-18}

Phenotypical changes of fibroblasts during in vitro wound healing a confocal laser scanning microscopy (CLSM) investigation of punch wounds in fibroblast populated collagen matrices

Werner Baschong, Rosemarie Sïtterlin, and Ueli Aebi M.E.-Müller-Institut am Biozentrum, Uni Basel, Klingelbergstr. 70, CH4056 Basel

The situation in vivo of dermal fibroblasts embedded within the connective tissue can be emulated in vitro by growing the cells in collagen gels. We have investigated how fibroblasts cultivated within a matrix react upon wounding of this dermal equivalent.

Human skin-derived fibroblasts (KD-cells) growing within attached collagen matrices were monitored over a period of 8 days. Fluorescently labeled cytoskeletal elements (tubulin, vimentin, actin) and fibronectin served as phenotypical markers. The 3D-arrangement of fibroblast microtubules, intermediate filaments and microfilaments, as well as of fibronectin was visualized by CLSM and optical sectioning. Cells at the wound margin, adjacent to the wound, and in non-wounded controls, up to a depth of about $100 \mu \mathrm{m}$ were analyzed.

Upon wounding, i.e. upon excision of a $1 \mathrm{~mm} \varnothing$ "punch biopsy" from the center of the collagen matrix, fibroblasts in the wound region oriented towards the matrix defect. Layer-like structures of increased cell density evolved over time at the wound margin. The tissue defect was covered by fibroblasts, reminescent of the situation in vivo. 
Global yeast genome expression analyzed by 2-D PAGE after TCTP homolog gene (YKL312) disruption.

Sanchez, J.-C. ${ }^{1}$, Appel, R. ${ }^{1}$, Schaller, D.2, Belet, M.2, Muslema, N.2, Deshusses, J.2 and Hochstrasser, D. ${ }^{\dagger}$

1 Clinique Médicale, Medicine department, HCUG, Geneva.

2 Biochemistry department, Geneva University, $\mathrm{CH}-1211-4$.

A translationally controlled tumor protein(TCTP) was identified and microsequenced from a human liver sample. Previous publications described the presence of a gene related to TCTP in plants, mouse erythroleukemia and ascetic tumor, human mammary carcinoma and more recently in the yeast. TCTP has no known function but its high degree of homology from plants to human underlines its probable degree of homology from plants to human underlines its probable
crucial role in cell function. In order to study multifactorial changes in crucial role in cell function. In order to study multifactorial changes in
protein expression as a function of the YKL 312 gene disruption in the yeast, we used high resolution two-dimensional gel electrophoresis combined with an efficient computer system (MELANIE). Two groups of gels $(Y K L 312+(n=10)$ and YKL312 $-(n=10))$ were analyzed, compared and classified using correspondence analysis. There was no significant difference between the two populations of gels according to either qualitative and quantitative spots expression except for two spots which completely disappeared. The first one corresponded to YKL312 gene product. The second one corresponded to a $28 \mathrm{KDa}$ peptide with an isoelectric point of 4.6. This YKL 312 associated peptide still needs to be characterized and linked to the yeast genome.

\section{S10-20}

\section{TRANSLATIONAL CONTROL OF PROTEIN PHOSPHATASE 2A}

Stefaan Wera, Regina E. Mayer-Jaekel, Brian A. Hemmings

Friedrich Miescher Institut, P.O.Box 2543, CH-4002 Basel

Protein phosphatase 2A (PP2A) is one of the major cellular Ser/Thr protein phosphatases. The enzyme exists in several holoenzyme forms, containing a $36 \mathrm{kDa}$ catalytic subunit, a subunit of $65 \mathrm{kDa}$ (PR65) and often other putative regulatory subunits such as PR55. The mRNA's encoding the different subunits show a high degree of conservation in the coding and the non-coding regions. We considured the possibility that the $5^{\prime}$ non-translated end in the different PP2A subunit messengers might regulate translation. I $n$ vitro translation revealed that the $5^{\prime}$ truncated forms of the PR55 $\beta$ and PR65 $\alpha$ mRNA's were translated more efficiently (over 20-fold) than the native messenger. Further experiments showed that truncation of the 5 nontranslated sequence of PR65 allowed overexpression in COS cells. We propose that the $5^{\prime}$ non-coding sequences function to coordinate the levels of the different PP2A subunits in the cell.
Novel Receptor Protein Tyrosine Kinase in Brain Miescher GC, Taylor $V$, Pfarr $S$, Ferracin $F$, Kappos L, Honegger $\mathrm{P}^{*}$, Lassmann $\mathrm{H}^{\#}$ \& Steck AJ. Neurology \& Research Dept. Kantonsspital Basel, *Inst. of Physiol. Lausanne \& \#niv. of Vienna. Demyelinating and neurodegenerative diseases are associated with altered cellular responses including processes mediated by receptor protein tyrosine kinases. Using a PCR CDNA cloning approach we have identified a novel member of the eck/elk/eph gene family in myelinating serum-free brain cell cultures. Alternatively spliced cDNAs encoding complete open reading frames for putative transmembrane proteins have been isolated from both rat and human brain. A recombinant protein derived from the rat cDNA was demonstrated to have protein tyrosine kinase activity. An affinity purified antiserum to this protein was used to identify a glycoprotein of $120 \mathrm{kD}$ molecular weight in brain cultures, and developping and adult brain tissue. The protein expression is most prominent during embryonal development and during the first three weeks of postnatal life. By in-situ mRNA hybridisation the transcripts were found predominantly in restricted neuronal populations. 


\section{Cell-Cell Interactions in Connective Tissues}

\section{$511-01$}

OPTICAL MAPPING OF IMPULSE PROPAGATION IN A CELL CULTURE MODEL OF THE PURKINJE - FIRER - VENTRICULAR JUNCTION

Rohr S., and Salzberg B.M., Dept. of Physiology, University of Bem, Bern, Switzerland and University of Pennsylvania Medical School, Philadelphia, PA, USA. In order to investigate systematically the effects of geometrically defined abrupt tissue expansion on the characteristics of impulse propagation, we constructed expanding cellular structures in vitro using patterned monolayer cultures of neonatal rat heart cells (photolithographically patterned substrates.) The preparations, which consisted of narrow cell sirands ( $40-80 \mu \mathrm{m}$ wide) inserting into large rectangular cell areas (side lengths $>1500 \mu \mathrm{m}$ ), were stained with the voltage-sensitive dye di-8-ANEPPS and were imaged onto a 12 X 12 photodiode matrix array (maximal spatial resolution $15 \mu \mathrm{m}$ $X 15 \mu \mathrm{m}$ in the object plane). While impulse propagation was uniform in linear cell strands (average conduction velocity $0.35 \mathrm{~m} / \mathrm{s}$ ), a transient decrease in conduction velocity in the transitional region was invariably observed in the suddenly expanded structures. This decrease was accompanied by marked distotions of the local action potential shapes due to bidirectional electrotonic interactions across the transition: upstream from the expansion, the repolarization phase was interrupted, a few $\mathrm{ms}$ after its inception, by a small secondary depolarization ("spike and dome" configuration), while, downstream, the action potential upstrokes were preceded by prominent prepotentials. In those cases where conduction failed at the transition, action potential durations upstream were dramatically abbreviated. These findings showed that phenomena similar to those recorded in intact Purkinje-fiber-ventricular preparations can be observed in cell ensembles in vitro having comparable geometries but consisting of cells with uniform active membrane properties.

Supported by Swiss NSF grant 823A-028424 (SR) and USPHS grant NS16824 (BMS).

\section{S11-02}

\section{PURIFICATION AND CHARACTERIZATION OF NATIVE CMP (CARTILAGE MATRIX PROTEIN)}

Nik Hauser and Mats Paulsson, M.E. Mueller Institute for Biomechanics, Murtenstr. 35, 3010 Bern

CMP is a major component of several types of cartilage. It is a noncollagenous glycoprotein with a molecular mass of $148 \mathrm{kDa}$ consisting of three identical subunits. CMP is selectively extracted with EDTA-containing buffer what indicates a divalent cation dependent anchorage of the protein in cartilage matrix. Electron microscopy revealed the presence of three compact globular subdomains and sequence analysis indicated the presence of a coiled-coil $\alpha$-helical assembly domain formed at the C-terminal end of each subunit. The trimeric structure was retained after complete reduction under native conditions which reveals that the subunit structure of CMP is not only stabilized by disulfide bridges but also by the coiled-coil assembly domain. Tissue distribution studies in mouse revealed that CMP is selectively expressed in some but not all cartilages.

\section{S11-03}

EXTRA CEILLUIAR MATRTX REGULATES GENE EXPRESSTON IN MOUSE MAMARY EPITHELIAL CEILS. Schmidhauser C.\#, Casperson G.F.•, Bissell M.J.*. \#Biochemistry I, ETH, 8092 Zürich, Switzerland; -Mol.Biol. Monsanto Res. St.Louis, MO 63198; *Cell \& Mol. Biol. LBL, University of California, Berkeley, CA 94720 .

Extra cellular matrix (EOM) regulates the expression of B-casein in cultured mouse marmary epithelial oells. We have developed a functional mamary epithelial cell strain, which expresses high level of milk proteins, forms alveolar-like structures when plated onto a reconstituted basement membrane and secretes casein unidirectionally into a lumen. We have further shown that ECM-dependent regulation of B-casein occurs mainly at the transcriptional level and that $5^{\prime}$ sequences play an important role in this regulation. We have located a 160bp -transcriptional enhancer (BCE1) within the 5' flanking region of the $B$-casein gene. Using functional assays, we show that BCE1 contains responsive elements for ECM- and prolactin-dependent regulation. BCE1 placed upstream of a truncated inactive B-casein promoter reconstitutes a promoter even more potent than the intact promoter, which contains BCE1 in its natural context more than $1.5 \mathrm{~kb}$ upstream. This small fusion promoter reconstitutes the normal regulation pattern. We show that BCEI mediates EaM dependent regulation even when Iinked to a heterologous viral promoter.
S11-04

\section{Myofibrillogenesis in Cultured Adult Cardiomyocytes}

B.M.Rutishauser, J.M.Messerli, P.Walther*, H.M.Eppenberger and J.C.Perriard

Institute for Cell Biology, ETH-Hönggerberg, 8093 Zürich and *ETHZentrum, 8092 Zürich.

Adult rat cardiomyocytes (ARC) in culture degenerate the myofibrillar apparatus and after attachment to the substrate they grow and reassemble new myofibrils. The appearance of new, well organized myofibrils can be observerd in several distinct regions. They appear close to the membrane proximal to the culture substrate and in the perinuclear region close to the substrate.

Previous studies have shown that embryonic cultured cells assemble new myofibrils which insert in adherens junctions at sites of cell-cell contacts and in sub-sarcolemmal adhesions plaques (SAPs) at sites of cell-substrate contacts. This SAPs are thought to function as the nucleation sites for myofibril formation.

We have investigated the formation of this SAPs in cultured adult rat cardiomyocytes. Using confocal light microscopy we can show that the interaction between myofibrils and membrans occurs close to the membrane proximal to the substrate and that this SAPs are flanking both nascent myofibrils and SFLS, the latter structures being believed to serve as scaffold for myofibrillogenesis. Additionally we have used electron microscopy to investigate the formation of this structures at higher resolution.

S11-05

SELF-RENEWAL PROPERTIES

OF HUMAN MUSCLE SATELLITE CELLS IN VITRO

Baroffio, A. and Bader, C.R. Département de Physiologie, CMU

et Division de Neurophysiologie clinique, HCU, GENEVA

Muscle satellite cells (SC) are quiescent myoblasts, ready to be activated and to regenerate new muscle fibers in case of muscle damage. In vitro, single human muscle $\mathrm{SC}$, cultivated as clones, give rise in proliferating conditions to two subpopulations of cells, cells expressing both $\alpha$-striated muscle actin and desmin ( $\alpha$ $\mathrm{SR}^{+} \mathrm{DSM}^{+}$), and cells expressing desmin alone (DSM${ }^{+}$). In culture conditions promoting differentiation, a clone typically gives rise to a fusing progeny, vielding $\alpha$ $\mathrm{SR}^{+} \mathrm{DSM}^{+}$myotubes, and to non fusing myoblasts (NFMB). The latter are $\mathrm{DSM}^{+}$, a phenotype similar to quiescent SC found in situ.

In order to determine whether NFMB are the in vitro equivalent of in situ quiescent SC, this first generation of NFMB were selectively collected and subcultured. In proliferating conditions, NFMB were able to resume proliferation and to give rise to a progeny with both $\alpha-\mathrm{SR}^{+} \mathrm{DSM}^{+}$and $\mathrm{DSM}^{+}$cells. When cultured in differentiating conditions, NFMB formed $\alpha$-SR ${ }^{+} \mathrm{DSM}^{+}$myotubes, and a new generation of $\mathrm{DSM}^{+} \mathrm{NFMB}$. When NFMB of the second generation were again selectively collected and subcultured, they resumed proliferation and were again able to yield both myotubes and a third generation of NFMB. NFMB of the first generation were also cultivated as clones, and 5 to $25 \%$ of them were able to resume extensive proliferation, yielding in their progeny both $\alpha-\mathrm{SR}^{+} \mathrm{DSM}^{+}$and $\mathrm{DSM}^{+}$cells, which differentiated into myotubes as well as NFMB.

These results strongly suggest that $\mathrm{SC}$ bave the stem cell property of selfrenewal, yielding in their progeny both cells ready to differentiate into muscle and cells similar to themselves.

\section{$\$ 11-06$}

Synthesis and release of an acetylcholine-like compound from human muscle satellite cells.

M. Hamann*, J.P. Ternaux ${ }^{+}$, M.C. Chamoin ${ }^{+}$, P. Portalier ${ }^{+}$, A. Baroffio* and C.R. Bader*. Département de Physiologie*, CMU, 1211 CH-Genève-4 and Unité de Neurocybernétique Cellulaire ${ }^{+}$, UPR 418, 13009 F- Marseille.

Purified satellite cells (SC) were obtained from human skeletal muscle biopsies following cell sorting by flow cytometry. A chemiluminescent assay for acetylcholine (ACh) revealed the presence of 1,3 nmoles/well of 100.000 SC. In clonal cultures of proliferating $\mathrm{SC}$, this intracellular level of $\mathrm{ACh}$ was 0.1 nmoles/well. When cells were cultured in the presence of an esterase inhibitor (10 $\mu \mathrm{M}$ phospholine), the $\mathrm{ACh}$ amount was enhanced 2 fold. Conversely, cultivating the cells in presence of a potent inhibitor of choline acetyl transferase ( $2 \mu \mathrm{M}$ BromoACh), gave a $50 \%$ decrease of $\mathrm{ACh}$ content. Finally, the release of ACh was detected in the supernatant of cultured SC, following a 15 min incubation, and the measured level of ACh was 3 pmoles/well/min

The presence of an ACh-like compound in myogenic cells in both freshly isolated and in proliferating SC suggests that SC may contain ACh in situ. In addition, the fact that $\mathrm{ACh}$ was present in the extracellular medium suggests that $\mathrm{ACh}$ can be released spontaneously by the cultured SC 
S11-07

\section{BINDING OF TYPE XII COLLAGEN TO COLLAGEN TYPE I FIBRILS}

Koch, M., Matthisson, M., and Chiquet, M., Abt. Biophysikalische Chemie, Biozentrum der Universität, CH-4056 Basel

Type XII collagen is an extracellular matrix protein associated with collagen fibrils in vivo. As observed for several other extracellular matrix proteins, the synthesis of type XII collagen by chick fibroblasts cultured in $0.1 \%$ FCS is stimulated by TGF-B1. Two splice variants of type XII collagen are known, with subunits of either $220 \mathrm{kDa}$ or $350 \mathrm{kDa}$. By using antibodies specific for the large $(350 \mathrm{kDa})$ form of type XII collagen, we could show a more restricted distribution of the large variant compared to the smaller form in the embryo. While only the large variant carries chondroitin sulfate, both variants are identical in their collagenous domain. It is thought that via this domain, type XII collagen binds to collagen type I fibrils. We demonstrate here that type XII collagen can bind to collagen I fibrils also in vitro. By neutralizing acid soluble collagen type I, we could coprecipitate type XII collagen together with newly formed collagen type I fibrils. This interaction occurs in physiological saline but is highly salt dependent. In further studies we investigated wether $35 \mathrm{~S}$-methionine labeled type XII collagen treated with chondroitinase $\mathrm{ABC}, \alpha$-chymotrypsin, or collagenase can still be precipitated together with type I fibrils. In addition, we found that type XII collagen also affects the rate of type I collagen fibril formation.

\section{S11-08}

\section{H.U. Keller}

Department of Pathology, University of Bern, $\mathrm{CH}-3010$ Bern

\section{Locomoting blebbing cells}

colchicine $\left(10^{-5} \mathrm{M}\right)$ can induce locomotion associated with blebbing in walker carcinosarcoma cells. Blebs expand at a rate (about $2 \mu \mathrm{m} / \mathrm{sec}$ ) which is much faster than known rates of actin elongation. This suggest that the membrane is directly pushed forward by forces other than actin elongation, possibly by hydrostatic pressure. This interpretation is suggested by the observation that there is significant $\vec{F}$-actin staining all along the cell membrane but not with the cytoplasmatic content the blebs. Blebbing is suppressed by $0.5 \mathrm{M}$ sorbitol. The finding that cytochalasin D suppresses blebbing shows that actin polymerization is nevertheless indixectly instrumental in bleb formation, possibly by generating a high intracellular pressure. A tentative model explaining locomotion of blebbing cells is presented.
S11-09

THE ROLE OF COLONY-STIMULATING FACTOR 1 IN OSTEOCLAST FORMATION

W. Hofstetter, A. Wetterwald, M.G. Cecchini, H. Fleisch and R. Felix

Institute for Pathophysiology, University of Bern, CH-3010 BERN

Osteopetrosis encompasses a family of diseases with different causes and the common phenotype of impaired bone resorption. The osteopetrotic mouse mutant $\mathrm{OD} / \mathrm{OD}$ is deficient in CSF-1, the growth factor for the cells of the mononuclear phagocytic system. The phenotype of $\mathrm{Op} / \mathrm{Op}$ mice is characterized by a low number of peritoneal macrophages and peripheral monocytes, and a virtual absence of
osteoclasts, leading to the osteopetrotic phenotype. Injections of CSF-1 into op/on mice reversed the osteopetrotic phenotype, proving the requirement for CSF-1 during the process of osteoclastogenesis.

By in situ hybridization, expression of CSF-1 was demonstrated during bone development. Osteoclast precursors and mature osteoclasts were identified as putative target cells for the growth factor, since these cells express CSF-1 receptors, which is encoded by the proto-oncogene c-fms. Subsequently, specific binding of CSF-1 to osteoclasts was shown. Expression of c-fms parallels the expression of CSF-1 in bone both in time and place, suggesting a local action of this growth factor in osteoclast formation, but also in the modulation of osteoclastic activity.

Different transcripts, raised by alternative splicing of a commmon nuclear RNA precursor, have been found to encode a secreted and a membrane associated forms of CSF-1. The secreted form can be modified by attachment of a glycosaminoglycan side chain, which serves as an anchor to integrate the peptide into the extracellular matrix. Investigation of the role the different CSF-1 forms play during recruitment of osteoclasts and regulation of their activity will provide further insights into the mechanisms governing these processes. 


\section{Ion Channels}

S12-01

DISTINCT ROLES OF TWO INVARIANT CYSTEINE RESIDUES IN THE POTASSIUM $\left(K^{+}\right)$CHANNEL KV2.1 (DRK1) Zühlke R.D., Zhang H.-J. and Joho R.H., Deptartment of Cell Biology \& Neuroscience, UT Southwestern Medical Center, Dallas, TX 75235-9111, USA.

Comparative sequence alignments of known voltage-gated $\mathrm{K}^{+}$channels revealed two conserved cysteine residues in the putative transmembrane segments S2 and S6. If the cysteines were connected by a disulfide bridge, it would put a structural constraint on possible channel models by placing S2 next to S6. We used site-directed mutagenesis to study systematically the potential roles of these invariant cysteines. Fourteen of 17 substitutions in S2 (C232), and 7 of 19 substitutions in S6 (C393) maintained channel function in Xenopus oocytes microinjected with mutant cRNA. Therefore, the conserved cysteines are not essential for $\mathrm{K}^{+}$channel expression in Xenopus oocytes. However, electrophysiological characterization of the cysteine replacement mutants revealed distinct roles for the two cysteines. Inactivation, deactivation, and ion permeation did not considerably change in $\$ 2$ mutants. In S6, in contrast, cysteine replacement by leucine, asparagine, glycine and valine accelerated inactivation and deactivation kinetics substantially, whereas serine and threonine showed opposite effects. Furthermore, the voltage dependence of deactivation was differently affected. In summary, it appears that the side-chain at position 393 in $\$ 6$ of $\mathrm{Kv} 2.1$ is involved in channel gating by participating in the transitions from the open to the closed and inactivated state of the channel.

(Supported by grants from the NIH and MDA to RHJ, and from the SNF and the Swiss Foundation for Medical-Biological Grants to RDZ.)

\section{S12-02}

CYSTEINE SUBSTITUTION MUTAGENESIS: A TOOL TO PROBE THE MOUTH OF $\mathrm{K}^{+}$CHANNELS BY SULFHYDRYL-SPECIFIC REAGENTS.

Zühlke R.D., Kürz L.L., Zhang H.-J. and Joho R.H., Deptartment of Cell Biology and Neuroscience, UT Southwestern Medical Center, Dallas, TX 75235-9111, USA.

The segment between $\$ 5$ and $\mathrm{S} 6$ of voltage-gated $\mathrm{K}^{+}$channels, called $\mathrm{H} 5$ or $\mathrm{P}$ region, has been implicated to form part of the ion conduction pathway. Little is known about the conformation of this region although various models have been proposed including a $\beta$ barrel-like structure formed by four adjacent antiparallel $\beta$ sheets. To gain insight into the secondary structure of this region, we used cysteine substitution mutagenesis and sulfhydryl-specific, membrane-impermeant reagents to probe the accessibility of amino acid side chains in and around the $\mathrm{P}$ region of $\mathrm{Kv} 2 . \mathrm{I}$ (DRK1). Twenty-eight positions from K356 to T383 were each mutated to a cysteine. After expression of mutant $\mathrm{K}^{+}$ channels in Xenopus oocytes, cysteine side chain accessibilities were probed by superfusion with $\mathrm{CH}_{3} \mathrm{SO}_{2} \mathrm{SCH}_{2} \mathrm{CH}_{2} \mathrm{NMe}_{3}{ }^{+}$(MTSET). MTSET can form a mixed disulfide with an accessible cysteine, and this may lead to current reduction if the covalently modified side chain is in or close to the ion conduction pathway. Thus far, we have identified four mutations that showed $\mathrm{K}^{+}$curnent reduction after superfusion with $3 \mathrm{mM}$ MTSET. The mutants P361C, I379C, Y380C, and K382C showed $87 \%, 99 \%, 39 \%$, and $21 \%$ reduction in current amplitude, respectively. In contrast, mutants S363C, $\mathrm{A} 367 \mathrm{C}, \mathrm{T} 368 \mathrm{C}$ behaved like wild type $\mathrm{Kv} 2.1$ with less than $5 \%$ current reduction. Our results suggest that the side chains of $\mathrm{P} 361,1379$, Y380, and $\mathrm{K} 382$ are directly accessible from the extracellular environment. Taken together, these residues may, therefore, face the lumen of the ion channel pore. Furthermore, the degrees of inhibition are in agreement with a model in which the ion conduction pathway narrows from residue K382 to Y380 to 1379. (Supported by grants from NIH and MDA to RHJ, and from the SNF and the Swiss Foundation for Medical-Biological Grants to RDZ.)

\section{$\$ 12-03$}

CHARACTERIZATION BY SITE-DIRECTED MUTAGENESIS OF A DIVALENT CATION BINDING SITE IN THE VOLTAGEDEPENDENT SODIUM CHALNNEL.

I. Favre, E. Schneeberger and L. Schild.

Institut de Pharmacologie et Toxicologie de 1 'Université, CH-1005 LAUSANNE.

we have investigated whether the external divalent cation block of the voltage-dependent $\mathrm{Na}^{+}$channel ( $\mathrm{Skm} 1$ ) results from pore occlusion. Mutations of the $Y 401$ within the $P$ region (pore region) lead all to functional channels: Y401C, $F, W, A, S, H$ and $D . Y 401 C, Y 401 H$ and Y401D confer a high affinity for $\mathrm{Zn}^{2+} \mathrm{block}$. Y401D exhibits a high sensitivity for both $\mathrm{Zn}^{2}+$ and $\mathrm{Ca}^{2+}$ block. Neither $\mathrm{Zn}^{2+}$ nor $\mathrm{Ca}^{2}+$ did occlude completely the channel pore. Both divalent cations induce a residual subcorductance state. Y401H shows a high affinity for porton block. Similarily to the block by $\mathrm{Zn}^{2+}$ and $\mathrm{Ca}^{2+}$, protons only partially block the channel. The ability for all mutated channels to conduct $\mathrm{Na}^{+}$current, and the partial block induced by $\mathrm{Zn}^{2}+\mathrm{Ca}^{2}+$ or $\mathrm{H}^{+}$ indicate that $Y 401$ is not located deep in the pore but rather in the extracellular mouth of the channel.
S12-04

Analysis of BSF1, a Novel DNA Binding Protein Possibly Involved in Cell Type-Specific GABA A-Receptor Gene Expression

Motejlek K., Häuselmann R., and Lüscher B.; Pharmakologisches Institut der Universität Zürich, Winterthurerstr. 190, CH-8057 Zürich

Comparison of $5^{\prime}$ flanking sequences of three GABAA-receptor subunit genes $(\alpha 1, \gamma 2, \delta)$ revealed a novel conserved purine sequence element with the consensus sequence GAGAGGGGAGAGGA GAGAG(GG/AA)G. This element is present once each in the $\alpha 1$ and $\gamma 2$ subunit gene promoters and in seven tandem copies in the $\delta$ gene promoter. A novel DNA binding activity (BSF1) was identified that binds to various versions of this purine element. BSF1 was found only in brain but not in any other tissues tested. Furthermore, the factor is distinct of BETA, another brain-specific DNA binding protein with a purine-rich DNA recognition sequence. The expression of BSF1 during differentiation of cerebellar granule cells in vitro correlates with the expression of GABAA-receptor subunit genes. This correlation suggests a role for BSF1 as a transcriptional activator of neuronal genes. Therefore, BSF1 may be important for cell typespecific regulation of GABAA-receptor gene expression. We also found that BSF1 binds to wheat germ agglutinin, suggesting that this protein is glycosilated. Taking advantage of this property, we are in the course of purifying BSF1. The question, whether BSFI is indeed a transcription factor is being investigated in vivo and in vitro.

\section{S12-05}

THE USE OF SELF-MANUFACTURED $\mathrm{Mg}^{2+}$ MACROELECTRODES TO MEASURE THE Mg-ATP DISSOCIATTON CONSTANT

A. Truttmann, W. Zhang, D. Lüthi \& J. A. S. McGuigan

Physiologisches Institut, Bühlplatz 5, 3012 Bern

To produce a simple method of estimating the free magnesium concentration $\left(\left[\mathrm{Mg}^{2+}\right]\right)$ in solution, we have manufactured dip cast $\mathrm{Mg}^{2+}$ macroelectrodes using the neutral $\mathrm{Mg}$ carrier ETH 7025. We have used the electrodes to measure the dissociation constant $\left(\mathrm{K}_{\mathrm{d}}\right)$ for MgATP in a background solutions mimicking the intracellular milieu. For the measurement of the Mg ATP dissociation constant, the background solution contained $2 \mathrm{mmol} / \mathrm{l} \mathrm{Na} \mathrm{A}_{2} \mathrm{ATP}$. This solution was titrated with $\mathrm{MgCl}_{2}$ and the changes in $\left[\mathrm{Mg}^{2+}\right]$ above $0.05 \mathrm{mmol} / \mathrm{h}$ monitored with the macroelectrode. During the titration the $\mathrm{pH}$ was maintained constant. Fitting such titration curves with the standard hyperbolic binding equation, after correction for zero drift, gave the following mean+SD values for the $\mathrm{K}_{\mathrm{d}}\left(\mu \mathrm{mol} / \mathrm{l}\right.$ at $\left.37^{\circ} \mathrm{C}\right)$; $\mathrm{pH} 6.4,103.3 \pm 25.3(\mathrm{n}=9)$; $\mathrm{pH} 7,59.7 \pm 27.4$ $(n=6)$ and $\mathrm{pH} 7.4,30.8 \pm 15.4(\mathrm{n}=7)$. Elevation of the $\left[\mathrm{Na}^{+}\right]$to $50 \mathrm{mmol} / \mathrm{h}$ and reducing the $\left[K^{+}\right]$to $100 \mathrm{mmo} / \Omega$ was without effect at $\mathrm{pH} 6.4(\mathrm{n}=6)$. Reducing the temperature to $25^{\circ} \mathrm{C}$ increased the $K_{d}$ at 7.4 to $49.0 \pm 21.6(n=5)$,

$\mathrm{Mg}^{2+}$ macroelectrodes provide an easy way of measuring $\left[\mathrm{Mg}^{2+}\right]$ in solution. $\left[\mathrm{Mg}^{2+}\right]$ can also be measured in a background solution containing $1 \mathrm{mmol} / \mathrm{h}$ $\mathrm{Ca}$, although the electrode response is reduced. Interference by protein to date prevents their use in plasma.

\subsection{6}

Progesterone modulates the activity of glycine receptor expressed in colliculli neurons of neonatal rats.

Maury, K. \& Bertrand, D.

Dpt of Physiology, CMU, 1211 Geneva 11.

Steroids are potent narcotics and have been shown to act on ligand-gated channels activated by GABA or nicotine. However, little is known about their possible actions on other receptor types. For instance, while it was demonstrated that progesterone inhibits glycine receptors expressed by spinal cord neurons isolated from chick embryos, nothing has yet been reported for brain receptors.

In this study, we have determined the properties of glycine channels expressed by brain neurons of neonatal rats using the whole-cell voltage-clamp technique. In inferior colliculi neurons, glycine elicited little-desensitizing inward currents which reversed around $-40 \mathrm{mV}$. These currents were blocked by strychnine in the nanomolar range. Surprisingly, however, we found that progesterone, in the micromolar range, potentiated the glycine evoked currents. These results, contrasting those obtained in chick spinal cord neurons, suggest that progesterone enhances or antagonizes glycine currents depending on the subtype of the receptor. 
S12-07

\section{Electrophysiological Properties of Outer Hair Cell from Guinea-Pig Cochlea}

Hai-Yan HU Daniel BERTRAND

Department of Physiology, CMU, ch-1211 Geneve 4, Switzerland

The mammalian auditory organ (organ of Corti) relies on two groups of sensory cell for its normal hearing: Inner Hair Cell \& Outer Hair Cell. IHCs are mainly innervated by afferent fibers while the OHCs essentially receive efferent fibers. The OHCs possess unique electromotile properties which are thought to enhance the motion the basilar membrane and to refine the mapping of the sound frequency. We found, using whole cell recordings, that $5-10 \%$ of the freshly dissociated OHCs displays fast inward currents. The magnitude of peak currents which can be as large as $2 \mathrm{nA}$ vary from cell to cell. Further experiments have revealed that this current is sensitive to TTX and strongly reduced when extracellular sodium is replaced by choline. Its kinetic is relatively slow compared to that of sodium current of typeI spiral ganglion cell, and has a more negative inactivation. Other voltage activated currents and ligand-gated currents are under inverstigation. These results will provide further insights in the hearing transduction mechanism.

\section{$512-08$}

EXPRESSION OF THE KRINGLE 1 DOMAIN OF HUMAN PLASMINOGEN AND ANALYSIS OF ITS BINDING AFFINITY FOR $\omega$-AMINO ACIDS

Marti, D., Schaller, J., Rickli, E.E., Institute of Biochemistry, University of Berne, Switzerland

The fragment $\mathrm{K} 77 \mathrm{M} / \mathrm{KVYLSE}\left[\mathrm{K} 1_{\mathrm{HPg}}\right] \mathrm{EE}$ containing the entire kringle 1 domain (K1) of human plasminogen $(\mathrm{HPg})$ was expressed in Escherichia coli as a non fusion protein using an expression vector which carries the phage T7 promotor $\phi 10$. For refolding the recombinant kringle 1 domain ( $r-K 1)$ was incubated in a denaturing and reducing environment followed by non-denaturing and oxidising conditions. The isolation was achieved by affinity chromatography on lysine Bio-Gel. Final purification was performed on a Mono $Q$ anion exchange column. r-K1 was characterized by amino acid composition, amino acid sequence analysis and determination of the molecular mass. The correct arrangement of the disulfide bonds was verified by sequence analysis of the corresponding subtilisin fragments and the accurate folding of the kringle was checked by high resolution ${ }^{1} \mathrm{H}$ NMR spectroscopy. The dissociation constant for 6-aminohexanoic acid determined by intrinsic fluorescence titration amounts to $13 \mu \mathrm{M}$ and is in good agreement with the value assigned by equilibrium dialysis.

\section{S12-09}

PROTEIN KINASE C TRANSIENTLY ACTIVATES HETEROMERIC NMDA RECEPTOR CHANNELS INDEPENDENT OF THE PHOSPHORYLATABLE C-TERMINAL SPLICE DOMAIN AND OF CONSENSUS PHOSPHORYLATION STTES

S.Kellenberger, R.Baur, P.Malherbe*, E.Sigel. Institut of Pharmacology, University of Bern, *Pharma, Preclinical Research, F.Hoffmann-La Roche Ltd., Basel.

We have expressed dual subunit combinations of isoforms of the $\mathrm{N}$-methyl-D-aspartate (NMDA) receptor, NR1A-NR2A and NR1C-NR2A, in Xenopus oocytes. We show that both forms of the receptor are stereo-specifically activated by low concentrations $(10 \mathrm{nM})$ of $4 \beta$-phorbol 12 -myristate 13-acetate, known to activate protein kinase C (PKC). Activation is transient and rapidly decreases after reaching a maximum after about 10 min, even in the continuous presence of phorbol ester. $2 \mu \mathrm{M}$ oleoylacetylglycerol had similar consequences. NR 1C differs from NR1A by a deletion of 37 amino acids that include four consensus phosphorylation sites for PKC. The corresponding peptide has been shown to become phosphorylated upon activation of PKC in neurons (Tingley et al., Nature 364, 70 (1993)). Activity of NR1A-NR2A and NRIC-NR2A receptors could be stimulated 3.5- and 7-fold, respectively. By site specific mutagenesis, we removed 3 consensus, phosphorylation sites for PKC in NRIC and 1 in NR2A. Coexpression of the mutant subunits showed a similar response to phorbol esters as wild type receptors. Our data indicate that neither the predicted consensus phosphorylation sites between transmembrane sequences M3 and M4, nor the phosphorylatable C-terminal splice domain is essential for the modulation of NMDA receptors by PKC.
S12-10

GLFCTRICAL PROPIRTIIS OF HOMOTYPIC AND HWTIROTYPIC GAP

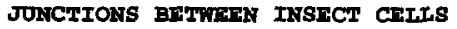

Bukauskas, F.F. and Weingart, $R$

Dept. of Physiology, University of Bern, CH-3012 Eern The formation and the properties of homotypic and heterotypic gap junctions were studied using two types of insect cells, $\mathrm{C} 6 / 36$ and SF 9 . Two single cells were pushed against each other and the subsequent de novo formation of gap junction channels was assessed by means of the dual voltage-clamp method (Bukauskas and Weingart: Pflïg. Arch. 423:152, 1993). Channel formation, homotypic or heterotypic, started within $10-$ 15 min after physical contact. The conductance of a fully open channel (g, (main state)), was 224 pS (SF9-SF9) $303 \mathrm{ps}(\mathrm{SF} 9-\mathrm{C} 6 / 36)$ and $375 \mathrm{ps}(\mathrm{C3} / 36-\mathrm{C} 6 / 36)$. The channels yielded a residual conductance ( $g_{1}$ (residual)) about $1 / 5-1 / 6$ of $g_{\text {g }}$ (main state). In each preparation, the gap junction conductance $\left(g_{f}\right)$ was sensitive to $V$, (junctional voltage) and $V_{m}$ (membrane potential). The relationship $g_{\text {f }}$ (steady state) vs. $V_{j}$ was bell-shaped.
It was symmetrical for homotypic junctions. The $V_{J}$-sensitivity was less pronounced in SF' 9 cells. Hence, the relationship for heterotypic junctions was asymmetrical. AII pairs examined revealed a s shaped relationship between $g_{j}(s s)$ and $V_{m}$ which was virtually superimposable ( $g,(s s)$ declined upon depolarization). Each channel contains a $V_{m}$-gate and two $V_{y}$-gates. The $V_{j}$-gates are operated independently. They close when their intracellular aspect is made positive.

\section{S12-11}

CARDIAC GAP JUNCTIONS : THE ASYMARTR OF V,-GATING DERENDS ON THE PULSE PROTOCOI.

Banach, $K$. ${ }^{*}$ Meda, $P$. and Weingart, $R$.

Dept. of Physiology, University of Bern, CH-3012 Bern, *Dept. of Morphology, University of Geneva, $\mathrm{CH}-121$ Geneva

We used a communication deficient cell Iine (rat islet tumor) after transfection with a cDNA encoding for Cx43, the major cardiac gap jurction protein. Experiments were performed on cell pairs by means of the dual voltage-clamp method. Voltage gradients across the gap junction $\left(V_{f}\right)$ were established and junctional currents $\left(I_{1}\right)$ measured $\left(V_{3}=V_{2}-V_{1} ; V_{1}, V_{2}:\right.$ membrane
potential of cell 1 , cell 2$)$. Small $V_{1} s$ (<50 mV) gave rise to constant $I, s ;$ large $V_{j} s \quad(>50 \mathrm{mV})$ produced $I_{j} s$ exhibiting a time- and voltage-dependence sensitive to the pulse protocol. In case of a non-symmetrical protocol ( $V_{1}$ was stepped, $V_{2}$ maintained), the plot of normalized conductance $\left(g_{f}(s s) / g_{\text {, }}\right.$ (inst) $)$ vs. $V_{y}$ revealed a bellshaped curve compatible with a two-state Boltzmann process. Half maximal inactivation was reached at $-77 \mathrm{mV}$ and $+66 \mathrm{mV}$, implying an asymmetrical gating behavior. In case of an asymmetrical protocol ( $V_{1}$ and $V_{2}$ was stepped simultaneous, but in opposite direction), the $V_{\text {, }}$-dependent asymetry disappeared (half maximal inactivation at $-59 \mathrm{mV}$ and $+60 \mathrm{mV})$. The asymmetry in g, $-\mathrm{gat}$ ing, attributable to the pulse protocol, was also reflected in the time constants of $I_{\text {f }}$ inactivation $\left(\tau_{1}\right)$.

\section{S12-12}

MMDA RECEPTOR FXPRESSION IN CEREBELLAR GRANULE CELLS Villa, $\mathbf{M}^{*}$, Resink, $\mathrm{A}^{+}, \mathrm{Möhlex}, \mathrm{H}^{*}$, and Balázs, $\mathrm{R}^{+}$ "Institute of Pharmacology, ETH and university of Zurich, Winterthurerstrasse 190, CH-8057 zurich, Switzerland + Netherlands Institute for brain research, Meibergdreef 33, 1105 A2 Amsterdam zo, The Netherlands.

Cerebellar granule cell cultures were used to study the regulation of the NMDA receptor expression. We have shown previously that chronic membrane depolarisation $125 \mathrm{mM} \mathrm{K}^{+}$) or treatment with NMDA (100 $\mu$ M) promotes the functional expression of NMDA receptors, as assessed by NMDA-evoked ${ }^{45} \mathrm{Ca}^{2}+$ influx. We have now developed a RNase protection procedure for the quantitative determination of the mRNA levels of the NMDA receptor subunits known so far (NR-I, 2A, 2B, 2C, 2D). The growth conditions mentioned above failed to alter the mRNA levels of the different subunits. At the protein level, NMDA receptors were evaluated quantitatively by the labeling pattern obtained by the new photoaffinity ligand ${ }^{125}$ I-CGP55802A The intensity of the photolabeled bands, thought to represent NR-1 and NR-2A subunits, was increased by treatment with high $\mathrm{K}^{+}$or NMDA compared with control cultures. This result suggest an increase in receptor protein by high $\mathrm{K}^{+}$or NMDA treatment. Thus, posttranscriptional mechanisms seem to play an essential role in the modulation of the NMDA receptor activity. 
$\mathrm{S} 12-13$

ANALYSIS OF THE ERITHELIAL SODIUM CHANNEL (arENAC) IN TRANSGENIC MICE

Hummler, E. 1, Verdumo, C.1, Beermann, F. ${ }^{2}$ and Rossier, B.1 Institute of Pharmacology and Toxicology ${ }^{1}$, Lausanne; Swiss Institute for Experimental Cancex Research ${ }^{2}$ (ISREC), Epalinges, Switzerland

The gene encoding the pore-forming subunit of the rat epithelial $\mathrm{Na}^{+}$channel ( $\alpha$ rENac) was cloned recently and shown to belong to a novel gene family coding for cation channels (Nature 361. p467-470 (1993). Transport of $\mathrm{Na}^{+}$ions through these channels is the rate-limiting step of $\mathrm{Na}^{+}$absorption and thereby controls osmotic balance of body fluids and secretions. In order to study the implication of $\alpha=E N a c$ in regulating sodium balance, blood volume and blood pressure and therefore its involvement in hypertension, we expressed arENac under the control of the human CMV promoter in transgenic mice. Several lines of mice showing expression in different tissues were obtained. Progress in the analysis of these mice will be discussed.

\section{S12-14}

ACTION OF A METABOTROPIC GLUTAMATE RECEPTOR AGONIST IN THE LATERAL SEPTUM OF THE RAT: A WHOLECELL PATCH-CLAMP STUDY.

Metzger D., Dreifuss J.J. and Raggenbass M., Département de Physiologie, Centre Médical Universitaire, CH-1211 Genève 4

Activation of metabotropic glutamate receptors increases the excitability of neurones in the lateral septum of the rat. To elucidate the mechanism(s) of this action, we used whole-cell patch-clamp recordings from coronal brain slices of young animals. In voltageclamped cells, the selective agonist (IS,3R)-ACPD, at 1-50 $\mathrm{M}$, had the following effects. a) It evoked a sustained inward current, which was resistant to TTX and to cadmium and which persisted in neurones loaded with BAPTA, a calcium chelator. This current displayed inward rectification and was reduced, or suppressed, when extracellular sodium was partially replaced with $\mathrm{N}$-methyl-D-glucamine. b) It elicited a TTX-insensitive, voltage-dependent inward current, which activated at $-50 /-40 \mathrm{mV}$ and which reversed at aound $0 \mathrm{mV}$. This current was suppressed in a low-calcium/high-magnesium perfusion solution and was undetectable in the presence of intracellular BAPTA. We suggest that ACPD exerts a dual action on lateral septal neurones. It causes a steady depolarization by generating a sodium-dependent current and it triggers transient plateau potentials by inducing a calcium-dependent cationic current. Interaction between these currents results in a powerful, self-reinforcing neuronal excitation.

\section{S12-15}

WHOLE-CELL NMDA CURRENT IN SUPRACHIASMATIC NEURONS AND ITS MODULATION BY EXTRACELLULAR CALCIUM.

Alberi S., Dubois-Dauphin M., Dreifuss J.J. and Raggenbass M., Département de Physiologie, Centre Médical Universitaire, $\mathrm{CH}-1211$ Genève 4.

We have used whole-cell patch-clamp recordings and hypothalamic slices in order to characterize the effect of $\mathrm{N}$-methyl-D-aspartate (NMDA) on suprachiasmatic neurones. In cells clamped at or near their resting membrane potential, NMDA $(50-100 \mu \mathrm{M})$ generated an inward current of 10-60 pA, which was insensitive to TTX and which reversed at about $0 \mathrm{mV}$. The NMDA current-voltage (I-V) relation contained a region of negative slope conductance. The NMDA current was reduced or suppressed by $\mathrm{D}(-)-2$-amino-5-phosphonopentanoic acid (D-AP5) or by MK-801. It was potentiated by reducing the extracellular magnesium concentration from 1 to $0.01 \mathrm{mM}$, or by adding glycine ( 10 $\mu \mathrm{M})$ to the perfusion solution. In a majority of neurones, lowering the extracellular calcium concentration from 2 to $0.01 \mathrm{mM}$ caused a 1.5 to 4-fold enhancement of the NMDA current. I-V relations indicate that in the low-calcium solution, the region of negative slope conductance was attenuated. This effect was due to extracellular calcium, since it persisted in neurones loaded with the calcium chelator BAPTA. We conclude that NMDA channels present in suprachiasmatic neurones may be modulated by extracellular calcium.
S12-16

DIFFERENTIAL MODULATION OF VOLTAGE-GATED Ca ${ }^{2+} \mathrm{AND} \mathrm{K}^{+}$ CHANNELS BY PROTEIN KINASE C ACTIVATORS AND PHOSPHATASE INHIBITORS IN PC12 CELLS

A. Bouron, B.F.X. Reber and H. Reuter. Pharmakologisches Institut der Universität Bern, 3010 Bern.

We have investigated the effects of protein kinase $C$ (PKC) activators ( $4 \beta$ PMA, OAG) and of phosphatase inhibitors (okadaic acid, calyculin A) on voltage-gated $\mathrm{Ca}^{2+}$ and $\mathrm{K}^{+}$channels in NGF-differentiated PC12 cells. Whole-cell $\mathrm{Ba}^{2+}$ and $\mathrm{K}^{+}$currents were recorded with the patch-clamp technique. By using specific $\mathrm{Ca}^{2+}$ channel blockers ( $\omega$-conotoxin $(\mathrm{CgTX})$, isradipine) we found 3 types of $\mathrm{Ba}^{2+}$ currents $\left(\mathrm{I}_{\mathrm{Ba}}\right)$; a) a $\omega-\mathrm{CgTX}$-sensitive $\mathrm{I}_{\mathrm{Ba}}$; b) an isradipine-sensitive $\mathrm{I}_{\mathrm{Ba}}$; and c) a $\omega-\mathrm{CgTX}$ plus isradipineresistant $\mathrm{I}_{\mathrm{Ba}}$. 4 4 -PMA and $\mathrm{OAG}$ specifically down-modulated the isradipine-sensitive $\mathrm{I}_{\mathrm{Ba}}$. The inhibition of $\mathrm{I}_{\mathrm{Ba}}$ was prevented by staurosporine and PKC (19-31) (2 PK inhibitors). The delayed rectifier $\mathrm{K}^{+}$ current was unaffected by PKC activators. Applied extemally, okadaic acid and calyculin $A$ inhibited the total $I_{B a}$ by affecting several components of the $\mathrm{Ba}^{2+}$ currents. However, the $\omega-\mathrm{CgTX}$ plus isradipine-resistant $\mathrm{I}_{\mathrm{Ba}}$ was unaffected by okadaic acid. In conclusion, our results suggest a differential modulation of voltage-gated $\mathrm{Ca}^{2+}$ and $\mathrm{K}^{+}$channels by the $\mathrm{PKC}$ signalling pathway in NGF-differentiated PC 12 cells.

\section{S12-17}

AGRIN ISOFORMS SYNTHESIZED BY MOTOR NEURONS ARE HIGHLY ACTIVE IN ACHR-AGGREGATION

Gesemann, M, Denzer A.J., and Rüegg, M.A., Dept. of Pharmacology, Biozentrum, University of Basel, 4056 Basel

Agrin, a protein isolated from basal lamina extracts of the electric organ of the marine ray, is thought to mediate the motor neuron-induced aggregation of acetylcholine receptors (AChRs) in muscle fibers at their neuromuscular junction. Recent cloning in the marine ray, rat and chick has revealed that agrin can be alternatively spliced at two sites in its C-terminal half. Site A encodes either 0 or 4 amino acids and site $B$ encodes either $0,8,11$ or $19(8+11)$ amino acids. Studies of agrin mRNA expression indicate that early in synaptogenesis, chick motor neurons contain high levels of A4B19. In contrast, non-neuronal cells and muscle cells contain $\mathrm{A} 4 \mathrm{BO}$ and $\mathrm{AOBO}$ mRNA. In the adult ray, electric lobe motor neurons that innervate the electric organ contain A4B8, whereas agrin mRNA in the electric organ again lacks both sites (McMahan et al. (1992), Curr. Op. Cell Biol. 4:869-874). To investigate the functional properties of agrin isoforms in more detail, we have now compared their specific activity to aggregate AChRs on cultured chick myotubes. Heterologous expression of the C-terminal half in COS-7 and 293 cells revealed that chick agrin isoforms $A 4 B 8$ and $A 4 B 19$ were highly active, while A4B11 was up to 40 fold lower in activity. No activity could be detected for the $\mathrm{A} 4 \mathrm{BO}$ and $\mathrm{A} 0 \mathrm{~B} 0$ isoforms. In agreement with these findings the A4B8 isoform of the marine ray also showed high AChR-clustering activity. Therefore, we conclude that motor neurons throughout development synthesize highly active agrin isoforms while the postsynaptic target cells do not play a primary inductive role in the formation of synapses.

\section{S12-18}

SYNAPTIC BASAL LAMINA MAINTAINS AChR $\varepsilon$-SUBUNIT GENE EXPRESSION AT THE NEUROMUSCULAR JUNCTION. Herczeg, A., Ruegg, M.A.*, Iichtsteiner, M. and Brenner, H.R., Physiolog. Institut, and Abt. Pharmakologie*, Biozentrum, Basel.

During innervation of skeletal muscle, the myonuclei underlying the synapse begin to express acetylcholine receptor (AChR) $\varepsilon$-subunit gene and they remain activated after the nerve is removed. our experiments show that this is due to a factor in the symaptic portion of the muscle fibre basal lamina (BL). one candidate for this factor is agrin, which regulates AChR clustering in the synaptic membrane: 1) Unlike in untreated muscle, the level of $\varepsilon$-mRNA at synapses was strongly reduced in cultured rat muscle Eibres after proteolysis of synaptic BL. 2) Conversely, when rat myotubes were cultured on $\mathrm{BI}$ isolated from adult muscle, they preferentially expressed AChR accumulations and $\varepsilon-m$-mNA at sites where they contacted the synaptic portions of the isolated BL. 3) when various substrates were impregnated locally with agrin A 4 B19, an isoform expressed by motor neurones in fetal spinal cord, it locally induced in the myotubes the expression of $\varepsilon-m R N A$. 
S12-19

An increase of functional nicotinic acetylcholine receptors precedes the fusion of cultured human muscle satellite cells. R. M. Krause, C.-R. Bader and L. Bernheim.

Department of Physiology and Division of Clinical Neurophysiology, University Medical Center, 1211 Geneva 4, Switzerland

Nicotinic acetylcholine receptors (nAChR) are expressed on embryonic myoblast and ACh may play a role in mechanism of fusion. Our study focuses on the appearance of functional nAChR in freshly isolated and cultured satellite cells (SC) from normal human muscle biopsies. SC can be conditioned to either proliferate or fuse to form myotubes depending upon culture media. Presence of ACh-activated current was investigated using the whole-cell and single-channel patch-clamp technique. In freshly isolated SC (whose properties should be close to the quiescent in vivo SC) no $n A C h R$ were observed $(n=16)$. In the proliferating state, $54 \%(n=35)$ of the satellite cells (which are also called myoblasts) displayed a small $\mathrm{ACh}$-activated current $(10$ $\mathrm{pA} / \mathrm{pF}$ ) and, as expected, after fusion of satellite cells into myotubes, $100 \%$ of the cells displayed an $\mathrm{ACh}$-activated current $(\mathrm{n}=6 ; 26 \mathrm{pA} / \mathrm{pF})$. To examine, whether the increased expression of $\mathrm{nAChR}$ precedes $\mathrm{SC}$ fusion, $\mathrm{SC}$ were cultured in a medium which promotes fusion, but were prevented from fusing by keeping them at a low density. In this culture condition, $93 \%$ of the $\mathrm{SC}(\mathrm{n}=15)$ displayed an $\mathrm{ACh}$-activated current and, in addition, these cells expressed a 4 times higher ACh-current density $(40 \mathrm{pA} / \mathrm{pF})$ than the proliferating $\mathrm{SC}$. We also observed that, in high density cultures, a small population of SC do not fuse even after 3 months in the medium promoting fusion. These non-fusing myoblasts (NFMB) had no nAChR. Our results suggest that the appearance of $n \mathrm{AChR}$ may be related to the process of $\mathrm{SC}$ fusion as i) quiescent SC in vivo do not express $\mathrm{nAChR}$, ii) $\mathrm{nAChR}$ expression increases in conditions promoting fusion and iii) no $A C h R$ were observed in NFMB. The latter cells may be equivalent of quiescent $\mathrm{SC}$ in vivo.

\section{S12-20}

CLONING AND FUNCTIONAL ANALYSIS OE THE AMILORIDE-SENSITIVE EPITHELIAL SODIUM CHANNEL OE X.LEAVIS

Alessandro Puoti, Cecilia Canessa and Bernard Rossier

Institute of Pharmacology and Toxicology,

Lausanne.

Sodium readsorption in epithelia such as distal colon, lung and the distal part of the renal tubule is achieved by a recently cloned amiloride-sensitive sodium channel (Nature 361 , 467-470). In rat colon, this channel is comprised of three distinct, yet homologous subunits. Using reverse polymerase chain amplification, we have cloned this epithelial. sodium channel from the $A 6$ cell line, which was derived from Xenopus leavis kidney. This will allow us to investigate the regulation of the transcription of this channel in response to such hormones as aldosterone and vasopressin. The structural and functional aspects of this sodium channel in Xenopus in comparison to the rat homolog are now under investigation.

\section{$512-21$}

\section{SUBUNIT COMPOSITION OF NEURONAL NICOTINIC ACETYLCHOLINE RECEPTORS DETERMINES THEIR SENSITIVITY TO CHOLINERGIC ANTAGONISTS}

Cachelin, A.B. \& Rust, G., Pharmakologisches Institut der Universität Bern, 3010 Bern

We have investigated the sensitivity of neuronal nicotinic acetylcholine receptors (nAChRs) of known subunit composition to various cholinergic antagonists. Neuronal nAChRs were expressed in Xenopus oocytes after injection of pairwise combinations of $\alpha 2$ or $\alpha 3$ with either $\beta 2$ or $\beta 4$ cRNAs. The two-electrodes voltage-clamp technique was used to measure currents induced by rapid application of low concentrations of acetylcholine $(\mathrm{ACh})$ together with increasing concentrations of antagonists. The response of $\alpha 3 \beta 4$ neuronal nAChRs to ACh was halfmaximally inhibited by following antagonist concentrations (IC50): hexamethonium $(0.3 \mu \mathrm{M})$, mecamylamine $(0.2 \mu \mathrm{M})$, pentolinium $(0.2 \mu \mathrm{M})$ and trimetaphan $(1.2 \mu \mathrm{M})$. With $\alpha 3 \beta 2 \mathrm{nAChRs}$ the IC50s of the same antagonists were about 10 times higher. Finally, $(+)$-tubocurarine was a conventional competitive inhibitor of $\mathrm{ACh}$ in $\alpha 3 \beta 2$ and $\alpha 2 \beta 2$ nAChRs. In contrast, low concentrations of $(+)$-tubocurarine increased the response to low ACh concentrations in $\alpha 3 \beta 4$ and $\alpha 2 \beta 4$ nAChRs. These results further underline the importance of $\beta$ subunits in determining the functional properties of neuronal nAChRs.

Supported by the Hochschulstiftung and NF grant 31.31018 .91 to ABC.
S12-22

ROLE OF THE TRANSGLIAL TUBULAR SYSTEM FOR THE IMPULSE CONDUCTION VELOCITY IN CRAYFISH GIANT AXON. H.M. Digicaylioglu, B. Mock and N.G. Greeff. Physiologisches Institut, Universität Zürich, CH-8057 Zürich.

Previously, the role of the transglial tubular system was shown for the squid giant axon: In $\mathrm{Na}^{+}$free (TRIS) solutions the series resistance increased in correlation with a decrease of the tubular opening density (TOD). In the crayfish giant axon, which show similar tubules, we correlate the change in TOD, measured in freeze-fracture preparations, with the conduction velocity. The control TOD was estimated to be 16 per $\mu^{2}$. After 30 and 75 min TRIS-Inkubation the TOD decreased to 10,4 and 1,5 , respectively. This reaction was reversible when these axons were

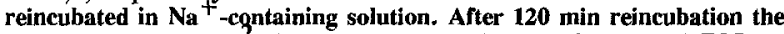
TOD was 11.8 per $\mu \mathrm{m}^{2}$. To determine the influence of decreased TOD on the impulse conduction velocity, the nerve was incubated for $30 \mathrm{~min}$ in TRIS solution followed by reincubation. Impulse propagation then recovered in two phases. During an initial fast phase with a short time constant of 1-2 min $\mathrm{Na}^{+}$diffused back into the periaxonal space and allowed impulses to occur with a low conduction velocity. In the second phase with a long time constant of $7 \mathrm{~min}$, conduction velocity recovered slowly to normal values. This time course was comparable to the recovery of TOD, as estimated from recent freeze-fracture preparations after short reincubation. These results indicate that the normal TOD is a necessary condition for the normal excitability of the axon and determines its conduction velocity. (Supported by Swiss NF grant 31-27788.89)

\section{$512-23$}

FUNCTIONAL MODULATION OF SODIUM CHANNELS BY INTERNAL CALCIUM AND PHOSPHATASE BLOCKERS.

M. Egger and N.G.Greeff. Physiologisches Institut, Universität Zürich, Winterthurerstr. 190, CH-8057 Zürich, Switzerland.

Our previous finding that whole cell sodium currents in neuroblastoma cells (N1E115) are about doubled in $100 \mathrm{nM}\left[\mathrm{Ca}^{+}\right]_{\text {, }}$ as compared to $1 \mathrm{nM}$ was further investigated. This $\mathrm{Ca}^{2+}$ meditated effect acts independently from the $\mathrm{Ca}^{2+}$ buffer system $\left(\mathrm{Ca}^{2+} / \mathrm{EGTA}\right.$ or $\mathrm{Ca}^{2+}$ /BAPTA) used. We also show that there are no changes in the steady- state inactivation and activation process. In order to study further the $\left[\mathrm{Ca}^{2+}\right]_{i}$ induced modulation of $I_{\mathrm{Na}}$ the phosphatase blockers ocadaic acid and fluoride were used. Ocadaic acid ( $30 \mathrm{nM}$ ) in the $\mathrm{PCa} 9$ and 7 (EGTA buffered) perfusion media increased $/ \mathrm{Na}$ to an equal level which is about $50 x$ higher than in PCa 9 alone. In addition, using nonstationary fluctuation analysis of $l_{\mathrm{Na}}$ we could demonstrate the single channel conductance (about $0.58 \mathrm{pA}$ ) to be the same under all conditions. Therefore, the significantly higher current densities at $\mathrm{pCa} 7$, fluoride and under ocadaic acid could be explained in terms of an increased number of activatable sodium channels. This significant increase of $l$ induced by the phosphatase inhibitors and/or high internal free $\mathrm{Ca}^{2+}$ compared with pCa 9 alone suggests a strong crosstalk between $\left[\mathrm{Ca}^{2+}\right]_{i}$ and phosphorylation processes which results in a functional modulation of the number of activatable $\mathrm{Na}^{+}$channels in the cellular membrane. (Supported by Swiss National Fund 31-27788.89)

\section{$512-24$}

CO-LOCALIZATION OF GABA - AND GLYCINE-RECEPTORS AT GABAERGIC SYNAPTIC CONTACTS IN RAT SPINAL CORD. Bohlhalter S., Mohler H., and Fritschy J.M., Institute of Pharmacology, University of Zurich, Zurich

Most spinal cord neurons respond to the inhibitory action of GABA and glycine, suggesting co-expression of $\mathrm{GABA}_{\mathrm{A}^{-}}$and glycine receptors in individual cells. While glycine-receptors are exclusively found in post-synaptic densities, the cellular localization of $\mathrm{GABA}_{\mathrm{A}^{-}}$ receptors has not yet been characterized. In this study, the distribution of GABA -receptors in the spinal cord was analyzed with an antiserum recognizing the $\alpha 1$-subunit. Double- and tripleimmunofluorescence staining were employed to identify synaptic receptors apposed to GABAergic terminals (immunoreactive for glutamic acid decarboxylase) and to assess their co-localization with glycine-receptors (visualized with an antibody to the $93 \mathrm{kDa}$ protein gephyrin). Staining for the $\mathrm{GABA}_{\mathrm{A}}$-receptor $\alpha 1$-subunit decorated the soma and dendrites of numerous neurons in laminae III-VIII and X, revealing their morphology in clear detail. By contrast, laminae II and IX contained little immunoreactivity for these $\mathrm{GABA}_{\mathrm{A}}$-receptors. Most $\mathrm{GABA}_{\mathrm{A}}$-receptor-positive cells also exhibited a prominent glycine-receptor immunoreactivity. Both types of receptors had very similar distribution patterns and were frequently co-localized in sites apposed to GABAergic boutons. These results indicate that GABA $A^{-}$ and glycine-receptors may co-exist within single post-synaptic densities, suggesting a possible synergism between GABA and glycine neurotransmission in spinal cord neurons. 
$512-25$

DIAMETER MEASUREMENT OF ISOLATED MICROVESSELS Skutella $M$ and Rüegg UT, Univ. of Lausanne, $C H$. Aim: Investigation of $\mathrm{Ca}^{2+}$-handling mechanisms involved in regulation of vascular resistance. Method: Lumen diameter measurement in mesenteric resistance arteries of the rat (diameter ca. $200 \mu \mathrm{m})$ under steady pressure $(40 \mathrm{~mm} \mathrm{Hg})$ with a video dimension analyzer after application of different drugs.

Results: Depolarization: Contractions with ma-

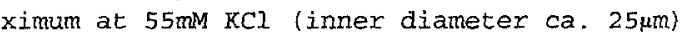
[Arg ${ }^{8}$ vasopress in and noradrenal ine: $\mathrm{EC}_{50} 2 \cdot 10^{-10}$ $M$ and $3 \cdot 10^{-7} \mathrm{M}$. Oscillations of the vessel at intermediary to high concentrations (amplitude up to $100 \mu \mathrm{m})$; completely abolished by the Ltype calcium channel antagonist diltiazem (full dilatation of the vessel).

Conclusion: Method allows conditions close to physiological function, extra- and intraluminal application and intact endothelial response. Supported by the Swiss Nat1. Science Foundation (grant $\mathrm{Nr}$. 31-36514.92)

\section{S12-26}

PRE- AND POSTNATAL EEXPRESSION OF MRNA FANCODING FOR $\alpha_{1}-, \alpha_{5}-, \beta_{2}-$ AND $\gamma_{2}$-SUBONITS OF GABA RECEATTORS IN THF RAT' CNS 'ARTIER PRENATAL EXXPOSURE TO BEHZODIAZEPINES

Ali Reza Salili, Margret Schlumpf and walter Lichtensteiger, Institute of Pharmacology, University of Zurich, CH-8057 Zurich

Prenatal benzodiazepine (BDZ) exposure changes both behavioral and neurochemical parameters of developing rats. These changes are not a consequence of remaining $B D Z$ in brain tissue, but rather indicate alterations in $B D Z$ receptor binding and function. Since the $\mathrm{BDZ}$ binding site is located on the $\mathrm{GABA}_{\mathrm{A}}$ receptor complex, it is possible that prenatal $\mathrm{BDZ}$ treatment influences the expression of GABA, receptor subunits. To evaluate effects of prenatal BDZ exposure on mRNAs expression specific for several GABA receptor subunits $\left(\alpha_{1}, \alpha_{5}, \beta_{2} \& \gamma_{2}\right)$, we treated pregnant rats with diazepam ${ }^{\prime}(1.25 \mathrm{mg} / \mathrm{kg} / \mathrm{d} ;$ s.c.) from gestational day 14 to 20. We then analyzed specific mRNA levels in offspring at four different developmentai stages (GD2O, PN8, PN15 \& adult) by in situ hybridization. Preliminary data from optical density measurements indicate a decrease in expression of mRNA in particular for $\alpha_{1}$-subunit of GABA receptors in different brain regions of prenatally diazepam-treated rats.
S12-27

CHARACTERIZATION OF NMDA RECEPTOR MEDIATED SYNAPTIC COMPONENTS OF THE THALAMIC INPUT TO CAT AUDITORY CORTEX. T. Krucker C.L. Meier, P.L. Herrling*. Sandoz Research Institute, P.O.Box, CH-3001 Bern, SWITZERLAND.

Processing of sensory information within the auditory system has been well characterized using histological and electrophysiological techniques. However, relatively little is known about the neurotransmitters involved. The present study was performed to elucidate the possible role of excitatory (EAA) and inhibitory (i.e., GABA) amino acids in neurotransmission within the primary auditory cortex (Al). The main afferent to the Al, the ipsilateral medial geniculate body (MGB), was electrically stimulated and evoked responses were recorded intracellularly in the $\mathrm{Al}$ region in halothane anesthetized cats. A seven-barrelled iontophoresis pipette glued alongside the recording electrode allowed localized application of EAAergic and GABAergic compounds onto the recorded neuron. In most Al neurons, MGB stimulation evoked a short latency, short duration EPSP followed by a long-lasting IPSP. Pattern, duration, and amplitude of synaptic potentials were highly variable and strongly dependent on stimulation intensity. Reduction of $\mathrm{GABA}_{\mathrm{A}}$ receptor-mediated inhibition by iontophoretic application of either bicuculline or SR95531 markedly enhanced MGB stimulation-evoked EPSPs. Only the late component of this enhanced EPSP was reversibly blocked by the competitive NMDA receptor antagonist AP7 at currents which selectively blocked neuronal excitation induced by iontophoretic application of NMDA. Our results demonstrate that in the cat 1) NMDA receptors in Al are activated following $M G B$ stimulation and that 2) a dominant $G A B A_{A}$ receptor mediated inhibition usually masks this NMDA receptor activation under our experimental conditions. 


\section{Molecular Genetics and Development of Higher Plants}

S13-01

AN ENDO-1,5- $\alpha$-L-ARABINANASE (EC 3.2.1.99) FROM ASPERGILLUS NIGER: PURIFICATION AND PROPERTIES.

Dunkel M.P.H. and Amadò R., Laboratory of Food

Chemistry and Food Technology, Department of Food Science, Swiss Federal Institute of Technology, zürich (Switzerland).

A highly purified and specific cell wall degrading endo-1,5- $\alpha-L$-arabinanase was isolated from an $A$. niger pectinase preparation by low and medium (FPLC) pressure column chromatographic separation methods. The isolated enzyme was most active on linear $1,5-\alpha-L$-arabinans ( 90 I.U./mg). whereas branched arabinans from sugar beets were degraded to a lesser extent $(-14 \mathrm{I} . \mathrm{U} . / \mathrm{mg}\rangle$. The enzyme was shown to be electrophoreticaliy pure after silver staining (SDS-PAGE) and its identity was confirmed through specific binding to an antiserum directed against endo-1,5- $\alpha-\mathrm{L}$-arabinanase. The major physico-chemical characteristics of the enzyme were the following: Mr 42'000, IEP $\leq 3.0$, $\mathrm{pH}$ optimum 4.8, temperature optimum $55^{\circ} \mathrm{C}, \mathrm{pH}$ stability $3.5-8.0$, temperature stability $\leq 45^{\circ} \mathrm{C}$, $\mathrm{Km}=0.205 \mathrm{mg} / \mathrm{ml}, \quad V_{\max }=17.7 \cdot 10^{-3} \mu \mathrm{mol} / \mathrm{min} . \mathrm{Zn}$ and Hg showed potent inhibitory effects.

\section{S13-02}

\section{CLONING AND EXPRESSION IN YEAST OF A HIGHER} PLANT CHORISMATE MUTASE.

Jenny Eberhard and Jürg Schmid

Institute of Plant Sciences, ETH-Zürich, 8092 Zürich.

Chorismate mutase (EC 5.4.99.5) catalyzes the first step in that branch of the shikimate pathway which leads to the aromatic amino acids phenylalanine and tyrosine. We have isolated a cDNA for this enzyme from the higher plant Arabidopsis thaliana by complementing a yeast strain (aro7) with a cDNA library from $A$. thaliana. This is the first chorismate mutase cDNA isolated from a plant. The $A$. thaliana chorismate mutase expressed in yeast revealed allosteric control by the three aromatic amino acids as previously described for plastidic chorismate mutase isozymes.

\section{S13-03}

Changes in Apical Meristem Gene Expression Patterns on the Transition to Flowering in Tomato Fleming $A$. J. and Kuhlemeier $C$., Institute of Plant Physiology, Bern University,

Our previous work has shown that transcripts for a number of genes are distributed within the vegetative apical meristem in a characteristic number of distinct patterns. To analyse how stable these patterns are, we have extended our investigation to include the transition from the vegetative to the floral state. The results of this analysis indicate that predictable changes in the spatial patterns of gene expression occur within the apical meristem on this transition. The significance of these data with respect to oreviously postulated models of floral

levelopment will be discussed.
S13-04

IMMUNOLOCALIZATION OF GLYOXYSOMAL MALATE
SYNTHASE FROM SOYBEAN COTYLEDONS (Glycine max L.

Jaunin F., Henry H., Richter H., Collet Y., Hofer R. and Widmer, F. Institut de Biologie et de Physiologie Végétales de l'Université, CH1015 Lausanne.

Malate synthase (MS; EC 4.1.3.2) is a specific enzyme of the glyoxylate cycle, which plays a key role in the initiation of gluconeogenesis in germination oilseeds. This pathway is localized in glyoxysomes, single membrane organelles which are converted into peroxisomes when lipid reserves are depleted.

The glyoxylate cycle enzymes have been the subjects of numerous conflicting reports typically addressing the problems of their synthesis (on free or bound ribosomes), targetting and suborganelar localization (membrane bound or matrix proteins).

Recent biochemical studies have shown that MS from germinating soybean cotyledons is an interconvertible enzyme (membrane bound, aggregated or soluble forms) which is significantly affected by its ionic environment (Henry et al., 1992, Plant Science 82:21-27).

Microscopy studies have now been carried out using immunofluorescence staining or immunogold-silver staining for light microscopy, and inmunogold labelling for electron microscopy. All results consistently characterize MS as a glyoxysomal matrix enzyme.

\section{S13-05}

INFLUENCE OF ENVIRONMENTAL CONDITIONS ON THE CATABOLISM OF RUBISCO IN INTACT CELLS

A. Fischer, M, Hildbrand, P. Stieger and U. Feller, Institute of Plant Physiology, Altenbergrain 21, $\mathrm{CH}-3013$ Bern

An attachment of Rubisco to chloroplast membranes under $\mathrm{Cu}^{++}$-stress has been described for wheat (Mehta et al., J. Biol. Chem. 267, 2810-2816). The displacement to the membranes has been discussed as a possible step in the degradation of this predominant stromal protein, e.g. during leaf senescence. In our recent experiments it became evident that such interactions are not restricted to Rubisco. Several other stromal, and even a peroxisomal enzyme (glycolate oxidase), were also detected in the membrane fraction after 6 to 30 hours of oxidative stress induced in wheat seedlings by a treatment

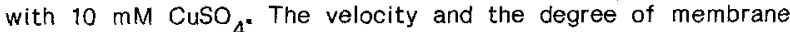
attachment varied for different enzymes. Based on these results it was interesting to check the solubility of Rubisco and its previously described $45 \mathrm{kD}$ fragment which accumulates during the incubation of bean leaf discs under oxygen deficiency (Hildbrand and Feller, Experientia 47, A67). Neither Rubisco nor the breakdown product were found to accumulate in the membrane fraction. Thus, at present, the steps involved in Rubisco catabolism cannot be generalized. Different mechanisms depending on the metabolic situation and on environmental conditions should be considered.

\section{S13-06}

Overexpression of the four parsley phenylalanine ammonia-lyase (PAL) isoenzymes in E. coli. Characterization of the enzymes and kinetic analysis of the inhibition by 2-aminoindan-2-phosphonic acid.

Christoph Appert, Jürg Schmid, Jerzy Zoń* and Nikolaus Amrhein

Institute of Plant Sciences, ETH-Zürich, 8092 Zürich.

*Technical University Wroclaw, 50-370 Wroclaw, Poland.

All four phenylalanine ammonia-lyase (EC 4.3.1.5) isoenzymes of parsley (Petroselinum crispum Nym.) were expressed as glutathione S-transferase fusion proteins in E. coli. After affinity purification, the glutathione Stransferase moieties were cleaved off with factor $\mathrm{Xa}$ and the phenylalanine ammonia-lyases were characterized. The proteins form enzymatically active tetramers, even as fusion proteins. The four isoenzymes have comparable $\mathrm{K}_{\mathrm{m}}$ values for $\mathrm{L}$-phenylalanine ( $15 \mu \mathrm{M}$ to $\left.24.5 \mu \mathrm{M}\right)$, similar temperature $\left(58^{\circ} \mathrm{C}\right)$ and $\mathrm{pH}$ optima $(8.5)$. The aminooxy-and phosphonic-analogues of $\mathrm{L}$-phenylalanine are competitive inhibitors of the enzymes. 2-Aminoindan2-phosphonic acid (J. Zon \& N. Amrhein, Liebigs Ann. Chem. 1992,625) was found to be a potent slow-binding inhibitor of these and other phenylalanine ammonnia-lyases, both in vivo and in vitro. 
S13-07

SALICYLIC ACID AND PATHOGENESIS-RELATED PROTEINS IN ROOTS OF INFECTED CUCUMBER PLANTS

I. Sticher, P. MeuwIy and J.-P. Métraux

Institut de Biologie végétale, Université de

Fribourg, 3, rue Albert Gockel, 1700 Fribourg.

The attack of plants by pathogens which provoke local necrosis may lead to subsequent resistance to pathogens both locally and systemically in distant tissues. The resistance is correlated with the appearance of pathogenesis-related (PR) proteins andthe increase of salicylic acid (SA) at the site of infection. A role for $S A$ as a signal which travels to the other parts of the plant to induce systemic resistance has been oroposed. So far, systemic reactions have been studied in leaves. We examined the response of the roots to the infection of cotyledons of cucumber plants with Pseudomonas lachrymans. Free and conjugated $S A$ increased in the roots after infection of the cotyledons. The appearance of $P R$ proteins including chitinase was also examined.

S13-09

BIOSYNTHESIS OF SALICYIIC ACID IN INFECTED CUCUMBER PLANTS

Meuwly P., Mölders $W$. and Métraux J.-P.

Institut de Biologie végétale, Université de Fribourg, 1700 Fribourg, Switzerland

Salicylic acid (SA) was proposed as a putative signalling molecule for the induction of systemic acquired resistance (SAR) in infected plants. We studied its biosynthesis as follows.

Cotyledons of cucumber plants were injected with a solution containing PSeudomonas lachrymans and fed with radioactive SA precursors by injection of $14 \mathrm{C}$-benzoic acid (14C-BA) or $14 \mathrm{C}-$ phenylalanine $(14 \mathrm{C}-\mathrm{Phe})$. Alternatively, leaf 1 of cucumber plants were inoculated with tobacco necrosis virus (TNV) and leaf disks were then vacuum-infiltrated using $14 \mathrm{C}-\mathrm{BA}$ or $14 \mathrm{C}$-Phe. Free and bound $14 \mathrm{C}$-phenolics were quantified by HPLC.

Incorporation of ${ }^{14} \mathrm{C}$ into $\mathrm{SA}$ was found in the two plant-pathogen systems. 14C-BA gave mostly gave also some $14 \mathrm{C}-\mathrm{SA}$, in addition to ${ }^{14} \mathrm{C}$-labelied lignin precursors like ferulic and p-coumaric acids. The biosynthetic pathway of SA from Phe through cinnamic acid and BA will be discussed.

\section{S13-08}

TRANSPORT OF SALICYLIC ACID AND ITS PRECURSORS IN CUCUMBER PLANTS

Mỏlders W., Meuwly P., and Métraux J.-P.

Institut de Biologie végétale, Université de

Fribourg, 1700 Fribourg, Switzerland

After infection with a necrotizing pathogen, systemic acquired resistance (SAR) is often observed in plants. In cucumber, salicylic acid (SA) increases in the lower infected leaf as well as in the upper uninfected leaves. SA was also found in the phloem sap of infected cucumber plants and was proposed as a signalling molecule for the induction of SAR. We intend to clarify whether SA is translocated from the site of infection to the upper leaf, or wether it is made in the phloem tissue upon the action of a primary systemic signal.

One cotyledon of cucumber plants was first infected with pseudomonas lachrymans and then fed with ${ }^{14} \mathrm{C}-S A$, ${ }^{14} \mathrm{C}$-benzoic acid (BA) or ${ }^{14} \mathrm{C}$-phenylalanine. After different times, cotyledons and first leaves were collected and the radioactive phenolics were analyzed by HPLC. In some cases, ${ }_{14} \mathrm{C}-\mathrm{SA}$ was found in the first leaf. In addition, two unknown radioactive compounds were detected in leaf 1 after treatment with ${ }^{14} \mathrm{C}-\mathrm{SA}$ and ${ }^{14} \mathrm{C}-\mathrm{BA}$ respectively. We will discuss signalling processes for the induction of SAR in cucumber.
S13-10

COMPARATIVE STUDY OF TWO VARIETIES OF POTATO (SOLANUM TUBEROSUM) SUBJECTED TO DROUGHT STRESS.

Bérouba GUISSE and Reto J. STRASSER

Laboratory of Bioenergetics, University of Geneva CH-1254, Jussy, Geneva

Chlorophyll a fluorescence has been used to compare the drought resistance of two varieties of Solanum tuberosum (AVR/DC-1287-19 AND SIRTEMA). Fast fluorescence rise kinetics were measured during the first second of illumination with a time resolution of $10 \mu \mathrm{s}$ and 12 bits in fluorescence intensity. The rise kinetics shows the typical steps called Fo - J - I - P. With this values different indexes have been defind with the goal to compare the behaviour of these two varieties of potato. Plant were subjected to drought stress. Some of the defined indexes were markedly different for the two varieties during the first period of drought stress. Rewatering the plants showed that the AVR/DC-1287-19 variety recovered to its normal behaviour whereas SIRTEMA variety showed only a partial recovery. We suggest that fluorescence techniques can be used to evaluate the drought stress resistance in plants of agricultural interest.

\section{S13-11}

EXPRESSION OF SEVERAL MODIFIED GRAPEVINE NEPOVIRUSES (GRAPEVINE FANLEAF VIRUS AND ARABIS MOSAIC VIRUS) COAT PROTEIN GENES IN TRANSGENIC TOBACCO PLANTS.

Marc-Martin S. ${ }^{1}$, Ramel M.-E. ${ }^{2}$, Gugerli P. ${ }^{2}$, Stutz E. ${ }^{1}$ and Spielmann A. ${ }^{1}$

1 Université de Neuchâtel, Laboratoire de biochimie végétale, Chantemerle $18, \mathrm{CH}-2000$ Neuchâtel; '2Station féférale de recherches agronomiques, Changins, Département de virologie, $\mathrm{CH}-1260$ Nyon

The most important soil-borne grapevine viruses are transmitted from the roots of infected vines to the neighbouring healthy ones by nematodes (nepoviruses). Three such viruses are known to occur in Swiss vineyards: grapevine fanleaf virus (GFLV), arabis mosaic virus (ARMV) and raspberry ringspot virus (RRSV). The nepoviruses cause quick destruction of young plants or a gradual decline over several years (infectious degeneration). GFLV is the most widespread virus in grapevines and is the nepovirus of greatest economic importance.

One modern approach of creating virus resistant grapevine plants is the introduction of resistance genes into existing grapevine varieties by genetic engineering. The goal of this work is to use the coat protein (CP) mediated strategy to induce resistance to nepovirus in Vitis $s p p$. As a first step, several chimeric nepovirus $C P$ genes were constructed by addition of various promoter regions upstream of the GFLV and ArMV CP regions.

Their ability of conferring resistance to nepovirus infection in transgenic Nicotiana benthamiana and $N$. tabacum plants will be presented. The best constructions will be used to transform several grapevine varieties in order to create nepovirus resistant grapevine plants.

\section{\$13-12}

Characterisation of a tyrosinase from Amanita muscaria involved in betalain biosynthesis

Lukas Müller, Ursula Hinz and Jean-Pierre Zrÿd

Laboratoire de phytogénétique cellulaire

Université de Lausanne

1015 Dorigny

The betalains are a class of natural pigments found only in plants of the order Caryophyllales and in some fungi. The first step in betalain biosynthesis is the conversion of tyrosine to DOPA. Subsequently, DOPA is transformed to betalamic acid, the betalain chromophore, through the action of DOPA-4,5-dioxygenase.

We have purified and characterised a copper-containing enzyme of $\sim 38 \mathrm{kDa}$ from Amanita muscaria pileus that catalyses the hydroxylation of tyrosine to DOPA. Considering that the enzymatic activity was restricted to the coloured parts of the mushroom, we postulate that it is involved in betalain biosynthesis. The enzyme featured a broad substrate specificity, and also oxidised the diphenols to their corresponding ortho-quinones, a reaction typical for tyrosinases. The implications of our findings on betalain biosynthesis are discussed. 
HEAT PROTECTION BY LOW LIGHT: A TYPICAI CHARACTERISTIC OF LAND PLANT

Alaka Srivastava and Reto J. Strasser Lab of Bioenergetics, University of Geneva CH-1254, Jussy, Geneva

Chlorophyll a fluorescence was measured under steady state conditions of pea and tomato leaves adapted to low light intensity $\left(30 \mathrm{Wm}^{-2} \mathrm{~s}^{-1}\right)$ at different temperatures (Havaux and strasser, $z$. Naturforsch. 45C, 1133-1141, 1990). When leaves were exposed for a short period (10 $\mathrm{min}$ ) to heat ( 25 to $45^{\circ} \mathrm{C}$ ) in darkness, the level of variable fluorescence decreased. When the heat stress was imposed in presence of low light, the variable fluorescence was much less affected and virtually no effect of heat treatment was observed until $37^{\circ} \mathrm{C}$. This protecting mechanism by low light was absent in algae and mostly absent in submerged water plants but fully present in free floating plants on the water surface. We conclude that a mechanism has been developed for higher land plants during evolution which protects the plants against heat damage on warmer days.

\section{S13-14}

THE EVOLOTION OF BETALAIN BIOSYNTHESIS IN PLANTS AND FUNGI

J. Fivaz, M. Zaiko, U. Hinz \& J.P. Zryd, Uni Lausanne, Laboratoire de Ehytogénétique cellulaire, Bâtiment de Biologie, 1015 Dorigny

Betalains are a group of yellow or violet pigments with a very limited taxonomic distribution. They are found only in plants of the order Caryophyllales, e.g. Portulaca grandiflora, and in a few fungal species, e.g. Amanita muscaria.

We analysed the similarity of a key enzyme of the pathway, DOPA-4,5-dioxygenase, in plants and fungi both at the protein and the DNA level. Antibodies against purified DOPA-4,5-dioxygenase from the funqus $A$. muscaria crossreacted with protein from $P$. grandiflora petals and two cDNA clones were obtained from this plant. Their similarity with fungal sequences and with other dioxygenases is discussed.
Kinetics of prollue hydroxylation, Intracellular transport and C-terminal processing of the tobacco vacuolar chittaase.

Ernst Freydl, Thomas Boller and Jean-Marc Neuhaus

Botanisches Institut, Abt. Pflanzenphysiologie, Hebelstrasse 1, CH-4056 Basel

The vacuolar chitinase A of tobacco (Nicotiana tabacum) is synthetized as a preproprotein with an N-terminal signal peptide which causes its synthesis in the ER and a C-terminal extension, which has been identified as the vacuolar targeting peptide (VTP) (1) and which is cleaved off from the mature chitinase. It has recently been shown that mature chitinase A contains several hydroxyprolines within the short peptide spacer that links the N-terminal cysteine-rich chitin-binding domain to the catalytic domain (2).

We received specific antibodies against mature chitinase (a kind gift from Dr. F.Meins, FMI) and raised antibodies against a synthetic VTP. We performed pulse-chase experiments and cell fractionation with 1) stably transformed tobacco plants expressing chitinase $A$ or mutants lacking either the chitin-binding domain and spacer (Chi $\Delta H)$ or the vacuolar targeting peptide (Chit $\Delta$ VTP) and 2) transient expression of the same constructs in Nicotiana plumbaginifolia protoplasts. In both systems, proline hydroxylation in chitinase $A$ was detectable after $30 \mathrm{~min}$. and complete after 90-120 min. The chitinase intermediate forms were detected in the microsomat and soluble fractions for up to $90-120$ $\mathrm{min}$. The mabure chirinase continuously accumulated only in the soluble fraction. In both

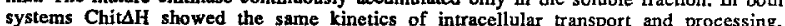
whereas the transport of ChitSVTP seemed more rapid.These tesulss indicate that in vivo chitinase $A$ is synthetized as a proprotein that is then made This second intermediste form is vacuole. C-terminal processing occurs late in this pathway or in the vacuole.

1. Neuhaus, J.-M., Sticher, L., Melns, F. and Boller, T. (1991). Proc. Natl. 1. Neuhaus, J.-M., Sticher, L.,

2. Sticher, L., Hofsteenge, J., Neubaus, J.-M., Boller, T. and Meins, F., 2. Sticher, L., Hofsteenge, J., Neubaus,
Jr. (1993). Plant Physiology 101, 1239-1247.

\section{S13-17} Structural and functional analysis of chitinase
isoenzymes in Fusarium solani-infected bean roots

Uwe Mohr, Jürg Lange, Regina Vögeli-Lange, Thomas Boller and Andres Wiemken Botanisches Institut, Hebelstr. 1, CH-4056 Basel

Infection of bean roots with the soil-borne phytopathogenic fungus Fusarium solani f.sp. phaseoli leads to a rapid increase of chitinase activity ( $\sim$ five-fold). Part of this increase in enzyme activity is due to transcriptional activation of the basic class I chitinase isoenzymes. Semi-native polyacrylamide gels stained for chitinase activity using the substrate glycol-chitin revealed the appearence of three additional chitinase isoenzymes that are absent from uninfected control roots. Conventional protein purification techniques showed that Fusarium solani infected bean roots contain two basic and two acidic chitinase isoenzymes. Their physiochemical properties and possible biological function will be discussed.
S13-15

PURIfication and CHARACTERISATION OF THE PROTEASOME FROM THE THE MOSS Physcomitrella patens

Richard C., Girod P.-A. \& Zryd J.-P.

Laboratoire de Phytogénétique Cellulaire, Université de Lausanne, 1015 Lausanne

Proteasomes are multicatalytic protease complexes that function as a major nonlysosomal proteolytic system. They exhibit multiple endopeptidase activities that promote the intracellular turnover of abnormal polypeptides and short-lived regulatory proteins. Moss proteasome has been purified from protonemata by successive chromatography on MacroPrep Q, Bio-Gel A-1.5, Bio-Gel A-5 and HA The molecular mass was estimated to be $1,300 \mathrm{kD}$ by gel filtration. Gel electrophoresis of proteasome under nondenaturing condition gave a single band and two-dimensional gel electrophoresis identified the moss proteasome to be composed of 14 different subunits with molecular weight in the range 22-35 kD. Electron microscopy in aqueous solution showed that it is a cylindrical particle composed of four stacked rings with a diameter of $9 \mathrm{~nm}$ and a height of $14 \mathrm{~nm}$. End-on projection established a 7-fold symmetry of the outer disks. Substrate specificity of proteasome indicates that it contains endopeptidase activity against substrates bearing hydrophobic, basic, acidic and glycine residues immediately preceding the cleavage site. Antibodies raised against moss proteasome reacted with proteasomes of other eukaryotes, including human. 


\section{Cytokines and Cytokine Receptors}

S14-01

\section{AUTOIMMUNE SIALADENITIS: DETECTION OF ACTIVATED CYTOTOXIC CELLS AND TNF- $\alpha$ EXPRESSING CELLS IN SITU} Hunger R.E., Hess M.W., Laissue J.A., Mueller C. Institute of Pathology, University of Bern

The NOD (non obese diabetic) mouse, a widely used animal model for insulin dependent diabetes mellitus, shows in addition to mononuclear cell infiltration of the islets of Langerhans, massive cellular infiltrates of the submandibular and lacrimal glands with considerable tissue damage. Several lines of evidence suggest that both insulitis and sialadenitis represent autoimmune disorders.

To investigate a possible role of tumor necrosis factor- $\alpha$ (TNF- $\alpha$ ) and activated cytotoxic cells (NK cells, $T$ cells) in the inflammatory process, tissue sections of NOD mice were hybridized with radiolabeled RNA probes specific for the detection of mRNA for TNF- $\alpha$ and the serine proteases granzyme $A$ and $B$ (GrA and $\mathrm{GrB}$ ) and perforin which are expressed in activated cytotoxic cells. TNF- $\alpha$ expressing cells were mainly located in infiltrates and are absent in non affected glands, whereas GrA, GrB and perforin expressing cells were distributed over the whole section with highest densities in the zones adjacent to parenchyma. The finding that activated cytotoxic cells are present in early stage of disease development indicates that cell mediated cytotoxicity may play a crucial role in initial tissue destruction. The role of TNF-a in autoimmune diseases is not known yet. The appearance, however, of cells expressing this gene in the infiltrate indicates a possible role of this cytokine in the progression of the inflammatory reaction, e.g. by increasing the lymphocyte traffic to this organ.

\section{S14-02}

CHARACTERIZATION OF THE PROMOTER REGION OF THE RAT GENE CODING FOR THE INDUCIBLE NITRIC OXID SYNTHASE

Wolfgang Eberhardt, Dieter Kunz and Josef Pfeilschifter, Dept. Pharmacology, Biocenter, University of Basel, $\mathrm{CH}-4056$ Basel

We previously have shown that activation of rat glomerular mesangial cells by exposure to the proinflammatory cytokines such as interleukin $1 B$ (IL-1B)or CAMPelevating agents can induce a macrophage-type of nitric oxide synthase (NOS). We further demonstrated that the induction of iNOS was at the transcriptional level. In order to understand the underlying mechanisms we characterized the promoter region of the rat NOS gene. A genomic rat library was screened using a CDNA-fragment coding for the 5 -end of the INOS CDNA (nucleotides 1- 817). A $1,8 \mathrm{~kb}$ Hinc-1l fragment containing the 5 - flanking region of the iNOS gene was characterized by DNA-sequencing. The transcriptional start site was determined by primer extension. Sequencing analysis revealed a multitude of possible cis-actíng elements homologues to consensus sequences for the binding of different transcription factors including IFN $\gamma$ response element (IRE), nuclear factor-kB (NF$\kappa B$ ), tumor necrosis factor response element (TNF-RE), $\gamma$-activated sites (GAS) and one $\mathrm{X}$-box. NF-kB, a transcription factor involved in signaling and immediate early gene activation during inflammatory processes was induced by treatment of mesangial cells with $2 \mathrm{nM}$ of $\mathrm{IL}-1 \beta$. This was shown by the appearence in nuclear extracts of the DNA binding activity of NF-KB using electrophoretic mobility shift assays (EMSAS) with a 32 p-labeled $K B$ DNA probe. Pyrrolidinedithiocarbamate (PDTC), an inhibitor of NF-KB, suppressed the expression of iNOS mRNA in mesangial cells without affecting the Dibutyryl-cAMP-triggered increases in NOS mRNA levels. This data suggest that the $\mathrm{L}-1 \beta$ signalling pathway responsible for NOS expression requires NF- $\mathrm{KB}$ activation and is definetly different from the signalling cascade directed by cAMP.

\section{S14-03}

EXPRESSION OF INTERLEUKIN-6 (IL-6) AND IL-6 RECEPTOR (IL-6R) MRNAS IN RAT BRAIN DURING POSTNATAL DEVELOPMENT

R.A.Gadient and U.Otten, Dept.Physiology, University of Basel, Vesalianum, Vesalgasse 1 , $\mathrm{CH}-4051$ Basel

Recent findings indicate that the multifunctional cytokine IL-6, which plays a key role in immune and inflammatory responses, also exerts specific effects in the central nervous system (CNS). For example, IL-6 promotes neuronal survival, induces differentiation and modulates neurotrophin production.

Using the very sensitive technique of reverse transcription combined with polymerase chain reaction the developmental profile of $I L-6$ and its receptor mRNAs was analyzed in various rat brain regions. Our results indicate that both genes are expressed in a region-specific manner and are developmentally regulated. These findings support the hypothesis that IL-6 is involved in differentiation and maintenance of neuronal subpopulation in the CNS.
S14-04

T CELL UNRESPONSIVENESS INDUCED BY TRANSLATIONAL REGULATION OF IL-2 mRNA. J.A. Garcia-Sanz and D. Lenig, Basel Institute for Immunology, $\mathrm{CH} 4005$ Basel, Switzerland

Interleukin 2 (IL-2) expression is strictly dependent on a signal transmitted by the $T$ cell receptor upon antigen stimulation. This signal can be mimicked in vitro with phorbol esters and calcium ionophores. We have found that stimulation with $\mathrm{Ca}$-ionophore alone induces expression of IL-2 mRNA, but does not induce secretion of IL-2.

In polysome gradient fractionation of cells stimulated with phorbol esters and ionophore, the fractions containing IL-2 MRNA are found at the bottom of the gradient, bound to polysomes. However, in ionophorestimulated cells the fractions containing IL-2 mRNA are clearly shifted to the top of the gradient, and therefore are not translated. This effect is specific for IL-2, as other mRNA's like $\beta 2$-microglobulin are not regulated. This data suggests that IL-2 gene is translationally regulated. In addition, in vitro experiments have shown that the lack of translation of IL-2 mRNA in ionophore-stimulated cells is due, most likely, to the presence of a translational regulator that specifically inhibits translation of IL-2 mRNA. Assuming the presence of a translational regulator that specifically represses IL-2 mRNA translation, we can predict that, if the repressor is in excess, even upon a second stimulus that is by itself able to induce secretion of IL-2, there will be no translation of IL-2 mRNA. The results of such an experiment confirmed our prediction. Polysome gradients showed that after ionophore stimulation, IL-2 mRNA gets "stacked" with one ribosome, and no further ribosome loading takes place.

S14-05

TNF $\alpha$ REGULATES THE LPS-INDUCED NITRIC OXIDE PRODUCTION IN CULTURED RAT HEPATOCYTES.

B. Saad, R. Péclard, M. Christoffel, H.P. Schawalder, P. Maier and $B$. Ryffel. Institute of Toxicology, ETH and University of Zürich, $\mathrm{CH}-8603$ Schwerzenbach, Switzerland

Regulation of nitric oxide (NO) production in lipopolysaccharide (LPS) induced liver injury is thought to occur in response to Kupfer cell-derived cytokines. In this study we used rat hepatocytes cultured under improved culture conditions to investigate the direct effects of LPS on the production of NO. Exposure of hepatocytes to LPS $(10 \mu \mathrm{g} / \mathrm{ml})$ for 48 hours increased the NO production by $28 f \circ$ d. LPS induced the synthesis and release of TNF $\alpha$. Neutralizing anti-TNF $\alpha$ antibodies reduced the LPS-induced NO production by $60 \%$. Preincubation with $\mathrm{GdCl}_{3}(10 \mu \mathrm{g} / \mathrm{ml})$ did not change the response of cultured hepatocytes to LPS. These findings indicate that LPS can induce directly, Kupffer cells independent, NO production in cultured rat hepatocytes via hepatocyte-derived $T N F \alpha$

\section{S14-06}

IMMUNOLOGICAL MARKERS OF CHILDHOOD FEVERS IN AN AREA OF HIGHLY ENDEMIC MALARIA N. Hurt ${ }^{2}$, M. Thein ${ }^{3}$, T. Smith ${ }^{1,2}$, G. Bordmann ${ }^{2}, N$. Drees $^{3}$, H. Gallati $i^{3}$, M. Tanner, ${ }^{1,2}$ N. Weiss ${ }^{2}$ ${ }^{1}$ Ifakara Centre, PO Box 53, TZ-Ifakara, ${ }^{2}$ Swiss Tropical Institute, $4002 \mathrm{Basel},{ }^{3} \mathrm{Hoffmann-La}$ Roche LTD, 4002 Basel

If malaria is highly endemic, every febrile child is a presumptive malaria case, and there is no differential diagnosis based on clinical, immunological or parasitological grounds. We evaluated the diagnostic usefulness of interleukin-2 receptor (IL-2R), tumor necrosis factor-receptors 55 (TNF-R55) and 75 (TNF-R75). Sera came from Tanzanian pediatric fever patients and controls, all enrolled in the Kilombero Malaria project. We found that all 3 receptors marked pediatric fever episodes, whereas stability of observed levels was highest for TNF-R75 and lowest for TNF-R55. TNF-R55 levels reflected severity of the fever attack. Regardless of the presence of a Eebrile illness, TNF-R75 levels were strongly associated with parasite density. Immunological markers can be applied in rapid pre- and post-intervention morbidity assessments, validation of health interviews and monitoring of convalescence. 
HUMAN PERIPHERAL BLOOD EOSINOPHLS CONTAIN AND RELEASE PREFORMED INTERLEUKIN-8

S. Yousefi, F. Erard, K. Blaser and H.-U. Simon.

Swiss Institute of Allergy and Asthma Research, Davos.

We have recently shown that human eosinophils produce mRNA for interleukin (IL)-8 when stimulated with calcium ionophore (Eur. J. Immunol. 23 (1993), 956). We now present evidence that human eosinophils contain preformed $\mathrm{IL-8}$ protein although they do not express mRNA for IL-8 as measured by RT-PCR. IL-8 protein expression was determined using specific anti human IL -8 monoclonal antibodies by Westem blotting, FACS analysis and immunohistochemistry. Moreover, preformed IL-8 was released into supernatant by $99 \%$ pure eosinophils after priming with GM-CSF or IL-5 and subsequent 25-min stimulation with PAF, RANTES, ionomycin or PMA, however not with IL-1 or IL-2, as measured by ELISA. This observation implies that activation of protein kinase C and/or intracellular calcium mobilization are necessary events for IL-8 release in human eosinophils. The determined amounts of preformed IL-8 protein was higher in patients with asthma compared to normal individuals suggesting a role for eosinophil derived IL-8 in asthma. Since the eosinophil is the predominant cell in the asthmatic airways. we determined IL-8 concentrations in bronchoalveolar lavage (BAL) fluids from normal individuals and asthmatic patients. Indeed, IL-8 concentrations in BALs from both groups were similar to those seen in the supernatants released by activated eosinophils. The role for IL-8 in asthma remains to be determined.

\section{S14-08}

TUMOR NECROSIS FACTOR- $\alpha$ AND INTERFERON- $\gamma$ ARE SELECTIVELY CYTOSTATIC FOR HUMAN BREAST CANCER CELLS.Heinz Mueller, Nathalie Flury, Sibylle Scheidegger and Urs Eppenberger. Department of Research and Department of Gynecology, University of Basel, CH-4031 Basel

TNF- $\alpha$ had dose-dependent antiproliferative activity on hormone-dependent human breast cancer cells that represent an early stage of the disease, whereas hormone-independent cells representing a late stage were not affected. However, in the presence of $1000 \mathrm{U} / \mathrm{ml}$ interferon $\gamma$ (INF), high concentrations of TNF- $\alpha$ (1nM) were able to inhibit the growth of hormone-independent cells. Using specific monoclonal antibodies in flow cytometry, no significant changes of either the p75 or the p55 TNF receptors were observed upon INF treatment of hormone-independent cells. This indicates that the increased responsiveness of these cells for TNF- $\alpha$ is not due to a change in available TNF receptors. The affinity of the receptors for TNF- $\alpha$ are one order of magnitude different for the two cell types. INF treatment shifted the $\mathrm{EC}_{50}$ of hormone-independent cells towards the $\mathrm{EC}_{50}$ of hormone-dependent cells. Thus, the basis for the increased TNF-sensitivity of hormone-independent cells in the presence of INF might be the interconversion of TNF receptors from low- to high-affinity.

\section{S14-09}

\section{TUMOR CELLS REGULATE NO PRODUCTION INDUCED BY CYTOKINES IN BRAIN-DERIVED ENDOTHELIAL CELLS}

Murata, J.I., Janzer R.C. and Juillerat-Jeanneret, L.

Division of Neuropathology, Bugnon 27, 1011 Lausanne

The adhesion of tumor cells to endothelium is the first step in the processus of metastasis, and is frequently dependent of cytokinemediated activation of endothelial cells. Cytokines are also involved in nitric oxide (NO) production by various cells. These experiments were designed to evaluate NO production during tumor cell adhesion to endothelium. Rat brain-derived endothelial and rat colon carcinoma cell lines were used during these experiments. NO was evaluated with the Griess reagent. Activation of endothelial cells with cytokines (TNF- $\alpha$ and IFN- 8 ), only slightly increased $(10-20 \%)$ the adhesion of tumors cells to endothelium. Activation of endothelial cells with TNF- $\alpha$ and IFN- $\delta$ induced a 4-8 fold increase of NO production. However, addition of tumor cells to the endothelial monolayer, either directly or in a semi-permeable membrane, decreased the cytokine-induced NO production. These results indicate that NO production is modulated during the processus of adhesion of tumor cells to endothelium.
EFFECTS OF HDL AND HDL ASSOCIATED CANTHAXANTHIN IN VITRO: TISSUE SPECIFIC RESPONSE ON HDL AND INDUCTION OF CRYSTAL FORMATION BY CANTHAXANTHIN.

A. Bruinink $\left.{ }^{*}\right), W . \mathrm{Cohn}^{+}$), C Sidler $\left.{ }^{*}\right), \mathrm{H} . \mathrm{Grun}^{+}$), $\mathrm{HW}$ Wiser ${ }^{+}$)

") Institute of Toxicology, ETH \& Uni Zürich. $\mathrm{CH}-8603$, Schwerzenbach and

+)Dept. of Vitamin Research, F. Hoffmann-La Roche Lid, CH-4002 Basel

To study the effect of canthaxanthin in vitro, the hydrophobic compound was introduced in vivo into chick high density lipoproteins (HDL) by canthaxanthin feeding. The effects of $\mathrm{HDL}$ and $\mathrm{HDL}$ associated canthaxanthin on two types of cultures have been tested: flat sedimented cell cultures of embryonic chick neuronal retina, retinal pigment epithelium (RPE), brain and meninges, and in reaggregate cell cultures of the neuronal retina. At high canthaxanthin concentrations the formation of colored, birefringent entities were induced in the neuronal retina in vitro. In line with human data, parameters of cellular function and cell differentiation remained unaffected by canthaxanthin at these concentrations

By contrast, HDL was found to induce various cell type dependent effects. Proliferation of meninges cells was decreased at low HDL concentrations as concluded from.light microscopic examination and indicated by protein content, and lysosomal and mitochondrial activity of the cultures (IC50: 15-30 mg HDL apoprotein/L medium). These parameters, however, were increased in brain cell cultures, while they remained unaffected in cell cultures of neuronal retina and RPE. Concentrations above $0.3 \mathrm{~g}$. HDL apoprotein/L caused a reduction of glial cell differentiation.

\section{S14-11}

TETRAHYDROBIOPTERIN $\left(\mathrm{BH}_{4}\right)$ LIMITS NITRIC OXIDE (NO) GENERATION IN CYTOKINE STIMULATED MESANGIAL CELLS Heiko Mühl and Josef Pfeilschifter, Dept. Pharmacology, Biocenter, University of Basel, $\mathrm{CH}-4056$ Basel

NO synthase (NOS) is induced in rat mesangial cells by inflammatory cytokines such as interleukin $1 \beta(\mathrm{IL}-1 \beta)$ and requires $\mathrm{BH}_{4}$ as a cofactor. 2,4-Diamino-6-hydroxypyrimidine (DAHP), a selective inhibitor of GTP cyclohydrolase $I$, the rate-limiting enzyme for $\mathrm{BH}_{4}$ synthesis, potently suppressed IL-1 $1 \beta$ induced NOS activity, measured as nitrite production. Inhibition of NO synthesis by DAHP was reversed by sepiapterin, which provides $\mathrm{BH}_{4}$ via a salvage pathway. Sepiapterin dose-dependently augmented IL-1 $\beta$-stimulated $\mathrm{NO}$ synthesis, indicating that availability of $\mathrm{BH}_{4}$ limits the production of $\mathrm{NO}$ in cytokine-stimulated mesangial cells. $\mathrm{N}$-acetylserotonin, an inhibitor of the $\mathrm{BH}_{4}$ synthetic enzyme sepiapterin reductase, completely abolished IL- $1 \beta$-induced NO formation whereas methotrexate, which inhibits the pterin salvage pathway, displayed only a moderate inhibitory effect, thus suggesting that mesangial cells predominantly synthesize $\mathrm{BH}_{4}$ for GTP. In conclusion, these data demonstrate that $\mathrm{BH}_{4}$ synthesis is an absolute requirement for cytokine induction of NOS in mesangial cells. Inhibition of $\mathrm{BH}_{4}$ synthesis may provide new therapeutic approaches to the treatment of pathological conditions mediated by NO.

S14-12

PLATELET DERIVED GROWTH FACTOR AND BASIC FIBROBLAST GROWTH FACTOR DIFFERENTIALLY MODULATE INTERLEUKIN $1 \beta$ - INDUCTION OF THE NITRIC OXIDE SYNTHASE GENE IN RAT RENAL MESANGIAL CELLS Dieter Kunz, Gaby Walker, Wolfgang Eberhardt and Josef Pfeilschifter, Dept. Pharmacology, Biocenter, University of Basel, $\mathrm{CH}-4056$ Basel Recently we have shown that exposure of rat glomerular mesangial cells to proinflammatory cytokines such as Interleukin $\uparrow \beta(\mathrm{L}-1 \beta)$ can induce a macrophage-type of nitric oxide synthase (iNOS). Northern-blot analyses of iNOS mRNA-levels and nuclear run-on experiments of control and $\mathrm{HL}-1 \beta$ stimulated mesangial cells revealed that the induction of iNOS was at the transcriptional level. Here we demonstrate that platelet-derived growth factor $\mathrm{BB}$ (PDGF-BB) can suppress the $\mathbb{I L}-1 \beta$ dependent induction of the iNOS gene. Northern-blot analyses of cellular RNA isolated from mesangial cells that were coincubated with IL-1B (2nM) and PDGF-BB $(10 \mathrm{ng} / \mathrm{ml}, 100 \mathrm{ng} / \mathrm{ml}$ and $300 \mathrm{ng} / \mathrm{ml}$ ) revealed a dosedependent suppression of iNOS mRNA-levels. Using run-on experiments with nuclei from mesangial cells after stimulation with IL-1 $\beta$ (2nM) and PDGF-BB (100 $\mathrm{ng} / \mathrm{ml}$ ) we demonstrate that the inhibition occurs at the transcriptional level. Basic fibroblast growth factor (bFGF), on the other hand, potentiates the IL-1 13 dependent stimulation of iNOS. Coincubation of mesangial cells with IL-1B (2nM) and bFGF (3ng/ml, 10ng/ml, 30ng/ml, 100ng/ml) dose-dependently superinduces iNOS mRNA levels as shown by Northern-blot analyses of total cellular RNA form control and stimulated cells. Run-on experiments with nuclei from mesangial cells stimulated with $(L-1 \beta(2 n M)$ and bFGF (100ng/ml) revealed an enhanced transcriptional activity of the iNOS gene. Thus, PDGF-BB and bFGF differentally modulate the IL-1 13 dependent expression of the INOS gene and are therefore an excellent model system to study the crosstalk between signai transduction pathways. 
THE ROLE OF INTERLEUKIN 2(IL2) IN THE REGULATION OF ILZR $\alpha$ GENE EXPRESSTON IN A T CELL CLONE, S. Barangé 1, P.Romero ${ }^{2}$ and $M$. Nabholz ${ }^{1}$. 'ISREC and Ludwig Institute for Cancer Research.

CSF12 is an IL2 dependent murine $\mathrm{CD}^{+}{ }^{+} \mathrm{T}$ cell clone which resembles normal $T$ cells. Stimulation of CSF12 cells by antigen (Ag) or anti-CD3 MAbs, in presence of IL2, induces, successively, the appearance of IL2R $\alpha$ MRNA, an increase of IL2R $\alpha$ chains on the cell surface, entry into the cell cycle and an increase in viable cell numbers. We have studied the role of IL2 versus $\mathrm{Ag}$ in the regulation of IL2Ro gene expression. In a first stage Ag induces IL2Ra gene transcription independently of IL2. This response is partially resistant to inhibition of protein synthesis. In the absence of IL2 it is transient; transcripts are no longer detectable after 24 hours. IL2 induces a second stage characterized by a further increase of ILZRa mRNA levels, which peak at 48 hours and remain detectable for $5-6$ days. Transient transfection experiments show that IL2R $\alpha$ expression depends on cis-acting elements between -802 and -1972 of the mouse II 2 Ra $5^{\prime}$ flanking region.

\section{S14-14}

Increased Resistance to Endotoxic Shock in Interferon- $\gamma$ Receptor Deficient Mice

Bruce D. Car, Vicki M. Eng, Bruno Schnyder, Marietta Weber, Laurence Ozmen+, Sui Huang*, Michel Aguet*, Bernhard Ryffel ${ }^{1}$ )

Institute of Toxicology of the Swiss Federal institute of Technology and University of Zürich, 8063 Schwerzenbach, +Pharmaceutical Research, Hoffmann-La Roche, Basel and "Molecular Biology, University of Zürich, Hönggerberg, Zürich, Switzerland.

Although tumor necrosis factor (TNF) is established as the central mediator of endotoxic shock, interferon- $\gamma$ (IFN $\gamma$ ) clearly also functions in a toxic fashion. The advent of IFN $\gamma$ receptor negative (IFN $\gamma \mathrm{R}-/ \cdot$ ) mutant mice has enabled a direct assessment of the role of IFN $\gamma$ in endotoxin-induced shock. We report that IFN $\mathrm{HA}-1$ - mice have an increased resistance to lipopolysaccharide-induced toxicity (LPS). LPS-induced lymphopenia, thrombocytopenia and weight loss seen in wild type mice were attenuated in IFNyR- $\%$ - mice. IFNyR- - mice survived in the D-galactosamine-LPS model IFNyR-/- mice. IFNyR-/- mice survived in the D-galactosamine-LPS model
when conditions were $100 \%$ lethal for wild type mice, correlating with serum when conditions were $100 \%$ lethal for wild type mice, correlating with serum
TNF levels of up to 10 fold higher in wild type mice. Bone marrow and splenic macrophages from IFN $\gamma R-$ - mice had a 3 to 5 fold decreased LPSbinding capacity, with serum from these mice lowering macrophage LPSbinding by a further $50 \%$. Thus, depressed TNF synthesis, diminished expression of LPS receptors and low plasma LPS-binding capacity in the expression of LPS receptors and low plasma LPS-binding capacity in the
mutant mice likely combine to manifest in the resistant phenotype of IFN $\gamma$ R-/- mice to endotoxin.

\section{S14-15}

A TWO-STAGE MODEI, FOR THE TRANSCRIPTIONAI, REGULATION OF THE INTERLEUKIN-2 RECEPTOR $\alpha$ (II,2Ra) GENE

Sperisen, P., Wang, S.-M., Reichenbach, P. Corthésy, P. and Nabholz, M. ISREC, Epalinges. The capacity of $T$ lymphocytes to proliferate in response to IL2 is controlled, at least in part, by the expression of the IL2Ra gene. Regulation of this gene occurs mainly at the level of transcription. Analysis of the kinetics and inhibitor sensitivity of ILZRa induction in a rodent $T$ cell line, and transient transfection studies with mouse IL2R 5 'flanking region/ $\beta$-globin reporter gene constructs shows that induction of expression occurs in two phases, an immediate early response that depends on cis- acting elements between nt - 584 and -55 , which is followed by a second phase that depends on stimulation with IL2 and is mediated by cis-acting elements between nt - 1389 and - 1306 . Experiments with normal. $T$ cells suggest that this biphasic regulation is a general feature of IL $2 R \alpha$ expression.
TRANSCRIPTIONAI REGULATION OF THE IL2R G GENE: A STUDY IN TRANSGENIC MICE.

Soldaini, E.,1 Beermann, F.,1 Corthésy, P., 1 MacDonald, H.R., 2 Nabholz, M. 1

IISREC and 'LICR, 1066 Epalinges.

A fragment consisting of $2.7 \mathrm{~kb}$ of the mouse IL2Ra gene 5'flanking region linked to the CAT gene was used to generate 5 independent transgenic (tg) mouse lines. Tissue analysis showed CAT activity in the thymus of 4 lines, in the bone marrow of 2 lines and in the spleen of 1 line. No CAT activity was detected either in lymph nodes or in non-lymphoid tissues from any line. Transgene expression was inducible in mature $\mathrm{T}$ cells by stimuli (ConA+IL2) which induce IL2Ra expression. Maximal expression of both the IL2R $\alpha$ and the trangene depended on stimulation by IL2. In CD4-CD8- thymocytes suboptimally stimulated with PMAtionomycin IL1 and IL2 induced both IL2Ra and CAT expression. These results show that the regulatory region studied contains many of the cis-acting elements responsible for the tissue specific expression and inducibility of the IL2R gene.

S14-17

MAPPING OF LYMPHOCYTE SPECIFIC CONSTITUTIVE AND INDUCIBLE DNASEI HYPERSENSITIVE SITES IN THE MOUSE IL2R 5 'FLANKING REGION

Pla, M, Espel, E. and Nabholz, M.

ISREC, 155, ch. des Boveresses, 1066 Epalinges Previous work from our laboratory has identified cis-acting elements that regulate transcription of the mouse gene coding for the . a chain of the high affinity II2 receptor (IL2R $\alpha$ ), by transient transfection studies. In a complementary approach we have screened the $5^{\prime}$ portion $(-7.8$ $\mathrm{kb} /+4.1 \mathrm{~kb})$ of the gene for tissue specific and/or inducible DNaseI hypersensitive sites. In chromatin from fibroblasts or kidney epithelial cells this segment of the II $2 R a$ gene does not contain any DNaseI hypersensitive sites. In contrast, in resting $\mathrm{T}$ - and B-lymphocytes, as well as in activated T cells, two sites (DH1, $0.05 \mathrm{~kb}$; $\mathrm{DH} 3,-5.8 \mathrm{~kb}$ ) were found. A third site (DH2, $-1.35 \mathrm{~kb}$ ) is detectable in $\mathrm{T}$ cells that have been activated with concanavalin $A$ and IL2. This site maps to the same position as cisacting regulatory sequences that are required for the response of the IL $2 R \alpha$ gene to signals from the II2 receptor.

\section{$\mathrm{S} 14-18$}

IL- 1 signal transduction to IL- 6 in human fibroblasts is modulated by serum and by growth factors

W. Liu, Y. Cao and U. N. Wiesmann, Department of Pediatrics, University of Berne, Switzerland

Interleukin-6 (IL-6) production in cultured human dermal fibroblasts can be stimulated by interleukin-1 (IL-1) added to the culture medium. Absence of fetal calf serum and of growth factors (insulin, EGF, T3) reduced the stimulation by more than $50 \%$. EGF and $\mathrm{T} 3$ and even more choleratoxin increased the IL-6 production in absence of fetal calf serum. The effect was dose dependent. 2\% human $A B$ serum substituted for $10 \%$ fetal calf serum. pretreatment of the cultures with serum or growth factors prior to stimulation with IL-1 had a stimulatory effect (serum, $T 3, E G F$ ) or an inhibitory effect (hydrocortisone. choleratoxin). Our results suggest that the IL-1 signal transduction to IL- 6 is modulated by serum components and growth factors as well as through C-AMP produced by choleratoxin. 
S14-19

COCULTURES OF T-CELLS AND HUMAN DERMAL FIBROBLASTS PRODUCE LARGE AMOUNT OF INTERLEUKIN-6 B. Spörri ${ }^{1}$, ER Wältit ${ }^{2}$, A. Limat ${ }^{3}$, T. Hunziker ${ }^{3}$, UN Wiesmann ${ }^{1}$ Department of Pediatrics ${ }^{1}$, Pathology ${ }^{2}$, Dermatology ${ }^{3}$, University of Berne.

Interleukin- 6 (IL-6) is produced by a variety of cells and plays a central role in host defense mechanisms. Its function include in duction of IL-2 and IL-2 receptor expression, proliferation and differentiation in T-cells. In cocultures of human dermal fibroblasts and allogenic human T-cells a cell number dependent 10 to 100 fold increase of the IL-6 production could be demonstrated after $24 \mathrm{~h}$ and $48 \mathrm{~h}$. Conditioned medium from $\mathrm{T}$. cells and from fibroblasts failed to induce IL-6 secretion in fibroblast and T-cell cultures, respectively. No up-regulation of IL-6 production was found in cocultures of fibroblasts with $\mathrm{T}$. cells killed by ethanol and in T-cell culture incubated with recombinant $h \mathrm{IL}-1 \alpha$. After separation of the cells IL- 6 production in fibroblast and T-cells returned to precoculture levels. Our results indicate that cell-cell contact more than soluble factors must be involved in the up-regulation of IL-6 synthesis in cocultures of T-cells and fibroblast. Using cocultures of autologes human T-cells and fibroblasts, we are currently investigating whether the cell contact essential for the IL-6 production depend on immunolcical interactions of $T$-cells and fibroblasts.

\section{$\mathrm{S} 14-20$}

\section{High-Level Expression of Interleukin 8 (IL-8) in Cells Surrounding} Necrosis in Human Glioblastomas

Desbaillets I., Diserens A.-C., Hamou M.-H., Tada M., de Tribolet N, Van Meir E. Laboratoire de Neurochirurgie, CHUV, Lausanne.

Rapid growth of tumor often results in oxygen and nutritional deprivation leading to extensive necrosis, which is one of the characteristics of glioblastomas (Gbl). Neoplastic cells surrounding necrotic areas often present a peculiar arrangement of cells called "pseudo-palisading" - as a hallmark of this entity, together with abundant neovascularization. A special biological milieu is probably formed in these palisading areas by certain unknown cytokines and/or growth factors induced by the necrotic process. Plate et al. (1992) reported the expression of vascular endothelial growth factor (VEGF) in the palisading cells. We previously demonstrated that $\mathrm{Gbl}$ cells produce IL-8, which is known to be an angiogenic factor. The aim of this study is first to assess if IL-8 is expressed in the palisading cells, and second to determine if hypoxia can trigger IL-8 gene expression. In vivo observation using in situ hybridization and immunohistochemistry showed that IL-8 is highly expressed specifically in the palisading cells. We also demonstrated the mRNA expression of IL-8 receptor in the endothelial cells adjacent to necrotic area. To simulate the in vivo condition, two in-vitro models are currently developed to determine if $I L-8$ is induced by hypoxic insult on cells. First, Gbl cell lines are cultured in the absence of oxygen and IL-8 expression is assessed by Northern blot analysis. Preliminary results have demonstrated the induction of IL-8 by hypoxia. Secondly Gbl cells are grown in spheroids until development of central necrosis. The IL-8 expression will be assessed by in situ hybridization combined with immunohistochemistry to determine if the cells surrounding necrosis express IL-8.

\section{S14-21}

Phenotype of Mice with Targeted Disruption of the Gene for Double-Stranded RNA-Dependent Protein Kinase

Yi-Li Yang, Luiz Reis, Adriano Aguzzi*, Reinhold Schaefer*, Michel Aguet and Charles Weissmann. Institut für Molekularbiologie I Universität Zürich, 8093, *Departement Pathologie, Universitätsspital, 8091 Zürich, Switzerland

Double-stranded RNA-dependent protein kinase (PKR) is induced by type I interferon (IFN) and is implicated in the establishment of the antiviral state. Upon activation, PKR phosphorylates the eukaryotic initiation factor EIF-2, thereby throttling protein synthesis. It was suggested that PKR may be essential for the induction of IFN- $\beta$ gene expression by both virus and double-stranded RNA and for the activation of at least some IFNinducible genes. Recently it was proposed that PKR is a tumor suppressor gene because 3T3 cells overexpressing inactive human PKR gave rise to tumors in nude mice (Koromilas et al., 1992; Meurs et al., 1993).

We have produced homozygous PKR knockout ( $\mathrm{PKR} / \%)$ mice and found that they develop normally and have no striking phenotype. Induction of IFN- $\alpha$ and IFN- $\beta$ gene transcription by virus was unimpaired in fibroblasts derived from $\mathrm{PKR}^{\mathrm{o} / \mathrm{o}}$ mice, as was the induction of several IFN-stimulated genes. So far, no spontaneous tumor formation was observed. $\mathrm{PKR}^{\mathrm{o} / 0}$ embryonal stem cells showed no increased malignancy in nude mice as compared to their wild type counterparts. We conclude that PKR does not play an essential role in viral induction of the IFN genes and that its tumor suppressing effect, if any, is redundant.
S14-22

Interferon is Fully Inducible in Mice Devoid of Interferon Regulatory Factor 1 (IRF-1)

Luiz Reis, Heinz Ruffner, Michel Aguet and Charles Weissmann. Institut für Molekularbiologie I, Universität Zürich, 8093 Zürich, Switzerland

It has been proposed by Taniguchi and his colleagues that activation of the interferon (IFN)- $B$ gene by virus or double-stranded RNA involves induction and modification of IRF-1. Overexpression of IRF-1 can induce expression of the IFN-B gene in certain cells. A role of IRF-1 in the activation of IFN- $\alpha$ genes has also been proposed. Furthermore, IRF-1 has been claimed to play the role of a tumor suppressor gene.

We have generated mice in which both IRF-1 alleles were disrupted and found them to develop normally. No spontaneous tumors have been observed so far. Injection of poly $(\mathbf{I}) \cdot \operatorname{poly}(\mathrm{C})$ resulted in the same levels of IFN in blood of wildtype and null mice, and equal IFN- $\alpha$ mRNA levels in spleen. Induction of wild type and IRFo/o embryo fibroblasts (MEFs) with virus resulted in the same levels of IFN- $\alpha$ and IFN- $\beta$ mRNA respectively. In the case of poly(I)-poly(C) induction, the levels of both IFN mRNAs were higher in wild type than in mutant cells; this difference was abolished if the cells were first primed with type I IFN. We conclude that IRF-1 does not play an essential role in the induction of IFN genes.

\section{S14-23}

Cytokine Network in Human Glioblastomas - A Primary Role of Interleukin 1 Autocrine Mechanisms.

Mitsuhiro TADA, Annie-Claire Diserens, Isabelle Desbaillets, MarieFrance Hamou, Nicolas de Tribolet, Erwin Van Meir; CHUV, Lausanne There is increasing evidence that a variety of cytokines produced by glioblastoma (Gbl) cells modulate the tumor growth by affecting the host's immune response and by stimulating neovascularization. Cytokines such as IL-1, IL-6, IL-8, MCP-1, IL-10 and TGF- $\beta 2$, affect each other's production and receptor system and seem to form a cytokine network. Among them, IL-1 may play a pivotal role, since IL-1 can induce or increase the production of other cytokines (IL-6,IL-8,MCP-1,TGF- $\beta 2$ ) and modulate their receptor expression. To test this hypothesis, we investigated co-expression of cytokines and their receptors in $15 \mathrm{Gbl}$ cell lines and 15 Gbl tissues using RT-PCR, Northern blot analysis, immunohistochemistry and ELISA quantification. A majority of the Gbl cell lines and Gbl tissues expressed IL-1 $\alpha$, IL-1 $\beta$, type I and type II receptors, indicating the presence of an IL-1 autocrine loop. IL-1 stimulated growth of some of the cell lines. A positive correlation of IL-6 and IL-8 expressions with the presence of an IL-1 autocrine loop in the cell lines was found. Immunohistochemical studies showed the in vivo co-expression of the IL-1 family and the secondary cytokines (IL-6, IL-8) in Gbls. Antisense oligonucleotide to IL-1 $\alpha$ and/or IL-1 $\beta$ partly suppressed this secondary cytokine expression. These results demonstrate that the IL-1 autocrine loop plays a central role in the formation of the cytokine network in Gbls.

\section{S14-24}

TRANSGENIC MICE EXPRESSING HUMAN SOLUBLE TNF RECEPTOR 1 ARE HIGHLY SENSITIVE TO LISTERIA MONOCYGENES, MYCOBACTERIUM TUBERCULOSIS, AND LESHMANIA MAJOR INFECTIONS

1. Garcia, K. Araki, Y. Miyasaki, G. Grau, W. Lesslauer, G. Marchal, G. Milon, and P. Vassalli, Depart. of Pathology, CMU, University of Geneva, Hoffmann-La Roche, Basel, Switzerland, Institut Pasteur, Paris, France.

Seven lines of mice expressing a soluble TNF-R1 fused to a human IgFc placed under control of $\alpha$-1-anti-trypsin promoter were established. Depending on the mouse line, the level of transgene product in the blood ranged from $25 \mathrm{ng} / \mathrm{ml}$ to 150 $\mathrm{ug} / \mathrm{ml}$. Mice with high expression level of transgene have no activation of bioactive TNF after LPS injection and were completely resistant to septic chock while mice with low transgene expression were partially resistant. In the other hand mice with high blood levels of STNF-R1 are very susceptible to $L$. monocytogenes and $M$. tuberculosis and die after infection of sublethal dose. Transgenic mice with genetic bacground resistant to infection inoculation with Leshmania major results in lesions unable to heal. Our data show that high levels of soluble receptors are required to block TNF endotoxin lethal toxicity and that TNF activity is necessary for host immune defense against bacteria and parasitic diseases. 
S14-25

TGF- $\beta$ INHIBITS THE CHEMICALLY INDUCED MITOGENIC RESPONSE IN CULTURED RAT HEPATOCYTES

R.Fasciati, K.Ohno, B.Saad, B.Ryffel and P.Maier, Institute of Toxicology, ETH and University of Zürich, $\mathrm{CH}-8603$ Schwerzenbach

TGF- $B$ plays an important role as a negative regulator of hepatocyte proliferation in liver regeneration. Exposure of rodents to nongenotoxic carcinogens like Cyproterone Acetate (CPA), Thioacetamide (TA) and Phenobarbital (PB) causes abnormal hepatocyte proliferation and liver tumors after long term treatment. This suggests that these chemicals have either a direct mitotic activity or impair the negative regulatory system for growth.

Therefore the inhibitory activity of TGF-B on DNA-synthesis induced whith CPA was investigated in cultured rat hepatocytes. After a $48 \mathrm{~h}$ exposure to CPA, a dose dependent increase in DNA synthesis ( ${ }^{3} \mathrm{H}-\mathrm{TdR}$ incorporation) was observed. The maximum effect was attained with $12 \mu \mathrm{M}$ CPA (up to 4.3 fold) which was stronger than with $10 \mathrm{ng} / \mathrm{ml} \mathrm{EGF}$ (up to 2.8 fold). TGF- $B_{1}$ and TGF- $B_{2}$ inhibited this response dose dependently (ID50 $=0.1 \mathrm{ng} / \mathrm{ml}$ ) and reduced it to control levels at $1 \mathrm{ng} / \mathrm{ml}$. These findings show that the TGF- $B$ mediated pathway for negative growth control in hepatocytes is still responding after $\mathrm{CPA}$ treatment.

\section{S14-26}

MX1 INHIBITS INFLUENZA A VIRUS ImRNA SYNTHESIS AT THE LEVEL OF ELONGATION

H. Landis, H. P. Hefti, C. di Paolo, and J. Pavlovic, Instítut für Medizinische Virologie der Universităt Zürich, $\mathrm{CH}-8028$ Zürich

Mouse Mxt protein is an interferon (IFN) induced GTPase with intrinsic antiviral activity against influenza $A$ viruses. It accumulates in the cell nucleus and inhibits the transcription of influenza virus genes. Recently, we have shown that $M \times 1$ exerts its inhibitory activity by interfering with the function of the viral polymerase subunit PB2. PB2 is responsible for recruiting $5^{\prime}$ ends of cellular mRNAs as primers and for the elongation of nascent viral transcripts. To elucidate which PB2 dependent step of viral mRNA synthesis is the target of $M \times 1$ action, we examined the activity of recombinant $M \times 1$ protein in an in vitro transcription system of influenza virus. First, we expressed wildtype and mutant Mx1 proteins, carrying a histidinetag at their N-terminus, in $E$. coll and purified them under nondenaturing conditions by $\mathrm{Ni}$-chelate affinity chromatography. $\mathrm{M} \times 1$ efficiently inhibited viral transcription in vitro, while mutant $M \times 1$ proteins, lacking antiviral activity in vivo, were inactive. Moreover, the use of capped synthetic RNA primers revealed, that Mx1 almost completely abrogated the elongation of viral transcripts, whereas the the cap-binding and endonuclease activity of PB2 was not affected.

\section{S14-27}

\section{Characterization of a murine tumor necrosis factor $\alpha$ -} LacZ reporter construct

Aregger, H., Eugster, H.P., and Ryffel, B., Inst. of Toxicology., Swiss Fed. Inst. of Technology, Schorenstr. 16, CH-8603 Schwerzenbach.

Tumor necrosis factor $\alpha$ (TNF $\alpha)$ is a prominent mediator of a variety of different pathologies such as endotoxic shock syndrom and rheumatoid arthritis. It also plays a crucial role in host defence against bacterial and viral infection. Up to now, only few data about signal pathways leading to TNF $\alpha$ induction are available. An easy to handle TNF $\alpha-\mathrm{LacZ}$ reporter assay will represent a useful tool to study signal transduction on the one hand and provide data about compounds with potential TNFo inducible activity on the other hand.

In order to develop a murine macrophage cell line capable to easily report TNF $\alpha$ induction, different TNF $\alpha$-LacZ reporter constructs were assembled. Data about functionality of the different constructs in murine macrophages will be presented in the context of TNF $\alpha$ expression.
$514-28$

Neutralization rates of TNF- $\alpha$ from eight animal species by a monocional antibody against human TNF: implication for receptor action?

U. Pauli, G. Bertoni, K. Rohrbach, B. Suter and E. Peterhans, Institut für Veterinär-Virologie, Universität Bern, $\mathrm{CH}$ - 3012 Bern

We have recently developed a sensitive bioassay for measuring porcine TNF- $\alpha$ based on homologous PK(15) cells. This bioassay is 100 - to 1000 -fold more sensitive than the widely used L929 bioassay. Also, human TNF- $\alpha$ and human TNF-B were detected with a slightly higher sensitivity in the new test compared to the L929 bioassay. We now show that also canine ovine, bovine, equine and caprine TNF- $\alpha$ can be detected with the PK(15) bioassay. We tested a murine monoclonal anti-human TNF- $\alpha$ antibody for its capacity to neutralize TNF- $\alpha$ from eight different animal species. The action of this antibody revealed that neutralization of TNF bioactivity is a complex phenomenon, indicating species-specific differences in the interaction with TNF receptors. To study this differential neutralization, we are cloning the porcine TNF receptors for expression in a heterologous cell system.

Expression of type $A$ and type $B$ tumor necrosis factor (TNF) receptors on human melanoma cells and their possible regulation by interferon $\mathrm{g}$ and dibutyric c-AMP. Carrel S., Salvi S., Hartmann F., Schreyer M. \& Rimoldi D. Ludwig Institute for Cancer Research, Lausanne Branch, $\mathrm{CH}$-1066 Epalinges, Switzerland

Administration of high dose $\mathrm{r}-\mathrm{TNF} \alpha$ in combination with IFN $\gamma$ in isolation and perfusion of the limbs in melanoma patients has shown to be very promising with a response rate greater that $80 \%$. This observation prompted us to investigate the possible expression of the TNF receptors in melanoma cells using the monoclonal antibodies UTR 1 specific for the TNF Type A $(55 \mathrm{kDa})$ receptor and HTR-9 specific for the TNF Type $B(75 \mathrm{kDa})$ receptor. Flow cytometric analysis of cultured melanoma cells showed the presence at low level of the TNF Type A and to a slightly higher level of the Type B receptor. Similar results were obtained in vivo by immunohistochemistry on fresh tumor material. Treatment of melanoma cells in culture with dbc-AMP induced up to a 2 fold increase in the number of Type A TNF receptors with only a minimal change in the number of Type $B$ receptors. This increased expression of TNF receptors is confirmed by direct binding experiments using ${ }^{125}$ I-labeled TNF $\alpha$. The number of CPM's bound on dbc AMP treated cells was about $0.5-3$ fold higher than on untreated control cells, likewise incubation of melanoma cell lines with IFN $\gamma$ increased the specific binding of subsequently added ${ }^{25} \mathrm{I}$-labeled TNF $\alpha$. From flow cytometric analysis using the two anti-TNF receptor antibodies it became evident that IFN $\gamma$ was able to increase the expression of both Type A and B receptors depending on the cell line used.

\section{S14-30}

CLONING OF A NOVEL PROTEIN BINDING TO LYMPHOKINE, FOS AND MYC MRNA WITH UNEXPECTED ENZYME ACTIVITY $\mathrm{J}$. Nakagawa, H-P. Waldner, S. Meyer-Monard, and $\mathrm{Ch}$. Moroni

Inst. Medizinische Mikrobiologie, Univ. Basel

An AU-rich sequence in the 3' untranslated region of lymphokines, $\mathrm{c}$-fos, and $\mathrm{c}$-myc proto-oncogene mRNA is responsible for their rapid decay. We have purified a $32 \mathrm{kd}$ protein by an AUUUA affinity column from human brain. Partial arnino acid sequence was obtained and the corresponding CDNA has been cloned. Unexpectedly the cDNA sequence exhibited significant homology to enoyl CoA hydratase and bacterially expressed recombinant protein indeed showed the activity. While rat hydratase did not show RNA binding activity, the recombinant protein bound to cfos, c-myc, AUUUA cluster of IL-3 mRNA, and Adeno virus IV, but not to mutated Ad-IV, nor irrelvant transcript. The binding was competed out by poly $U$. Interestingly the protein seems to be processed from a larger precursor. We suspect that the protein plays a role in mRNA turnover and perhaps in oncogenesis. 
S14-31

GROWTH INHIBITION AND POSTTRANSCRIPTIONAL DOWNREGULATION OF IL-3 MRNA BY CYCLOSPORIN A AND RAPAMYCIN IN AUTOCRINE MAST CELL TUMORS

Rolf Banholzer, Asha P.K. Nair, Sinuhe Hahn and Christoph Moron Institute for Medical Microbiology, University of Basel

In $T$ cells, the immunsuppressive agent CsA is known to inhibit $\mathrm{Ca}_{2} 2+$ dependent induction of lymphokine mRNA transeription. In contrast, the immunsuppressive drug rapamycin inhibits the lymphokine induced proliferation. We have analysed the effect of these drugs on a $\mathrm{v}$-H-ras induced autocrine mast cell tumor line which expresses IL-3 constitutively. CsA and rapa inhibited autocrine growth by different mechanisms, , because added IL-3 could antagonize inhibition by CsA, but not by rapa. Whereas CsA acted by downregulation of IL-3 mRNA expression, rapa blocked IL-3 induced proliferation. CsA also inhibited IL-3 superinduction by Ca-ionophore, whereas rapa did not. Nuclear run on assay indicated that the mechanism is posttranscriptional. Therefore, we have introduced IL-3 transgenes with and without the AU-rich region in the $3^{\prime}$ UTR of the mRNA into a tumor line. In contrast to the wild type, the expression of the mutant construct was insensitive to CSA. Since the mutant lacks sequences known to regulate rapid mRNA decay, we suggest that CsA affects the regulation of IL-3 mRNA stability.

\section{S14-32}

Generation of Interleukin-3 (IL-3) autocrine mast cell tumors by $\mathrm{v}-\mathrm{H}$-ras involves transcriptional or posttranscriptional alterations in the control of IL-3 expression

Hans H. Hirsch, Asha P.K. Nair, and Christoph Moroni,

Institute for Medical Microbiology, University of Basel, Petersplatz 10, CH-4003 Basel.

Activating mutations in the ras oncogene are not sufficient to induce malignant transformation, but require further cooperating alterations. Expression of the v-H-ras oncogene in the IL-3 dependent PB-3c mast cells leads to the generation of two classes of IL-3 autocrine tumors in vivo. Autocrine IL-3 expression in class-1 tumors results from increased IL-3 mRNA stability due to the loss of negative trans-acting posttranscriptional control. This defect can be corrected by somatic cell fusion to the nontumorigenic parental PB-3c resuiting in downregulation of oncogenic IL-3 expression and concomitant tumor suppression. Expression of the $\mathrm{v}$-H-ras oncogene remains unaffected in class-1 hybrids. In contrast, class-2 tumors are characterized by transcriptional activation of the IL-3 gene due to the insertion of an endogenous retroviral element (intracisternal A-particle) which cannot be overcome by cell fusion (see Hirsch et al., 1993, J.Exp.Medicine 178, 403-411). Although $\mathrm{v}$ - $\mathrm{H}$-ras is required for generation of either class of tumors, expression of anti-sense ras constructs inhibited only the proliferation of class-1 tumor cells. Experiments are underway to characterize the target and the nature of the class-1 alteration.

\section{S14-33}

\section{GROWTH REGULATION MEDIATED THROUGH THE IL-4 RECEPTOR IN HUMAN COLONIC CARCINOMA CELLS B. Schnyder H. Lahm, A. Yilmaz, B. Ryffel and B. Car. Institute of Toxicology, ETH and University of Zürich, $\mathrm{CH}$ - 8603 Schwerzenbach}

Interleukin-4 (IL-4) is a T-cell derived lymphokine which, in addition to its effects on the immune system, is also able to suppress the growth of certain tumor cells. IL-4 reduced the growth rate of four human colon tumor cell lines up to $70 \%$ in a dose-dependent manner. Three other cell lines showed no significant alteration of $3 \mathrm{H}$-thymidine incorporation or colony formation in the presence of $100 \mathrm{u} / \mathrm{mI} \mathrm{IL-4}$. Responder and nonresponder tumors were immunopositive for IL-4 receptor (IL$4 R$ ) expression in flowcytometric analysis, using monocional antibodies raised against recombinant $\mathrm{IL}-4 \mathrm{R}$ and bound biotinyated IL-4. Responsive tumors expressed more receptors. $\mathrm{IL}-4$ binds with high affinity to a $130 \mathrm{kDa}$ transmembrane receptor (IL-4R), through which the extracellular signal has been shown to be transduced. Coimmunoprecipitation and chemical crosslinking studies have enabled us to demonstrate IL-4R target proteins. Tyrosine phosphorylation of various proteins between 70 and $170 \mathrm{kDa}$ appears to be initiated during IL-4mediated signaling events in colon tumor cells. The cloning of human IL-4R target proteins will contribute to the understanding of the IL-4 signal transduction pathway at the initial stage.
S14-34

Expression of sten cell factor (SCF) gene by human neuroblastoma (NB) cells. Beck D., Gross N. Beretta C. \& Perruisseau G. Onco-hematology unit, Department of Pediatrics, University Hospital, Lausanne, Switzerland. Some non-hematopoietic cell lines produce both SCF and its receptor, the c-kit protein (c-kit). Testing the hypothesis that $\mathrm{SCF}$ may be involved in secondary growth of NB bone marrow metastasis, we found and previously communicated (Beck D. et al, Proc AACR, 34, 52, 1993) a low or absent expression of c-kit in NB cells. The mRNA and surface expressions of SCF gene in NB cell lines
grown in chemically-defined medium were evaluated by northern blot analysis and flow cytometry. The SCF antigenic concentrations in culture supernatants were determined by ELISA and growth-inhibition experiments performed by pre-incubating NB cells with anti-SCF antibodies prior to $3 \mathrm{H}$-thymidine uptake assay. The SCF mRNA was expressed in 4 of 5 tested lines but no membrane-bound antigen could be detected on those cells. Detectable levels of SCF in the supernatants were found in $5 / 7$ lines (range : $39-96 \mathrm{pg} / \mathrm{mL}$ ). Blocking experiments resulted in a decrease of DNA gynthesis in l out of 7 lines only. From these and previous results, we conclude that SCF is synthesized and released by many NB cells in vitro but the functional role of it remains undetermined since SCF does not appear to be usually involved in autocrine or paracrine growth stimulation of NB cells (supported by Swiss FNRS grant 3200-031005).

\section{S14-35}

ROLE OF NITRIC OXIDE IN REGULATING RENAL HEMODYNAMICS IN NEWBORN RABBITS

L. Ballèvre, M. Thonney and J.P. Guignard, Service de pédiatrie, CHUV, Lausanne, Switzerland

Nitric oxide (NO) promotes vasorelaxation of renal vessels in vitro. The purpose of the present study was to assess the role of NO in regulating renal hemodynamics in vivo. Renal blood flow (RBF) and glomerular filtration rate (GFR) were studied in anesthetized mechanicallyventilated newborn rabbits both before and after the administration of a NO synthesis inhibitor, $\mathrm{N}^{\mathrm{G}}$-nitro-Larginine methyl ester (L-NAME), at a dose of $50 \mu \mathrm{g} / \mathrm{kg}$ followed by $10 \mu \mathrm{g} / \mathrm{kg} \times \mathrm{min}$. Such a dose of $\mathrm{L}-\mathrm{NAME}$ did not modify mean arterial pressure or pulse rate. $B y$ contrast, the renal vascular resistance increased by $31 \pm$ $9 \%(P<0.005)$ while RBF decreased by $20 \pm 6 \%(\mathbf{P}<$ $0.02)$. GFR and urine flow rate remained constant. The overall results suggest that in normal conditions NO may play a role in decreasing the renal vascular resistance of the immature kidney, without altering systemic blood pressure.

\section{\$14-36}

RENAL AND SYSTEMIC EFFECTS OF ENDOTHELIN-1 IN NEWBORN RABBITS

D. Semama, J.-B. Gouyon, J.-P. Guignard, Service de pédiatrie, CHUV, Lausanne, Switzerland

The renal effects of endothelin-1 were investigated in 16 anesthetized and mechanically-ventilated newborn rabbits. Each animal acted as its own control. In 8 newborn rabbits, a bolus injection of 5 nmol.kg-1 of endothelin-1 caused an initial fall in mean blood pressure (MBP) followed by a gradual but significant increase in MBP that lasted for 45 $\mathrm{min}$. The dramatic increase in renal vascular resistance $(+28$ $\pm 4 \%$ ) induced by endothelin led to a fall in glomerular filtration rate $(-12 \pm 4 \%)$ and renal blood flow (-16 \pm $3 \%$ ). In spite of the reduction of GFR and RBF, urine flow and sodium excretion rates increased significantly $(+20 \pm$ $5 \%$ and $+49 \pm 9 \%$, respectively). In 8 additional newborn rabbits, a bolus injection of 1 nmol.kg-1 of endothelin-1 - a dose that usually induces marked renal and systemic vasoconstriction in adult models - did not affect systemic or renal hemodynamics. In conclusion, endothelin induces renal and systemic vasoconstriction, and affects water and sodium homeostasis during the neonatal period. However, these effects occur under higher doses than those used in adult animals, possibly reflecting receptor immaturity and/or interference of high levels of counteracting hormones. 
C. Maake ${ }^{1}, W$. Hanke ${ }^{2}$ and M. Reinecke ${ }^{1},{ }^{1}$ Institute of Anatomy, University of Zürich, Switzerland and 'Department of Zoology II, University of Karlsruhe, F.R.G.

The pancreatic islets of anurans show insulin (INS), glucagon (GLUC), somatostatin (SOM) and pancreatic polypeptide (PP) containing cells. Since only limited information exists on the ontogeny and on the presence of insulin-like growth factor 1 (IGF1), we studied the development of the gastro-entero-pancreatic (GEP) system in Xenopus laevis using immunohistochemical techniques. Singular iNS-immunoreactive (-IR) cells were observed in the pancreas as early as on stage 41 . At stage 43 , the first PP-IR cells were found in the GEP system followed by SOMIR and GLUC-IR cells. The first pancreatic islets consisting of INSIR and GLUC-IR cells occurred around stage 50 . Between stage 54 and 60 , i.e. after the onset of metamorphosis, the endocrine cells decreased in number and the islets partly disintegrated. Starting with stage 61 , the islets reformed and the amount of endocrine cells increased reaching the adult status at stage 63 . Around stage 52, IGF-1-immunoreactions appeared. IGF-1immunoreactivity was found in PP-IR and GLUC-IR cells but not in INS-IR and SOM-IR cells. It is assumed that islet-derived IGF-1 may play an important role during metamorphosis in Xenopus. Supported by the Swiss National Foundation (grant 32-33349.92)

\section{Membrane Proteins: Structure and Function}

\section{S15-01}

THREE AUGS AND POSSIBLY TWO SIGNAL SEQUENCES GIVE RISE TO FUNCTIONAL ISOFORMS OF A VIRAL TYPE I TRANSMEMBRANE PROTEIN

T. CATHOMEN AND R. CATTANEO, Institut für Molekularbiologie I, Universität Zürich, Hönggerberg, $\mathrm{CH}-8093$ Zürich

The signals used for synthesis of the measles virus fusion $(F)$ protein are poorly understood: a long ( $>570$ bases) untranslated region is followed by three in frame AUGs at codons 1,4 and 15 . $F$ is a type I transmembrane protein with a postulated signal sequence of 31 amino acids. We confirmed that the 46 aminoterminal residues contain a signal peptide by transfering them to another protein. With the other envelope protein hemagglutinin, $F$ protein induces virus-cell and cell-cell fusion. Mutagenesis of the three AUGs indicates that proteins initiated at codon 1,4 or 15 are all functional, but show slightly different fusion efficiencies. Forms transiated from AUG 1 or 4 appear larger in electrophoresis than forms initiated at AUG 15, suggesting that two different signal peptide cleavage sites are used. We are currently investigating whether both $F$ protein forms are incorporated in viral particles. The production of protein isoforms with staggered amino-termini is common in cytoplasmic and type II transmembrane proteins, but signal sequences usually preclude a similar organization of type I transmembrane proteins.

\section{S15-02}

The heat-stable antigen (HSA or mouse CD24) gene is differentially regulated but has a housekeeping promoter.

Roland H. Wenger*, Georges Köhler and Peter J. Nielsen. Max Planck Institut für Immunbiologie, Stübeweg 51, D-79108 Freiburg i. Bsg. Winterthurerstrasse 190, $\mathrm{CH}$-8057 Zürich.

Expression of the GPI-anchored murine glycoprotein heat-stable antigen (HSA) shows tissue-specific as well as developmental regulation. During the maturation of several hematopoietic lineages, or absent in terminally differentiated celis. We present evidence suggesting that this regulation of the HSA gene (Cd24a) occurs at the transcriptional level. In addition, sequence and methylation analysis of the Cd24a promoter revealed characteristics of both "housekeeping" and tissue-specific promoters, including a methylation-free, Hpall Tiny Fragment (HTF) island, multiple putative $\mathrm{SP} 1$ and $\mathrm{AP}-2$ consensuS binding sites and a TATA box. Functional analysis of a $0.6 \mathrm{~kb}$ DNA fragment containing these elements fused to the CAT reporter gene in transient transfection experiments showed activity in both HSA expressing and non-expressing cell lines with a strength similar to that of the HSV TK promoter. Large fragments from the flanking region of the Cd24a promoter did not influence the ubiquitous nature of this promoter. Finally, the Cd24a, Cd24b and Cd24c genes were mapped to mouse chromosomes 10,8 and 14 respectively.
S15-03

POTENTIAL PHOSPHORYLATION SITES FOR PROTEIN KINASE A AND $C$ IN THE $\alpha$-SUBUNIT OF NA, $K$-ATPASE Beguin P., Beggah A.T. Rossier B.C.'Jaisser F. and Geering K. Institut de Pharmacologie et de Texicologie de inniversite, Ch-1005 lausanne Recent experimental evidence suggests that Na, KATPase mlght be a candidate for regulatory phosphorylation by protein kinases $A$ and $C$ (PKA and PKC). To verify this hypothesis, we have mutated several serine and threonine residues in consensus phosphorylation sequences of the $\alpha$ subunit of Bufo marinus Na, K-ATPase. The mutants were expressed in xenopus oocytes and phosphorylation was studied in oocyte homogenates upon stimulation of oocyte, PKA and PKC. Our results indicate that a unique phosphorylation site for PKA is located at serine 943 in the c-terminus of the $\alpha$-subunit but its phosphorylation can only be revealed in the presence of detergent. On the other hand. mutations of several serine and or threonine residues located ina serine and/or threonine phosphorylation did not or only partially abolish phosphorylation by PKC. In particular, mutations close to the catalytic phosphorylation site of the $\alpha$-subunit influenced phosphorylation by $\mathrm{RKC}$, suggesting that either this region contains a real phosphorylation site or is part of a conformational domain necessary for phosphorylation by PKC.

S15-04

\section{A SINGLE GENE ENCODES TWO ISOFORMS OF YEAST NADH-CYTOCHROME B5 REDUCTASE IN D I F F E R E N T SUBCOMPARTMENTS M I T O C H O N D R I A L}

Kerstin Hahne, Lynn Ramage and Gottfried Schatz

Biozentrum, Abt. Biochemie, Klingelbergstr. 70, 4056 Basel

We have cloned, sequenced and characterized the gene for the mitochondrial NADH-cytochrome b5 reductase $(M C R I)$ of Saccharomyces cerevisiae. Surprisingly, this gene encodes two mitochondrial isoforms of the flavoenzyme: a $34 \mathrm{kDa}$ integral protein exposed on the outer surface of outer mitochondrial membrane $\left(\mathrm{Mcrlp}^{34}\right)$, and a $32 \mathrm{kDa}$ soluble protein of the intermembrane space $\left(\mathrm{Mcrlp}^{32}\right)$. The smaller intermembrane space isoform is generated from the larger form by the action of the inner membrane protease I (IMPI) on the outer surface of the inner membrane.

This is the first demonstration that a single gene encodes proteins which are located in different compartments of the same organelle. 
S15-05

ATM1, A MITOCHONDRIAL ABC TRANSPORTER IN SACCHAROMYCES CEREVISIAE

$J$. Leighton and $G$. Schatz, Biocenter, University of Basel A PCR-based approach was used to identify new members in yeast of the superfamily of ATp-Binding Cassette (ABC) transporters, with special interest in any with a mitochondrial localization. Degenerate primers were designed on the basis of high homology between members of the ABC family, and PCR was performed on yeast genomic DNA. Ten DNA fragments bearing significant homology to the $A B C$ family were amplified, nine of which were previously unidentified.
Disruptions of five of the corresponding genes were performed. Disruption of one gene led to markedly impaired growth on rich medium and cessation of growth on minimal medium. The gene was cloned and found to encode a protein of 694 amino acids, a "half-transporter" which likely forms a dimer. Immunofluorescence on yeast expressing the gene cmyc-tagged at the c-terminus reveals co-jocalization of the protein with porin and with mitochoncrial DNA, visualized by DAPI staining. Nycodenz-purified mitochondria are enriched for the protein by immunoblotting. Additionally, the c-myc epitope is protease-protected in mitoplasts, indicating an inner membrane sub-localization and a probable orientation with the C-terminus in the matrix. The gene, termed ATM1 for ABC Transporter of Mitochondria, thus encodes the first member of the large $A B C$ transporter superfamily to be localized to this organelle. Present work is aimed at revealing the substrate of this transporter.

\section{S15-06}

PURIFICATION OF THE CARDIAC $\mathrm{Na}^{+} / \mathrm{Ca}^{2+}-\mathrm{EXCHANGER}$ EXPRESSED BY THE VACCINIA VIRUS SYSTEM WITH A 6xHIS-TAG

T. Iwata, D. Guerini and E. Carafoli, Laboratory of Biochemistry III, ETH zürich

The cardiac sarcolemmal $\mathrm{Na}^{+} / \mathrm{Ca}^{2+}$ exchanger is essential in the regulation of the contraction/relaxation cycle in cardiac myocyte. The molecular study of this protein has been difficult even after its over-expression was made possible by the cloning of the corresponding cDNA. The chief problem is the lack of convenient domains which could be used in the purification of the molecule. We have previously reported the efficient expression of the cardiac $\mathrm{Na}^{+} / \mathrm{Ca}^{2+}$ exchanger in mammalian cell lines using the T7 RNA polymerase-hybrid vaccinia virus system. We have now constructed the recombinant virus for the exchanger with a 6xHIS-tag at the c-terminal. This tag has enabled the purification of the protein through a Ni-NIA agarose column. The expressed tagged protein induced Na-dependent $\mathrm{Ca}$ uptake which was not different from that in intact cells, i.e., cells in which the exchanger did not have the tag. The most important aspects of the purification produced and those of the reconstitution of the expressed protein will be described.

\section{S15-07}

SUBCELIULAR TARGETING OF CHIMERIC ENDOPLASMIC RETICULUM/PLASMA MEMBRANE Ca $2+$ PUMPS

Davide Foletti, Danilo Guerini and Ernesto Carafoli, Institute of Biochemistry III, ETHzentrum, 8092 zürich

The sarcoplasmic/endoplasmic reticulum $\mathrm{Ca}^{2+}$-ATPase (SERCA) and the plasma membrane Ca ${ }^{2+}$-ATPase (PMCA) belong to the family of the P-type ion pumps. Whereas the SERCA is stricktly retained inside the cell, the PMCA is delivered to the plasma membrane. Since the two pumps are higly homologous, it was possible to construct PMCAchimeric proteins, carring $\mathrm{N}$-terminal sequences of the SERCA-ATPase, and express them in COS cells. Immunocytochemistry and other experiments have shown that a strong ER-retention signal is present in the first 150 aa of the SERCA (the region encompassing the first two transmembrane helices). Additional chimeras were then constructed, showing that both the first and the second transmembrane helix are involved in the ER-retention. Experiments with inverted chimeras (SERCA with the PMCA N-terminus) have indicated that in addition of the N-terminal region other sequences of the pump participate in the retention process.

$515-08$

PEST-SEQUENCES AND PROTEIN DEGRADATION

M. Molinari, E. M. Vilei, J. Anagli and E. Carafoli

Dept. of Biochemistry, ETH, Zurich

PEST-sequences are polypeptide regions rich in proline, glutamic acid, aspartic acid, serine and threonine (Rogers et al., (1986) Science $234,364-368)$. These sequences are found in proteins which are rapidly degraded by a nonubiquitin-mediated process catalyzed by calpain and/or several other intracellular proteases. The $\mathrm{Ca}^{2+}$-ATPase of erythrocytes, a preferred calpain substrate, contains 4 strong PEST-sequences surrounding the CaM-binding domain.

We have studied the correlation between the PEST-score and the susceptibility of the ATPase substrate to calpain digestion:

a) by deleting PEST-sequences in synthetic Cterminal portions of $\mathrm{Ca}^{2+-A T P a s e}$; b) by inserting mutations lowering the PEST-scores in the PESTsequences surrounding the CaM-binding domain of the $\mathrm{Ca}^{2+}$-ATPase expressed in E.Coli.

The experiments have shown that both the deletion of PEST-sequences and the lowering of the PEST-score make the $\mathrm{Ca}^{2+}-\mathrm{ATP}$ ase more resistent to calpain digestion.

\section{S15-09}

\section{A ROLE FOR THE NEURAL CELL ADHESION MOLECULE L1 IN THE STABILIZATION OF HIPPOCAMPAL LONG-TERM POTENTIATION}

A. Lüthi. M. Schachner* and J.P. Laurent. Pharma Division, Preclinical Research, F. Hoffmann-La Roche Ltd, CH-4002 Basel and Dept. of Neurobiology, Swiss Federal Institute of Technology, Hönggerberg, CH-8093 Zürich*

The neural cell adhesion molecule $L 1$ is involved in the formation and specification of cell contacts in the central and peripheral nervous system during development, and thus may also play a role in synaptic plasticity of the adult central nervous sysiem. Using extracellular recordings of evoked field potentials in the rat hippocampal slice, we found that locally applied polyclonal anti-L1 and fusion proteins containing the Ig-domains $|-V|$ of $L 1$ specifically block the stabilization of LTP in the CA1 region. Baseline synaptic transmission was not affected by the presence of the anti-L 1 antibodjes. The anti- $L T$-induced effect was dependent on the antibody concentration and anti$L 1$ antibodies had no effect on previously established LTP. LTP was also reduced by an antiserum against the recombinantly expressed Ig-domains I$V I$ of $L 1$, whereas control antibodies against liver cell membranes, which bind to neuronal membranes in the hippocampus, exhibited no effect on LTP.

The contribution of L1 to stabilization of LTP within the CA1 synapses suggests that members of the $\mathrm{lg}$-supertamily of cell adhesion molecules are involved in the structural modifications which are associated with synaptic plasticity in the mammalian central nervous system.

\section{S15-10}

\section{THE SAME C-TERMINAL REGION CAN SERVE AS A CONVENTIONAL PEPTIDIC SEGMENT OR INDUCE GPI- ANCHORING.}

C. Beghảadi-Rais, N.Déglon, C. Bron and N. Fasel, Institut de Biochimie, Université de Lausanne.

An increasing number of surface proteins are described as being attached to the membrane by a glycosyl-phosphatidylinositol (GPI) anchor instead of the conventional transembranous hydrophobic domain. They are initially synthesized with a $\mathrm{COOH}$ terminal polypeptide extension which is cleaved in the E.R. and replaced by the preformed glycolipid. This processing event is ditected by a signal, located in the C-terminal region of the protein, which requires a group of three small amino acids positioned $10-12$ residues upstream of an hydrophobic C-terminal sequence.

We previously transformed the transmembranous glycoprotein D of Herpes simplex type I into a GPI-anchored molecule by deleting its cytoplasmic domain and thus creating a molecule, gDww63, which meets the requirements for anchoring via a GPI structure. We now demonstrate, using GPI-deficient cell lines and biochemical studies, that a hybrid protein consisting of the Thy-1 ectoplasmic domain and of the C-terminal region of gDww63 can be alternatively expressed in a GPI-anchored or in a transmembranous form. 
S15-11

\section{PT RABBIT: A NOVEL POINT MUTATION IN THF PROTEOLIPID PROTEIN GENE}

Tosic,M., Dolivo,M., Domanska-Janik,K. and Matthieu,J.-M.

Service de Pédiatrie, CHUV, CH-1011 Lausanne

Proteolipid protein (PLP) is a major myelin protein in the central nervous system (CNS). A number of X-linked dysmyelinating disorders have been identified as mutations in the Plp gene. We have identified a novel Plp mutation in paralytic tremor $(P t)$ rabbit, characterized by body tremor, spastic limb paresis and hypomyelination in the CNS. Reduced levels of PLP protein and its mRNAs were observed in the CNS as well as in the PNS. Plp sequence analysis revealed a single nucleotide change in exon 2 which results in the substitution of a histidine by a glutamine at position 36. Histidine 36 is localized on the boundary between the first transmembrane region and the intracellular part of the protein. Therefore, its position can be crucial for the efficient PLP interaction with other proteins and lipids, and correct incorporation of the PLP molecule into the membrane. Histidine 36 is also part of a short fragment of ten amino acids which is conserved in all species studied. The same amino acid is altered in another hypomyelinated mutant, shaking pup, stressing its functional importance. Furthermore, the $P t$ mutation affects the PLP "premyelin" functions, including oligodendrocyte maturation.

\section{S15-12}

\section{THE N-TERMINAL HEPTAPEPTID OF MITOCHONDRIAI CREATINE KINASE IS IMPORTANT FOR OCTAMERIZATION}

Kaldis, Ph., Furter, R., Eppenberger, H.M, and Wallimann, T., Institute for Cell Biology, ETH-Hönggerberg, CH-8093 Zürich, Switzerland

Mitochondrial creatine kinase (Mi-CK) isoenzymes form octameric molecules composed of four stable dimers. The role of the charged amino acids within the N-terminal heptapeptide on the octamer stability was studied in detail by progressively substituting the four charged residues by uncharged ones. Equilibrium constants for octamer dissociation as well as dissociation rates in vitro increased in correlation to the number of charged residues eliminated, e.g. mutant 4-7, in which all four charged residues in the $\mathbb{N}$-terminal heptapeptide are substituted with uncharged amino acids, showed a 100-fold higher equilibrium constant for octamer dissociation and a 145-fold increased dissociation rate compared to wild type. This strongly suggests that the charged amino acids in the $\mathrm{N}$-terminal heptapeptide of $\mathrm{Mib}-\mathrm{CK}$, and therefore an ionic interaction, play an important role in forming the octameric molecule.

\section{S15-13}

GANGLIOSIDES AND NEUTRAL GLYCOSPHINGOLIPIDS ON HIV-POSITIVE PERIPHERAL BLOOD MONONUCLEAR CELLS. Leoni Lorenzo and Losa Gabriele A., Laboratorio di Patologia Cellulare, Istituto Cantonale di Patologia. Via in selva, CH-6604 Locarno

Gangliosides and neutral glycosphingolipids (GSLs) cell surface molecules are involved in a variety of biological processes and are assumed to play an important role in the mechanisms of HIV entry into lymphoid and epithelial cells. The total lipid-bound sialic acid (LBSA) content and the composition of gangliosides and GSLs were investigated on PBMN cells from HIV+ve and normal donors. LBSA level was quantified by a fluorimetric assay, while gangliosides and GSLS were assayed with high performance thin layer chromatography (HPTLC) after multistep lipid extraction of $100000 \mathrm{x} \mathrm{g}$ membrane fractions. In normal PBMN cells the LBSA content accounted for $2.6 \mu \mathrm{g} / 10^{8}$ cells or 20 $\mathrm{nmol} / \mathrm{mg}$ protein whilst in PBMN cells from HIV+ donors the LBSA level decreased to $1.6 \mu \mathrm{g} / 10^{8}$ cells or $13 \mathrm{nmol} / \mathrm{mg}$ protein. The quantitation of neutral GSLs and gangliosides was carried out by scanning spots revealed on HPTLC plates and the integrated areas computed with a dedicated software. Our findings showed that the most relevant ganglioside present in normal PBMNC was GM3 $(65-80 \%$ of total gangliosides) followed by small amounts of GD3 (5-15\%) and other acidic glycosphingolipids. Neutral GSLs included GL1, GL2, GL3 and GL4-hexosylceramide. HIV+ PBMN cells were characterized by a decrease of neutral glycosphingolipids as well as GM3 content which represented only $55 \%$ of the ganglioside fraction, indicating an alteration in the glycolipid composition in the plasma membrane of infected cells.
S15-14

IDENTIFICATION OF RESIDUES OF THE HUMAN NEUROPEP. TIDE Y RECEPTOR (Y1) IMPORTANT FOR LIGAND BINDING. Ph Walker, R. Martinez, M. Munoz, M. Sautel and M.C. Peitsch ${ }^{\star}$ Division of Hypertension, $\mathrm{CHUV}, \mathrm{CH}-1011$ Lausanne and * Institute of Biochemistry, UNIL, $\mathrm{CH}-1066$ Epalinges.

The neuropeptide $Y$ (NPY) is one of the most abundant peptides of the mammalian brain. Upon stimulation of cell surface receptors, NPY generates diverse physiologic responses. NPY acts on the cardiovascular system. It is a vasoconstrictor by itself and in addition, it potentiates the action of vasoactive substances such as angiotensin 11 or norepinephrine. NPY also inhibits glucose induced insulin secretion and is a potent inducer of appetife. To facilitate the development of NPY antagonists we are investigating the interaction of NPY with its cell surface receptor. We first constructed a series of mutants in which negatively charged residues present in putative extracellular domains of the $\mathrm{Y}_{1}$ receptor were systematically replaced by alanines. The mutant cDNAs were transiently expressed in HeLa cells using a vaccinia virus derived expression system and the ability of the encoded proteins to bind NPY was evaluated. The capacity of the mutant proteins to be recruited to the cell surface was assessed by confocal microscopy. We identified initially 4 spacially clustered extracellular acidic residues of the human Y1 NPY receptor essential for NPY binding. Subsequently, we mutated 36 additional residues. Based on these data a detailed computer model of the interactions between NPY and its receptor was built.

\section{S15-15}

GLUTAMATE UPTAKE BY ASTROCYTES: A MECHANISM LINKING FUNCTIONAL ACTIVITY TO ENERGY METABOLISM.

L. Pellerin and P.J. Magistretti. Institut de Physiologie, Université de Lausanne, Switzerland.

Astrocytes play a central role in brain energy metabolism. For example glucose, the exclusive brain energy substrate, is avidly taken up by astrocytes (PNAS 90:4042, 1993). Application of glutamate (Glu), the main excitatory neurotransmitter, stimulates in a concentration-dependent manner ${ }^{3} \mathrm{H}-2$-deoxyglucose (2-DG) uptake by astrocytes in primary culture with an EC50 of $\sim 100 \mu \mathrm{M}$. The effect is not receptor-mediated since it can not be reproduced by glutamatergic agonists such as (NMDA, kainate, quisqualate, $t$ ACPD) nor prevented by antagonists (APV, CNQX, AP3). Instead, it involves glutamate uptake since the effect is blocked by DL-threo- $\beta$ hydroxyaspartate, a potent inhibitor of the Glu transporter. Replacement of $\mathrm{Na}^{+}$in the medium by choline also prevents the effect, suggesting a $\mathrm{Na}^{+}$driven process. Finally ouabain, a selective inhibitor of the $\mathrm{Na}^{+} / \mathrm{K}^{+}$ATPase, completely prevented the effect of Glu. These observations suggest that when glutamatergic synapses are active, glutamate, taken up by astrocytes, stimulates 2-DG uptake by astrocytes whose end-feet are known to surround capillaries, i.e. the source of glucose. They also provide a simple explanation for the mechanism linking functional activity to energy metabolism.

\section{S15-16}

Cloning and molecular characterization of the mouse astrocyte glycogen synthase. G. Pellegri, C. Rossier, S. van Berchem, P.J. Magistretti and J.-L. Martin. Institut de Physiologie, Université de Lausanne, Switzerland.

In the brain glycogen is predominantly localized in astrocytes, although its presence has been detected in certain large neurons, in ependymal and choroid plexus cells. Glycogen is synthesized by the enzyme glycogen synthase (GlyS) from UDP-glucose. Out of the different glycogen synthase isozymes, only the muscle and the liver forms of GlyS have been cloned. We have isolated and characterized from a mouse astrocyte $\lambda$ ZapII cDNA library, a cDNA clone encoding the mouse cerebral cortical astrocyte GlyS. The $3.5 \mathrm{~kb}$ clone isolated contains an open reading frame of 2214 bp encoding a protein of 738 amino acids. The coding region of the mouse astrocyte GlyS cDNA shares $87 \%$ and $86 \%$ identity with the nucleotide sequences of the human muscle and the rabbit muscle GlyS cDNA respectively. The cellular distribution of the GlyS mRNA studied by Northern blot shows the presence of a single transcript of $4 \mathrm{~kb}$ in cultures of astrocytes and, to a lesser degree, of neurons. The distribution of glycogen phosphorylase, which was also examined by Northern blot shows the presence of a $4.2 \mathrm{~kb}$ transcript both in cultured astrocytes and neurons with however higher levels expressed in astrocytes. 
TARGET MOLECULAR SIZE OF THE VACUOLAR $\mathrm{H}^{+}$PYROPHOSPHATASE FROM MAIZE ROOTS

${ }^{*}$ Grünberger, S., \#Menger, A. and ${ }^{*}$ Chanson, A., ${ }^{*}$ Institute of Plant Biology and Physiology, University of Lausanne, Biology Building and \#Institute of Physical Chemistry, Swiss Federal Institute of Technology, CH-1015 Lausanne.

The functional molecular size of the vacuolar $\mathrm{H}^{+}$-pyrophosphatase $\left(\mathrm{H}^{+}\right.$PPase EC 3.6.1.1) from maize (Zea mays $\mathrm{L}$.) roots was estimated by radiation inactivation, both for substrate hydrolysis and for proton transport. Light microsomal vesicles enriched in tonoplast membranes were prepared from young (2 days) maize roots using sucrose step gradients. These vesicles were still competent for PPi-dependent proton transport after freeze-drying and rehydration of the membranes. Frozen native or freeze-dried samples were irradiated with ${ }^{60} \mathrm{Co}$ for various periods of time. After thawing the samples, the activities of glucose-6phosphate dehydrogenase (added as an internal standard) and $\mathrm{H}^{+}$-PPase (hydrolysis of pyrophosphate (PPi) and PPi-dependent proton transport) were tested. By applying target theory, the functional size for hydrolysis was found to be around $M_{r} 155000$ when the native or freeze-dried samples were irradiated. No PPi-dependent proton transport activity was measurable after irradiation of the native samples. On the contrary, the freeze-dried membranes were still pumping protons after irradiation and a target size of around $\mathrm{M}_{\mathrm{r}} 250000$ was measured for the transport activity. These experiments suggest that the native $\mathrm{H}^{+}$-PPase from maize roots may exist as a dimer (at least) of the $\mathrm{M}_{\mathrm{r}} 80800$ catalytic subunit.

\section{S15-18}

SODIUM TRANSLOCATION BY THE Na,K-PUMP: ROLE OF THE NTERMINUS OF THE a SUBUNIT. Wang, $X$. and forisberger $J .-D$. Institut de Pharmacologie et de Toxicologie de l'Université, Lausanne.

The $\mathrm{Na}, \mathrm{K}$-pump noves $3 \mathrm{Na}^{+}$out and $2 \mathrm{~K}^{+}$ions into the cells. The domains of the protein involved in ion translocation have not been determined. We have studied the role of the $\mathrm{N}$-terminal region in the $\mathrm{Na}^{+}$translocation by measuring the kinetics of charge movements under $\mathrm{Na} / \mathrm{Na}$ exchange conditions in wild type (WT) and two N-terminally truncated forms of the Bufo a subunit with deletions of the $31\left(\mathrm{~T}_{31}\right)$ and $40\left(\mathrm{~T}_{40}\right)$ first amino acids expressed in Xenopus oocytes. We have developped a new technique to perform fast ( $<1$ ms) voltage clamp on whole oocyte. The membrane on one side of the cocyte is permeabilized by digitonin allowing to measure current flowing across the other half. We observed presteady state ouabain-sensitive currents similar to that reported by other techniques (J.Gen.Physiol. 101:117,1993). The estimated forward charge translocation rate was lower in both $\mathrm{N}$-truncated mutants (WT $430 \pm 14, \mathrm{~T}_{32} 215 \pm 8, \mathrm{~T}_{41} 124 \pm 3 \mathrm{~s}^{-1}$ ). The midpoint potential of the charge translocation (best fit to Boltzman equation) was $-79,-45$, and $-28 \mathrm{mV}$ in the WT, $\mathrm{T}_{31}$ and $\mathrm{T}_{40}$ groups, respectively. The highly charged $\mathrm{N}-$ terminus of the a subunit has a significant role in the forward translocation of $\mathrm{Na}^{+}$ions.

INTERNALIZATION OF INFLUENZA VIRUS HAY-543 MUTANTS SUGGEST A MODEL FOR INTERNALIZATION SIGNALS CONTAINING TYROSINE. Hussein Y. Naim, and Michael G. Roth; Dept of Biochemistry, UT-Southwestern Medical center. at Dallas, Dallas, Texas-USA We have investigated an artificial internalization signal created by site-directed mutagenesis of the short cytoplasmic sequence of the influenza virus hemagglutinin $(\mathrm{HA})$. Mutation of cysteine 543 to tyrosine converted $\mathrm{HA}$ from a protein essentially excluded from coated pits to one that internalized at rate similar to some cellular endocytic receptors. To determine whether or not the HA-Y543 signal is a degenerate form of the internalization signal found in proteins such as the transferrin receptor and the mannose-6-phosphate/IGFII receptor, we have mutated amino acid positions in the HA-Y543 shown to be important for internalization of the two receptors. We have changed amino acids on either side of $Y 543$ to those that either fit, or break the pattern of aromatic and hydrophobic residues proposed as consensus sequence. Our results indicate that the HA-Y543 mutant contains a sub-optimum sequence for a tyrosine-based internalization signal similar to those found in the receptors for transferrin, LDL, and mannose-6-phosphate/IGFII. However, amino acids with side chains having different chemical properties functioned well in positions that are important for the internalization signal, indicating that the consensus sequence for this signal is more variable than previously proposed.
TIGHT JUNCTIONAL PARTICLES AND PERMEABILITY FOR PEROXIDASE IN NORMAL AND CHOLESTATIC RAT LIVER

C. Rahner and L. Landmann, Dept. of Anatorny, University of Base1, $\mathrm{CH}-4056$ Basel.

In order to correlate tight junctional (TJ) structure with paracellular permeability in the in situ liver, normal and cholestatic rat hepatocytes were compared. Cholestasis was induced by bile duct ligation for $48 \mathrm{~h}$ (BDL), or ethinylestradiol $(5 \mathrm{mg} / 100 \mathrm{~g} \mathrm{bw})$ for $5 \mathrm{~d}(\mathrm{EE})$. Size and spacing of $\mathrm{TJ}$ particles, indicating the quality of the TJ strands, were assessed in replicas of high pressure frozen, chemically unfixed liver and showed no statistically significant differences between the groups. Permeation of horseradish peroxidase (HRP) through the hepatocyte tight junctions was estimated cytochemically using exogenous HRP as a tracer. Almost no TJ were penetrated by HRP in controls, while $50 \%$ and $15 \%$ were HRP positive in the BDL and EE groups, respectively. We conclude from these and our earlier data (Experientia 49: A47, 1993) that cholestasis does not affect TJ organization at the level of singular strands but that overall functional integrity is impaired by BDL more than by EE.
S15-21

\section{PROTEIN DOMAINS IN LACTASE-PHLORIZIN HYDROLASE (LPH) IMPLICATED IN CELLULAR TRANSPORT TO THE PLASMA MEMBRANE}

Petra Panzer and Hassan Y. Naim, Institut für Mikrobiologie, Heinrich-Heine-Universität Düsseldorf, Universitätsstr. 1, D-40225 Düsseldorf, Germany

Deletion mutants of human small intestinal lactase-phlorizin hydrolase (LPH) were constructed to determine the role of protein subdomains in the intracellular transport of LPH. The mutant LPH1646MACT (236 aa deletions), which contains the membrane anchor (MA) and the cytoplasmic tail (CT), was transported to the cell surface in a fashion similar to wild type LPH. By contrast, LPH1646 lacking the transmembrane domain persisted as a mannose-rich polypeptide. This suggests that the membrane anchor and/or cytoplasmic tail are implicated in the ER-egress of LPH. Another mutant, LPH1559MACT (323 aa deletions), was not efficiently transported to the Golgi apparatus. Epitope mapping with monoclonal antibodies as well as protease-sensitivity assays provided an evidence that the tertiary structure of LPH 1646 MACT is very similar to that LPH1559MACT. In view of the variations in the proportions of the complex glycosylated molecules of the two mutants we conclude that the domain between LPH1646MACT and LPH1559MACT may play an essential role along the secretory pathway of LPH.

S15-22

\section{THE DISULFIDE-BONDING PATTERN OF HUMAN COMPLEMENT COMPONENT C9}

S.Lengweiler, D. Wymann, J. Schaller and E.E. Rickli, Institute of Biochemistry, University of Berne, $\mathrm{CH}-3012$ Bern

$\mathrm{C9}$ is the last acting protein in the complement cascade. Binding of $\mathrm{C} 9$ to a membrane bound precursor complex composed of proteins $\mathrm{C} 5 \mathrm{~b}, \mathrm{C} 6, \mathrm{C} 7$ and $\mathrm{C} 8$ completes the formation of the cytolytic membrane attack complex.

Mature C9 is a single chain glycoprotein, comprising 537 residues. The 24 half-cystines are all linked to disulfide bonds. Most of them occur in positions well conserved across the terminal complement components.

$\mathrm{C} 9$ was isolated by fractionation with $\mathrm{BaCl}_{2}$ and polyethylene glycol followed by chromatography on lysine-Biogel, DEAESephacel and hydroxylapatite. To determine the disulfide bonds $\mathrm{C} 9$ was cleaved with various enzymes and reagents. The digest generated was separated by RP-HPLC. Cystine containing peptides were detected by means of amino acid analysis and fluorescence labelling. Final identification of the disulfide bonds was obtained by Edman degradation. 


\section{S15-23}

MOLECIJLAR CLONING AND CHARACTERIZATION OF ALG GENES

OF SACCHAROMYCES CEREVISIAE

Stagljar I., te Heesen S., Zufferey R., Wyder L., Aebi M., Institut für Molekularbiologie I, Universität Zürich, Hönggerberg, 8093 Zürich, Switzerland.

$\mathrm{N}$-linked glycosylation is an essential protein modification occuring in all eukaryotic cells. Core-oligosaccharides are transferred by the enzyme N-oligosaccharyl-transferase from the donor Glc Man GlcNAC -Dolichol to selected asparagine residues of the nascent polypeptide chain. The donor is build up in a stepwise fashion at the ER-membrane by the products of the ALG-genes (asparagine-Iinked-glycosylation) Mutants defective in this pathway are available, but often lack a phenotype suitable for isolation of the corresponding genes. We found that double mutants of alg5 or alg8 with a wbpl mutation (defective oligosacchary $\overline{l-t r a n s} \overline{\text { ferase }}$ ) are severely growth retarded at $30^{\circ} \mathrm{C}$. The double mutant phenotype allowed the isolation of both ALG5 and ALG8 genes which have been refractory to cloning so far. Both genes encode potential transmenbrane proteins and are able to cormplement the biochemical defects of the corresponding alg5 or alg8 mutants.

\section{S15-24}

EXPRESSION OF AN OUABAIN-RESISTANT Na,K-ATPase $\alpha$-SUBUNIT IN XENOPUS LAEVIS A6 KIDNEY CELLS

Beron, J. and Verrey, F., Physiologisches Institut der Universität Zürich, CH-8057 Zürich

The Na,K-ATPase produces the driving force for the regulated transport of $\mathrm{Na}$ across epithelial cells. It is composed of an $\alpha$-subunit which carries the catalytic and ouabain binding sites and a B-subunit. To test the role of the $\alpha$ subunit in transport regulation, we transfected A6 cells with the $B$. marinus $\alpha 1$-subunit $(\alpha 1$-TBM). This subunit confers a 300 -fold higher resistance to ouabain $\left(\mathrm{K}_{\mathrm{i}} \approx 50 \mu \mathrm{M}\right)$ compared to the endogenous pump. Taking advantage of this difference, stable transfectants were selected with ouabain. Expression of the $\alpha 1$-TBM subunit was visualized by Western blotting. Approximately half of the positive cell lines showed a high electrical resistance similar to untransfected cells, when cultured on filters. Na-transport activity, measured as $I_{S C}$, was lower even in the absence of ouabain, but the relative increase in response to aldosterone was maintained. Functional pumps containing the exogenous or endogenous $\alpha 1$-subunit were quantified by equilibrium ouabain binding. Interestingly, cells cultured on plastic dishes had a large fraction of low affinity binding sites ( $\alpha 1-$ TBM), while most sites were of the high affinity type when the cells were grown on filters. We conclude that the ouabain-resistant $\mathrm{Na}, \mathrm{K}$-ATPase $\alpha 1$-subunit of $B$. marinus can be expressed in A6 cells and used as a selective marker. However, the results suggest that the expression of functional ouabain-resistant pumps containing the $\alpha 1$-TBM subunit could be influenced by the degree of cellular differentiation.

\section{S15-25}

FUNCTIONAL DOMAINS IN THE CYTOSKELETAL PROTEIN TALIN V. Nigg1i (1), S. Kaufmann (2), W.H, Goldmann (2), G. Isenberg (2), (1) Dept. of Pathology, University of Bern, CH3010 Bern and (2) Biophysics Department, Technical University of Munich, D-85747 Garching, Germany

Talin interacts in vitro with actin, vinculin, integrins and bilayers of acidic phospholipids, and nucleates actin filament growth at the bilayer surface. It is therefore believed to be a key protein mediating actin-membrane linkage. We have analyzed functional properties of proteolytic fragments of purified platelet talin. Using limited proteolysis two fragments of 47 and $190 \mathrm{kD}$ were generated. Preliminary results, obtained using viscometry and F-actin-nucleating assays, suggest that the $190 \mathrm{kD}$ fragment contains the actin binding site. We also assessed the lipid-binding capacity of the fragments. When a mixture of the two fragments was centrifuged in the presence of large multilamellar liposomes containing phosphatidylserine, only the $47 \mathrm{kD}$ fragment, but not the $190 \mathrm{kD}$ domain, co-sedimented with the liposomes, suggesting the presence of a specific lipid-binding site in the $47 \mathrm{kD}$ domain. Interestingly, this fragment contains the $\mathbb{N}$-terminus of talin which shows homologies to the membrane-binding regions of protein 4.1 . We are now analyzing membrane-binding properties of talin domains in more detail using hydrophobic photolabelling.
S15-26

\section{A heparin binding site on the 5th FN-III domain of chicken tenascin}

Weber, . , Zimmermann,D.R., winterhalter,K.H. and Vaughan, I.

Glypican, a GPI anchored membrane protein, binds to tenascin by its heparan sulfate chains. To identify potential heparin binding sites on tenascin, we prepared a series of fusion proteins derived from the fibronectin type III homology region. Affinity chromatography on heparinSepharose of these fusion proteins indicates that a new heparin binding site may exist on domain 5 . This was confirmed using solid phase assays with labelled heparin. The heparin binding is specific and can not be inhibited by chondroitin sulfate or dermatan sulfate. This binding site may modulate cell-matrix interactions between cell surface proteoglycans and tenascin.

\section{S15-27}

FUNCTIONAL EXPRESSION OF A RENAL Na/P. COTRANSPORTER (NaPi-2) USING THE BACULOVIRUS EXPRESSION SYSTEM.

Fucentese M., Murer H. and Biber $J$. institute of Physiology, University Zürich-Irchel, 8057 Zürich

The $\mathrm{Na} / \mathrm{P}$-cotransporter $\mathrm{NaPi}-2$ is a protein expressed in the brush-border membrane of proximat tubular cells of the rat kidney. Sf9 cells, derived from Spodoptera frugiperda, were infected with a recombinant baculovirus and analyzed for expression of the $\mathrm{NaPi}-2$ protein. In infected cells fully functional $\mathrm{Na} / \mathrm{P}_{\mathrm{i}}$-cotransport was found in a time dependent manner: up to a 50-fold stimulation of $\mathrm{Na} / \mathrm{P}_{-}$-cotransport compared to uninfected cells was observed after 3-4 days. Transport of phosphate by infected cells was highly dependent on the presence of sodium, exhibited a $\mathrm{Km}$, around $0.16 \mathrm{mM}$ and was dependent on extracellular $\mathrm{pH}$ ( ) in agreement with expressed transport of phosphate, infected cells produced an immunoreactive polypeptide of $66 \mathrm{kDa}$ that represents a non-glycosylated form of the 80 to $90 \mathrm{kDa}$ mature $\mathrm{Na} / \mathrm{P}_{\text {-cotransporter. }}$-cot

We conclude that the protein NaPi-2/rat when expressed in St9 cells exhibits $\mathrm{Na} / \mathrm{P}_{\text {- }}$-cotransport with similar kinetic characteristics as observed in proximal tubular apical $\mathrm{Na} / \mathrm{P}_{\mathrm{i}}$ cotransport. Therefore the NaPi-2 protein as expressed ih Sf9 cells can be used for further structural and functional studies.

\section{S15-28}

Role of the Neural Cell Adhesion Molecules L1 and NCAM in the Performance of a Spatial Navigation Task in Wistar Rats.

Arami S., Greipl R., * Jucker M., Welzl H., ${ }^{*}$ Schachner M., Lab. Behavioral Biology and Lab. Neurobiology, ETHZürich, CH-8092 Zürich

Recent studies suggest a role of the neural cell adhesion molecules L1 and NCAM in mechanisms of memory formation (Doyle $E$. et al, $J$. Neurochem. 59:1570-1573, 1992; Scholey A.B. et al, Neuroscience 55:499-509, 1993; Lüthi A. et al. unpublished data and see USGEB 94). in the present study we analyzed the effect of chronic intraventricular infusion of polyclonal antibodies against L1 (anti-L1) or NCAM (antiNCAM) on the performance of male Wistar rats during the acquisition and retention of a spatial learning task. Briefly, rats had to remember the position of a submerged escape platform in a water tank (Morris water-maze). When the escape platform was removed after acquisition training ( $=$ retention test), the performance of animals chronically injected with anti-L1 showed an impaired search pattern when compared with that of saline-injected animais ( $p>0.05$, Mann-Whitney). compared with that of saline-injected animais ( $p>0.05$, Mann-Whitney).
Whereas control animals spent up to $45 \%$ of their time searching for the platform in the correct ( = training) quadrant, the time anti- $L 1$ animals swam in the correct quadrant was closer to chance level $(31 \%)$ Although monitoring penetration of anti-NCAM into the hippocampus was almost as efficient as that of anti- $L 1$, the performance of the antiNCAM-group was highly variable and did not differ from the performance of controls. These results agree with recent findings on the involvernent of neural cell adhesion molecules in other forms of learning. 
S15-29

FUNCTIONAL EXPRESSION OF THE $\alpha$-SUBUNIT OF THE GASTRIC H, K-ATPASE OF MOUSE AND XENOPUS

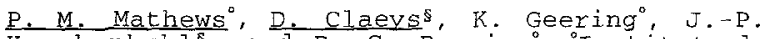
Kraehenbuhls, and B. C. Rossier'; 'Institut de Pharmacologié et de Toxicologie, Université de Lausanne; and Institut suisse de Recherche Expéximental sur le cancer et Institut de Biochimie, Université de Lausanne

We have isolated CDNAs from mouse and from Xenopus encoding the $\alpha$-subunit of the gastric $H, K-A T P a s e$, which is expressed in the parietal cells of the stomach mucosa. The gastric H, KATPase transports $\mathrm{H}^{+}$into the stomach Iumen with the counter transport of $\mathrm{K}^{+}$, all at the expense of ATP hydrolysis. When Xenopus oocytes are injected with cRNA encoding $\alpha_{H}, K$ from either species, as well as a gastric $\beta_{H}, K$ subunit, the two subunit proteins are synthesized, assemble, and form functional pumps located in the plasma membrane as demonstrated by ${ }^{86} \mathrm{Rb}^{+}$uptake. The sensitivity to the gastric H,K-ATPase inhibitor SCH28080 was determined, with the $\mathrm{K}_{\mathrm{i}}$ Eor both species found to be in the low $\mu \mathrm{M}$ range.

S15-30

CLONING AND FUNCTIONAL ANALYSIS OF THE $\beta$-SUBUNIT OF THE XENOPUS GASTRIC H, K-ATPASE

P. $-x$. Chen, E. M. Mathews, K. Geering, and B. C. Rossier; Institut de Pharmacologie et de Toxicologie, Université de Lausanne

We have isolated from a Xenopus stomach CDNA library a $1.1 \mathrm{~kb}$ clone encoding the $\beta$-subunit of the gastric H,K-ATPAse. The deduced protein sequence consists of 295 amino acids with about 55\% amino acid sequence identity when compared to

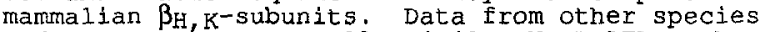
and from the structuraliy similar Na,K-ATPase has shown that the $\beta$-subunit is necessary for the formation of a functional pump, which consists of an $\alpha / \beta$ heterodimer. Following co-injection of Xenopus oocytes with cRNA for both the $\alpha$ - and $\beta$ subunits, we have observed by $\mathrm{Rb}^{+}$uptake $\mathrm{H}, \mathrm{K}-$ ATPase activity in the plasma membrane. We are presently studying the role of the $\beta$-subunit in the maturation and function of the H,K-ATPase.

$515-31$

EXPRESSION OF H,K-ATPASE IN XENOPUS OOCYTES TO STUDY ITS ROLE IN AUTOIMMUNE GASTRITIS

D. Claevs ${ }^{\circ}$, P. M. Mathews\$, K. Geexings, B. C. Rossiers, and J.-P. Kraehenbuhl"; "Institut Suisse de Recherche Expérimental sur le Cancer et Institut de Biochimie, Université de Lausanne: and ${ }^{8}$ Institut de Pharmacologie et de Toxicologie, Université de Lausanne

Auto-immune gastritis (AIG) is accompanied by auto-antibodies to the H,K-ATPase of the gastric parietal cell. Neonatal thymectomy of BALB/C mice induces AIG. We have cloned the $\alpha$ - and $\beta$ subunits of the BALB/C H,K-ATPase and expressed subunits of the BALB cRNA injected aocytes. Both $\alpha$ the proton pump in cRNA injected oocytes. Both pumps, and are immunoprecipated using auto-immune sera. The $\boldsymbol{\beta}$-subunit can also be immunoprecipated independent of the $\alpha$-subunit when oocytes are injected with $\beta$ CRNA alone. BALB/c mice will be immunized with oocyte membranes containing either the $\alpha / \beta$ or $\beta$ antigens in order to study the role of each $\mathrm{H}, \mathrm{K}$-ATPase subunit in triggering $\mathrm{AIG}$
S15-32

Atomic force microscope study of Spodoptera Frugiperda cell membranes

R. Cargnello, J.M. Solletti, S. Kasas

Institute of Histology and General Embryology, University of Fribourg, Faculty of Sciences, CH-1700 Fribourg, Switzerland

Spodoptera Frugiperda cells are often used to overexpress eucaryotic proteins using Baculovirus vectors.

The atomic force microscope (AFM) is a device which allows the observer to obtain high resolution images of biological samples immersed in a fluid medium; this instrument has therefore the potential to visualize overexpressed proteins on the surface of living cells.

However, the interpretation of the images is sometimes difficult and requires a good knowledge of the specimen topography, i.e. the "normal" plasma membrane of the cell used for the overexpression. On our poster we present AFM images of the plasma membrane of both fixed and living Spodoptera Frugiperda cells.

In additon, we discuss the possibility of achieving high resolution in the imaging of dynamic changes which affect living systems.

S15-33

Measuring the tip interaction with biological samples

Jean-Marie Solletti, Albrecht L. Weisenhorn, Raffaella Cargnello and Sandor Kasas

Institute of Histology, University, Pérolles CH1700 Fribourg, Switzerland

The atomic force microscope (AFM) is a new type of microscope which allows visualisation of inorganic and organic samples with a very high resolution.

A sharp tip fixed at the end of a cantilever is used as a topographic sensor. By scanning the sample with this device, a computer records the minute deformations of the cantilever and computes the topograplyy of the sample. The instrument allows also the measurement of the interaction between the tip and the sample as a function of the distance.

The knowledge of these interactions allows us to compute several mechanical properties like indentation, elasticity and stiffness.

On this poster we report the results of these measurements on cells (Sf9, CI31) bacteria (E. coli, B. subtilis) and proteins (actin).

In addition we discuss the use of the AFM as a sensor for probing the mechanical properties of biological systems at a nanometric scale.

\section{S15-34}

The prion protein is expressed in both astrocytes and oligodendrocytes of the neonatal brain and is down-regulated in adulthood.

M. Moser, Colello, R., Pott, U. and B. Oesch

Institut für Hirnforschung der Universität Zürich, August Forel Str. 1, 8029 Zürich

The infectious agent causing transmissible spongiform encephalopathies consists largely or entirely of PrPSc, a modified isolorm of normal PrP (PrPC) PrPc is most abundant in the brain. The development of the disease strictly depends on the presence of $\mathrm{PrPC}$, and incubation times are shortened by overexpression of PrPC. The normal function of PrPC is not known and its cellular localisation in the CNS is poorly characterised, except for its evident presence in neurons. Here we describe the expression of PrPC mRNA in both oligodendrocytes and astrocytes during neonatal development of hamster and rat. In adult animals, expression is down-regulated in glia but not in neurons. Our findings provide a link to the previously described accumulation of PrPSC in astrocytes and the apparently faster course of prion disease in neonatal hamster. Our results argue for a major involvement of non-neuronal cell types in prion diseases. 
IDENTIFICATION AND CHARACTERIZATION OF A CELLSURFACE PROTEIN POSSIBLY INVOLVED IN METASTASIS Bachmann, F. and Burger, M.M., Friedrich Miescher-Institut. P.O. Box 2543 CH-4002 Basel

Extracellular matrix and cytoskeleton elements are dynamically linked by membrane structures allowing transduction of various kinds of signals. Metastatic cells. compared to normal cells, often show altered composition of signaling complexes which frequently result in changes in cell movement, adhesion properties and morphology. In order to investigate such structures we raised Mab against membrane protein fractions. A Mab against a vitronectin receptor containing fraction was found, which if applied in vitro to $B 16 \mathrm{~F} 1$ mouse melanoma cells, influenced growth, adhesion and morphology. When B16F1 cells prior to tail vein injection were treat vein injection were tre observed. Short treatment ( 1 h) resulted in $85 \%$ inhibition of lung colonization whereas long-term tre

a candidate molecule likely involved in these observed A candidate molecule likely involved in these observed protein. Seguence information from peptides revealed in several cases $100 \%$ homology to mouse centrosomin $A$, i.e.a $32 \mathrm{kDa}$ cytosolic protein involved in the organization of the spindle apparatus. These data suggest that we may have found a protein which may participate in a link betwern the extra-cellular space and the microtubular system.

We are currently trying to clone this $150 \mathrm{kDa}$ protein from a B16F1 C-DNA library.

\section{S15-36}

DETERMINANTS FOR MISSORTING OF A MUTANT OF THE ASGP-RECEPTOR SUBUNIT HI

Wahlberg, J.M., and Spiess, M.

Dept.Biochemistry, Biozentrum, University of Basel. Signals for correct basolateral sorting of subunit H1 of the human asialoglycoprotein receptor are located in the cytoplasmic tail, involving a tyrosine at position 5. Removal of this residue results in nonpolarized transport of the protein to the surface in MDCKII-cells. A deletion mutant of $\mathrm{Hl}$, lacking 30 of the normal 40 residues of the cytoplasmic tail, including the tyrosine, has been found not to be transported to the cell surface, but to be accumulated intracellularly. The mutant protein is complex glycosylated and sialylated, indicative of passage into or through the transGolgi. Immunofluorescence analyses show defined juxtanuclear, tubular structures, which do not colocalize with markers for ER, early endosomes or lysosomes, but which show similar staining pattern as markers for late endosomes and TGN. To define the determinants responsible for the intracellular accumulation a series of deletions and fusions have been constructed. The results suggest that the length of the cytoplasmic domain is one important determinant for missorting of $\mathrm{H} 1$.

\section{S15-37}

\section{E-CADHERIN (UVOMORULIN) : THE CAICIUM DEPENDENCE OF THE CONFORMATION}

Pokutta, S.; Herrenknecht, K.; Engel, J.; Abt. Biophysikalische Chemie, Biozentrum, $\mathrm{CH}-4056$ Basel,; MPI für Imunbiologie, D-7800 Freiburg

E-cadherin belongs to the class of Ca2t-dependent cell adhesion molecules. To investigate the $\mathrm{Ca}^{2+}-$ binding properties of the protein we expressed the extraceliular part, consisting of 4 homologous domains, using the baculovirus expression vector system. It has been shown that upon $\mathrm{Ca}^{2+}$ removal the molecule undercoes a conformational change. This change is already visible in the electron microscope. In ultra-centrifugation experiments different sedimentation coefficients in presence $\left(s_{20 w}=3.5 S\right)$ and in absence of $\mathrm{Ca}^{2+}\left(s_{20 w}=3.0 S\right)$ are observed. The calcium dependent structural changes could also be monitored by fluorescence spectroscopy and circular dichroism. By titrating the $C D$ signal a mean $K_{d}$ of $52 \mu M$ was obtained. By removal of $\mathrm{Ca}^{2+}$ the trypsin cleavage rates of the molecule increases $\left(\mathrm{K}_{\mathrm{d}}=22 \mu \mathrm{M}\right)$. The main trypsin cleavage site lies between the two putative Cat+ binding sites of domain III.
CHARACTERIZATION OF A NEW $\beta_{3}$-ADRENERGIC RECEPTOR RADIOLIGAND: [ $\left.{ }^{3} \mathrm{H}\right] \mathrm{SB} 206606$

P.Muzzin, O.Boss, N.Mathis, J.P.Revelli,

J.P.Giacobino K.Willcocks, G.T.Badman,

B.C.C.Cantello, R.M.Hindley and M.A. Cawthorne Bioméd., CMU, 1 M. Servet, Geneve and SmithKline Beecham Pharmaceuticals, Surrey, U.K.

The $\beta_{3}$-adrenergic receptor (AR), recently cloned from a rat CDNA library, was found to be expressed in rodent adipose tissue. Until now, classical $\beta$-adrenergic radioligands, [ ${ }^{3} \mathrm{H}$ ]CGP 12177 and [125I]ICYP, have been used to characterize the $\beta$-ARs in fat cell membranes, where the $\beta_{1}-, \beta_{2}-$ and $\beta_{3}-A R$ subtypes coexist, but these radioligands have a very low affinity for the $\beta_{3}-A R$. It was thus of interest to obtain a radioligand which would bind selectively to the $\beta_{3}-A R$. For this purpose the RR-enantiomer of the $\beta_{3}-A R$ agonist $B R L$ 37344 was tritiated. This new radioligand, called $\left[{ }^{3} \mathrm{H}\right] \mathrm{SB} 206606$, was characterized by binding studies using membranes of both $\beta_{3}-$ AR transfected CHO-KI cells and rat brown adipose tissue. The similarity of the results obtained in both systems indicates that $\left[{ }^{3} \mathrm{H}\right] \mathrm{SB} 206606$ binds selectively to the $\beta_{3}-A R$.

\section{S15-39}

BINDING OF FIBRINOGEN AND CYCIIC RGD-PEPTIDES TO $\alpha I I b \beta 3$ RECONSTITUTED INTO PLANAR LIPID BILAYERS

Tangemann, K., Müller, B., Engel, J., Department of Biophysical Chemistry, Biocenter Basel

The olatelet integrin $\alpha I I b \beta 3$ was isolated and reconstituted into lipid vesicles. As judged by electron microscopy the integrin incorporated adequately only into 1,2-dimyristoylglycero-3phosphocholine/1,2-dimystoylphosphatidylglycerol vesicles after removal of the detergent by adsorption to Bio-beads. These vesicles were then used to generate planar lipid bilayers. The binding of rhodamine labelled fibrinogen, the peptide GRGDSFC, and several cyclic penta- and hexa-RGD-peptides was monitored by total internal reflection fluorescence microscopy (TIREM). Analysis of the kinetics revealed a two step binding mechanism. Fast reversible formation of a fibrinogen-integrin precomplex $\left(\mathrm{K}_{\mathrm{D}}=50 \mathrm{nM}\right)$ is followed by formation of a stable irreversible complex as judged by the lack of dissociation after addition of excess GRGDS. For the peptides the rates of association and dissociation were faster than the time resolution of the method. The RGD-peptides showed about 100-fold lower binding affinities than fibrinogen.

\section{$515-40$}

\section{EXPRESSION AND SUBCELLULAR DISTRIBUTION OF CHOLINE ACETYLTRANSTERASE IN TRANSFECTED CELLS}

Salem, N., Medilanski, J., Pellegrinelli, $N$. and Eder-Colli, L Departement of Pharmacology, CMU, 1211 Geneva 4

In cholinergic neurons the enzyme choline acetyltransferase (ChAT) exists as cytosolic(80-90\%) and membrane-bound activity $(10-20 \%)$ Are these two activities encoded by a single mRNA? Is membranebound enzyme preferentially associated with a given cellular membrane? In order to investigate these questions we isolated a fulllength cDNA (4.2 kb) encoding Drosophila ChAT and used it to transfect Xenopus oocytes, rat fibroblasts and human neuroblastoma cell lines. Triton X-114 fractionation of transfected cells showed that hydrophilic and amphiphilic ChAT activity were produced. The sp act. of hydrophilic enzyme reached $2,0.8$ and $0.25 \mu \mathrm{mol} \mathrm{ACh} / \mathrm{h} / \mathrm{mng}$ protein respectively in oocytes, fibroblasts and neuroblastoma whereas the sp. act. of amphiphilic enzyme was $1.8,0.4$ and $0.125 \mu \mathrm{mol} \mathrm{ACh} / \mathrm{h} / \mathrm{mg}$ protein, respectively. Amphiphilic ChAT was less sensitive to inhibition by the product acetylcholine and to heat denaturation than was hydrophilic enzyme. Similar results were observed for the native Drosophila ChAT. Amphiphilic enzyme sedimentation was affected by the type of detergent present in linear density gradients of sucrose. Transfected oocytes were subjected to subfractionation. Besides a large amount of soluble ChAT activity, a significant proportion of enzyme was found associated with a fraction enriched in endoplasmic reticulum (ER). Preliminary results using transfected mammalian cells indicate that apart cytosolic ChAT, plasma membranes+Golgi enniched fractions as well as ER-enriched fraction contain ChAT activity. 
S15-41

TESTIS EXPRESSION OF A PROTEIN BELONGING TO THE NEUROTRANSMITTER TRANSPORTER FAMILY

D. Bigler, R.E. Dörig, S. Schwendener, B. Suter, and E. KubIi, Zoological Institute, University of Zürich-Irchel, winterthurerstrasse 190, 8057 zixich, Switzerland.

We have characterized a Drosophila melanogaster gene encoding a very hydrophobic protein. The amino acid sequence shows considerable homology (33-42\% identity) to neurotransmitter transporters. The gene is, however, not expressed in neural tissue, but in the male germine. Transcription and translation occurs in early spermatocytes. Antibody staining data suggest the following pathway of the protein during spermatogenesis: The protein is synthesized in early spermatocytes and is transported into the nucleus during prophase of RT 1. At meiosis it associates with the mitochondria and it stays associated while they fuse to the Nebenkern. During individualization it is stripped off in the cystic bulge. Inspection of the putative protein sequence shows that it possesses alle the necessary targeting signals to allow its transfer from the cytoplasm through the nucleus into the mitochondria: three nuclear targeting signals, a mitochondrial import signal and, moreover, two PEST-sequences for rapid protein degradation are present.

We shall present a, currently tested, working hypothesis.

\section{S15-42}

COPPER DECREASES THE STATE OF HIGH WATER

PERMEABILITY IN Hg-TREATED TOAD SKINS.

Grosso A. \& de Sousa R.C., Dept of Physiology, C.M.U., Faculty of Medecine, 1211 Geneva 4, Switzerland

In $\mathrm{Hg}$-treated toad skins, water permeability is high or low depending on whether the major anion of the inner bathing medium is $\mathrm{SO}_{4}$ or $\mathrm{Cl}$. We report here the results of experiments designed to determine if, in skins bathed with $\mathrm{SO}_{4}$-Ringer, net water flow $\left(J_{w}\right)$ could be diminished by agents added to the outer medium. Toad skins were mounted inbetween glass chambers and exposed to a standard osmotic gradient of $200 \mathrm{mOsm}$. $J_{\mathrm{w}}$ was continuously monitored by automatic, volumetric techniques, Exposure to $1 \mathrm{mM} \mathrm{HgCl}$ or $\mathrm{CH}_{3} \mathrm{ClHg}$ (external side) led to the typical anion-induced $J_{\mathrm{w}}$ changes. Re-exposure to $\mathrm{Hg}$ caused a mild $(10 \%)$, although significant $(\mathrm{N}=8, \mathrm{P}<0.001)$, fall in $J_{\mathrm{w}}$. Since $\mathrm{CuSO}_{4}$ is a SH-reagent like $\mathrm{Hg}$, we looked at the effects of $\mathrm{CuSO}_{4}$ $(1 \mathrm{mM})$. There was a $68 \%$ reduction of $J_{\mathrm{w}}(\mathrm{N}=7, \mathrm{P}<0.001)$, an effect totally reversible by simple washing of the skins. Likewise, addition of dithiothreitol (DTT, $5 \mathrm{mM}$ ) in the presence of $\mathrm{Cu}$, caused a $90 \%$ reversal of the Cu effect ; there was no change in $J_{\mathrm{w}}$ if DTT was given before $\mathrm{Cu}$, or if both agents were added simultaneously. Finally, in $\mathrm{Hg}$-treated, glutaraldehyde-fixed skins, $1 \mathrm{mM}$ and $5 \mathrm{mM} \mathrm{CuSO}_{4}$ caused $51 \%$ and $92 \% J_{W}$ decrements, respectively, that were totally reversible. In conclusion, $\mathrm{Cu}$ causes a DTT-sensitive inhibition of $J_{w}$ in $\mathrm{Hg}$ treated skins. Further work is needed to establish if the $\mathrm{Cu}$ effect is exerted on SH-groups of apical aquaporins of toad skin.

\section{$515-43$}

\section{THE KLUYVEROMYCES LACTIS GENE ENCODING THE PHOSPHATIDYLINOSITOL SYNTHASE: PRIMARY STRUCTURE AND EXPRESSION IN SACCHAROMYCES CEREVISIAE}

Dominique Schaller, Monique Belet, Muslema Nazeri and Jacques Deshusses. Département de Biochimie, Université de Genève, $\mathrm{CH}-1211$ Genève 4.

The Kluyveromyces lactis gene encoding the phosphatidylinositol synthase (PIS-KL) was isolated from a genomic library by low stringency hybridization. The Saccharomyces cerevisiae PIS gene was used as a probe. DNA sequence analysis revealed an open reading frame coding for a 225 amino acids protein that is $60 \%$ identical to the $S$. cerevisiae PIS. The most conserved region includes the "signature" of the CDP-Alcohol Phosphatidyltransferases. A yeast replicating plasmid containing the PIS-KL gene was constructed. We used it to transform a $S$. cerevisiae strain, which is mutant for the PIS gene and has an altered $K_{m}$ for inositol. The transformed strain presents a complete restoration of the enzyme activity up to the wild type level.
S15-44

\section{THE PHYLOGENETIC ORIGIN OF PC-645 $\alpha$-SUBUNITS}

Walter A. Sidler, Institut für Molekularbiologie und Biophysik, ETHHönggerberg, $\mathrm{CH}-8093$ Zürich, Schweiz.

The amino acid sequences of the PC-645 $\alpha$-subunits and other cryptomonad biliprotein $\alpha$-subunits pose the most intriguing question about the phylogenetic origin of these unique chromopeptides. Cryptomonad PC-645 $\alpha$-subunits seem not to be closely related to the known phycobiliproteins, linker polypeptides (LPPS), BPE or any other light-harvesting chlpolypeptides from the thylakoid. For most of them the number of identical amino acid residues was $15 \%-20 \%$. Sequence alignment of C-PE associated LPPs with $\gamma$-subunits from cyanobacteria and red algae and the cryptophytan $\alpha$-subunits however show that the identity between $\mathrm{C}$ PE associated LPPs and cyanobacterial $\gamma$-subunits is still significant, whereas it reaches the limit of significance between cyanobacteril and eucaryotic $\gamma$-subunits. Nevertheless it has to be assumed that $\gamma$-subunit derive from cyanobacterial C-PE associated LPPs as well as cryptomonad $\alpha$-subunits may derive from $\gamma$-subunits. Whereas the the phycobiliprotein $\alpha$ - and $\beta$ subunits remained structurally conservative during evolution (e.g. $-70 \%$ identity between cyanobacterial and red algal PE-subunits) the LPPs and $\gamma$-subunits show drastical changes in their chromophore binding domains.

\section{S15-45}

\section{STRUCTURAL MOTIFS IN CHLOROPHYLL PROTEINS} FUNCTIONAL AND EVOLUTIONARY IMPLICATIONS Brunisholz, R.A., Institut für Molekularbiologie und Biophysik, ETH-Zürich, $\mathrm{CH}$-8093 Zürich

Functional and evolutionary relationships of the components of the primary events in photosynthesis have been traced using amino acid sequence comparison. Basically, the question was posed regarding the interrelationship of the apoproteins of different photosynthetic organisms which gather light and/or separate charges across membranes. Do their protein-chemical structure and organisation decipher us certain clues and parts of the path by which they have been selected and derived from a possible primordial reaction centre polypeptide? Within that context the light-harvesting- and energy transfer systems of purple bacteria offered as ideal model components. A data set of more than 70 apoprotein sequences has pointed to some keystructural elements which have been partially verified as such by limited proteolysis and genetic engineering. A careful inspection of the determined bacterial antenna-structures revealed some remarkable similarities to eukaryotic antenna and reaction centre apoproteins indicating possible basic structural motifs for complexing pigment molecules, like chlorophylls or bacteriochlorophylls in very distant representatives of phototrophs.

\section{S15-46}

DISREGULATION OF HUMAN PNS MYELIN PROTEINS EXPRESSION IN NEUROPATHIES ASSOCIATED WITH ANTIMYELIN ANTIBODIES. J-M, Gabriel ${ }^{*}$, B. Erne*, G. C. Miescher*, J. Ulricht, C. Vital and A. J. Steck*. Department of Research and Neurological Clinic*, Institute of Pathology ${ }^{\dagger}$, Basel, Switzerland, Service d'Anatomie Pathologique ${ }^{\circ}$, Hôpital Pellegrin, Bordeaux, France

A number of glycoproteins of the nervous and/or immune system that are involved in cellular recognition and/or adhesion share the common L2/HNK-1 carbohydrate structure. In a large proportion of patients with peripheral demyelinating neuropathy associated with paraproteinemic gammopathy (PPN), monoclonal lgM antibodies (M$\mathrm{IgM}$ ) react with the $L 2 / H N K-1$ determinant of $M A G$ and $P_{0}$. We plan to test the hypothesis that anti-MAG M-lgM autoantibodies may alter the expression of HNK-1 bearing myelin proteins such as MAG, PO, MOG, NCAM or PMP22. By using monoclonal antibodies to myelin proteins on tissue sections, preliminary results indicate that MBP protein content seems to be strongly downregulated, whereas GalC and Si00 protein do not appear to be affected by the loss of myelin in PPN nerves. GFAP protein expression appear to be upregulated in demyelinating biopsy specimens from PPN patients with anti-MAG M-lgM gammopathy. In conclusion, the expression of some L2/HNK-1 negative proteins might be disregulated by the presence of circulating anti-MAG M-IgM in the serum of PPN patients. 


\section{PROTEOLYTIC TREATMENT OF CHLOROSOMES FROM CHLOROELEXUS AURANTIACUS IN 1-HEXANOL GIVES RISE TO GIANT CIRCULAR DICHROISM} Institut für Molekularbiologie und Biophysik, ETH-Hönggerberg CH-8093 Zürich, Switzerland

The green photosynthetic bacterium chloroflexus aurantiacus has a strong near infra-red absorption maximum at 740 nm with a characteristic S-shaped circular dichroism (CD) signal. The absorption band is caused by the antenna system, the chlorosome, which comprises several thousands of bacteriochlorophyll $c$ molecules and three predominant polypeptides with the $\mathrm{M}_{\mathrm{r}} 18,11,5.7 \mathrm{kDa}$. Treatment of isolated chlorosomes with proteinase $K$ in saturating 1 -hexanol concentrations gave rise to a very strong $C D$ signal due to aggregated Bchl C. BY comparison of proteinase $K$ induced effects to trypsin effects we showed that the $5.7 \mathrm{kDa}$ polypeptide has a structural role in the organisation of $\mathrm{Bchl} c$ in the chlorosome.
Lehmann, R.P., Brunisholz,R.A. and Zuber, H.

THE C-DNA OF CH-L1, A NOVEL MEMBER OF THE L1CAM IMMUNOGLOBULIN SUPERFAMILY IN MICE

Holm, J., Hillenbrand, R., Steuber, V., Montag, D., Moos, M. and Schachner, M., De partment of Neurobiology, Swiss Federal Institute of Technology, Hönggerberg, CH-8093 Zürich

Diverse cell surface molecules of the nervous system play an important role in specifying cell interactions during development. With the aid of a polyclonal antibody against $\mathrm{Ll}$, a $1.8 \mathrm{~kb} \mathrm{CDNA}$ clone closely related to $\mathrm{L} 1$ ( $\mathrm{CH}-\mathrm{Ll}$ ) was isolated from a $\lambda$ gt1l library derived from poly $(\mathrm{A})^{+} \mathrm{RNA}$ of day 8 mouse brain. Using this CDNA as probe the remaining CDNA S' sequences were cloned out of a plasmid library constructed from postnatal day 6-14 mouse brain poly (A)+ RNA. Two independent full length clones of 4.2 and $4.4 \mathrm{~kb}$ encompassing the entire coding region of CHL1 were isolated. These represent putative alternatively spliced mRNAs. The deduced primary struc ture of CHL1 reveals that, like the cell adhesion molecules (CAMs) mouse L1, chicken Ng-CAM and Nr-CAM (BRAVO), CHL1 is composed of six Ig-like domains, a transmembrane domain, and a short cytoplasmatic region. In contrast to the other $\mathrm{L} 1$ family members, only four and a half instead of five fibronectin type LI-like repeats are found in CHL1. The fibronectin type III-like repeats were expressed in $E$. coli and the protein fragment was used for immunization. As observed for $\mathrm{L} 1, \mathrm{Ng}-\mathrm{CAM}$, and $\mathrm{Nr}-\mathrm{CAM}, \mathrm{CHL} 1$ is susceptible to proteolytic cleavage. Bands of $185 \mathrm{kD}$, $165 \mathrm{kD}, 125 \mathrm{kD}$, and $50 \mathrm{kD}$ could be detected by Western blot analysis. By in situ hybridization, a predominant expression by neurons was found in the adult mouse brain. Western blot analysis showed expression of CHLl protein in brain and heart, but not lung, kidney and intestine. In liver $250 \mathrm{kD}$ immunoreactive band was found. The relationship of CHL1 to molecules known to be involved in cell adhesion and neurite outgrowth, combined with its pattern of expression, suggests a role for this molecule in cell interactions during neural development.

\section{$515-48$}

MOLECULAR CLONING AND CHARACTERIZATION OF A HUMAN LIVER NA ${ }^{+}$BILE ACID COTRANSPORTER B. Hagenbuch and P.J. Meier. Division of clinical Pharmacology and Toxicology, Dept. of Medicine, University Hospital, 8091 'ürich We have isolated a human liver $\mathrm{Na}^{+}$-taurocholate cotransporting polypeptide (NTCP) by screening a human liver CDNA library with a rat CDNA probe derived from Ntcp (PNAS 88, 10629, 1991). The cDNA codes for a protein of 349 amino acids which shows $77 \%$ amino acid homology with the rat Ntcp. Expression of NTCP in $X$. Iaevis oocytes resulted in $\mathrm{Na}^{+}$-dependent bile acid uptake which exhibited saturability with an apparent $\mathrm{Km}$ for taurocholate of $6 \mu \mathrm{M}$. NTCP mediated taurocholate uptake into oocytes was inhibited by all major bile acid derivatives, bumetanide and bromosulphophthalein. Southern blot analysis of genomic DNA from a panel of human/hamster somatic cell hybrids mapped the human NTCP-gene to chromosome 14. In conclusion, these studies supplement the previously suggested selective mammalian distribution of the Ntcp gene family and provide the possibility for direct molecular characterization of human bile acid transporter genes.

\section{S15-49}

CHANGES IN WATER PERMEABILITY INDUCED BY SEROSAL ANISOSMOTIC MEDIA IN Hg-TREATED TOAD BLADDERS.

Jaquet P. \& de Sousa R.C., Dept of Physiology, C.M.U., Faculty of Medecine, 1211 Geneva 4, Switzerland

Work from this laboratory revealed that in $\mathrm{Hg}$-treated, glutaraldehydefixed toad bladders, the water permeability coefficient $\left(P_{f}\right)$ is high in $\mathrm{SO}_{4}$-Ringer and low in $\mathrm{Cl}$-Ringer ; in all these studies the osmolality of the serosal medium was 220 mosm. As an attempt to determine the mechanism(s) underlying such $P_{f}$ changes, experiments were carried out with bladders whose serosal surface was exposed to iso-, hypo- or hyperosmotic media ; the outer surface was always exposed to a hyposmotic solution ( $22 \mathrm{mOsm})$. Net water flows were measured continuously with automatic, volumetric techniques. After exposure to $1 \mathrm{mM} \mathrm{CH} \mathrm{ClHg}_{3}\left(10^{\circ}\right.$, outer medium) followed by fixation with $0.25 \%$ glutaraldehyde ( 5 , serosal medium), the bladders were thoroughly washed. In $\mathrm{SO}_{4}-\mathrm{Ringer}, \mathrm{P}_{\mathrm{f}}(\mu \mathrm{m} / \mathrm{sec})$ was as follows $(\mathrm{N}=6): 115.3 \pm 13.7$

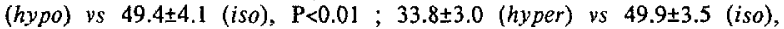
$\mathbf{P}<0.001$. In $\mathrm{Cl}$-Ringer, $\mathrm{P}_{\mathrm{f}}$ was $57.8 \pm 11.4$ (hypo) vs $17.0 \pm 3.0$ (iso), $P<0.01$; and $16.7 \pm 1.9$ (hyper) vs $18.2 \pm 3.5$ (iso), NS. A similar pattern of results was observed in bladders first challenged with vasopressin, fixed with glutaraldehyde and finally exposed to $\mathrm{Hg}$. In conclusion, anisosmotic serosal media caused $P_{f}$ changes that suggest a modulation of the apical water channels permeance by cell volume and/or intracellular osmotic stimuli

\section{S15-51}

GENETIC CHARACTERIZATION OE A CB-TYPE CYTOCHROME OXIDASE IN RHODOBACTER CAPSULATUS

Linda Thöny-Meyer, Christoph Beck and Hauke

Hennecke, Mikrobiologisches Institut ETH,

The genes coding for a new type of haem/copper oxidase, a $c b$ oxidase, were cloned Erom the photosynthetic bacterium Rhodobacter capsulatus and sequenced. Four genes were identified whose products shared homology with the membrane-bound subunits FixN, Fixo, FixQ and Fixp of rhizobial alternative oxidases. FixN is a b-type cytochrome, Fixo is a monohaem- and FixP a dihaem cytochrome $c$. The function of the small Fixg protein is unknown. Structural and Eunctional predictions as weII as mutant and gene expression analyses will be presented.

\section{S15-52}

MOLECULAR PROPERTIES OF THE Na+-DEPENDENT TAUROCHOLATE COTRANSPORTING POLYPEPTIDE (Ntcp) OF RAT IIVER

B.Stieger, B.Hagenbuch, L.Iandmann and P.J.Meier Div.Clin. Pharmacol.Toxicol., University Hospital, zürich/Switzerland, and Dept. of Anatomy, University of Basel, Basel/Switzerland We wanted to determine the molecular weight (MW) of Ntcp and its topology in the plasma membrane (PM) of rat liver. A rabbit antiserum generated against the c-terminus of Ntcp was used to determine its $M W$ on Western blots and its subcellular distribution in liver and in hepatocytes by immunofluorescene (IF). Site directed mutagenesis and deletion experiments were used to determine the $\mathrm{N}$-linked glycosylation sites. Ntcp encodes a $50 \mathrm{kD}$ protein in rat liver PM vesicles. The basolateral localization of Ntcp in liver was confirmed by IF. Ntcp could only be immunostained in permeabilized hepatocytes suggesting an intracellular localization of the cterminus. CNDA constructs showed the Ntcp is glycosylated at positions 5 and 11 . The data show a selective basolateral expression of Ntcp in rat liver and they suggest that the two ends of Ntcp are located on opposite sides of the PM.
Schmelzbergstr. 7, 8092 Zürich 


\section{S15-53}

LOCALIZATION OF NUCLEOSIDE PHOSPHATASE ACTIVITIES ON 2D-PAGE OF CHAFS-SOLUBILIZED PIG ZYMOGEN GRANULE MEMBRANES Soriani, M. and Ereiburghaus, A.U.

Deptment of Internal Medicine, University Hospital,

H LAB 8, CH-8091 Zürich, Switzerland

Zymogen granule membranes (2GM) are known to contain proteins with nucleoside phosphatase activity, but their exact nature and function are unknown. The activities require $\mathrm{Ca}^{++}$and $\mathrm{Mg}^{++}$and are sensitive to ATPase inhibitors but not to inhibitors of Na/K-ATPase activity. The aim of this study was to isolate individual proteins of pig pancreatic ZGM, to attribute enzymatic activity to individual proteins and to characterize them with various substrates (GTP, ATP, AMP etc.), inhibitors (AMP-CP, AMP-CPP, GTPgS etc.) and lectins (MAA, DSA, GNA etc.). We have subjected highly purified ZGM solubilized in CHAPS to IEF on Immobiline Dry strips (Pharmacia) with a pH range $3-10.5$ (1st dim.). These strips were then electrophoresed in a polyacrylamide gradient gel containing $0.2 \%$ CHAPS and Tris/HCL at $\mathrm{pH} 9.5$ (2nd dim.). Nucleoside phosphatase activity was detected histochemically in the $2 \mathrm{D}$ gel by incubation with substrate in the presence of lead nitrate. Focussed strips were also subjected to SDS-PAGE for comparison. Several proteins showed strong activity to GTP, ATP and ITP. The role of these phosphatases are discussed in the context of the role of GTP-binding proteins and the de-/phosphorylating events on the $z G M$ in intracellular targeting and exocytosis.

\section{S15-54}

Cloning and Expression of a Renal Na/Pi Transport SYSTEM FROM FLOUNDER (PSEUDOPLEURONECTES AMERICANUS) Andreas Werner ${ }^{*}$, Jürg Biber ${ }^{\circ}$, Heini Murer ${ }^{\circ}$, and Rolf K.H. Kinne

*MPI for molecular Physiology, Rheinlanddamm 201, D-44139 Dortmund ${ }^{\circ}$ Institute of Physiology, University of Zurich, Winterthurerstr. 190, CH-8057 Zürich In contrast to mammalian kidneys where inorganic phosphate $\left(\mathrm{P}_{\mathrm{i}}\right)$ is reabsorbed unidirectionally, flounder renal epithelial cells both reabsorb and secrete $P_{i}$. In order to investigate on a molecular level the cellular $P_{i}$ handling we have cloned a reabsorptive $\mathrm{Na} / \mathrm{P}_{\mathrm{i}}$ transport system from flounder renal tubules. Based on homology with the cloned $\mathrm{Na} / \mathrm{P}$ cotransporter from rat we have isolated a cDNA clone (flounder NaPiII, $2424 \mathrm{bp}$ ) encoding for a protein of 637 amino acids. In the eight transmembrane spanning domains the protein is $78 \%$ identical with the corresponding system from rat kidney, in the hydrophilic regions only $30 \%$. Expression of in vitro transcribed RNA in Xenopus oocytes specifically stimulated Na-dependent $\mathrm{P}_{i}$ transport. Binding properties of $\mathrm{Na}$ and $\mathrm{P}_{i}$ as well as the $\mathrm{pH}$-dependency were similar to the ones found in mammalian systems (rat, human, OK cells). By Northern analysis three transcripts, $1.9,2.7$, and $4.2 \mathrm{~kb}$ in length, could be detected. RNA from flounder renal tubules contained all of the transcripts, intestinal RNA only the two lower bands, and non-epithelial tissue from kidney expressed only the $1.9 \mathrm{~kb}$ transcript. Mammalian systems share only the $2.7 \mathrm{~kb}$ transcript but not the related species. The close functional relationship of flounder NaPi-II with the known $\mathrm{Na} / \mathrm{P}_{\mathrm{i}}$ transport systems and the pronounced differences in primary structure provide the basis for structure function analysis of $\mathrm{Na} / \mathrm{P}_{\mathrm{i}}$ cotransport.

\section{$\$ 15-55$}

AGONIST-INDUCED PHOSPHORYLATION OF THE $\alpha 1 B$ ADRENERGIC RECEPTOR DOES NOT INVOLVE PKC AND IS NOT REQUIRED FOR RECEPTOR DOES NOT INVOLVE PKC AND

Lattion A.L. and Cotecchia S. Institut de Pharmacologie et Toxicologie, rue du Bugnon 27, $\mathrm{CH}-1005$ Lausanne

Phosphorylation of the adrenergic receptors $(A R)$ is implicated in the regulation of their biochemichal function.

We previously showed that in smooth muscle cells, the $\alpha 1$-AR is rapidly phosphorylated following exposure to agonist. To understand this biochemical phent, we investigated the phosphorylation of the $\alpha 1 \mathrm{~B}$ adrenergic receptor stably event, we investigated the phosphorylation of the $\alpha$ T expressed in Rat1 fibroblasts $(3.3 \mathrm{pmol} / \mathrm{mg}$ prot.). The a $1 \mathrm{~B}-\mathrm{AR}$ was solubilized from 32 -labeled Rat cells and immunoprecipitated with antibodies raised again the $\mathrm{N}$-terminus part of the receptor. Treatment of cells with $100 \mu \mathrm{M}$ epinephrine
showed a rapid and significant increase in receptor phosphorylation above basal. showed a rapid and significant increase in receptor phosphorylation above Treatment of cells with a specific pKC inhibitor (RO-318220) did not affect agonist-promoted phosphorylation while it completely abolished phorbol esterinduced receptor phosphorylation. This strongly suggests that $\mathrm{pKC}$ is not involved in agonist-induced $\alpha 1 \mathrm{~B}-\mathrm{AR}$ phosphorylation.

The a1B-AR contains several putative phosphorylation sites in both its third intracellular loop and C-terminus tail. To investigate the role of these domains, a truncaled receptor (lacking its last 147 amino-acids) was constructed and stably expressed in Rat cells (1.3 pmolimg prot). This receptor mutant displayed similar expressed in Rat celis (1.3 pmol/mg pro.). This receptor mutant displayed similar ligand-binding properties and ability to activate phospholipase $C$ as compared to the wild-type receptor. Phosphorylation experiments revealed that this mutant not at all phosphorylated, neither by agonist nor by phorbol esters. Agonis induced decrease in cell surface receptors, measured by binding of the antagonist ${ }^{3} \mathrm{H}$-prazosin at $4^{\circ} \mathrm{C}$, was however similar for both the wild-type and mutant receptor. This suggests that loss of phosphorylation sites does not affect receptor internalization.

\section{S15-56}

\section{Properties of the scrapie prion protein: quantitative analysis of protease resistance}

Bruno Oesch", Margit Jensen ${ }^{\circ}$, Povl Nilsson ${ }^{\circ}$, Jens Fogh ${ }^{\circ}$

*Institut für Hirnforschung, Universität Zürich, August-Forelstrasse 1, 8029 Zürich; 'Novo Nordisk, Gentofte, Denmark

The disease-specific isoform of the prion protein ( $\mathrm{PrPSc}$ ) is an essential part of the infectious particle causing scrapie or BSE. PrPSC differs from PrP of normal animals ( $\mathrm{PrPC}$ ) by its relative protease resistance. The physical nature of this difference is still unknown. Here we analyzed the protease-resistance of PrPSC quantitatively. PrpSo was rendered completely protease-sensitive at alkaline $\mathrm{pH}$ or in guanidinium thiocyanate $(>1.5 \mathrm{M})$. Denaturation in $2 \mathrm{M}$ guanidinium thiocyanate (GdnSCN) completely abolished protease resistance of $\mathrm{PrPSc}$ within $15 \mathrm{~min}$ while denaturation in $6 \mathrm{M}$ urea showed a much slower time course. Denaturation curves were used to calculate the Gibbs free energy $\left(\Delta G_{D}\right)$ in dependence of GdnSCN or urea at different concentrations. The linear relationship between $\Delta G_{D}$ and the denaturant concentration is suggestive of a two state model involving the conformational change of a single protein domain. This is also reflected in the small number of side chains (11.6) additionally exposed to the solvent upon conversion of PrPSc to its proteasesensitive isoform. Our results suggest that minor rearrangements of the structure of PrPSc are sufficient to abolish its protease resistance.

\section{S15-57}

TROPHIC ACTIVITY OF THE AMYLOID $\beta / A 4$ PROTEIN PRECURSOR. Jean-Marc Roch and Tsunao Saitoh. Dept. of Neurosciences, University of California San Diego, La Jolla, CA 92093, USA

The amyloid $\beta / A 4$ protein precursor (APP) is a transmembrane protein expressed in different isoforms throughout the organism. Our work has consisted in a detailed study of the structure and biological function of APP. Specifically, we have shown that the secreted form (sAPP) of APP-695 is involved in the growth regulation of fibroblasts, and also stimulates neurite extension in $\mathrm{B} 103$ neuroblastoma cell line. Functional mapping studies have shown that the domain responsible for both the growth-regulating and the neurotrophic activities of sAPP- 695 is the RERMS site, Arg-328 to Ser-332. The presence of sAPP binding sites on the surface of B103 cells (through the active RERMS site) indicate that the neurotrophic activity of SAPP is mediated by a receptor, and the subsequent activation of a signal transduction mechanism. In addition, we have shown that the RERMS site is also involved in the neuronal survival properties of SAPP-695. Finally, we have initiated an in vivo study of APP biological function, and shown that a peptide representing the neurotrophic domain of SAPP-695 can strengthen memory retention in rat through stabilization of synaptic structures in the frontal cortex. This work was supported by grants from the Swiss National Science Foundation (823A-028366), and NIH (AG 05131).

\section{$\mathrm{S} 15-58$}

EVIDENCE FOR LYSOSOMAL PROCESSING OF HUMAN LACTASE PHLORIZIN HYDROLASE EXPRESSED IN COS-1 CELLS

Wüthrich, M., and Sterchi, E., Institut für Biochemie und Molekularbiologie, Universität Bern.

Human intestinal lactase-phlorizin hydrolase (LPH) is synthesized as a precursor molecule (pro-LPH), which undergoes proteolytic processing during maturation. LPH expressed in COS-1 cells was enzymatically active. The electrophoretic properties of LPH-species synthesized by transfected COS-1 cells correspond to those in organ cultured human intestinal explants, in Caco-2 celis and in transfected MDCK cells. They included the pro-LPH and a proteolytically processed form, which had similar electrophoretic properties to the mature enzyme. The appearance of the putative mature form was inhibited by Brefeldin $A$, ammonium chloride and chloroquine. In addition, only complex glycosylated pro-LPH (pro$\mathrm{LPHC}$ ) was inserted into the cell surface membrane. These data indicate that in COS-1 cells pro-LPH is inserted into the cell membrane, is internalyzed and enters lysosomes where proteolytic events lead to the appearance of a mature-like enzyme. 
S15-59

ASSEMBLY AND TRANSPORT OF PABA PEPTIDE HYDROLASE ALPHA AND BETA SUBUNITS IN COS-1 CELLS Eldering, J.A., Grünberg, J. and Sterchi, E., Institut für Biochemie und Molekularbiologie, Universität Bern.

Paba peptide hydrolase (PPH) (EC 3.4.24.18), a metalloendopeptidase from epithelial cells of human small intestinal mucosa, consists of two subunits, $\alpha$ and $\beta$, which form homodimers and ofigomers. cDNA cloning has revealed homologies with developmentally important proteins from different species and has led to the definition of a new family of metalloendopeptidases, the astacin family (J. Biol. Chem. 266, 21381, 1991). Here, we report on the expression of the CDNAs of PPH $\alpha$ and PPH $\beta$ in COS- 1 cells. When expressed on its own, PPH is synthesized in a core glycosylated form only, suggesting that it is transport-incompetent and not able to exit the ER. Immuno-fluorescence studies have confirmed that PPH $\alpha$ is not expressed on the surface of COS-1 cells. $\mathrm{PPH} \beta$, on the other hand, matures and is transported to the cell surface. When the two subunits are coexpressed, PPH $\alpha$ can also be detected on the cell surface, suggesting that a heterooligomeric assembly is necessary for the surface expression of PPH $\alpha$. Truncated forms of both PPH $\alpha$ and PPH $\beta$ could be detected in the medium of transfected COS-1 cells.

\section{S15-60}

RADIOAUTOGRAPHIC LOCALIZATION OF NUCLEAR $3,5,3^{\prime}$ TRIIODOTHYRONIN RECEPTORS IN RAT DORSAL ROOT GANGLIA AND SCIATIC NERVE.

Barakat-Walter, I., and Droz, B. Institut d'Histologie et d'Embryologie, Faculté de Médecine, rue du Bugnon 9, CH - 1005 Lausanne.

In our previous work, we demonstrated by immunocytochemical reaction that sensory neurons expressed nuclear triiodothyronin receptors (NT3R) in embryonic as well as adult rats. In contrast, in sciatic nerve, Schwann cells exhibited only transient NT3R immunoreactivity from E17 to postnatal day 10 . In the present work, we attempt to demonstrate by radioautography that the detected NT3R are effectively the binding sites of [1251] labeled triiodothyronin. Cryostat sections of dorsal root ganglia (DRG) or sciatic nerve were incubated with $0.1 \mathrm{nM}$ of $\mathrm{L}, 3,5,3^{\prime}-[125 \mathrm{I}]-$ triodothyronin $2200 \mathrm{Ci} / \mathrm{m}$ mol (NEN), while the controls were incubated with a large excess $(1 \mu \mathrm{M})$ of unlabeled triiodothyronin $\left(\mathrm{T}_{3}\right)$. In adult rat $D R G$, radioautographs revealed that silver grains were exclusively restricted to neuronal cell bodies, while the Schwann cells ensheathing the axons were free of significant label. In newborn rat sciatic nerve, silver grains accumulated over Schwann cells; in contrast in adult rat sciatic nerve, Schwann cells were free of label. In control DRG or sciatic nerve sections incubated in large excess of unlabeled $T_{3}$, all the radioautographs exhibited only scattered silver grains.

In conclusion, our results show that sensory neurons and Schwann cells are able to express functional NT3R which bind thyroid hormones (SNF 31. 33671-92).

\section{S15-61}

NEUROTROPHIN-4/5 PROMOTES DIFFERENTIATION AND SURVIVAL OF STRIATAL GABAERGIC NEURONS IN CULTURE. Hans R. Widmer and Franz F. Hefti; Andrus Gerontology Center, University of Southem California, Los Angeles, CA 90089 and Institute of Anatomy, University of Berne, $\mathrm{CH}-3012$ Beme.

Cultures of dissociated striatal neurons prepared from fetal rats were grown in the presence of neurotrophin-4/5 (NT-4/5) as well as the other known neurotrophins, NGF, BDNF and NT-3. We found that acute administration of NT-4/5 to seven days old cultures stimulates the hydrolysis of phosphatidylinositol, an event involved in neurotrophin signal transduction Growth of striatal cultures in presence of NT- $4 / 5$ resulted in increased cell survival as indicated by elevations in total cell number, protein content and number of viable cells. NT-4/5 exposure increased GABA uptake and staining intensity in GABA immunocytochemistry in these cultures indicating a trophic action on GABAergic neurons. To further identify responsive cell populations we analyzed for calretinin, a calcium binding protein known to colocalize with GABA in a number of neuronal cells. NT-4/5 strongly increased the number of calretinin positive cells in cultures prepared from rats of embryonic day 15 , as well as calretinin levels determined by Westem blot analysis. When cultures were prepared from embryonic day 18 rats, NT-4/5 very strongly increased the morphological differentiation of calretinin positive cells. While all effects produced by NT-4/5 were mimicked by BDNF with similar potency, NT-3 was found to be only marginally effective. Our findings identify NT-4/5 as a potent neurotrophic factor for striatal GABAergic neurons.

\section{S15-62}

Characterization of the matureprecursor glycophospholipid for glycosylphophatidylinositol anchors of Saccharomyces cerevisiae.

György Sipos and A. Conzelmann

From the Institute of Biochemistry, University of Fribourg, Pérolles, $\mathrm{CH}$ 1700 Fribourg, Switzerland

Many attempts to isolate the mature yeast precursor GPI glycophospholipid ready to be attached to newly translated proteins for GPI-anchoring have failed although such precursors were previously identified in mammalian cells and protozoa. Here we show that metabolically labeled glycolipids of the expected structure can be observed if the incorporation, their accumulation and their extraction are optimized. Two very polar, $\left({ }^{3} \mathrm{H}\right)$-mannose-labeled glycolipids named $\mathrm{CP} 1$ and $\mathrm{CP} 2$ were identified and purified. They were structurally characterized using phospholipase treatments, partial deacylation with methanolic ammonia. hydrofluoric acid dephosphorylation, nitrous acid deamination, acetolysis, exoglycosidase treatments and combinations thereof to produce labeled fragments which could be analyzed by paper chromatography or thin layer chromatography. $\mathrm{CP} 1$ and $\mathrm{CP} 2$ both contain the identical core oligosaccharide Man $\alpha 1,2\left(\mathrm{X} \rightarrow \mathrm{PO}_{4}-6\right) \mathrm{Man} \alpha 1,2 \mathrm{Man} \alpha 1,6(\mathrm{Y} \rightarrow) \mathrm{Man} \alpha$-GlcN-inositol, $\mathrm{X}$ and $\mathrm{Y}$ being hydrofluoric acid-sensitive substituents (most likely ethanolamine and phosphoethanolamine, respectively). The inositol is acylated although no acylinosicol is present on protein-bound yeast GPI-anchors. CP1 and CP2 can also be observed after lableling with $\left({ }^{3} \mathrm{H}\right) m y o$-inositol. The lipid moieties of $\mathrm{CP} 1$ and $\mathrm{CP} 2$ can be completely removed by mild alcaline hydrolysis altough the protein-bound GPI-anchors made by the pmi-40 cells under identical labeling conditions are completely mild base resistant. This finding reinforces the notion that the ceramide moiety typically found on the majority of yeast GPI-proteins are added only after addition of the GPI precursor glycolipid to proteins.

\section{$\$ 15-63$}

The distributions of the Mannose-6-Phosphate/IGFII-Receptor and 2,6 Sialyltransferase in HepG2 cells: No evidence for co-localization by confocal laser scanning microscopy.

E.G. Berger; Institute of Physiology, University of Zurich Switzerland; A. Hille, University of Göttingen; T. Bächi, University of Zurich

The organization of the trans Golgi apparatus (GA) with respect to (recycling) man-6P/IGFII receptor (MPR) and (resident) $\alpha 2,6$ sialyltransferase (ST) has been investigated by double immunofluorescence laser scanning confocal microscopy in HepG2 cells. They were chosen because biochemical evidence suggested sialylation of MPR upon recycling (Duncan \& Kornfeld, J. Cell Biol. 1988, 106, 617-28) implicating colocalization of both proteins. In the steady state ST was confined to the GA whereas total (endogenous) MPR was localized to coarse vesicles without evidence for colocalization by confocal microscopy. Endocytosis of surface-labeled MPR was monitored in presence and absence of Brefeldin A (BFA); Without BFA early endosomes ( 2 ' of warming up) showed no, late endosomes (20') little if any colocalization with ST. In presence of BFA, the ST-compartment disappeared whereas late endosomes persisted with some tubular emanations. Under similar conditions, in absence of BFA, endogenous MPR concentrated in the GA region suggesting co. localization with ST. In presence of BFA the MPR compartment formed a reticulum whereas the ST comparment disappeared. In conclusion, within the limits of the techniques used, exogenous MPR was not detectable in the ST compartment whereas endogenous MPR concentrated in the GA region but obvious co-localization was exceptional. Supported by grant 31-30757.91 of the SNSF to EGB.

\section{S15-64}

Molecular analysis of cell/cell fusion by Herpes simplex virus (HSV)

M.Lingen, T.Seck, K.Weise, D.Falke

Div.exp.Virology, J.Gutenberg-University, 55101 Mainz

Fusion of cells and organelles is a general patho-biological phenomenon: Muscle cells, transport vesicles, Langhans cells and virus induced fusion from within (FFWI, during replication) and without (FFWO, by the particies). In vivo and after virus isolation only little fusion can be seen, only after cultivation fusing HSV can be detected; a selective pressure must be released. 6 syn loci are known on the genome; the syn 3 locus is located inside of the cell in the carboxy terminal part of glycoprotein $B$, all other fusion domains are outside the cell. Fusion inducing mutations are in aa $816,853,854,856,857$ and 872 and were correlated to fusion from within and without as well as to selective Cyclosporin A effects. A model is presented for the 3 syn locus. - By recombination the fusion capacity could be transfected to fusion negative viruses. - HSV penetrates the cellmembrane by fusion by 2 ligand-receptor interactions. FFWO ${ }^{+}$-strains penetrate at $0^{\circ} \mathrm{C}$. Cell/cell fusion was analysed and found to need also 2 receptor-ligand interactions if induced by syn 3 mutations. Timing analysis of fusion events and by certain inhibitors allow further conclusions. 
S15-65

REGULATION OF THE LIGHT-DRIVEN EXCITATION QUENCHING IN THE MAJOR LIGHT-HARVESTING COMPLEX OF PHOTOSYSTEM II (LHCII) BY XANTHOPHYLL CYCLE

Gruszecki, W.I. ${ }^{1}$, Kernen, P. ${ }^{2}$, Krupa, Z. ${ }^{1}$, and

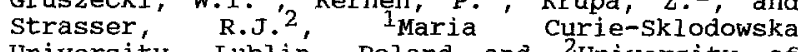
University, Lublin, Poland and ${ }^{2}$ University of Geneva, Bioenergetics Laboratory, CH-1254 JUSSY

In buffer light-driven chlorophyll a (Chla) fluorescence quenching of LHCII isolated from dark-adapted (LHCII-D) or preilluminated (LHCIIL) rye leaves was similar in both preparations, but reversibility of this process within two hours of darkness was slower in LHCII-D. No differences in fluorescence quenching and full reversibility was observed for both LHCII preparations in reverse micellar solution. Xanthophyll/Chl concentrations in LHCII were consi-derably decreased under this conditions. Excitation difference spectra (before and after illumination) of LHCII in asolectin liposomes showed typical changes of energy transfer between carotenoids and Chl. A model is proposed according the xanthophyll cycle controls reversibility of light-driven excitation quenching as well as the rate and extent of quenching in LHCIL.

\section{S15-66}

CD44 EXPAESSION BY HUMAN NEUROBLASTOMA TUMORS AND CEL UNES: RELATIONSHIP TO MYCN AMPUFICATION AND DIFFERENTIATION N. Gross', D. Beck', D. Jackson', C. Beretta' and G. Peruisseau', 'Oncohematology Unit, Pediatrics, University hospital, 1011 Lausanne, Switzerland and 2John Radcliffe Hospital, Oxford, GB

Neuroblastoma (NB) are sympathetic tumors of childhood characterized by MYCN amplification in advanced stages. CD44 standard (CD44s) and splice variants (CD44v) represent a group of surface glycoproteins whose expression has been recently linked to metastasis. We analysed the expression of CD44s and CD44v on NB tumors and cell lines by immunohistology and Northern blot. Results showed high levels of CD44s on $33 / 44$ NB, $6 / 10 \mathrm{NB}$ cell lines and $4 / 4$ ganglioneuromas (mature, benign sympathetic tumors). Lack of CD44s staining was observed on stage IV, MYCN amplified tumors only. In cell lines, expression was not detected on cells with a neuronal phenotype while high levels of CD44s were expressed by cells with a glial differentiation, independently of MYCN amplification. Up-regulation of CD44s was obtained with agents that induce a glial differentiation, while neuronal differentiation by retinoic acid was not accompanied by a change in CD44s expression. No CD44v were detected on tumors or cell lines. We conclude that CD44s expression is down-regulated in a subset of NB cells and tumors and that MYCN product and additional mechanisms responsible for control of differentiation are involved in this regulation. No evidence of a role for CD44v expression in NB metastasis could be demonstrated. (Supported by the Fond National Sulsse de la Recherche Scientifique $\mathrm{nr}$ 32-31005.91)

\section{S15-67}

QUINONE CYTOTORICITY IS MEDIATED THROUGH SELECTIUE INHIBITION OF RDENYLATE CYCLASE $O$. MOULLET and J.-L. DREYER, Institut de Biochimie, Université de Fribourg, CH-1700 Fribourg

cAMP has been recently shown to promote a cell survival response by retarding apoptosis (Berridge, M.V\& al. (1993) Exp. Hematol. 21, 269. 276 ). On the other hand quinones are widely distributed substances of often potential toxicological significance. We have tested the effects of quinones on adenylate cyclase. The enzyme is rapidly inhibited by pbenzoquinone $(I C 50=40-45 \mu \mathrm{M})$ or dichiorophenol-indophenol $(I C 50=200$ $\mu \mathrm{M})$, but 2-substituted quinones are inactive. The $I_{5} C_{50}$ 's are decreased 310 times after membrane solubilization but are not affected by GTP, GDP or analogues nor by cholera and pertussis toxins. The inhibition is not mediated by a G-protein or by the activation of a defined receptor. Quinone inhibition stoichiometrically competes with forskolin activation of adenylate cyclase, equimolar concentrations of both quinone and forskolin restoring the enzyme activity to its basal value. The cytotoxicity of benzoquinone, tested in vivo on Hep-G2, a human hepatocellular carcinoma cell line, could be prevented with forskolin. In plasma membranes quinones tightly bind to only one major and two minor proteins that were purified by PAGE electrophoresis under native conditions. Together these observations indicate that quinone action can be attributed to targetting to a limited number of proteins at the plasma membrane in a highly selective way. By affecting adenylate cyclase, a modulation of the cAMP pool induces apoptosis as a result of the cytotoxicity via.
S15-68

\section{CHARACTERIZATION OF A NEW MYELIN-SPECIFIC GENE}

N.Schaeren-Wiemers and M.E. Schwab

Institut für Hirnforschung der Universität Zürich, August-Forel-Str.1, CH-8029 Zürich

Myelinogenesis is a complex, developmentally regulated process involving coordinate expression of myelination-related proteins. The myelin forming cells are the oligodendrocytes in the central nervous system (CNS) and the Schwann cells in the peripheral nervous system (PNS). We differentially screened a rat postnatal day 16 (P16) spinal cord cDNA library with probes derived from normal (plus probe) and from myelin-free (minus probe) P16 spinal cord mRNAs. We succeded in the isolation of several novel cDNA clones whose cortesponding mRNAs were expressed either selectively by oligodendrocytes or by oligodendrocytes and Schwann cells.

Here we describe one of the cDNA clones (tentatively denominated NS 2) whose mRNA is specifically expressed by oligodendrocytes and Schwann cells. The $3.5 \mathrm{kD}$ transcript is expressed at highest level during myelination and at much lower levels in the adult as it is known for other myelin-specific mRNAs. Sequence analysis of the full length cDNA sequence showed a $50 \%$ identity to the rat liver UDPglucuronosyltransferase family, involved in the detoxification system. The 541 amino acid open reading frame encodes a $62 \mathrm{kD}$ protein with a putative signal peptide and two transmembrane domains, Whether this NS 2 protein is involved in a detoxification reaction or in glycosilation of proteins and lipids with glucuronic acid is under investigation.

\section{S15-69}

COMPARATIVE ANALYSES OF THE GLYKOPROTEIN gC GENE OF BOVINE AND CAPRINE HERPESVIRUSES Monika Engels, Paul Hecht, Eva Loepfe and Mathias Ackermann Institute of Virology, Vet.Med. Faculty, Winterthurerstr. 266a, $\mathrm{CH}$ - 8057 Zürich

The glycoprotein $\mathrm{gC}$ of herpesviruses is known to be non-essential for virus replication. Non-essential herpesvirus proteins are suggested to perform "luxury functions" which may influence the outcome of an infection. We are aiming to analyse the function(s) of gC specified by bovine herpesvirus 1 (BHV1), bovine herpesvirus 5 (BHV5) and caprine herpesvirus 1 (CapHV1). These viruses are closely related but differ in their pathogenic potential. In a first step we have cloned and sequenced the individual genes. The nucleotide and the deduced amino acid sequence homologies of two BHV1 reference strains was found to be $>98 \%$. The sequence homologies between $\mathrm{BHV} 1$ and $\mathrm{BHV} 5$ were $>75 \%$ and those between BHV1 and CapHV1 were $>65 \%$. Comparisons on the amino acid sequence level revealed that the differences were most prominent in the N-terminal parts, whereby the potential signal sequence region might be concerned. The putative glycosylation sites, however, were not affected, and the transmembrane sequences were similar in size and location. In order to characterize the significance of these differences, we are constructing $\mathrm{gC}$-deletion mutants and recombinant viruses carrying a foreign gC gene. The in vitro and in vivo behaviour of these viruses will be compared to the wildtype virus. 


\section{Molecular Biology of Parasites}

S16-01

THE INTERACTION OF TRYPANOSOMA CONGOLENSE WITH

ENDOTHELIAY, CELLS.

Andrew Hemphill, Simon L. Croft, and Carole A. Ross* London School of Hygiene and Tropical Medicine, and *Centre for Tropical Veterinary Medicine, Edinburgh

T.congolense is an African, unicellular hemoflagellate, pathogenic for domestic animals such as cattle. Within the host bloodstream, parasites adhere to the wall of the microvasculature, causing severe organ damage. We have set up and characterized an in vitro assay in order to study the metabolic requirements, receptor-ligand interactions and the ultrastructure of this adhesion phenomenon.our findings suggest that adhesion is an active process requiring metabolic energy on part of the parasite, but is independent of the target cells.We also show that $T$.congolense possess a lectin-like domain localized at specific sites along the surface of the anterior end of the flagellum, which interacts with specific carbohydrate receptors, probably sialic acid and/or $\mathrm{N}$-acetylglucosamine residues, on the endothelial cell surface. This suggests that adhesion is likely to be mediated by a trypanosomal surface component distinct from the variable surface glycoproteins(VSGs). We also suggest that the cytoskeletal protein actin is involved in this process.

\section{S16-02}

ANIIGENIC VARIATION OF GIARDIA LAMBLIA AND INTESTINAL IgA $B$. Gottstein \& Norbert Muller, Institute of Parasitology, University of Berne, Berne, switzerland. Local immune response based upon intestinal parasitespecific $T$ and $B$ cells to Giardia lamblia may be impor tant in parasite clearance. Experimental infections of mice (CR:NIH:S) with G. lamblia (Clone GSM-H7 which exipresses a major $72 \mathrm{kD}$ antigen on its surface recognized by $\mathrm{MAb} G 10 / 4)$ have demonstrated that the parasite undergoe: surface antigenic variation in vivo. Experimental infection of immunodeficient mice (nu/nu mice and scíd mice) and control nu/ + littermates provided insights intio associated immunological mechanisms: dominant surfad epitope-specific sIgA plays a major role in modulating surface antigenic variation, and thymus-dependent $T-1 Y n_{i}$ phocytes in the induction of selfcure. Athymic nu/nu mice (ZU.ICR-strain) were reconstituted with immune Peyer is patch lymphocytes obtained from self-healed littermatle $\mathrm{nu} /+$ mice. Intestinal B-cells from these nu/ + mice as well as from reconstituted athymic nude mice synthesized in vitro parasite-specific IgA. This IgA showed a predominant immunoblot reaction with the major surface antigen ( $\mathrm{a} \mathrm{M}_{\mathrm{r}}$ 72,000 polypep-tide) characterizing the Giardia clone in question. Nu/nu mice reconstituted with immune cells accuired the potential to decrease significantly their intestinal parasite mass. Thus the hypothesis on the causative role of intestinal IgA in the control of intestinal $G$. lamblia infection has found further experimental support.

\section{S16-03}

\section{CHROMATIN DIMINUTION IN ASCARIS LUMBRICOIDES: SEARCHING FOR CONTROL ELEMENTS \\ Stephan Jentsch, Heinz Tobler and Fritz Müller,}

Institute of Zoology, University of Fribourg, Pérolies, 1700 Fribourg

The process of chromatin diminution in the parasitic nematode Ascaris lumbricoides leads to the formation of somatic cells that contain less DNA than the germ-line cells. During this process, which occurs between the third and the fifth embryonic cleavage divisions in five different blastomeres, the termini of the chromosomes are cut off and eventually degrade in the cytoplasm. To analyze this process at the molecular level, we constructed a $\lambda$ Zap somatic telomere library. The analysis of three cloned telomeres and their corresponding germ-line genomic regions revealed that chromosomal breakage takes always place within short specific chromosomal regions (CBRs) and is followed by the addition of the telomeric sequences (TTAGGC)n to the breakage sites. The different $C B R s$ do not crosshybridize to each other. A comparative nucleotide sequence analysis is now being performed to screen for the existence of conserved sequence motifs.

In a parallel approach we analyze the chromatin structure of the CBRs and their flanking regions in developmental stages before and during the elimination process. Putative recognition or regulation sequences important for the elimination process might be recognized as DNasel hypersensitive sites. Once such sequences will be identified, it should be interesting to establish their role in the elimination process and to look for specific binding factors.

\section{S16.04} FROM ASCARIS TO C. ELEGANS: A WAY TO STUDY GENE
STRUCTURE AND FUNCTION

Huang Y-J., Tobler $\mathrm{H}$. and Müller F., Institute of Zoology, University of Fribourg, Pérolles, $\mathrm{CH}-1700$ Fribourg

Chromatin diminution takes place in all presomatic cells of the early embryo of Ascaris lumbricoides and is followed by the addition of many repeats of the telomeric sequence TTAGGC. Chromosomal fragmentation is developmentally regulated and occurs within specific chromosomal regions (CBRs). One of these CBRs (CBR1) was analyzed in detail. A gene located close to the CBR1 encodes a putative GTP-binding protein, whose promoter region is located within a distance of only $2 \mathrm{~kb}$ from the telomeric TTAGGC repeats of the corresponding somatic chromosome. A homologous gene was isolated from C. elegans. Most interestingly, the $C$. elegans GTP-binding protein gene is also located at a telomeric position, namely at the end of chromosome V. In Ascaris, transcripts of this gene are present in all developmental stages, though they seem to be stronger expressed in oocytes and early embryos than in later developmental stages and somatic tissues. A high degree of sequence conservation of these genes between the two nematode species and man (a corresponding partial cDNA clone was identified in a human heart cDNA library) suggests an important biological function. Currently, we are using genetic methods to determine the possible function of this gene in $C$. elegans and to test whether its telomeric localization in both nematode species has any functional importance.

\section{S16-05}

\section{CHROMOBOX (-ES) IN C. ELEGANS}

Agostoni, E., Hill, F.* Wittmann, C. Tobler, H. and Wüller, $F$

Institute of Zoology, University of Eribourg, $\mathrm{CH}$,

*Dept. Of Haematology, University of Cambridge, UK.

The gene polycomo is responsible for the elaboration and maintenance of the expression patters of the homeotic genes during development of Dresophila. It encodes a nuclear protein which is associated with the chromatin together with other proteins of the poiycomb group. The mechanism of gene regulation caused by polycomb seems to be similar to that of the modifier genes of the position variegation effect which also encode structural proteins of the heterochromatin. One of these protetins is HPI which shares with Polycomb a region of similarity at the amino terminus called the chromatin organisation Modifiex domain chromo domain). The particulas Eunctson of the chromo domain is not yet understood, but it seems to be important for the protein-protein interactions in chromatin associates complexes. Furthermore, chromobox containing genes were found in several species, suggesting that they are widespread in the animal and plant kingdoms. Here we show that $\mathcal{C}$. elegans contains at ieast ore chromobox containing gene (cDNA: cm08h7). Length and structure of the putative c. elegans chromo domain proteir aze similar to those of the chromo domain containing proceins of other species. The expression pattern of cmo8h7 is szudied with antibodies raised against the plitative chromo domain protein and by injecting a $\mathbf{s}$-gal fusion construct inco the germ line of $\mathrm{C}$. elegans.

\section{S16-06}

\section{IN VITRO CHROMATIN DIMINUTION SYSTEM FROM ASCARIS LUMBRICOIDES}

L. Magnenat, T. Nilsen*, H. Tobler and F. Müller. Institute of Zoology, University of Fribourg, CH-1700 Fribourg. *School of Medicine, Case Western Reserve University, Cleveland, Ohio 44106, USA.

Chromatin diminution in the parasitic nematode Ascaris lumbricoides is a complex molecular mechanism, involving cleavage of the chromosomes at specific breakage regions, degradation of the eliminated chromatin and new telomere formation in all presomatic cells during the early embryonic development. The aim of the present project is to reproduce this DNA elimination process in vitro, step by step or all in one, by using cell-free extracts of $A$. lumbricoides eggs. Therefore, $4-8$ cell extracts were established and their quality was assessed by the ability to transcribe $5 S$ rRNA genes (pol III) and SL RNA genes (pol II). Faithful extracts were then tested for cleavage activity on DNA substrates derived from different chromosomal breakage regions. Finally, preliminary results revealed a possible non processiv RNAse sensitive telomerase activity in the in vitro extracts, capable of adding specific nucleotide sequences to an oligonucleotide primer containing four repeats of the telomeric sequence TTAGGC. As de novo synthesis of several kilobases of somatic telomere is likely to require a strong telomerase activity, we assume that this $A$ lumbricoides in vitro system is a good tool to isolate the telomerase protein and its corresponding gene or other implicated factors. 
S16-07

SULFOLIPID-BIOSYNTHESIS IN PATHOGENIC AND APATHOGENIC MYCOBACTERIA

A. Kammer, K. Schopfer and T. Burkart

Institute of Medical Microbiology, University of Berne, $\mathrm{CH} 3010$ Berne

A common feature of all mycobacteria - whether pathogenic or not - is their richness and variety of lipids. Among numerous lipids widely distributed, pathogenic mycobacteria exhibit a group of sulfated surface lipids thought to be determinants of virulence. Therefore, we studied sulfolipid-biosynthesis in pathogenic and apathogenic (opportunistic) mycobacteria species by monitoring the kinetics of [ [35 $\mathrm{S}$ ]sulfate incorporation into cellular lipids. Pathogenic mycobacteria (two virulent $M$. tuberculosis strains) showed a marked sulfolipid-synthesis with highest rates during exponential growth, while apathogenic ones (an avirulent $M$. tuberculosis strain and an opportunistic species) manifested negligible incorporation of the label. The partial characterization of the $\left[{ }^{35} \mathrm{~S}\right]-$ sulfated lipids of pathogenic mycobacteria by thin layer chromatographic separation, combined with autoradiographic detection, revealed the presence of several radiolabeled lipid components of different polarities and with altering expressionpatterns during growth. These results strengthen the hypothesis, that sulfolipids are involved in the pathogenesis of mycobacterial disease.

\section{S16-08}

\section{ANALYSIS OF ANTIBODYBINDING LIPIDS} A NOVEL LIPID ANTIGEN IMMUNOASSAY (LAIA)

F. Roth, F. Riniker, K. Schopfer and T. Burkart Inst. Med. Microbiology, Univ. of Berne, CH 3010 Berne It has recently been shown that bacterial lipids cancarry antigenic determinants. The study of antibody specificity to such lipid epitopes in vitro turns out to be difficult since hydrophobic antigens have to react with watersoluble molecules. We have developed a solide phase lipid antigen immunoassay (LAIA) that allows to quantify the antibody reactivities with an array of Iipid antigens derived from nycobacterial cells. Defined amounts of extracted lipids were immobilized as equidistant lines on a nitrocellulose sheet. Small strips of nitrocellulose, each carrying the same sets of lipids were incubated with different human sera. Bound antibodies were then reacted with [125-I]-Iodine labelled antihuman antibodies and visualized by autoradiography. The reaction was quantified by densitometry. Assay conditions were optimized for antigen and antibody concentrations and appropriate incubation times. In addition, the effects of different ingredients (salts, proteins, detergent) on antigen immobilization and epitope accessability were studied. The presented LAIA-system is rapid, sensitive and reproducible. It allows to compare the specificities of different bacterial lipid antigens and to quantify their reactivity with serumantibodies.

\section{S16-09}

\section{STAGE SPECIFIC TRANSCRIPTION AND TRANSLATION IN LEISHMANIA MAJOR}

Tanja Noll, Chantal Desponts, Michel Bernard, Nicole Déglon, Jacques Mauël, Theresa A. Glaser and N. Fasel, Institut de Biochimie, Université de Lausanne.

Within the family Trypanosomatidae, Leishmania is a protozoan pathogen producing a broad spectrum of human disease. Its life cycle is divided in two stages. Promastigote is the flagellated form which colonizes the gut of the sandfly vector, and amastigote is the non-flagellated form that is specialized for growth and survival in the vertebrate host macrophage. To understand gene regulation during the transition between the promastigote and the amastigote stage, we were interested in the characterization of stageregulated genes. We isolated a gene, $\underline{\mathrm{sw} 3}$, from Leishmania major whose mRNA is more abundant in amastigote compared to promastigote. Structural analysis of this gene showed that an additional polypeptide could be encoded by the opposite strand of sw3. We confirmed the presence of an open reading frame on the opposite strand by in vitro translation of the sw3 antisense RNA. In vivo, an amastigote sw 3 antisense transcript and the corresponding polypeptide were also detected suggesting that the $\mathrm{sw} 3$ is transcribed in both directions and that stage specific transcripts and polypeptides could be produced. Analysis of other gene segments tend to indicate that an intensive usage of the Leishmania genome to produce various polypeptides could be a general phenomenon in trypanosomatidae.
S16-10

SEQUESTRATION PLASMODIUM FALCIPARUMHNFECTED ERYTHROCYTES IN SCID MICE

K. Willimann, H. Matile*, N. Weiss and B. A. Imhof**

Schweizer Tropeninstitut, Socinstr.57, 4002 Basel

"PRPI, F.Hoffmann-La Roche, Basel, "*Basel Institute for Immunology, Basel

Cerebral malaria (CM) is a fatal complication of infection by Plasmodium falciparum (Pf). The neurological symptoms which characterize this form of malarial disease are accompanied by the sequestration of Pf-infected erythrocytes (PfE) to specific organs. Adhesion receptors for PfE (CD36, ICAM-1, VCAM-1, E-Selectin and TSP) on the endothelia are inducible by cytokines which are present in severe malaria $(1,2)$. Some features of human Pf malaria are observed in murine malaria, although the pattern of sequestration changes due to the different plasmodial species involved.

In mice, we showed by immunohistochemistry that the expression of VCAM-1 and ICAM-1 is upregulated in brain, lung and heart of Plasmodium bergheiinfected Balb/c and also in LPS-primed SCID mice. In SCID mice injected with labeled human erythrocytes $(E)$, a higher rate of sequestration to brain was observed with PfE than with uninfected E. LPS-priming increased the retention of PfE in the brain and lungs significantly ( $t$ test), but decreased it in the spleen. We conclude that PfE express surface structures which allow them to adhere to the vasculature of the brain more efficiently than uninfected E. LPS increases the number of adhesive molecules on the host endothelium. Interestingly, sequestration in the SCID mouse resembles that of PfE in man.

1 Ch.Ockenhouse et al. 1992.J.Exp.Med.176,pp1183;

2 D. Kwiatkawski et al. 1990,Lancet,336,pp121

\section{S16-11}

\author{
Imaging Bacillus subtilis treated by penicillin using the \\ atomic force microscope \\ B. Fellay, S. Kasas and M.R. Celio
}

Institut d'Histologie, Pérolles, CH-1700 Fribourg, Switzerland

The atomic force microscope is a very convenient tool for studying hard and frat samples with atomic resolution. Biological objects, usually soft and rough, are sometimes difficult to image using this technique. In contrast bacteria, which are too smail to be observed with high resolution using the optical microscope, are hard enough to be observed with an AFM at a relative good level of magnification. This kind of microorganisms can be prepared for AFM imaging using very rapid and simple techniques. This method could be a convenient tool to observe the effect of bioactive substances such antibiotics. We show in this poster an exemple with the action of penicillin on $B$. subtilis.

S16-12

\section{Histone $\mathrm{H} 1$ in procyclic Trypanosoma brucei brucei}

Markus Burria, Wolfram Schlimmea, Bruno Betscharta, Johann Schaller $b$, Herbert Lindner ${ }^{\mathrm{C}}$ and Hermann Heckera a Schweiz. Tropeninstitut, Socinstrasse 57, $\mathrm{CH}-4002$ Basel; b Institut für Biochemie, Freiestrasse 3, CH-3000 Bern; $c$ Institut für Medizinische Chemie und Biochemie, F. Preglstrasse 3, A-6020 innsbruck

The four variants and / or posttranslational modifications of histone $\mathrm{H} 1$ of procyclic Trypanosoma brucei brucei (Protozoa, Kinetoplastida) were purified by HPLC reversed phase chromatograpy and characterized by their amino acid composition and partial amino acid sequence. Differences in sequence of up to $45 \%$ as compared to histone $\mathrm{H} 1$ of higher eukaryotes exist. Alkaline phospatase digestion and analysis of $\mathrm{H} 1$ by means of HPCE was used to demonstrate the presence of phosphorylated modifications. The unique biochemical properties of $\mathrm{H} 1$ of $T$. b. brucei contribute to the structural differences seen in the chromatin of the parasite as compared to that of the higher eukaryote host. 
S16-13

POLYDNAVIRUS (PDV) GENE EXPRESSION IN LEPIDOPTEROUS HOST I.ARVAE

P. Stettler, D.Schümperli and B. Lanzrein Abt. Entwicklungsbiologie, Baltzerstr. 4 , Universität Bern, $\mathrm{Ch}-3012$ Bern

Larvae of the parasitic wasp chelonus inanitus (Braconidae, Hymenoptera) develop inside embryonic and larval stages of the host spodoptera littoralis (Noctuidae, Lepidoptera). Parasitism induces in the host a precocious onset of metamorphosis and developmental arrest in the precocious prepupa. The wasp injects, along with the egg, PDV and venom into the host egg. Injection experiments with PDV and venom revealed that PDV play a crucial role in altering host development and in suppression of the host's immune system. The genome of the PDV of $\mathrm{c}$. inanitus consists of at least 10 segments of double-stranded circular DNA ranging in size from $7-30 \mathrm{~kb}$. We analyzed the expression of viral genes in the course of parasitization with CDNA made from mRNA at various developmental stages of S.littoralis. We also investigated the localization of the expressed viral genes on the various segments.

\section{S16-14}

Alzheimer disease, a neurospirochetosis? Imaging of the suspected bacteria using the atomic force and the scanning electron microscopes.

J. Miklossy and S. Kasas*

University Institute of Pathology, Division of Neuropathology, CH-1005 Lausanne.

*Institute of Histology and General Embryology, University of Fribourg, Faculty of Sciences, $\mathrm{CH}-1700$ Fribourg

Recently bacteria were found in blood and cerebrospinal fluid of neuropathologically confirmed cases of Alzheimer disease.

In addition, these bacteria were isolated and cultured from brain tissue. To identify the infectious agent we used, among other techniques, an atomic force microscope (AFM)

When the isolation fluids derived from the cortex of three Alzheimer's cases were examined with AFM and scanning electron microscopes, we observed bacteria whose size and morphology fit to the order of Spirochaetales. These images are presented and discussed on our poster.

\section{S16-15}

RESISTANCE MECHANISMS IN MURINE ALVEOLAR ECHINOCOCCOSIS fottstein Bruno, Institute of Parasitology, CH-Berne. Alveolar echinococcosis $\mathrm{AE}$ is due to infection with the metacestode (larval stage) of Echinococcus multilocula$r i s$. An immunodominant antigen Em2 of $E$. multilocularis was characterized by MAb-G11. Respective studies reveafed, that (a) antigen expression metacestode stagespeci-fic and (b) that expression was restricted to the laminated layer. The Em2-antigen is a lectin-binding carbohydrate linked to a lipid residue. The "Em2positive" laminated layer proved to be a crucial factor in protecting the parasite from host immune-effector mechanisms: only E. multilocularis larval structures expressing Em2 were able to induce secondary alveolar echinococcosis in rodents. Subsequent experimental stuqies in resistant (C57BL/10) versus susceptible (C57BL/ 6.J and $A K R$ ) mouse strains showed that resistance was as sociated with synthesis of anti-Em2 IgG 3 and $\operatorname{IgG}_{1}$. Susceptibility of C57BL/6J-mice was associated anti-Em2 ${ }^{\prime} g_{2} \mathrm{a}^{-}$and no IgG3. The parasite-specific in vitro lymWhoproliferative immune response remained stationary with time after infection in $\mathrm{C} 57 \mathrm{BL} / 10$ and decreased in Q57BL/6J and AKR mice. Day $30 \mathrm{p} \cdot \mathrm{i} \cdot \mathrm{CD} 4^{+/ C D 8^{-}}$lymphoblast cells dominated in all mouse strains; day $90 \mathrm{p.i}$. an increased number of $\mathrm{CD} 4 / \mathrm{CD} 8^{+}$and $\mathrm{CD} 4^{+} / \mathrm{CD} 8^{+}$cells were obgerved. Only susceptible mice exhibited a significantly decreased response of lymph node cells to conA-stimulation with time p.i. In conclusion, subtypes of parasitegpecific cellular and humoral host immune responses seem to moutuate the elinfcal courises of mürine $\mathrm{AE}$.
S16-16

\section{LEISHMANIA MAJOR TRANSCRIPTS EXPRESSED DURING EARLY INFECTION}

Theresa A. Glaser, Daniel C. Robyr, N. Fasel and Jacques Mauel, Institut de Biochimie, Université de Lausanne

Leishmania species are protozoan parasites causing a spectrum of clinical manifestations in man ranging from cutaneous lesions to morbidity and death. The insect stage (promastigote) and intracellular stage (amastigote) differ in terms of morphology, physiology, antigenicity and gene expression. It is clear that the amastigote stage is uniquely adapted for survival within the inimical environment of the macrophage phagolysosome and that mechanisms regulating these adaptations must be called into play during the early stages of infection when the parasite undergoes transformation. In an effort to elucidate these regulatory mechanisms, we have constructed a "subtracted" cDNA library which is specifically enriched for parasite transcripts expressed during the first 7 hours of infection ("switch" genes). Several clones have been characterized by sequence, Northern, and Southern analysis. Three clones, Sw3, Sw44, and Sw 20 are expressed at a several-fold greater level in the amastigote stage and potentially encode proteins which interact with nucleic acids. Sw3 predicted protein is homologous with histone H1 proteins and Sw44 and Sw20 potentially encode ribosomal proteins. Analyses of transcripts from this library are yielding clues not only to mechanisms underlying the regulation of parasite transformation, but also insights into post-transcriptional and translational control of gene expression in Leishmania.

\section{S16-17}

Replication of Sendai virus RNAs from plasmid DNAs, an open way to negative stranded RNA virus replication and transcription to experimentation by directed mutagenesis.

Calain Philippe and Roux Laurent, Department of Genetics and Microbiology, University of Geneva, Medical School, C.M.U., 9 avenue de Champel, 1211 Geneva 4.

A viral RNA representing the sequence of a defective interfering RNA, naturally selected for efficient replication, i.e. only containing the replication promoter, was cloned under the control of the T7 RNA polymerase promoter. The expressed $T 7$ transcript was then replicated in vivo by supplying in trans the replication functions, equally expressed from plasmids. This system, completely accessible to genetic manipulations, allowed us to define a basic rule directing sendai virus replication, the "rule of six" (Calain and Roux, J.Virol. $67: 4822-4830$, 1993). A second defective RNA, representing a shorter version of the viral RNA, in that it contains not only the replication but also the transcription promoters, was then cloned and replicated in the same system, although with lower efficiency. The replication of this "transcribing" viral RNA was found to obey the "rule of six" as well. Swapping experiments exchnaging regions between replication and replication/transcription promoters were then achieved to define the cissequence requirements needed for efficient replication or for replication itranscription. Results obtained with these chimerical viral RNAs will be presented and their contribution to the comprehension of the paramyxovirus replication and transcription processes

\section{S16-18}

\section{FYN AND ICK ACTIVATION IN TEEILERIA PARVA-} INTECIED T-CELIS

D. Dobbelaere and M. Eichhorn*, Institute of Parasitology, University of Berne, Switzerland and *Kernforschunszentrum, IGEN, Karlsruhe, FRG.

Bovine T-cells that become infected with the intracellular parasite theileria parva undergo lymphoblastoid transformation and acquire the ability to proliferate continuously. They cease to require specific antigenic stimulation, become independent of exogenous growth factors and cause tumors in nude mice. The IL2 and IL2R genes are constitutively expressed and the transcriptional activator NFKB which regulates these two genes is continuously activated. These processes are all strictly dependent on the presence of the parasite in the host cell cytoplasm and cease upon the specific killing of the parasite by the theilericidal drug BW720c. We have shown that the presence of the parasite results in the activation of the protein tyrosine kinases fyn and $l c k$, which can particlpate in early $T$ cell receptor signalling, suggesting that the parasite may use this signal transduction pathway to induce continuous proliferation. 
S16-19

\section{Cysteine proteinases of Leishmania as targets for chemotherapy.}

Jacques Bouvier* ${ }^{\dagger}$ and Judy Sakanari* *Department of Pathology, UCSF School of Medicine, San Francisco, $\mathrm{CA}$, and ${ }^{\dagger}$ Animal Health, Ciba-Geigy, CH1566 St. Aubin, Switzerland.

The overall goal of the study is to apply the structure-based approach to develop lead compounds and design novel enzyme inhibitors as potential therapeutic agents against leishmaniases. In this regard, we have identified and characterized the cysteine proteinases of leishmania major, and showed that they consist of excellent targets for drug design.

We have purified a membrane-bound cysteine proteinase not previously described in other trypanosomatids or protozoans and obtained amino acid sequence information from the purified enzyme. In addition, we have also characterized and purified the soluble cysteine proteinases of the parasite. Consequently, we have isolated two genes encoding the soluble and membrane proteinases. The soluble cysteine proteinase gene occurs in multiple copies of $2.9 \mathrm{~kb}$ with flanking regions of 1.2 and $2.2 \mathrm{~kb}$ and is localized on one chromosome of approximately $440 \mathrm{~kb}$. The membranebound enzyme gene is $3.1 \mathrm{~kb}$ and occurs as a single copy on a chromosome of approximately $1100 \mathrm{~kb}$.

A three dimensional computer model of both genes will be generated and enable us to scan the vast chemical database for new lead componds. We already have identified several cysteine proteinase inhibitors that are effective compounds in killing both promastigote and amstigote stages of the parasite in the micromolar range in vitro without affecting the host cells.

\section{S16-20}

ANALYSIS OF THE EUTATIVE ACTIVE SITE OF HEPATITIS A VIRUS (HAV) 3C PROIEASE BY SITE-DIRECIED MUTACENESIS

Dollermaier, G., Cassinotti, P. and Weitz, M., Institute
for Clinical Microbiology and Immology, 9000 for Clinical Microl

HAV is a member of the picomaviridae. Its RNA genome may be divided into 3 coding regions (P1, $P 2$ and $P 3)$. The $P 3$

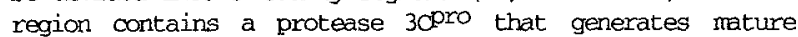
proteins by cis-cleavage of the initial polyprotein precursor. Catalytic activity of 3 pro has been demonstrated in a vacciniavirus/T7 RNA-polymerase hybrid expression system. Computer aided aligments of HAV 3 Cpro sequence with that of other picomaviruses imply that amino acid residues His -44 and/or His-191, Asp-98 and Cys-172 are camponents of the 3 pro catalytic site. To test this hypothesis appropriate P3 plasmid recombinants were constructed for site-directed mutagenesis and production of vacciniavinus recombinants. The following 30 pro mutant plasmids were obtained: C172G, C172S, D98N, H44Y. Furthermore, a vacciniavirus recombinant was produced containing the mutant HAV P3 (C172G) insert. This recambinant virus is currently used in expression experiments to test whether the proteolytic activity of $30^{\text {pro }}$ is inhibited.
S16-21

HEPATITIS A VIRUS (HAV) NONSTRUCIURAL PROTEIN $2 B$.

Gosert, R., Cassinotti, P. and Weitz, M., Institute for Clinical Microbiology and Irmunology, CH-9000 St. Gallen

$\mathrm{HAV}$ is a unique member (Hepatovinus) of picornaviridae. Computer-aided comparison of its RNA genome to those of other picornavinuses lead to a predicted structure of the HAV polyprotein. Most of the putative nonstructural proteins have so far not been physically identified. To shed light on the structure of the predicted polyprotein P2 region a vaccina virus/T7-hybrid recombinant was procuced This expressed in $T 7$ polymerase expressing animal cells (BT7-H) the complete HAV open reading frame. HAV specific expression products were identified by radioimmoprecipitation and by SDS-PAGE. The analysis with an antibody specific for putative nonstructural protein $2 \mathrm{~B}$ revealed a 27,5 $\mathrm{kDa}$ peptide. This is in contrast to its predicted size (12 $\mathrm{kDA}$. However, aralysis with anti-(putative) $2 \mathrm{~A}$ antiserum confirmed an upstream location of the 2B N-terminus. The new 2B peptide was also identified in lysates of HAV infected MRC-5 cells. Hence, recombinantly expressed HAV polyprotein underwent authentic processing and the predicted P2 organization has to be modified.

\section{S16-22}

A UNIQUE MECHANISM IN PRIMARY PROTEOLYTIC CLFAVAGE OF THE HEPATITIS A VIRUS (HAV) POLYPROTEIN.

Gosert, R., Weitz, M., Institute for Clinical Microbiology and Immunology, CH-9000 St. Gallen

HAV is a picornavinus. Its RNA genome regions P1-P2-P3 are translated into a giant polyprotein that is subsequently processed to mature proteins. In other picornaviruses primary cleavage occurs at distinct junctions either at $\mathrm{P} 1 / \mathrm{P} 2$ or at $2 \mathrm{~A} / 2 \mathrm{~B}$ (within $\mathrm{P} 2$ ). The responsible activities are proteins $2 \mathrm{~A}$ or quasi enzymatically active peptides. To characterize proteolytic processing of the HAV polyprotein a vaccinia vinus/T7-hybrid recombinant (VP1-P2) coding only for P1-P2 region of HAV was used. Expression and analysis by radioimmunoprecipitation with specific antisera and SDSPAGE revealed that in absence of $P 3$ no processing of the P1-P2 peptide (160 kDa) occurred. Coinfection of cells with vP1-P2 and a virus recombinant expressing HAV protease $3 \mathrm{C}$ resulted in P1-P2 cleavage and identification of a new 27,5 $\mathrm{kDa}$ peptide (2B). Hence, $3 \mathrm{C}$ protease is sufficient for the primary cleavage of the polyprotein. The primary cleavage occurs between peptides $2 \mathrm{~A}$ and $2 \mathrm{~B}$. Transcleavage of this juntion by $3 \mathrm{C}$ protease is unique amoung the picornaviruses. 


\section{Neuronal Modelling: From Neurons to Networks}

\section{S17-01}

J.Götz (Preclinical Research,Sandoz Ltd,Basel), M. Goedert (MAC, Cambridge, UK)
A. Probst (Dept. of Pathology, Univ. Basel) \& K. Bürki (Precl. Hes.,Sandoz,Baself)

Tau/ERK2 Transgenic Mice as a Model for Alzheimer's Disease

We are interested in the development of animal models for one of the two major pathological changes found in the brains of patients with Alzheimer's Disease, the formation of neurotibrillary tangles, a maim component of which is abnormally phosphorylated Tau-protent. (Tau hiseh is a protein associated whic microtubufi.) Two undamental questions may be answered with our models. Fis on all we would wion. to reproduce the formalion or tangles, and secruty we hope lis an sw the question, of Senile Demertiz of the Alzheimer Type (SDAT.

At present there are only a few putative cancidate genes known which may be in At present there are only a few putative cancidate genes known which may be invalved in the hyperphosphorylation of taus in vivo, one of which is the MAP.-Binase approach and expressed the cDNA under the control of four ditterent promoters in transgenic mice. These promoters were tested for maximal expression. A similar approach was chosen to express the human Tau is isolom in the brain of transgenic mice. We have started to analyze different Tau and ERK2 expressing lines, Northem blot analysis has revealed high levels of expression for all transgenes (up to fivefold in comparison to endogenous (evels). The neuronal specificity of the transgenic ERK2expression was contirmed by in situ hybridization analysis. In addition several TAUexpressing lines were analyzed in Westernblots to determine the relative amount of $h^{\text {TTau }}{ }_{\text {ec }}$ protein in the brain. Sections of the brain were stained with Tau-specific antibodies to identity the neurons expressing Tau. From intercrosses of Tau- with ERK2-expressing lines, we have already identified double-postive mice which wo currently analyze by immunong

Whosphoryation status of Tau. 2 might develop tangles, whereas single transgenics might not.

\section{$\mathrm{S} 17-02$}

\section{Trisomy 16 Mouse Model for Alzheimer's Disease}

$B$. Ledermann and $K$. Bürki

Preclinical Research, Sandoz Pharma AG, CH-4002 Basel

Down syndrome (trisomy 21) results from an extra copy of human chromosome 21 (HSA 21). Included in the phenotype of this syndrome is a predisposition to develop the characteristic neurofibrillary tangles and neuritic plaques that are the neuropathological hallmarks of Alzheimer's disease (AD). Mice that are trisomic for chromosome 16 may provide a genetic model for studies relevant to clarify molecular mechanisms involved in $\mathrm{AD}$ as portions of mouse chromosome 16 are genetically homologous with HSA 21. Since Ts16 mice do not survive birth, chimaeras have been formed between Ts16 and normal ( $2 n$ ) embryos to rescue the Ts 16 cells for postnatal studies. In this study we describe the establishment of a mouse Ts16 embryonic stem cell (ESC) line that can serve as an unlimited source for chimaera generation via blastocyst injection of such ESC's. Male mice carrying Robertsonian translocations of chromosome 16 onto chromosome 11 and 17 were mated with wildtype female mice. ES cell lines from day 4 embryos were derived and one Ts16 ES cell line has been identified so far. Chimaeras were generated and the analysis of a first chimaera regarding the proportion of Ts 16 cells in the brain, demonstrated a signiticant contribution of the Ts16 cells to the brain tissue examined.

\section{S17-03}

\section{Modelling Action Potential Propagation through EMBryonic DoRsal Root Ganglon Cells.}

CHR. LOSCHER, R.QUADRONI*, J. STREIT \& H.-R, LOSCHER, DEPT. OF PHYSIOLOGY, UNIVERSITY OF BERN, $\&$ " NNST. OF THEORETICAL PHYSICS, ETHZ, ZURICH

i. Impulse conduction in axons has integrative functions. The dorsal root ganglion (DRG) of the embryonic rat put into culture forms a monolayer and is thus accessible to conventional microelectrode technique, which allows testing the above hypothesis. We have chosen a dual approach by allows testing the above hypothesis. We have chosen a dual approach by
correlating experimental electrophysiological data from the culture with correlating experimental electrophysiological data from
results obtained from a compartmental computer model.

results obtaned from a compartmental computer model.
ii. The safety factor for conduction was found to be low. Thus failutes of ii. The safety factor for conduction was found to be low. Thus failures of AP invasion of the DRG cell soma could occur at sites of impedance mismatch when a second stimulus felt into the relative refractory period
of the first or when the axon was repetitively stimulated. The electrotonic residues (ERs) of the failed APs had discrete amplitude levels, suggesting that the failures were always caused at the same site along the axon. These sites of low safety factor were found to be the branch poin in the unipolar DRG cell and the entrance of the stem piece into the soma in both cell types, the bipolar as well as the unipolar. A systematic comparison of both cell types showed that the AP conduction through the latter is more reliable. The length of this stem piece is inversely correlated to the delay caused at the branch point, as the electrical load of the soma is more efficiently masked by a long stem piece.

the soma is more efficiently masked by a long stem piece. geometric changes or at sites of only subtle impedance mismatch when the membrane is shunted by open K-channels of a previous AP. A compartmental computer model based on vol tage clamp data from the literature reproduced the majority of the experimental findings. (SNF 31-27553.89 to H.-R.L.)
S17-04

EXCITATORY AND INHIBITORY SYNAPTIC CONNECTIONS
BETWEEN CELL PAIRS IN RAT HIPPOCAMPAL SLICE CULTURES.

Dominique DEBANNE, Beat H. GÄHWILER and Scott M. THOMPSON. Brain Research Institute, August Forel-Strasse 1 CH-8029 ZURICH

Pairs of individual neurons were recorded with sharp electrodes or in whole-cell configuration, in hippocampal slice cultures of rat. Synaptic connections were studied between pre- and postsynaptic cells in CA3 and CAl hippocampal fields by eliciting single action potential in the presynaptic cell. Monosynaptic excitatory connections were highly probable between $\mathrm{CA} 3$ and $\mathrm{CAl}$ pyramidal cells $(p=0.76, n=82)$ with almost no feed-forward inhibition $(p=0.01)$. By contrast, disynaptic IPSPs, blocked by CNQX or bicuculline were observed with a very high probability $(\mathrm{p}=0.6)$ between $\mathrm{CA} 3$ pyramidal cells. Monosynaptic excitation was found in $56 \%$ of cases between CA3 neurons $(n=43)$ but only in $27 \%$ of cases $(n=11)$ between two CAI cells. Monosynaptic IPSPs with very short latencies $(<\mathrm{Ims})$ and blocked by bicuculline, but not by $\mathrm{CNQX}$, were recorded within area CA3 (5/6) and within CA1 (2/3), but not between CA3 and CAl fields $(2 / 2)$. Probability of transmitter release, studied in recordings where failures could be unambiguously distinguished from spontaneous or smail PSPs, was found to be close to $100 \%$ at both excitatory and inhibitory synapses. Specific inhibitors of presynaptic release at excitatory and inhibitory terminals (i.e. adenosine and opioid peptides, respectively) gradually reduced the amplitude of the PSPs indicating that the release at excitatory and inhibitory unitary synapses is multiquantal.

\section{S17-05}

A CRITICAL TIME PERIOD FOR GROWTH OF NEURITES ACROSS A LESION IN NEONATAL OPOSSUM SPINAL CORD IN CULTURE.

Z. Varga.S. Erulkar and J. Nicholls*, Dept. Pharmacology, Biocenter, University of Basel, 4056 Basel, Switzerland

The CNS of a neonatal opossum, Monodelphis domestica, shows profuse growth of fibers across a lesion. As in other mammals, such repair is not seen in adults. Experiments have been made to define the age of the animal and stage of development at which repair ceases to occur. The entire CNS was removed from animals aged 3-6 days or 11-14 days after bith. The spina! cord was crushed and growth of fibers assessed 5 days later by electrical recording of impulses conducted through the crush. Repair after lesions to spinal cords of younger animals occurred in $38 \%$ of the trials ( 45 animals, aged 3-6 days). In nervous systems from older animals (11-14 days after birth) the success rate was low (only 4 out of 38 preparations). Similarly, the carbocyanine dye Dil labeled numerous tibers in spinal cords of younger animals (12 out of 21), whereas only one preparation out of 15 showed scant outgrowth into lesions in the older animals. Our results suggest that there is a critical period for neuronal repair; at about 12 days nerve fibers lose the ability to grow across lesions. It is an advantage that at this later stage the nervous system is still small enough to survive in vitro. Features correlated with these changes are oligodendrocyte differentiation and myelin formation. With immunofluorescent staining as well as electron microscopy myelination becomes apparent at 8 days after birth. We are now using time lapse-videomicroscopy to analyze molecular mechanisms that could play a part in promoting or preventing repair.

Supported by Grants to J.G.N. from the Swiss Nationalionds (31-3626292) and from the Internat. Res. Inst. for Paraplegia.

\section{S17-06}

DIRECT MEASUREMENTS OF ACTIVITY IN THE DENDRITES OF SPINAL CORD NEURONS USING VOLTAGE-SENSITIVE AND CALCIUM DYES. M.E. Larkum, M.G. Rioult, H-R. Lüscher. Physiologisches Institut, University of Berne. CH-3012 Berne, Switzerland.

The dendrites of motoneurons (and subsequently many other neurons) have been assumed to be electrically passive since the work of Coombs, Eccles and Fatt in the 1950's. However, direct evidence has been impossible to come by until recently with the advent of appropriate dyes and equipment for measuring very small and very fast optical signals. We examined the propagation of action potentials, both spontaneous and evoked, in the dendritic trees of spinal cord neurons using a $12 \times 12$ array of photodiodes and a CCD camera as well as conventional microelectrode techniques. Organotypic slice cultures of embryonic rat spinal cord were stained with a voltage-sensitive dye (di-8-ANEPPS) or a calcium dye (Fluo-3) and recordings were made from motoneurons identified by their location and morphology.

The results indicate that there is in fact an increase in calcium in the dendrites that accompanies action potentials whether synaptically evoked or evoked by current injection at the soma. Spikes in the dendrites up to $160 \mu \mathrm{m}$ from the soma are much larger than could be explained by models assuming passive dendritic trees. Work is currently underway to test the possibility that sodium conductances might contribute to the propagation of action potentials in the dendrites.

Supported by Swiss National Science Foundation (Grant no. 31-37553.89) 
S17-07

AUDITORY AXONS IN THE CAT CORPUS CALLOSUM

S. Clarke, V. Bajo-Lorenzana, E. Rouiller, F. de Ribaupierre, R. Kraftsik. Instituts of Physiology \& Anatomy, University of Lausanne, Switzerland.

Cat auditory cortex contains multiple cochlear representations in both hemispheres that are interconnected through the corpus callosum (CC). The distribution of auditory callosal axons was studied on the mediosagittal level after injections of biocytin at electrophysiologically identified locations. Single injections were done in AI (2 cats), $A A F, A I I$ and $P A F$; multiple injections in $A I$ \& $A A F$ in one cat. An additional cat received 1 injection in SII. The mediosagittal image of CC was subdivided into 30 segments along a rostro-caudal axis. Labelled axons crossing the midline were counted in coronal or horizontal sections. Axons from auditory fields were found in the posterior $2 / 3$ and those from the somatosensory cortex in the anterior third of CC. Axons from a discrete injection (ca $1 \mathrm{~mm}$ in diameter) spread over as much as $1 / 5$ of $\mathrm{CC}$. The rostrocaudal distribution of axons at the midline reflected roughly the rostrocaudal position of the field of origin, but apparently not the tonotopic order of a given field.

\section{S17-08}

FUNCTION OF THE MULTIMERIC TUBULIN-BINDING DOMAIN OF MICROTUBULE-ASSOCIATED PROTEIN 2 (MAP2). K. Ashbridge, B. Weisshaar, B. Ludin and A. Matus, Friedrich Miescher-Institut, P.O.Box 2543, 4002, Basel.

The neuronal microtubule-associated protein MAP2 binds to microtubules via a domain containing 3 or 4 repeats of an 18 amino acid sequence. We have previously shown that when cultured nonneuronal cells are transfected with MAP2 their microtubules become stiffer and are then capable of supporting process outgrowth when their cortical actin cytoskeleton is depolymerised. We hypothesise that this stiffening effect of MAP2 is caused by the 18 amino acid sequences binding to neighbouring tubulin subunits in the walls of the microtubules thus reducing their freedom of movement relative to one another. To investigate this further we have created MAF2 mutants in which 1 or 2 repeats of the tubulin-binding motif were deleted. These were tested by transfection and expression in cultured cells. The results confirm that the number of repeats affects the stability, stiffness and the cytoplasmic arrangement of cellular microtubules.

\section{S17-09}

ASTROCYTES AND MICROGLIAL CELLS DURING XENOPUS OPTIC NERVE REGENERATION. Alliod, C. and Rungger-Brändle, E. Microscopie électronique, Clinique d'Ophtalmologie, HCUG, 1211 Genève 14

In contrast to the amphibian optic nerve (ON), ON of adult mammals is unable to regenerate after injury. Astrocytes disappear from and macrophages accumulate at the lesioned site (Blaugrund et al., $J$. Neurocytol. 118, 105, 1992). We have followed the distribution of astrocytes and microglial cells in Xenopus tadpole ONs over $10 \mathrm{~d}$ after mechanical crush. Astrocytes were stained for cytokeratin and vimentin, and microglial cells for Griffonia Isolectin B4. Soon after crush, immunoreactivity for intermediate filament proteins was strongly increased. Astrocytic processes extended into the lesion from both proximal and distal stump. At $\mathbf{5}$ d, astrocytes had crossed the injured region. In the distal stump, some of them contained vacuoles indicating phagocytic activity. While in the normal oN microglial cells were ramified and scarce, $2.5 \mathrm{~d}$ after crush they were roundish and vacuolized, and scarce within the lesion but numerous distal to it. At $10 \mathrm{~d}$, their number was decreased and most of them had reacquired ramifications.

Conclusively, in the tadpole ON, astrocytes are virtually never absent from the lesion site but rather extend rapidly into it. Astrocytes may thus provide a guiding support for outgrowing axons. Microglial ceils that are frequent in the distal stump, do not accumulate at the lesion. The behaviour of the two cell types differs profoundly from that in mammals and is likely to be one of the factors that may contribute to successful neuronal regeneration of the amphibian ON.
$517-10$

SYNCHRONOUS ACTIVITY IN SUBSTANTIA NIGRA PARS RETICULATA OF THE ALBINO RAT. Alessandro E.P. Villa* Victoria M. Bajo Lorenzana Institut de Physiologie, UNIL, CH 1005 Lausanne.

The major efferent projections of substantia nigra pars reticulata (SNR) convey information to the thalamus, to the tegmentum, to the superior colliculus, and to the spinal cord. Such a range of targets suggests a highly organized activity within SNR and this study investigates the temporal organization of spike trains. In the portion of SNR between +2.7 and $+4.5 \mathrm{~mm}$ interaural levels, we recorded 40 single units along 6 electrode tracks in 4 young female Wistar rats. Most units ( $n=32$ ) fired in a non-regular Poisson process, although only a small number $(7 / 32)$ was characterized by an average burst size with more than 3 spikes. Crosscorrelograms were computed for 25 pairs of units simultaneously recorded from the same electrode and for 26 pairs simultaneously recorded from distinct electrodes. Most crosscorrelograms (27/51) showed a symmetrical peak centered on time zero, which indicates a tendency to synchronous firing. When units were recorded from the same electrode tip we observed that $1 / 8$ to $1 / 40$ of their spikes were synchronous. About half of the significant interactions were characterized by a relatively long duration ( $>250 \mathrm{~ms}$ ) and are likely to correspond to a different mechanism of synchronization. These results support the hypothesis that SNR cells receive common afferences of two different types and their fine temporally organized activity yields coordinated activity between the target structures.

\section{S17-11}

\section{CHARACTERIZATION OF KININ-LIKE IMMUNOREACTIVE} Kaelin A.*, Gerber-Bologa L. \& Sprumont P.: Institut d'anatomie et
d'embryologie spéciale de l'Université, CH-1700 Fribourg (*present address Neurophysiology Lab, Dept. Ophtalmology, University Hospital, CH-8091 Zürich)

We were previously able to demonstrate the presence of kinin-like immunoreactive structures in the mouse brain. In order to characterize them, we have now performed a double-immunofluorescence study using both we have now performed a double-immunofuorescence sti-n using both polyclonal anti-bradykinin (BK) and mouse midened to be a specific neuronal marker.

After fixation with Bouin-Hollande solution, the brains were removed, embedded in taraffin and serially sectioned at $10 \mu \mathrm{m}$. The sections were incubated with the antibodics, which were visualized by an indirect immunofluorescence technigue using FITC for NSE and by an avidin-biotin technique using Texas Red for BK.

Double-immunofluorescent cells were found in the thalamus ventralis, in the nucleus cochlearis ventralis and in the nucleus mesencephalicus nervi trigemin (V). BK-positive cells in the nucleus principalis V were NSE-negative in spite of their typical neuronal aspect the other BK-positive structures ependyma tanicytes) also were always NSE-negative. Most of the NSEpositive BK-negative cells were seen in the cortex. The neuronal nature of many kinin-like immunorcactive structures in the mouse brain is thus ascertained.

\section{\$17-12}

ORIENTED LIGHT-INDUCED PATTERNING OF MINIMAL PEPTIDIC SEQUENCES ONTO BIOCOMPATIBLE SURFACES TO CONTROL NEURONAL CELL ATTACHMENT AND DIFFERENTIATION

Clémence, J. F.1, Ranieri, J. P. ${ }^{2}$, Aebischer, P. ${ }^{2}$, and Sigrist, H. ${ }^{1}$, Institute of Biochemistry, University of Berne, $\mathrm{CH}-3012$ Berne; 2Division Autonome de Recherche Chirurgicale, CHUV, $\mathrm{CH}-1011$ Lausanne.

Small peptidic sequences of laminin (YIGSR, IKVAV) are known to promote attachment and/or neurite outgrowth of various neuronal cell lines (PC12, NB2, NG108-15). New and established heterobifunctional photo crosslinkers were used to immobilize these peptides in a topically defined fashion onto biocompatible surfaces such as hydroxylated fluorinated ethylene propylene (FEP-OH) and poly(vinylaicohol) (PVA). N-[m[(3-trifluoromethyl)diazirine-3-yl]phenyl]4-maleimido-butyramide (MAD) was thermochemically linked to the synthetic peptide CDPGYIGSR via its $\mathrm{N}$-terminal cysteine, leaving the bioactive part (YIGSR) free for cell receptor interaction. MADCDPGYIGSR was radioactively labeled by reductive methylation and purified by reversed phase HPLC. Patterned ( 20 and $300 \mu \mathrm{m})$ peptide immobilization was attained by photocoupling onto PVA and FEP-OH and visualised by autoradiography. Light-structured biomaterial surfaces with molecularly oriented nerve growth promoting peptides were investigated for spatially controlled neuronal cell attachment and differentiation. 
$S 17-13$

\section{THE EFFECT OF CORTICOSTERONE (B) ON THE LIMBIC SYSTEM OF LEAN RATS}

Doyle P., Guillaume-Gentil c., RohnerJeanrenaud F., Jeanrenaud B. Laboratoires de Recherches Métaboliques, Geneva, Switzerland Local cerebral glucose utilization (LCGU) of control and $B$-treated rats was investigated. Discrete areas of the B-treated rat brains had both increases and decreases in LCGU. These areas were associated with the limbic cortex (hippocampus, septum, some thalamic and hypothalamic nuclei). These regions are interconnected and have reportedly inhibitory or excitatory effects within the limbic region. It is striking to observe that these inhibitory or stimulatory effects were corroborated by Binduced decrease or increase in 2-deoxyglucose uptake, respectively. This gives $B$ a putative role in the limbic regulation.

S17-14

MOLECULAR MECHANISMS OF BRAIN DEVELOPMENT IN DROSOPHILA.

S. Therianos, G. Boyan, H. Reichert. Institute of Zoology, University of Basel.

To initiate a molecular genetic analysis of brain development in insects, we are characterizing the mechanisms of embryonic brain development in the fly Drosophila. Farly in embryogenesis, a small number of molecularly diverse brain neuroblasts generates the neurons of the brain. Subsequently, pioneering brain neurons initiate axonal outgrowth along glialbound brain compartments. These pioneering neurons establish the primary brain tracts. The pioneering brain axons express the cell-cell adhesion molecule fasciclin I dynamically during outgrowth and initial fasciculation; a subset of the pioneering axons expresses fasciclin II. The axonal framework of the embryonic brain tracts is used for outgrowth and fasciculation by subsequently differentiating axons and, thus, prefigures the tracts of the mature brain. A comparison of early brain development in Drosophila and in vertebrates reveals common mechanisms for brain development. Supported by the Swiss NSF.

\section{S17-15}

Cervical primary afferent input to vestibulo-spinal neurones projecting to the dorsal horn: A double labelling study in the rat.

S. Bankoul; Institute of Anatomy, University of Fribourg, CH.1700

Wheat germ agglutinin-horseradish peroxidase (WGA-HRP) was injected by pressure into cervical spinal ganglia $\mathrm{C} 2$ or $\mathrm{C} 3$. In the same experiments Fluorogold (FG) was iontophoretically injected into the ipsilateral dorsal horn of different cervical spinal cord segments.

Anterogradely labelled fibers (WGA-HRP) were present mainly in the external cuneate nucleus, but also to a lesser extent in the caudal part of the medial and descending vestibular nuclei (MVN, DVN).

The FG injections, restricted to the cervical dorsal horn, revealed retrogradely labelled cells in the central part of the caudal MVN.

A double exposure (fluorescent and polarisation optics) under the microscope showed primary afferent fibers surrounding like baskets retrogradely labelled FG cells. The projection from cervical ganglia cells to the MVN represents a pathway for direct afferent information from neck receptors (in particular muscles) to vestibular nuclei and supplies the MVN with afferent information which could enable the vestibulospinal neurones, projecting to the dorsal horn, to influence the local information processing.
$517-16$

RELATIONSHIP OF OLIGODENDROCYTE PROCESSES TO PARVALBUMIN-CONTAINING AXONS

E. WERUAGA, I. BLÜMCKE, P. EGGLI*, S. KASAS, M.R. CELIO Institut d'Histologie et d'Embryologie générale, Université $\mathrm{CH}-1700$ Frihourg. *Anafomisches Institut, Universifät CH-3012 Bern.

The sheet-like processes of oligodendrocytes wrap themselves spirally around central axons, thus forming the myelin sheath. A single oligodendrocyte may donate internodal segments of myelin to each of $20-30$ or more adjacent axons. It is not known, if one oligodendrocyte picks out axons randomly or if it prefers axons with a certain diameter of with a certain chemical specificity. We have studied this last possibility by combining intracellular injection of a fluorochrome, and immunohistological detection of calciu ing proteins $\left(\mathrm{CaBP}^{\prime} \mathrm{s}\right.$ ), markers for the axons of certain subpopulations of nerve cells. Lucifer Yellow was injected into oligodendrocytes of the optic nerve, of living rat brain slices. The oligodendrocytes were identified by their electrophysiological properties. Slices containing the injected cells were immunostained for parvalbumin or calretinin using second antibodies labelled with Texas red. Using confocal laser-scanning microscopy combined with a three-dimenslonal reconstruction programm, the relationship between oligodendrocyte processes and axons positive for one of the CaBP's was determined. For ultrastructural confrmation, single, Lucier-Yellow injected, oligodendrocytes were photoconverted, and the CaBs s-positive axons were revealed by gold-labejled antibocies. The few oligodendrocytes injected show the classic morphology, that is of a small cell body and few processes runging parallel to the course of the axons. The proximity between glial processes and positive axons can be easily appreciated in confocal laser-scanning images. A preferential association of oligodendrocyte processes with parvalbumin-positive axons has not as yet been found.

Thus, the confirmation of the hypothesis that oligodendrocytes choose their axonal targets according to chemical cues awaits further work including the injection of a larger number of these cells.

S17-17

CALRETININ IS EXPRESSED IN CAJAL-RETZIUS CELLS IN ORGANOTYPIC CULTURES OF THE DEVELOPING RAT NEOCORTEX

D.M. Vogt Weisenhorn, E. Weruaga Prieto, L. Blumcke, M.R. Celio

Institute of Histology, University of Fribourg, Pérolles, $\mathrm{CH}-1700$ Fribourg

Calretinin (CR), an EF-hand $\mathrm{Ca}^{2}+$-binding protein, is expressed in Cajal-Retzius cells during development of the rat cerebral cortex. These cells are located in the marginal zone and are the earliest cortical neurons to be generated. They have been considered as unusual on account of their morphology and their fate during corticogenesis. The functional significance of Cajal-Retzius cells and their destiny in adulthood is still controversial. In order to approach these questions we have applied organotypic culturing methods.

Slices of neocortex from brains of 0-6 days-old rat pups were cultured for 10-21 days applying the interphase technique (Stoppini et al., J. Neurosci. Methods 37 1991) or the roller-tube technique (Gähwiler, J. Neurosci. Methods 4, 1981). The fixed cultures were immunolabelled with an antibody against CR.

The obtained results showed that CR positive structures develop in vitro like in vivo. However, in organotypic cuitures their distribution corresponded to a more vivo. However, in organotypic cuitures their distribution corresponded to a more mature stage than the situation on the day of the explantations. Many CR-positive cells were seen in layer I. They were horizontally oriented or had a pyriform shape. Based on their morphology these cells were identified as Cajal-Retzius cellis. CRpositive cells were numerous in layer II-III and showed mostly a bipolar morphology. Some mulipolar cells could also be observed. A fine net of CRpositive fibers and puncla was tound in layers $l$ and $N$. The demonstration that Cajal-Retzius cels are deteciable in these cultures will allow us to follow the fate of these cells dynamically and intervene in their function by applying exogenous
factors.

S17-18

SNAP-25 IN TEE PERIPHERAL NERVOUS SYSTEM OE RATS AND DURING SCIATIC NERVE REGENERATION Duc C., Institut d'Histologie et d'Embryologie, Rue du Bugnon 9, 1005 Lausanne

SNAP-25 is a membrane-associated protein that is involved in axonal elongation and synaptic formation in the central nervous system. We have studied the expression of SNAP-25 in the peripheral nervous system (PNS) by using a polyclonal antibody (a gift from S. Catsicas, Glaxo Institute for Molecular Biology, Geneva). SNAP-25-immunoreactivity (IR) was observed in motor and sensory neurons of the PNS. In the cell bodies of DRG neurons, SNAP-25-IR was found in a perinuclear network probably corresponding to the Golgi apparatus. In the sciatic nerve, all myelinated and non-myelinated axons were SNAP-IR. In peripheral tissues, all nerve endings were positive. The preterminal schwann cells of motor axons were also SNAP-25-IR. After sciatic nerve section, many myelinating schwann cells also expressed SNAP-25-IR.

In conclusion, SNAP-25 is expressed by neurons in the PNS. It seems to be also expressed by preterminal schwann cells and by myelinating Schwann cells during regeneration. 
S17-19

REPAIR OF COMPLETELY TRANSECTED SPINAL CORD OF NEONATAL OPOSSUM IN CULTURE

H.A. Vischer, Dept. Pharmacology, Biocenter, Uni. of Basel, Switzerland Woodward et al. (J. exp. Biol. 1993, 1993, 176, 77-88) have shown that fibers grow rapidly and profusely across a lesion made by crushing the spinal cord of the neonatal opossum Monodeiphis domestica. In such experiments careful controls must be made to establish that fibers were in fact broken by the lesion. Tests have now been made to determine whether similar repair can occur after a more drastic lesion involving complete transection of the cord. After dissection of the entire CNS from animals aged 3-8 days, the cord was cut into two with scissors at the $\mathrm{C} 5-\mathrm{C} 7$ level. The two ends were glued together with matrigel on a sylgard mold, which encompassed and held the CNS. The rostral end was labeled with the fluorescent carbocyanine dye Dil in order to visualize growing fibers. In 33 preparations fibers grew over the cut into the separated part of the spinal cord. Such growth could extend to $500 \mu \mathrm{m}$. Fibers grew straight, branched and multidirectionally, or in fascicles. In another set of experiments cords were combined from different animals of the same or different ages. Again, fibers could grow across the cut but they did so less frequently. These results set the stage for investigating fiber growth after rotating the two ends at a defined angle and for analyzing factors that influence the direction of growth

Supported by grants to JGN. from the Swiss National Fond (31-36262) and the international Research Institute for Paraplegia.

\section{$517-20$}

Differential distribution of MAP 1a and aldolase in mouse cerebellum

F. Touri, R. Hawkes. ${ }^{*}$, B. M. Riederer

Institute of Anatomy, University of Lausanne, 1005 Lausanne, *Department of Anatomy and Neuroscience Research Group, University of Calgary, Canada

MAP 1a is a microtubule associated protein of $360 \mathrm{KD}$. In rat brain two monoclonal antibodies, $A$ and $B W 6$, recognized MAP 1a in neurons and their axonal and dendritic processes. In mouse cerebellum, monoclonal A recognized Purkinje cells and granule cells uniformly, as already described for rat brain. In contrast, monoclonal BW6 stained selectively some dendrites of Purkinje cells, forming bands in the molecular layer. We compared this striation with that obtained with a monoclonal antibody, anti-zebrin II, which recognized aldolase $C$. In the mouse vermis, zebrin stained in a striated pattern, but complementary to BW6. These results demonstrate that MAP la is present in forms which are differentially distributed in the mouse cerebellum. These observations may be explained either by differences in metabolic states of neurons, or by differences in their regional functions, or by differences in the regional stability of microtubules. This work was supported by grant No 31-33447.92 from the Swiss National Science Foundation.

\section{S17-21}

\section{SYNAPTOGENESIS IN AGGREGATING BRAIN CELL \\ CULTURES.}

Pithon E. ${ }^{1}$, Riederer B.M. ${ }^{2}$, and Honegger P.

IInstitut de Physiologie. Université de Lausanne. 1005 Lausanne

Institut d'Anatomie. Université de Lausanne. 1005 Lausanne

Serum free aggregating brain cell cultures prepared from 16 day old fetal rat telencephalon are used as a model to study brain development. In these cultures it is possible to distinguish ontogenetic events such as cell proliferation, differentiation and myelination. In the present study we examined synaptogenesis by analyzing the expression of synaptic proteins such as synaptophysin, SNAP-25, synapsin I, and brain spectrin. Synaptosomal fractions isolated from aggregate cultures at different developmental stages were analyzed by western blotting. Immunoreactivity for synaptic proteins was detectable already at day 12 in culture, suggesting that the first synapses are already present at this early stage. The expression of synaptic proteins strongly increased between day 20 and day 30 in culture, reflecting a period of intense synaptogenesis. Treatment of the culture with an elevated concentration of $\mathrm{KCl}(30 \mathrm{mM})$ increased the expression of synaptic proteins, suggesting a stimulation of synaptogenesis. These findings demonstrate the utility of this approach to study brain synaptogenesis, and possibly synaptic plasticity.

\section{S17-22}

TOPOGRAPHY OF OPTIC FIBERS IN THE ADULT TREB SHREW (TUPAIA BELANGBRI)

Drenhaus, U., Gunten, A.V., Rager, G.; Institute of Anatomy, University of $\mathrm{CH}-1700$ Ereiburg The retina of Tupaia comprises three types of ganglion cells (RGCs) analogous to $\mathrm{X}-, \mathrm{Y}-$, and $W$-cells found in other mammals. These celis differ in size and in their distribution pattern: large RGCs are frequent in temporal, small RGCs in nasal retina.

As the fiber diameter correlates with the size of its respective RGC, it can serve as a parameter for the topographic representation of RGCs in the nerve. To study this we analyzed the optic nerves of three Tupaia. Using the electron microscope we recorded the density, diameter, and the position of fibers in the nerves.

We found, that fibers with the largest diameter are located dorso-temporally and close to the center of the nerve. They are surrounded by zones of smaller fibers. The diameter decreases gradually towards the periphery and is smallest in the ventro-nasal region of the nerve.

Since the fiber diameter distribution corresponds to that of the size of RGC-types in the retina, we assume that the topographic relationships in the nerve and the retina are similar.

(supported by Swiss NF grant 31-36468.92)

\section{S17-23}

DEVELOPMENT OF AN IN VTTRO BLOOD-BRAIN BARRIER MODEL BY INTERFACING A CO-CULTURE OF ENDOTHELIAL CELLS AND NERVOUS ORGANOTYPIC CULTURES

Stoppini Luc, S. Duport, L. Parist, C. Oropesa., Departement of Pharmacology, CMU, 1211 Geneva 4

The micro environment of the central nervous system is important for neuronal function. In this context, the blood-brain (BBB) provides and maintains the extracellular medium that is compatible with normal neuronal and synaptic activity. Due to the difficulties inherent using whole animal as an experimental model to study permeabillty and metabolic processes at the cellular level, major efforts have been engaged in recent years, in order to bring up suitable in vitro models. We are presently developping an in vitro BBB by interfacing a coculture of endothelial cells monolayers and nervous organotypic cultures. The main idea of this study is based on the premise that the complex intercellular interactions between the different cell types pertaining to the nervous tissue and the neighbouring endothelial cell is of fundamental importance to promote a realistic BBB system. We expect that organotypic culture of nervous tissue, combined to endothelial cell monolayers, will initlate and maintain a BBB phenomena in vitro. We will test more specifically the hypothesis that neuronal activities (spontaneous or elicited) will influence glial cell response which will in turn modify endothelial properties. Preliminary results clearely show a good nervous tissue and endothelial cell survival. Tight junction like structures could be identified using freezefracture or conventional transmission electron microscopic thechniques. (Work supported by Chemodyne Genève ).

\section{S17-24}

\section{A NEW OLIGODENDROCYTE SPECIFIC GENE IS EXPRESSED} DURING LATE MYELINATION

A. Holz, N. Schaeren-Wiemers, U. Pott, R. J. Colello and M. E. Schwab, Brain Research Institute, University of Zurich, AugustForel-Str.1, CH-8029 Zurich

The process of myelination is a prerequisite for the proper function of the brain since it enables rapid saltatory conduction of axons. In the central nervous system (CNS) myelination is performed by oligodendrocytes. A differential screening approach was used to isolate new rat CDNA clones that are expressed in this glial cell type. Here we report the further characterization of two of these novel cDNAs which appear to be expressed specifically in oligodendrocytes.

The two characterized CDNA clones (tentatively called CNS1 and COP-25.1) share an approximately 420 bp region of sequence identity which suggests that they are derived from the same gene by alternative splicing. Within this area a putative open reading frame is located. We demonstrated by Northern blot analysis and in situ hybridization that the two mRNAs of the novel gene are expressed exclusively in oligodendrocytes. However, the mRNAs show a different specific spatial localization within the cell. We compared MRNA expression of myelin basic protein (MBP) and proteolipid protein (PLP), with that of CNS1 and COP-25.1 in brain and optic nerve during development. It appeared that the mRNAs of the nove gene were delayed significantly as compared to MBP and PLP mRNAs. 
THE ROLE OF DEPRESSION FOR RHYTHMOGENESIS IN AN EXCITATORY SPINAL NETWORK

J.Streit. Physiologisches Institut, Bühlplatz 5, 3012 Bern

In organotypic slice cultures of the embryonic spinal cord, rhythmic spontaneous activity arises when the inhibitory synaptic transmission is blocked. The rhythmic activity consists of bursts of regular oscillations of activity at $4-5 \mathrm{~Hz}$. This study investigates the origin of the regular oscillations. When stimulated electrically at $5 \mathrm{~Hz}$, the synaptic transmission in the cultures showed strong depression by about $50 \%$ on average. The synaptic depression was highly variable among individual connections and tended do be less pronounced in frequently used connections.

When random depression ratios with an average of $50 \%$ at $5 \mathrm{~Hz}$ were implemented in a computer model consisting of 100 randomly connected excitatory cells, regular oscillations of activity did not arise, even in the presence of synchronous pacemaker cells. However, when individual depression ratios were adapted according to the rate of activity of individual connections, regular oscillations at $4-5 \mathrm{~Hz}$ arose in the presence of few unsynchronized pacemakers. This finding suggests that the regular oscillations in the cultures originate in a network formed by the plasticity of synaptic depression. Supported by SNF and Roche Foundation
CORRELATION BETWEEN THE ONTOGENY OF DOPAMINE $\mathrm{D}_{1}$ RECEPTOR BINDING AND IRNA

Maier A., Schlumpf M., Beer H.F., Schubiger P.A. and Lichtensteiger $W$., Inst. of Pharmacology, Univ. of Zurich, CH-8057 Zurich and Paul Scherrer Inst., Radiopharmacy Div., CH-5232 Villigen PSI

The ontogeny of expression of Dopamine $D_{1}$ receptor mRNA in the rat brain and binding to the receptor was studied at 12 time points from gestational day (GD) 13 to postnatal day (PN) 60 by quantitative receotor autoradiography and in situ hybridization.Long Evans rat pups and fetuses of time pregnant rats were frozen and sectioned coronally and sagitally at $10 \mathrm{um}$. $D_{1}$ receptor binding, determined with the selective 1 I I 125 I-SCH 23982 was first noted on GD 18 in the developing striatum, basal ganglia and the olfactory tubercle, reaching adult levels at around PN 14. The expression of $D_{1}$ receptor mRNA was studied by using a mixture of 3 specific ${ }^{35} \mathrm{~S}$-labelled oligonucleotides. On GD 13 messages were noted in the developing stiatum, olfactory tubercle and retina. This study shows a good regional correlation between the development of receptor binding and mRNA, however, mRNA precedes detectable binding to the receptor by several days.

\section{RNA in Recognition and Catalysis}

S18-01

SITES ON QB PLUS AND MINUS STRAND RNA INVOLVED IN TEMPLATE RECOGNITIONBY QB REPLCASE

Daniel Schuppli, Imma Barrera, José M. Sogo+ and Hans Weber Institut für Molekularbiologie I, Universität, 8093 Zürich, and +Institut für Zellbiologie, ETH-Hönggerberg, 8093 Zürich

Earlier work had shown that $Q \beta$ replicase forms complexes with $Q \beta$ plus strand RNA via interactions at two internal binding sites, the $S$ - and the M-site, while binding of the $3^{\prime}$-end is mediated by host factor. In contrast, the 3 'end of the minus strand appeared to be directly accessible by replicase. We have prepared a series of plus and minus strand variant RNAs containing either internal deletions or terminal deletions extending from the 5 '-end. The template activities of the variants were determined by single-round replication assays. For the plus strand we find that while deletion of the S-site remains without effect (as expected from previous resulss), deletion of the M-site reduces template activity to $25 \%$ or less compared to wild-type RNA; the residual activity shows a decreased dependence on the presence of host factor. The results agree with an important role of the M-site interaction both with replicase and with host factor for the formation of productive initiation complexes. The template activity of the minus strand was unexpectedly found to be strongly dependent on the presence of a segment between $\mathrm{nf} 76$ and 210 from the 5 -end. This shows that recognition of the minus strand by replicase does not only involve interactions near the 3 -end but requires a previously unknown structural feature near the $5^{\prime}$-end of this template.

S18-02

\section{CHARACTERISATION AND PURIFICATION OF THE MAMMALIAN SPLICING FACTOR 3B}

Karsten Gröning and Angela Krämer

Département de Biologie Cellulaire, Université de Genève, 30, quai Ernest-Ansermet, 1211 Genève 4

Splicing of nuclear pre-mRNAs takes place within multicomponent complexes (spliceosomes) that are assembled by interactions between the pre-mRNA, snRNPs and protein splicing factors. We have purified two splicing factors that function in the formation of the pre-splicing complex. SF3a consist of three subunits of 60,66 and $120 \mathrm{kDa}$ and corresponds to three proteins present in the functional 17S but not in the 12S U2 snRNP. It binds to the 125 U2 snRNP only in the presence of SF3b, suggesting a two step assembly pathway of $17 S$ U2snRNP: binding of SF3b to the $12 S$ U2 snRNP generates a $15 \mathrm{~S}$ intermediate particle that is converted to the active 17S U2 snRNP by the subsequent association of $\mathrm{SF} 3 \mathrm{a}$. Comparison of proteins enriched in the SF3b fraction with polypeptides found in the purified $15 S$ U2 snRNP suggests that SF3b comprises at least five of the other $17 S$ U2 snRNP specific proteins and together with SF3a promotes the U2 SnRNP/premRNA interaction. By cDNA cloning, two of the subunits of SF3a have been identified as homologs of well characterized yeast proteins that function at the same stage of spliceosome assembly in yeast.

\section{S18.03}

\section{CDNA CLONING OF MAMMALIAN SPLICING FACTOR SF1}

Silke Backes, Graeme Bilbe* and Angela Krämer

Département de Biologie Cellulaire, Université de Genève, 30 , quai Ernest-Ansermet, 1211 Genève; 'Ciba-Geigy AG, 4002 Basel

Splicing factor SF1, a heat-stable 75-kD protein, functions early during spliceosome assembly. Tryptic peptides of SF1 were sequenced and CDNA libraries were screened with degenerate oligonucleotides. The information obtained by comparing the sequences of three overlapping cDNAs suggests the existence of at least three mRNAs that could be derived from a common premRNA by alternative splicing. In agreement with this assumption mRNAs of the expected sizes that are differentially expressed depending on cell line or tissue are detected by Northern blotting. in theory, three polypeptides could be generated that differ by the presence or absence of 7 internal amino acids and in their Ctermini. The deduced amino acid sequence is rich in prolines and contains several possible phosphorylation sites and a putative leucine zipper. Proline-rich domains, which are also present in splicing factors PSF and SAP62, as well as leucine zippers have been found in transcription factors and could mediale protein/protein contacts, suggesting that SFI could function during splicing by interaction with other spliceosomal proteins.

\section{S $18-04$}

\section{CHARACTERIZATION AND PARTIAL PURIFICATION OF} SPLICING FACTOR SF4

Patric Grüter and Angela Krämer

Département de Biologie Cellulaire, Université de Genève, 30 , quai Ernest-Ansermet, 1211 Genève

Pre-mRNA splicing is catalyzed by a multicomponent complex (the spliceosome) that consists of small nuclear ribonucleoprotein particles (snRNPs) and non-snRNP protein factors. The spliceosome is assembled in a stepwise fashion on the pre-mRNA. Chromatographic fractionation of HeLa cell nuclear extracts and subsequent reconstitution of splicing in vitro has allowed the separation and isolation of SnRNPs and several protein factors. SF4 triggers the transition between splicing complex $\mathrm{B}$, which contains all spliceosomal snRNPs but is not competent for catalysis, and complex $\mathrm{C}$, the active spliceosome. This transition involves a conformational change that leads to altered base pairing interactions between snRNAs and pre-mRNA. The purification of SF4 has been impeded by its low abundance; however, a correlation between SF4 activity and a polypeptide of $50 \mathrm{kD}$ could be established in the most purified fractions. We are currently investigating whether this protein represents SF4. Interestingly an ATP-dependent RNA-helicase cofractionates with SF4 through several purification steps. Whether or not this activity is relevant for the splicing process is under investigation. 
S18-05

SELECTION OF IRON-RESPONSIVE EIEMENT RNAS WITH HIGH AFFINITY FOR IRON REGULATORY FACTOR

Henderson, B.R., Menotti, E., Bonnard, C., and Kühn, L.C., ISREC, CH-1066 Epalinges

Iron regulatory factor (IRF) post-transcriptionally requlates iron homeostasis via binding to mRNA iron-responsive elements (IREs). The IRE loop sequence $\left(5^{\prime}-C_{A G U G N} 3^{\prime}\right)$ and "bulge" cytosine are phylogenetically conserved. We prepared a pool of 16,384 IRE molecules randomized at these 7 nucleotide positions, and employed in vitro selection to identify optimal sequences which bind human IRF. We define two major classes of high affinity RNA ligand, the optimal loop sequences of each are 5'-CAGUGN-3' (wild-type) and $5^{\prime}$-UAGUAN-3'. This novel finding predicts base-pairing within the loop between positions 1 and 5. All nucleotide substitutions in the "bulge" or at loop positions 2,3 and 4 decreased binding by 368 to 998 . In addition, binding specificity of rat IRF differed with that of the related rat IRE-binding protein, IRF. In vitro analysis of IRE-Iike stem-loops identified by computer search has not yet revealed any new IRE-containing genes.

\section{\$18-06}

ASSEMBLY OF U7 SnRNPs

B. Stefanovic, I. Haussmann, R. Lührmann ${ }^{t}$ and D. Schümperli, Abtl. f. Entwicklungsbiologie, Zoologisches Institut Bern, Baltzerstr. 4, Bern and ${ }^{1}$ IMT, Philipps-Universität, 35037 Marburg, Germany.

In Xenopus laevis oocytes, wild type mouse U7 RNA gets assembled into functional U7 snRNPs, both when transcribed from an injected gene or when injected as in vitro synthesized RNA. If the Sm binding site of U7 RNA is converted into the canonical Sm sequence (U7 Sm OPT), the RNA assembles into a particle which accumulates more efficiently in the nucleus but which is nonfunctional. This U7 Sm OPT particle inhibits the function of preformed endogenous U7 snRNPs, most likely by nonproductive binding to histone pre-mRNAs. Histone pre-mRNA processing can be demonstrated in cis if U7 RNA is placed ' ${ }^{\prime}$ ' of histone pre-mRNA and injected into oocytes. Only U7 Sm WT sequences are active in this cis processing. Three proteins can be photoaffinity crosslinked to U7 Sm WT RNA, the common snRNP proteins $G$ and $B / B^{\prime}$ and a $U 7$-specific protein of $40 \mathrm{kD}$ ( $50 \mathrm{kD}$ in mouse). These crosslinks map to closely spaced positions within the Sm binding site with the U7-specific crosslink being located most 3'. U7 Sm OPT RNA does not become crosslinked to the U7 specific protein and is also more tightly associated with Sm proteins. We now investigate the in vitro assembly of U7 snRNPs using these characterized U7 RNAs and a preparation of highly enriched SnRNP proteins. U7 Sm WT and U7 Sm OPT RNAs associate with common Sm proteins in this system, but the interaction with the U7-specific protein could not be demonstrated.

\section{S18-07}

\section{PURIFICATION AND CHARACTERIZATION OF THE DOUBLE-STRANDED RNA MODIFYING ENZYME FROM CALF THYMUS.}

Mary A. O' Connell and Walter Keller, Biozentrum, University of Basel, $\mathrm{CH}-4056$ Basel.

The double-stranded (ds)RNA modifying enzyme, which can convert adenine to inosine, was first identified in Xenopus laevis, but has since then been detected in different species and in mammalian cells. The enzyme is a specific adenine deaminase which uses dsRNA as substrate and recently, it has been postulated to be responsible for a specific mRNA editing reaction. We have purified this enzyme to homogeneity, approximately 400,000 fold from calf thymus whole cell extracts. The protein was purified primarily by ion exchange chromatography over six columns, with the final step being chromatography on a dsRNA affinity column. The purified protein has a molecular weight of $115 \mathrm{kD}$ and is the only protein present when enzymatically active fractions are visualized on an SDS-polyacrylamide gel stained with silver. The dsRNA modifying enzyme is a very low abundant protein. We are in the process of further characterizing the purified enzyme and of cloning cDNAs coding for it.

\section{S18-08}

3' Processing of histone pre-mRNAs: A phylogenetic comparison Reto Kohler, Petra Duda and Daniel Schümperli. Zoologisches Institut der Universtitä Bern, Abteilung für Entwicklungsbiologie, Baltzerstr. 4, CH-3012 Bern, Switzerland. We are using a phylogenetic approach to study the biochemical reaction by which animal histone pre-mRNAs are processed at their $3^{\prime}$ ends. In mammals, the efficiency and specificity of this reaction is known to depend absolutely on the U7 SnRNA which interacts with conserved spacer sequences downstream of the processing site. A highly conserved hairpin element which precedes the cleavage site serves as a binding site for an additional processing factor, but its importance for efficient processing varies greatly between different histone genes and between extracts of different mammalian cell lines. To determine whether the relative importance of the hairpin and space elements have been conserved during evolution, we analysed the processing of histone genes from three different animal phyla in vitro, i.e. vertebrates (mouse), arthropods (Drosophila melanogaster) and nematodes ( $C$. elegans). In the mouse system, processing was strongly dependent on the presence of a mouse spacer element. In nuclear extracts of Drosophila Kc cells, processing occurred exclusively when a Drosophila spacer element was present in the RNA. Processing efficiency was not reduced by foreign (mouse, C. elegans) hairpins. In whole cell extracts of Ascaris lumbricoides embryos, an inverted situation was observed. $3^{*}$ processing products were only produced by RNAs that included an upstream segment derived from a $C$. elegans histone gene, irrespective of the spacer sequences present. These surprising results correlate with certain unique features in the $C$. elegans upstream element. We are currently in the process of cloning Ascaris lumbricoldes histone genes, which will allow us to verify our results in a homologous system.

\section{S18-09}

STABILIZATION OF THE CHLOROPLAST PSOD IRRA IN CHLAMYDOMONAS RETNHARDTII

Nickelsen, J., van Dillewyn, J, and Rochaix, J.-D.; Dept. Molec. Biol., University of Geneva, 1211 Geneva.

The nuclear photosynthetic mutant nac2-26 of chlamydomonas reinhardtii is specifically affected in the stability of the psbD mRNA encoding the D2 protein of the photosystem II reaction center. A chimeric message containing the psbD leader region is destabilized in the nac226 nuclear background in vivo indicating that this region plays an essential role for RNA stability. In vitro studies revealed that the psbD leader is degraded by endonucleolytic cleavages in the mutant. The psbD leader-specific RNA binding activity of a $47 \mathrm{kDa}$ protein was detected that is missing in nac2-26.

\section{S18-10}

Detailed mutational analysis of histone RNA $3^{\prime}$ end formation

Carmen Spycher, André Furger, Adrian Streit, Daniel Albrecht, D. Schümperli, Zoologisches Institut der Universtität Bern, Abteilung für Entwicklungsbiologie, Baltzerstr. 4, CH-3012 Bern, Switzerland

Histone RNA $3^{4}$ ends are formed by endonucleolytic cleavage resulting in poly(A)mRNAs with a highly conserved 3'-terminal hairpin loop structure. For the mouse H4-12 gene major and minor processing sites are located 3 and 5 nucleotides downstream of the hairpin, respectively. For the cleavage reaction, the U7 snRNP interacts with the spacer element of histone pre-mRNA by base-pairing through the $5^{\prime}$ end of its RNA moiety, U7 RNA. We have observed that this base-pairing potential extends further than previously recognised in either direction. We have made site-directed mutagenesis in this potential hybrid region and around the processing site. RNA processing and complex formation with the U7 snRNP were analysed by incubation in nuclear extract from mouse $\mathrm{K} 21$ cells. Our results indicate that base-pairing with U7 RNA both in the classical spacer element and downstream of it are very important for histone RNA processing. In contrast, sequences upstream of the spacer element do not seem to be involved in base-pairing with U7 RNA, but mutations in this region may affect processing by other mechanisms. Systematic mutations of the highly conserved four nucleotides immediately following the hairpin show no qualitative or quantitative effects in the processing reaction. Alteration of nucleotides 5 to 7 around the minor processing site, however, result in a dramatic decrease in processing efficiency and alter the specificity of the cleavage site. In further experiments we will introduce specific nucleotide modifications at the two processing sites, which should allow us to characterize potential cleavage factors by chemical crosslinking. 
MUTAGENESIS APPROACH TO IDENTIFY AMINO ACIDS INVOLVED IN RNA-BINDING OF IRF.

Kaldy, P., Peitsch, M. and Kïhn, I. C.

ISREC, $\mathrm{CH}-1066$ Epalinges.

Iron Requlatory Factor (IRF) binds specifically to an RNA-stemloop (Iron Responsive Element; IRE) present in ferritin and transferrin receptor mRNAs, and is involved in their posttranscriptional regulation. IRF is an apoprotein which is converted to the cytoplasmic aconitase by insertion of an [Fe-S]-cluster, constituting the active site. This inhibits the RNA-binding properties. To determine which amino acids are responsible for RNA-binding, we made sitedirected mutagenesis of residues i) supposed to be close to the active site ii) conserved between the IRF's of different species and iii) absent from bacterial or mitochondrial aconitases which do not bind IREs. We used the known crystal structure of mitochondrial. aconitase to select nine surface-exposed regions for mutagenesis. One of the mutants had a decreased RNA-binding but normal aconitase activity. Within this region, two basic residues are important for the binding.

\section{Developmental Genetics}

S19-01

\section{DETECTION AND ANALYSIS OF PAX-6 HOMOLOGUES IN EVOLUTION}

Callaerts P., Glardon S., Loosli F., Quiring R. and Gehring W.

Cell Biology, Biozentrum, Basel.

The $P a x$ genes encode a family of DNA binding factors which share the paired-box and play an important role in the control of development. They can be subdivided into four different classes which differ in the presence or absence of other conserved sequence motifs coding for a paired type homeobox or an octapeptide. The $P a x-6$ gene, which contains a paired-box and a homeobox, has been cloned from humans, mice, rats and zebrafish. Recently, we have cloned the $P a x-6$ homologue of Drosophila melanogaster. The five genes share a very high degree of sequence identity both in the paired-domain and the homeodomain. Similar to its mammalian counterparts, the Drosophila gene is expressed in the eye primordia and the nervous system. In addition, mutations in $P a x-6$ affect eye development: Aniridia in humans, Small eye in mouse and rat, and probably eyeless in Drosophila. This would imply that the $P a x-6$ homologues share a conserved function in eye morphogenesis in both vertebrates and invertebrates. Furthermore, this suggests that $P$ ax- 6 may have been present in a common ancestor. Therefore, we are now trying to isolate $P a x-6$ homologues from other, more primitive organisms by means of the polymerase chain reaction, and to determine whether they are implicated in eye development. Putative candidates have been identified from a number of species and are being characterized. Their sequence similarity and some evolutionary implications will be discussed.

\section{S19-02}

\section{PAX7: MEMBER OF THE MYOGENIC REGULATORY}

\section{NETWORK?}

Michele Bernasconi, Stefan Mühlebach*, Jean-Claude Perriard* and Beat W. Schäfer, Dept. of Pediatrics, University of Zürich and *Institute of Cell Biology, ETH Zürich, Switzerland.

In search for novel regulators potentially involved in myogenesis, we identified a homeobox of the paired type in human muscle. Subsequent cDNA cioning revealed that we had cloned the full length coding region of human PAX7. Our human sequence extends the known mouse cDNA both on the $5^{+}$and the $3^{\prime}$ end. As a first step in order to test for a functional role of PAX7 in myogenesis, we analyzed its expression pattern during chicken development. Transcripts are present in the neural tube and in the dermamyotome of the developing somites. Therefore, the pattern of expression of Pax7 is conserved between mouse and chicken. Next, we assessed the expression of $\mathrm{Pax} 7$ in different mouse and human cell lines. We found $\mathrm{Pax} 7$ transcripts specifically in myogenic cells and not in any other cell types. Interestingly, $\operatorname{Pax} 7$ is already present at the myoblast stage. Moreover, 10T1/2 fibroblasts converted to myoblasts by either transfection of myoD or treatment with 5-azacytidine expressed Pax7. whereas the parental cells did not. While myoD was able to activate Pax7 in 10T1/2 cells, expression of Pax7 was not sufficient to induce the myogenic phenotype. Nevertheless, our expression data are consistent with a role of $\mathrm{Pax} 7$ during the mesodermal commitment to the myogenic lineage.

\author{
S19-03
}

\section{TARGET GENES OF ANTENNAPEDIA}

Keegan Liam and Gehring Walter J, Biozentrum, Universiry of Basel, Klingelbergstr. 70, CH-4056 Basel, Switzerland

Using an enhancer trap screen we have identified two genes that may be direct targets of Antennapedia involved in forming the adult leg. spalt major is repressed in the leg imaginal disc and teashirt is activated by Antennapedia. We wish to determine whether the control of these genes by Antennapedia is direct. spalt major is expressed in a ring in the antennal disc and is repressed by Antennapedia in the leg disc. The antennal ring is repressed by ectopic Antennapedia expression that transforms the antenna to a leg. We are defining an enhancer at spalt major that is required for antennal ring expression and repression by Antennapedia. We are using various approaches to show that Antennapedia binds to the antennal ring enhancer at spalt major. These include polytene chromosome banding studies using antibodies to Antennapedia as well as in vitro DNA-binding and genetic experiments.

S19-04

\section{THE ROLE OF EMPTY SPIRACLES IN DROSOPHILA HEAD DEVELOPMENT}

Walldorf, Uwe and Gehring, Walter J.,Dept. of Cell Biology, Biozentrum, University of Basel, Klingelbergstr. 70, CH-4056 Basel

The segmental organization of the Drosophila head is achieved by a flow of positional information from maternal gene products to the zygotic gap genes. Recent genetic analysis has identified three new gap genes involved in head development. One of these genes is the empty spiracles (ems) gene. Mutations in ems cause severe defects in the head and the Filzkörper at the posterior end are missing. The ems gene encodes a putative transcription factor with a homeodomain. The ems RNA is expressed in two phases of embryonic development. First expression is seen at the blastoderm stage in a single anterior band, correlating with its function as an anterior gap gene. Later during embryogenesis ems is expressed in the posterior spiracles as well as lateral regions of each segment where the tracheal pits form and lateral neuroblasts originate. Using B-gal fusions we could identify at least five different regulatory elements in the ems promotor region responsible for tissue specific expression of the gene. A 300 bp element responsible for the early expression which is dependent on the maternal gene bicoid, the key gene of the anterior system, and the terminal gene tailless was identified and studied in detail. This element contains two bicoid and two tailless binding sites. The effects of muations in these binding sites on ems expression will be discussed.This analysis should allow us to elucidate the molecular mechanisms by which the morphogen bicoid regulates subordinate target genes like ems in the Drosophila embryo. 
S19-05

\section{ANALYSIS OF A PAX -6 HOMOLOGUE FROM \\ DROSOPHILA}

Walldorf Uwe, Quiring Rebecca, Kloter Urs and Gehring Walter.J.

Dept. of Cell Biology, Biozentrum, University of Basel,

Klingelbergstr.70, $\mathrm{CH}-4056$ Basel, Switzerland

The $P a x-6$ gene of the mouse encodes a transcription factor with both a paired-and a homeodomain. $P a x-6$ is affected by several allelic mutations in the Small eye (Sey) gene. Sey mutations cause a reduction of the eyes in heterozygotes, and homozygotes lack both eyes and nasal openings and die as newborns. The corresponding mutation Aniridia in humans affects eye and craniofacial development in a similar manner. We have isolated a Pax-6 homolog from Drosophila (DPax-6), which shares more than $90 \%$ sequence identity in the paired-and the homeodomain with the mammalian genes. Also outside of these domains we find considerable sequence conservation arguing for a true $P a x-6$ orthologue. The Drosophila gene spans $\sim 16 \mathrm{~kb}$ and is expressed in the brain and the ventral nervous system of the embryo, and in the eye imaginal discs and the brain of the larva. The gene was mapped to the eyeless (ey) locus. Mutations at the ey locus cause either the partial or complete absence of the compound eyes or embryonic lethality. In one ey allele we have identified a transposable element in the first intron of $D P a x-6$, which affects $D P a x-6$ transcription in the eye discs, suggesting that $D P a x-6$ is encoded by the $e y$ gene. This implies that $P a x-6$ (Sey) in the mouse is homologous to $e y$ in Drosophila. Thus, despite of the different modes of eye morphogenesis, the same gene seems to control eye development in insects and vertebrates.

\section{S19-06}

The Drosophila SRF homolog is expressed in tracheal cells and maps within a genomic region required for tracheal development Markus Affolter, Jacques Montagne, Uwe Walldorf, Jay Groppe, Urs Kloter, Marilina LaRosa and Walter J. Gehring

Dept. of Cell Biology, Biozentrum, University of Basel, CH-4056 Basel

The Drosophila homolog of the vertebrate serum response factor (SRF) was isolated by low stringency-hybridization. Nucleotide sequence analysis revealed that the Drosophila SRF homolog (DSRF) codes for a protein which displays $93 \%$ of sequence identity with human SRF in the MADS domain, the region required for DNA binding, dimerization and interaction with accessory factors. The DSRF gene is expressed during several phases of embryonic development. Both the RNA and the protein are maternally provided to the egg and slowly decrease in their levels during gastrulation. After germ band retraction, high levels of zygotic expression were observed in a distinct subset of peripheral tracheal cells distributed throughout the embryo. Many of these cells are at the tip of tracheal branches and are in direct contact with the target tissues these branches tracheate. The DSRF gene was mapped to position $60 \mathrm{C}$ on the second chromosome, and overlapping deficiencies which remove the gene were identified. Analysis of tracheal development in embryos carrying these deletions revealed a degeneration of most of the major branches of the tracheal system. Although the initial migration of tracheal cells was not affected in those deficiency embryos, many tracheal cells appeared not to maintain their formerly correct position and continued to migrate. Thus, the DSRF gene might play a role in the proper formation and maintenance of the major branches of the trachea.

\section{S19-07}

\section{ISOLATION OF POXN MUTANTS IN DROSOPHILA}

K. Brcic-Kostic, E. Frei, and M. Noll

Institut für Molekularbiologie II,Universität Zürich, 8057 Zürich

The poxn gene plays an important role during Drosophila neurogenesis. It is expressed in specific subsets of sensory mother cells (SMCs) and neuroblasts. The SMCs that express poxn differentiate into polyinnervated external sense (p-es) organs. In the absence of poxn. SMCs differentiate into mono-innervated rather than poly-innervated external sense organs. As the poxn gene contains a paired-box, it probably encodes a transcription factor. Since the function of poxn in the central nervous system is not known and no poxn mutants are available, an EMS mutagenesis screen was initiated to isolate such mutants.

Based on the assumption that poxn null alleles are lethal, we screened for lethals uncovered by the deficiency $D f(2 R) W M G$, which includes poxn, but survive over the deficiencies $D f(2 R) X T E-18$ and $D f(2 R) K L-32$ flanking poxn. In a further test, such lethals are screened for the absence of embryonic p-es organs.

If poxn mutants are not lethal, they will escape this screen. Therefore, we are inducing local hopping of P-elements that have inserted in the vicinity of poxn. The offspring of flies that display an altered eye phenotype are screened for P-element insertion into poxn.

We hope that these approaches will generate mutants that will be crucial for future studies of poxn function in neurogenesis.
S19-08

\section{FUNCTIONAL DISSECTION OF THE DROSOPHILA PAIRED-DOMAIN AND HOMEODOMAIN PROTEIN GOOSEBERRY}

Xuelin Li, Lei Xue, and Markus Noll

Institut für Molekularbiologie II, Universität Zürich, 8057 Zürich

In the Drosophila genome, three genes, gooseberry ( $g s b)$, gooseberry neuro ( $g s b n)$, and paired ( $p r d)$, that perform distinct developmental functions encode transcription factors with highly conserved paired- and homeodomains in their $\mathrm{N}$-terminal halves. Recently, we showed that these proteins retain the same functional potential despite their apparently unrelated $C$-terminal halves. We are now focusing on the question of what part of these proteins are required for their functions. To this end, the following experiments are being carried out.

1. Rescue of prd mutants by the $g s b$ coding region under the control of the prd regulatory region.

2. Rescue of $g s b$ and prd mutants by the coding region of their mammalian homolog, Pax-3, under the control of the gsb or prd regulatory region.

3 . Rescue of $g s b$ and $p r d$ mutants by the $g s b$ coding region, from which either paired- or homeobox, or both have been deleted, under the control of the $g s b$ or prd regulatory region.

Our experiments would be expected to provide insight into such problems as the evolution and function of transcription factors with multiple DNA binding domains. Ideally, we would be able to mimic "gene splitting" which occurred during evolution.

\section{Orphan receptor COUP-TF II during neurogenesis}

Beat Iutzl, Shigeru Kuratani1, Austin Cooney ${ }^{2}$, Ming Tsai2 and Gregor Eichele ${ }^{1}$. Department of ${ }^{1} \mathrm{Biochem}$. and ${ }^{2} \mathrm{Cell}$ Biology, Baylor College of Medicine, Houston, TX 77030, USA

Members of the steroid/thyroid hormone receptor superfamily are involved in the control of cell identity and of pattern formation during embryonic development. Studies in tissue culture cells and bandshift experiments revealed that chicken ovalbumin upstream promotertranscription factors (COUP-TFs) can act as modulators of various steroid/thyroid hormone receptor pathways. To begin to study the role of COUP-TFS during embryogenesis, we cloned a chicken COUP-TF (CCOUP-TF II). Analysis of CCOUP-TF expression by in situ hybridization revealed high levels of CCOUP-TF II mRNA in different regions of the developing CNS raising the possibility that CCOUP-TF II is involved various aspects of neurogenesis. Our studies are focused on the development of spinal motor neurons and of the diencephalic neuromeres. 


\section{Gene Expression and Function}

\section{\$20-01}

Chromatin structures and transcription of rDNA in yeast Saccharomyces cerevisiae

Reinhard Dammann, Renzo Lucchini, Theo Koller and José $M$. Sogo; Institute of Cell Biology, ETH-Honggerberg, CH-8093 Zürich

The chromatin structure of yeast ribosomal DNA was analyzed in vivo by cross-linking intact cells with psoralen. We found that in exponentially growing cultures the regions coding for the $35 S$ rRNA precursor fall into two distinct classes. One class was highly accessible to psoralen and associated with nascent RNAs, characteristic for transcriptionally active rRNA genes devoid of nucleosomes, whereas the other class showed a cross-linking pattern indistinguishable from that of bulk chromatin and was interpreted to represent the inactive rRNA gene copies. By cross-linking the same strain growing in complex or minimal medium, we have shown that yeast cells can modulate the proportion of active (non-nucleosomal) and inactive (nucleosomal) rRNA gene copies in response to variations in environmental conditions which suggests that yeast can regulate rRNA synthesis by varying the number of active gene copies, in contrast to the vertebrate cells studied so far. Whereas intergenic spacers flanking inactive rRNA gene copies are packaged in a regular nucleosomal array, spacers flanking active genes show an unusual cross-linking pattern suggesting a complex interaction of regulatory factors and histones with DNA.

\section{$\mathrm{S} 20-02$}

Transcriptional regulators and triplet repeats in mammals and yeast

Patrick Rigoni, Sandro Rusconi, Institut f. Molekularbiologie II der Universitüt Zïrich, Winterthurerstr. 190,8057 Zïrich, Switzerland.

Trinucleotide repeats are often found in the coding sequence of transcriptional regulators in both mammals and yeast. However, their function is yet unclear and data collected in our laboratory on the glucocorticoid receptor even suggest that they may not have any, and that their presence is probably the result of a selfish replicative behavior. Nonetheless, we believe that these motifs can be used as tags to identify flexible protein domains typical of modular proteins such as transcription factors. Another kind or repeat (coding for a poly-glutamine/alanine) has been discovered in the yeast regulators GAL11 and SSN6. From Northern blots, we know that such $C A G G C N$ repeats are present also in higher eukaryotes and we want to isolate them by constructing an enriched cDNA library using a $5^{\prime} \mathrm{RACE}$ protocol. So far, no mammalian protein bearing the motif Gln/Ala has been characterized. Among the positive clones we hope to find the homologous or paralogous of GAL11 and SSN6 that have escaped so far conventional cloning techniques. Meanwhile, we have been testing the effect of coexpressed yeast GAL11 on different reporter-activator combinations in mammalian cells. Preliminary results show that Gall1 but not the mutant form Gal11P can inhibit the activation properties of some (not all) Gal4 chimeras. We are also producing antibodies directed against oligo-Ala and oligo-Gln and oligo-Gin/Ala motifs in order to better characterize natural proteins containing these repeats.

520.03

\section{ANTERO-POSTERIOR AXIS IN C. ELEGANS}

C. Wittmann, K. Bnunschwig, T. R. Bürglin*, H. Tobler and F, Muller Institute of zoology, University of Fribourg, Pérolles, Switzerland *Department of molecular biology, MGH, Boston MA, USA le-rail: Wittramactrani52.bitnet

We are interested in the early development of $C$. elegans, particularly in the contribution of genes whose homologues in vertebrates and $D$. melanogaster mediate positional informations during development. Therefore, we are working on ceh-13 which belongs to a cluster of at least 4 homeobox containing genes. This cluster is considered to be equivalent to the homeotic cluster of Drosophila and to the Hox clusters in vertebrates.

By generating $\beta$-galactosidase transgenic lines, we have observed that ceh-13 is expressed very early during embryogenesis. Embryonic expression appears to be restricted to the posterior half of the embryo, which is rather surprising, since the ceh-13 homologues in Drosophila and vertebrates are anterior-specific. During larval stages, $c e h-13$ is expressed in neuronal cells. These results are now in the process of being confirmed by immolocalization of the ceh-13 protein product with a polyclonal antiserum.

In order to clarify the function of ceh-13 in the c. elegans development, we have isolated a ceh-13 mutant From a Tcl insertion library, in collaboration with R. H. A. Plasterk from Amsterdam. From this worm, which looks WT, we are now trying to obtain a deletion derivative.
$\mathrm{S} 20-04$

ANIALYSIS OF A CYTOVILLIN-REIAATED ANTIGIN TRON ECEINOCOCCUS MULTILOCULARIS AND I. GRANULOSUS R. Felleisen \& B. Gottstein; Institute of Parasitology, University Bern, Bern, Switzerland Most patients suffering from alveolar echinococcosis ( $\mathrm{AE}$ ) respond to infection with a marked IgG synthesis directed against E.multilocularis metacestode antigen II/3, which represents a novel member of the family of cytovilin related proteins. Although the respective gene basically is also present and expressed in E.granulosus, most of the cystic echinococcosis (CE) patients do not recognize the antigen. This phenomenon was tackled by generating CDNA derived from full length II/3 genes from both Echinococcus species, performed by RT-PCR. Sequence analysis revealed a very high degree of conservation of the primary sequence (98.6\% homology). CDNA fragments were expressed as recombinant proteins and were comparatively assessed in ELISA respective to antibody-binding characteristics. Sera from patients suffering from $C E$ were showing no significant differences in reactivity with the antigens derived from both species. Therefore, parameters others than minor differences in the primary sequence seem to be responsible for the lack of antigen recognition with respect to $\mathrm{CE}$.

\section{S20-05}

Bovine herpesvirus 1 immediate-early protein BICPO is a zinc-dependen transactivator.

C. Fraefel, J. Zeng. Y. Choffat, M. Ackermann, and M. Schwyzer, Institute für Virologie. Blochemie und Zoologie der Universitüt Zürich, 8057 Zürich

Previous studies have shown that Bovine herpesvirus I (BHV-I) infected cell protein (BICP) 0 acts - depending on the promoter - as a strong transactivator or as a repressor in transient expression assays. The BICPO polypeptide contains a cysteine-rich zinc finger domain $\left(\mathrm{C}_{3} \mathrm{HC} 4 \mathrm{C}_{4}\right)$ which is conserved in a number of viral and cellular regulatory proteins including the BICPO homologs ICPO of herpes simplex virus type 1 and protein 61 of varicella zoster virus. This type of zine finger (the so-called RING finger) was shown to bind zinc ions but functional requirement for zinc has not yet been demonstrated, a gap which we aimed to fill with the present study. Transient expression assays were performed in oocytes which had been microinjected with thionein to chelate and deplete the intracelluiar pool of zinc. BKCPO-induced CAT activity of a promoter-CAT construct was 72 -fold higher than the basal CAT activity of the reporter plasmid. In the presence of thionein, BICPO-induced transactivation was reduced 5-fold. With a set of control experiments we excluded that thionein might affect franscription and protein synthesis in general.

To our knowledge this is the first demonstration of zinc-dependence for a member of the RING finger family.

\section{S20-06}

\section{Characterization of POU genes in zebrafish embryos}

Giselbert Hauptmann, Philipp Spaniol, Caroline Bornmann, Andreas Vogel and Thomas Gerster Abt. Zellbiologie, Biozentrum der Univ. Basel, 4056 Basel

We have identified by PCR and CDNA cloning five POU genes expressed during early embryogenesis of zebrafish. Four of these genes show extended homology to the $b r n-I$ class of POU genes. Preliminary evidence suggests that the $b r n-1$ like POU genes overlap with most of their expression domains. The gene studied in most detail so far (ZP-50) is first expressed on the ventral side of the future fore- and midbrain. Slightly later, expression is also found in rhombomeres 3 and 5 in the hindbrain. These thombomeres were identified by the specific expression of the Krox-20 marker using a novel in situ hybridization protocol which allows the simultaneous detection of two different transcripts using different color substrates. In the 24 hours old embryo a complex expression pattern is found involving a variety of brain structures and the spinal cord. The distribution of the transcript suggests that ZP-50 is mostly expressed in glia cells. Another POU gene we have identified (GP-9) defines a novel class of POU proteins. As a maternal mRNA it is initially ubiquitously present. After gastrulation its transcript is found most notably in the developing hindbrain in rhombomeres 2 and 4 for which so far no good molecular markers were known. We are investigating the roles the identified POU genes may have in zebrafish hindbrain segmentation.

We are currently attempting to manipulate gene expression in developing embryos by injection of RNA or by the production of transgenic fish. We are also screening the zebrafish genome for new developmental marker genes. Progress about these experiments will be presented. 
S20-07

Identification of Functional Elements and Reconstitution of the $\alpha 1(\mathrm{VI})$ Collagen Promoter

Willimann, $T$. and Trueb, B.

To gain insight into the regulatory mechanisms of collagen VI synthesis we have characterized the cis-acting elements of the chicken $\alpha 1(\mathrm{~V} \mid)$ collagen promoter. Footprinting experiments with nuclear extracts from chicken embryos revealed three distinct elements, designated $\mathrm{A}, \mathrm{B}$ and $\mathrm{C}$, that were protected from DNase I digestion. The nuclear proteins that interact with the three sites were identified by gel retardation assays in combination with the use of various oligonucleotide competitiors as well as specific antibodies raised against well-characterized transcription factors. Site $A$ was found to be a target for transcriptional activator AP1, whereas sites $B$ and $C$ were shown to be recognized each by two distinct nuclear proteins which belong to the $\mathrm{Sp} 1$ multigene family. To address the question whether the three sites alone are able to direct transcription, a minipromoter construct was created in which the sequences of sites $A, B$ and $C$ were placed in front of a reporter gene. After transfection into chicken fibroblasts, this construct exhibited a high relative promoter activity when compared to a large genomic fragment containing the basic $\alpha 1(\mathrm{VI})$ collagen promoter. Thus, the three sites are sufficient to induce transcription of this gene.

\section{$\mathrm{S} 20-08$}

MODULATION OF HORMONE-INDUCED AND BASAL LEVELS OF THE MOUSE MAMMARY TUMOR VIRUS PROMOTER

C. Cavin and E. Buetti, ISREC, 1066 Epalinges.

Two DNA regions upstream of the distal glucocorticoid receptor binding site interact with nuclear proteins that are tissue-specific (region a) or ubiquitous (region c). The characteristics of the DNA-protein interactions have been studied in vitro. These sequences, in different combinations with the natural MMTV promoter, were tested in transfection assays of fibroblast or mammary cell lines. We show that they are able to modulate the level of glucocorticoid-stimulated transcription.

The proximal region of the MMTV promoter has binding sites for the transcription factors CTF/NF-I and oct-1. Plasmids with a deletion or mutations in the oct-1 site were tested in stable transfections, that are most representative of the state of proviral DNA with respect to both number of integrated DNA templates and chromatin organization. We show that the oct-1 site is important for the basal level of promoter activity. The data further indicate that the functional outcome may depend both on the relative ratio of factors to DNA templates and on the relative affinity of binding sites, as determined by oligonucleotide competition footprinting.

\section{S20-09}

THE P48 DNA-BINDING SUBUNIT OF TRANSCRIPTION EACTOR PTF1 IS AN EXOCRINE PANCREAS-SPECIFIC bHLH PROTEIN A. Krapp, O. Hagenbiuchle and P.K. Wellauer, ISREC, 1066 Epalinges.

The pancreas-specific transcription factor PTF1 is a heterooligomer containing three distinct bHLH proteins. p75, which does not contact the DNA, is the rat counterpart of human bHLH protein 312 . This protein converts cytoplasmic into nuclear form of the factor in the amphibian oocyte assay. The p64 DNA-binding subunit is a bHL protein that is related to human transcription factor ITF2. The bHLH domain of p48 DNA-binding subunit shares extensive amino acid sequence similarity with that of developmental regulatory proteins from other organisms. p48 is specified by a mRNA that accumulates exclusively in the exocrine pancreas. This protein confers therefore, cell-specific DNAbinding to PTF1. p48 mRNA sequences are detected for the first time around day 16 of embryonic development. This is the earliest developmental stage at which PTEl binding activity and significant levels of mRNAs for exocrine pancreatic functions have been detected in the pancreas. The mRNAs that specify 048 before and after birth differ considerabiy in size. This observation suggests the possibility for the existence of a developmentally regulated switch in p48 gene promoter usage.
S20-10

CHARACTERIZATION OF THE N-OCT 3/BRN-2 GENE, A TRANSCRIPTION FACTOR EXPRESSED IN THE CENTRAL NERVOUS SYSTEM

Atanasoski, S., Solinas Toldo, S. ${ }^{1}$, Malipiero, U., Schreiber, E., Fries, R. ${ }^{1}$, and Fontana, A.

Division of Clinical Immunology, University Hospital, CH-8044 Zürich

Department of Animal Sciences, Federal Institute of Technology, ETH Zentrum, CH-8092 Zürich 1

Octamer transcription factors (Oct or OTF) are a subset of the POU family of transcription factors which regulate expression of cellular and viral genes by binding to the octamer sequence ATGCAAAT. Neurons and astroglial cells harbour, in addition to the ubiquitous Oct-1 factor, brain specific factors termed $N$-Oct $2,3,4$ and 5 . In the present study we determined the chromosomal localization of the gene encoding the N-Oct 3 transcription factor and characterized the structure of isolated $\mathrm{N}$-Oct 3 genomic clones. The chromosomal mapping was performed by analysis of somatic cell hybrid panels and radioactive and fluorescence in situ hybridization of human metaphase chromosomes. It is interesting that the $5^{\prime}$ end of the $\mathrm{N}$ Oct 3 coding sequence contains repetitive CAG and GGC residues. Several disorders have been discovered to be related to expansion of trinucleotide repeats. We are presently investigating if the N-Oct 3 CAG or GGC clusters are hot spots for such mutation mechanisms and if so, which diseases could be associated with it.

S20-11

CLONING AND ANALYSIS OF THE $C$. ELEGANS TELOMERES

Chantal Wicky, Heinz Tobler and Fritz Müller.

Institute of Zoology, University of Fribourg, Pérolles, CH-1700 Fribourg, Switzerland.

Besides the fact that telomeres represent specialised structures important for chromosome stability, the particular significance of cloned $C$. elegans telomeres is that they will contribute to the completion of the physical mapping of chromosomes and that they provide one set of elements for the constraction of artificial C. elegans chromosomes.

The cloning of $C$. elegans telomeres, however, is impeded by the fact that tandem repeats of the sequence TTAGGC are not only located at the ends of the $C$. elegans chromosomes, but also at many internal chromosomal sites. Therefore, we have developed a special protocol for the construction of a C. elegans endlibrary in $\lambda$ Zap.

The resulting telomeric library was screened for recombinants containing TTAGGC repeats and yielded about 70 positive clones. So far, 30 were analysed by partial sequencing and twelve of them satisfy all criteria required for telomeric clones. Their TTAGGC tandem repeats are located at the expected position, namely just adjacent to the blunt end cloning site in the polylinker. Furthermore, the G-rich strand of the repeats is oriented 5 ' to 3 ' towards the end of the fragment, thus corresponding exactly to the defined orientation of eukaryotic telomeric sequences.

Finally, hybridisation data with one of these putative telomeric clones show that a subtelomeric fragment hybridises to Bal31-sensitive fragments on a Southern blot with $C$. elegans DNA, indicating that this clone represents a C. elegans telomere.

$\mathrm{S} 20-12$

\section{TRANSCRIPTION THROUGH A YEAST ORIGIN OF REPLICATION ARS1 AFFECTS REPLICATION}

S.Tanaka, D. Halter, M.Livingstone-Zatchej, B.Reszel and F.Thoma, Institut für Zellbiologie, ETH-Hönggerberg, CH-8093 Zürich.

To investigate whether chromatin structure or transcription can interfere with replication, derivatives of the yeast TRP1ARS1 minichromosome were constructed that contain either the DED1 or PET56 promoter firing against the origin of replication ARS1. While the PET56 constructs transformed yeast at high frequencies and were maintained as high copy number minichromosomes, the DED1 constructs transformed at low frequency and the constructs were integrated in the genome suggesting that ARS function was impaired. Insertion of the H4-ARS, a second origin of replication, rescued a DED1 construct as a minichromosome (YRpDH3). The chromatin structure mapped at low and high resolution of the ARS1 region in $\mathrm{YRpDH} 3$ was indistinguishable to that of the PET56construct YRpFT61. Ananlysis of RNA showed that transcription was going through ARS1 in YRpDH3 and in YRpFT61, but the levels of transcripts in YRpDH3 were much higher. We conclude that transcription through ARS1 interferes with replication and prevents extrachromosomal maintenance. 
S20-13

DNA SEQUENCE OF A REPEATED DNA SEGMENT ON CIRCULAR AND LINEAR PIASMIDS OF BORRELIA BURGDORFERI

W.R. Zückert and J. Meyer, Abt. PZMOM,

Zahnărztliches Institut der Universität Basel

A plasmidassociated repeated DNA segment of B. burgdorferi strain B3I was cloned. At least two copies of this segment appear to be present on the $29 \mathrm{~kb}$ circular plasmid, whereas one copy is carried on the $50 \mathrm{~kb}$ linear plasmid of this strain. DNA sequence analysis revealed a region containing a novel 906 bp putative open reading frame (orfl) on the $50 \mathrm{~kb}$ linear plasmid. orf 1 displayed extensive sequence homology (958) to putative open reading frames present on the clones obtained from the $29 \mathrm{~kb}$ circular plasmid. Heterogeneity is mainly caused by 3 rd-base-wobbling. Flanking sequences share 85-958 homology. including the $5^{\prime}$, end of an additional putative open reading frame immediately downstream of orfl. Whether orfl and/or the related sequences are being transcribed and yield, in the case of orfl, an approximately $36.5 \mathrm{kD}$, Iysine-rich polypeptide, is yet unknown. The repeated sequence seems to be specific for $B$. burgdorferi sensu lato and therefore may be useful in nucleic acidbased diagnostics of Lyme disease.

\section{S20-14}

MOLECULAR BASIS OF DARK EYED ALBINTSM IN THE MOUSE Schmidt, A., Beermann, F. Swiss Institute for Experimental Cancer Research (ISREC), Ch. des Boveresses 155, CH-1066 Epalinges

Dark eyed albino $\left(c^{44 H}\right)$ is a recessive allele at the mouse aibino (c) locus, which encodes tyrosinase (monophenol, L-dopa:oxygen-oxidoreductase, EC 1.14.18.1), the key enzyme in melanin synthesis. Similar to type IB oculocutaneous albinism in humans, overall production of pigment is greatly reduced in dark eyed albino and only obvious in eyes. We have studied the molecular basis of the $c^{44 H}$ mutation and show that expression of the tyrosinase gene is not affected. After sequencing tyrosinase cDNA isolated from $\mathrm{C}^{44 \mathrm{H}} / \mathrm{C}^{44 \mathrm{H}}$ homozygotes we uncovered a single base alteration from wild type leading to a serine to isoleucine exchange. The importance of this mutation was demonstrated by generating transgenic mice containing a mutated tyrosinase minigene. This showed that the single base change is sufficient to severely depress pigment production in transgenic mice. We therefore conclude that the point mutation is responsible and sufficient to generate the dark eyed albino phenotype.

\section{S20-15}

\section{DOES INTRON DENSITY CORREIATE WITH THE} REPETITIVENESS OF CODING DNA?

Oliver Clay, Koichi Matsuo and Walter Schaffner Institut für Molekularbiologie II der Universität Zürich, Winterthurerstrasse 190, 8057 Zürich

Repetitive sequences in DNA can allow ectopic (outof-register) homologous recombination, which is often undesirable and can even lead to disease in humans. To prevent this, long homologies should often be interrupted during evolution. Accelerated mutation of repetitive sequences by so-called ripping may be one of the mechanisms used to accomplish this in fungi. We are investiqating if introns in vertebrate genes have an undiscovered role as interrupters of homology within and/or between genes, in addition to their established role in exon shuffling. By inserting homology-poor introns in an otherwise homology-rich region the genome could prevent ectopic homologous recombination. Such a mechanism could be especially useful where there is an advantage in encoding highly repetitive protein sequences. It appears that within the collagen gene family there is indeed a correlation between repetitiveness and intron density.
S20-16

TRANSCRIPTIONAI REPRESSION BY DNA METHYLATION Koichi Matsuo, John Silke, Oleg Georgiev and Walter Schaffner

Institut für Molekularbiologie II der Universität Zürich, winterthurerstrasse 190, 8057 Zürich

DNA methylation can inactivate promoter activity both by inhibiting binding of transcription factors and by stabilizing an inactive state of genes through methyl CPG binding proteins and/or tight chromatin structure. We have constructed reporter plasmids that are driven by LexA-VP16 fusion transcription factor. After methylation in vitro by CpG methylase, these constructs were transfected into Hela cells with the LexA fusion expression vector and the transcriptional activity was measured by SI mapping. A subset of CPG containing fragments (ca. $50 \mathrm{bp}$ ), when inserted between upstream LexA sites and the TATA box, repressed transcription in a methylation dependent manner. To further characterise this effect we are

investigating naturally occurring promoters with a similar $C p G$ configuration. Using genomic sequencing techniques, we are testing whether the activity from these promoters correlates with lack of methylation.

\section{S20-17}

\section{IDENTIFICATION OF NOVEL POU-DOMAIN GENES EXPRESSED DURING MOUSE MAMMARY GLAND DEVELOPMENT}

B. Jehn, G. Chicaiza, F. Martin and R.Jaggi

Universität Bern, AKEF, Tiefenaustr. 120, 3004 Bern

Members of the POU-family of transcription factors are involved in developmental and differentiation processes. Using a PCR-based cloning strategy with degenerated oligonucleotides we identified several POUgenes from the lactating and involuting mouse mammary gland. Three of these cDNA clones which contained as yet unknown sequences were chosen for further investigations: clone $\mathbf{5 . 8 0}$ which has a high homology to Pit- 1 , clone 5.54 which is related to the Brn-3 gene and clone $\mathbf{5 . 4 8}$ which belongs to the class III of POU-proteins. The expression levels were analyzed in different mouse organs and in several developmental stages of the mammary gland, including the postlactational process of involution. Because of the low abundance of the specific transcripts we used a semiquantitative, reverse transcriptase-mediated PCR assay to investigate the mRNA levels of the three novel POU-family members. Distinct and specific expression patterns for all three members were obtained in the different investigated organs. Interestingly the expression of the Pit- 1 related cDNA clone 5.80 was elevated in all developmental stages of the lactogenic-hormone dependent mouse mammary gland and in sceletal muscle, whereas its expression was low in other organs.

\section{S20-18}

\section{Stimulation of protein kinase $\mathbf{C}$ induces a mitogenic response in differentiated oligodendrocytes}

Pouly S. ${ }^{1}$, Monnet-Tschudi, F. 1 , Matthieu J.-M. ${ }^{2}$ and Honegger P.' 1 Institut de Physiologie, Université de Lausanne, 1005 Lausanne ${ }^{2}$ Laboratoire de Neurochimie, Pédiatrie, CHUV, 1011 Lausanne

Aggregating brain cell cultures prepared from 16-day rat embryos attain a high degree of cellular organization and differentiation. Within 4 weeks in vitro, the two oligodendroglial parameters, 2',3'cyclic nucleotide 3 '-phosphohydrolase (CNP) and myelin basic protein (MBP) attain maximal values, in good agreement with morphological findings indicating the presence of myelinated axons. After a single treatment of these cultures at day 26 with mezerein $(50 \mathrm{nM})$, a protein $C$ kinase activator, thymidine incorporation increased significantly, whereas total MBP content and CNP activity decreased drastically. These responses were found to be transient, since 20 days after stimulation higher MBP and CNP values were found in stimulated cultures, when compared to their untreated controls. These results suggest that protein kinase $\mathrm{C}$ activation induces de-differentiation and proliferation of myelinating oligodendrocytes, after which the responding cells are able to undergo extensive maturation. 
INFLUEANCE OF PRENATPAL DIAZEPAM ADMINISTTRATION ON REGIONAL DEVETLPMENT OF $K$-OPIOID RECEPTORS IN THE RAT BRATN AND PRODYNORPHIN IRRNA LEVEHS INN THE RAT NUCLEUS ACCUMBENSS.

Inderbitzin S., Schlumpf M., Lichtensteiger W., Institute of Pharmacology, University of zürich, CH-8057 Zürich

The influence of prenatal diazepam exposure $(1,25$ $\mathrm{mg} / \mathrm{kg} / \mathrm{d}, \mathrm{s.c.}$ ) from gestational day 14 to 20 on the development of the $\kappa$-opioid binding sites in striatum, nucleus accumbens and midbrain of PN 14, PN 28 and 8 week old male and female rats was studied. 8 week old prenatally diazepam exposed male rats showed a significant decrease of $\kappa$-opioid binding sites in the nucleus accumbens. A sex-dependent difference in $\kappa$-opioid binding site densities could also be detected between PN 28 prenatally diazepam exposed male and female rats. We now investigate by in situ hybridisation whether changes in the level of mRNA encoding for prodynorphin, the precursor for various endogenous $\mathrm{k}$-opioid agonists, could be responsible for the decrease of $\kappa$-opioid binding sites in the nucleus accumbens of 8 week old prenatally diazepam exposed male rats.

\section{S21-02}

\section{HIGH SENSITIVITY OF GLIAL CELLS TO A NEUROTOXIC} INSULT

Zurich, M.-G., Monnet-Tschudi, F. and Honegger, P.

Institut de Physiologie, Université de Lausanne, 1005 Lausanne.

Trimethyltin (TMT), a well-known neurotoxicant, was used to study the sensitivity of the different brain cell types (i.e. neurons, astrocytes, oligodendrocytes and microglial cells) to a neurotoxic insult. Aggregating brain cell cultures of fetal rat telencephalon were treated during 10 days with TMT at different concentrations, ranging from $10^{-10} \mathrm{M}$ to $10^{-6} \mathrm{M}$. Microglia were found to be the most sensitive cell type, since already at $10^{-10} \mathrm{M}$ of TMT an increased number and clustering of Griffonia simplicifolia-positive cells could be observed. At $10^{-8} \mathrm{M}$ of TMT astrocytes showed increased staining for glial fibrillary acidic protein, characteristic of gliosis. Neurons and oligodendrocytes appeared to be the least sensitive cells, since a decrease in the activity of cell type-specific enzymes was observed only at $10^{-6} \mathrm{M}$ of TMT. These results show that microglial cells and astrocytes respond to a toxic insult before any neuronal changes could be detected, and that the microglial reaction may be the first target of TMT. This reaction could then trigger the astrocytic response.

\section{$521-03$}

\section{CHARACTERIZATION OF VIP-INDUCED PROTEINS OF MOUSE ASTROCYTES BY 2-D GEL ELECTROPHORESIS.}

J.-R. Cardinaux and P.J. Magistretti. Institut de Physiologie, Université de Lausanne, Lausanne, Switzerland.

In primary cultures of mouse cerebral cortical astrocytes, a rapid glycogenolysis followed by a massive glycogen resynthesis ( six - to ten-fold over basal levels after $9 \mathrm{hr}$ ) are induced by vasoactive intestinal peptide (VIP) or noradrenaline (NA). Both actions of these neurotransmitters are mediated by cAMP. Since the induction of glycogen resynthesis triggered by VIP or NA is abolished by inhibition of transcription and translation, we applied the 2-D gel electrophoresis technique to search for astrocytic proteins induced by VIP or NA. The comparison of 35S-labeled proteins from primary astrocyte cultures treated or not with VIP $1 \mu \mathrm{M}$ revealed two newly synthesized proteins: a $36 \mathrm{kDa}$ protein $(\mathrm{pI} \approx 5)$ and a $23 / 24 \mathrm{kDa}$ protein $(\mathrm{pI} \approx 6)$. Only the latter is visible on a silver stained 2-D gel, which is a prerequisite to consider its purification and microsequencing. Induction of the $23 / 24 \mathrm{kDa}$ protein by VIP was time-dependent with a maximum at $\sim 12 \mathrm{hr}$. Preliminary results indicate a similar induction by NA and isoproterenol.
DISTRIBUTION AND MORPHOLOGY OF NITRIC OXIDE-POSITIVE NEURONS IN THE MARMOSET CEREBRAL CORTEX DURING PRE- AND POSTNATAL DEVELOPMENT

J.P. Hornung* and J. Schultz"* *Institute of Anatomy, University of Lausanne, 1005 Lausanne, and **University of Fribourg, 1700 Fribourg

Nitric oxide, synthesized by the enzyme nitric oxide synthase (NOS), is a neuroactive substance and a limited number of neurons were shown to express this enzyme either by histochemistry or by immunocytochemistry. Both techniques were performed on brains of marmosets with ages ranging from 6 weeks before birth to adult. The morphology and distribution of NOS-positive neurons were analyzed in all lobes of the cerebral cortex. The same pattern was revealed by histochemistry or immunocytochemistry. Three populations of NOS-positive neurons were revealed. First, a transient population of neurons in the molecular layer of the embryonic cortex, many having the morphology of the Retzius-Cajal cells. A second population was made of large multipolar neurons in the deep layers of the cortex and the underlying white matter, which persisted from embryonic to adult life. A third, permanent, population was made of small, weakly reactive neurons in the supragranular layers appearing postnatally. This study demonstrates that NO can exert its actions in the cerebral cortex through several pathways at different developmental stages.

\section{$\mathrm{S} 21-05$}

ONTOGENY OF VASOACTIVE INTESTINAL PEPTIDE (VIP) IN RAT FOREBRAIN.

M. Graber, J.-M. Burgunder, Neuromorphologisches Labor, Neurologische Klinik, universität Bern.

VIP is widely distributed in brain with suggested functions related to development. VIP expression was studied during rat brain development using hybridization histochemistry with a $48 \mathrm{mer}$ probe. VIP mRNA was found in thalamic nuclei on E17, later recognized as the ventrolateral and reticular nuclei after further maturation during prenatal period. VIP mRNA was found in hypothalamus, especially suprachiasmatic nucleus on $\mathrm{E} 19$ and its expression matured over the next days. Few cortical neurons contained VIP mRNA on E21, they continuously increased in number and signal intensity over the perinatal period. VIP gradually matured in the first three postnatal weeks and adult-like patterns were found on $\mathrm{P} 22$, when cerebral cortex, ventrolateral and reticular thalamic nuclei, hypothalamus, esp. suprachiasmatic nucleus, were the regions with most prominent VIP expression. These results demonstrate the relatively late appearance of VIP gene expression in the rat forebrain, as compared with peptides like SRIF and CCK, not suggesting a major role in early brain maturation.

\section{S21-06}

YOHIMBINE ANTAGONISM AS A TOOL TO ASSESS THE EXTENT OF MONOAMINERGIC MODULATION IN ANALGESICS EFFECTS - EXPERIENCE WITH TRAMADOL

Desmeules, J., Piguet, V., Collart, L., and Dayer, P. Division of Clinical Pharmacology \& Pain Clinic, University Hospital, CH-1211 Geneva 14. In humans, the central analgesic effect of tramadol $(T)$ is only weakly reversed by the $\mu$ opioid antagonist naloxone (Nx) (Br J Clin Pharmacol $1993 ; 35: 73$ P). T analgesia may thus result from an action on monoaminergic pathways as suggested by in vitro and animal data. We therefore investigated the effect of $\alpha_{2}$-adrenoceptor antagonism by yohimbine $(\mathrm{Y})$ on $\mathrm{T}$ analgesia. According to a randomised double-blind crossover and placebo $(P)$ controlled design, healthy volunteers $(n=10)$ received $\mathrm{T}(100 \mathrm{mg}$ orally), followed $(+3 \mathrm{~h})$ by $\mathrm{Y}(0.1 \mathrm{mg} / \mathrm{kg}$ intravenously), and $\mathrm{Y}+\mathrm{Nx}(0.8 \mathrm{mg}$ intravenously). Analgesia was assessed over 8 h by subjective pain threshold (numerical scale -NS-) and objective pain threshold (RIII nociceptive reflex -RIII-) monitoring. Peak analgesic effect was observed at ca. $3.25 \mathrm{~h}$ (RIII $+8.8 \pm$ SEM1.6 mA and NS $+8.4 \pm 1.8$ ) vs $P(R I I I-0.3 \pm 1.2$ and NS $+2.3 \pm 1.6, P<0.05)$ and lasted ca. $6 \mathrm{~h}$. Y reversed $\mathrm{T}$ analgesic effects during ca. $1 \mathrm{~h}$ (RIII $-2.9 \pm 1.8$, $\mathrm{P}<0.05$ and $\mathrm{NS}+2.8 \pm 1.5, \mathrm{P}<0.05$ ), whereas $\mathrm{Y}+\mathrm{Nx}$ abolished $\mathrm{T}$ effects thoughout the study period (RIII $-1.9 \pm 1.1$ and NS $+0.6 \pm 1.3, \mathrm{P}<0.05$ ). $\mathrm{Y}$ alone tended to lower pain thresholds (RIII $-4.1 \pm 1.5$ and NS $-1.9 \pm 1.4$, $\mathrm{P}>0.05$ ANOVA). Yohimbine, an $\alpha_{2}$-adrenoceptor antagonist, reverses tramadol effects, thus pointing to the major role of monoaminergic modulation in tramadol antinociception. 
S21-07

LOCALIZATION OF NEURITE GROWTH INHIBITORY PROTEINS IN THE CNS B.P. Rubin, J.P. Kapfhammer, I. Dusart* and M.E. Schwab, Institut für Hirnforschung der Universität Zürich, August Forel-Str. 1, CH-8029 Zürich, ${ }^{*}$ INSERM, U-106, Histol. Normale et Pathologique du Systeme Nerveux, Hôpital de la Salpétrière, 47, Boulevard de l'Hôpital, F-75651 Paris, Cedex 13 A monoclonal antibody (IN-1) was raised against neurite growth inhibitory proteins present in rat CNS myelin (Caroni and Schwab, Neuron 1: 85, 1988). IN-1 neutralizes the inhibitory effect of CNS tissue in vitro and in vivo, thus permitting regeneration of certain lesioned CNS fiber systems. We have used this antibody to immunostain cryostat sections of the nervous system of the rat. We found that IN-1 stains white matter and myelinated fiber tracts in the CNS. The staining pattern corresponds to that of the known myelin specific antigens MBP (myelin basic protein), MAG (myelin associated glycoprotein) and MOG (myelin oligodendrocyte glycoprotein). The staining of IN-1 in the CNS is found in regions of the adult nervous system which have been shown to be inhibitory for axonal growth. Interestingly, the regions which show a high abundance of IN-1 antigens are low in staining for GAP-43, a marker for growth and plasticity in the developing and adult brain. In contrast, GAP.43 is strongly expressed in unmyelinated fiber tracts or nuclei. Prevention of oligodendrocyte development in rat spinal cord resulted in suppression of $\mathrm{IN}-1$ expression and elevated GAP-43 levels. This suggests that inhibitory myelin proteins may negatively influence plasticity in the rat CNS.

\section{\$21-08}

ISOLATION, PRIMARY STRUCTURE AND METAL BINDING PROPERTIES OF NEURONAL GROWTH INHIBITORY FACTOR (GIF) FROM BOVINE AND EQUINE BRAIN

Faller, P., Fundel, S., Pountney, D.L. and Vašák, M., Biochemisches Institut der Universität Zürich

Human neuronal growth inhibitory factor (GIF) is involved in the regulation of neuronal growth and is deficient in the brains of Alzheimer's disease victims. This brain-specific metalloprotein consists of 68 amino acids out of which 20 are Cys and has been found to contain copper and zinc. Proteins with similar primary structure were isolated from bovine and equine brain. The amino acid sequence of these proteins shows about $70 \%$ homology to those of mammalian metallothioneins (MT), with two conserved inserts of $1 \mathrm{Thr}$ and a glutamic acid rich hexapeptide in the $\mathrm{N}$ - and $\mathrm{C}$-terminal regions, respectively. In contrast to MTs, which usually contain only $\mathrm{Zn}(\mathrm{II})$, the presence of $\mathrm{Cu}(\mathrm{I})$ and $\mathrm{Zn}(\mathrm{II})$ (6-7 moles total) in all GIF isolations suggests the functional importance of both metals ions. To spectroscopically probe the native $\mathrm{Zn}$-binding sites, the $\mathrm{Zn}(\mathrm{II})$ ions were replaced by $\mathrm{Cd}(\mathrm{II})$. Both bovine and equine $\mathrm{Cd} / \mathrm{Cu}-\mathrm{GIF}$ derivatives exhibit similar absorption and $\mathrm{CD}$ spectra with features characteristic of Cd-thiolate clusters.

\section{S21-09}

\section{Multicell culture system for the study of drug kinetics}

Wechsler D., *Schitny J.C. and Honegger U. E., Depts. of Pharmacology and *Anatomy, University of Bern, CH-3010 Bern

A cell culture system was developed for the investigation of cellular drug kinetics in cultures with up to 4 cell types. It was used for the study of uptake, competitive uptake, release and redistribution of drugs. Individual cell types (human skin fibroblasts, rat astrocytoma cells (C6) and rat hybridoma cells (oligodendrocyte $x$ astrocytoma, ROC)) were separately grown to confluency on glass circle sectors. In the same petri dish 4 sectors in various combinations were exposed to media containing radiolabelled chlorpromazine (CPZ). Single and repetitive uptake and release of CPZ were measured in each cell type after individual exposure or exposure in any combination of cell types. In 2 hour competitive uptake studies fibroblasts reached 1.7 and 2.6 times the concentrations of $\mathrm{C} 6$ - and ROC-cells, respectively. In redistribution experiments the exchange of CPZ from preloaded to unloaded cells was tested. The exchange rate was dependent on cell type and loading period. It decreased with increasing time of exposure suggesting the formation of more stable CPZ stores.
$S 21-10$

Immunohistochemical localization of ANP and NPY in the vertebrate adrenal organ

M. Wolfensberger and M. Reinecke, Institute of Anatomy, University of Zürich, Winterthurerstr. 190, CH-8057 Zürich

To date, the existence of ANP and NPY in the adrenal medulla has been shown only for a few mammalian and anuran species. Thus, the present immunohistochemical investigation attempts to localize ANP-like and NPY-like peptides in the adrenal gland of representative mammals, birds, reptiles, amphibia and bony fish by the use of antisera specific for mammalian ANP and NPY. Furthermore, the catecholamine-containing cells were characterized by antisera against enzymes of catecholamine synthesis. ANP- and NPY-immunoreactivies were detected in adrenal chromaffin cells of all vertebrates studied while no immunoreactivities were observed in the adrenal cortex or in its homolog, the interrenal. The representatives of the different vertebrate classes exhibited marked differences in the proportions of ANP-immunoreactive and NPY-immunoreactive cells in the presence of ANP- and NPY-immunoreactivities in noradrenalineand/or adrenaline-containing cells and in the amount of of cells containing both ANP- and NPY-immunoreactivities. Our results give evidence for a long phylogenetic history of adrenal ANP- and NPY-like peptides. Thus, they stress the physiological impact of these peptides as adrenal paracrine and/or endocrine hormones.

S21-11

GLUTAMATE-EVOKED RELEASE OF ARACHIDONIC ACID FROM MOUSE CORTICAL NEURONS IN PRIMARY CULTURES N. Stella \& P. J. Magistretti, Institut de Physiologie, Université de Lausanne.

The release of ${ }^{3} \mathrm{H}$-arachidonic acid evoked by glutamate was characterized in cerebral cortical neurons in primary culture grown under conditions that prevent glial cell proliferation. In these cultures, $99 \%$ of the total cells are immunocytochemically characterized as being positive for specific neuronal markers such as neuron-specific enolase and neurofilament. Specific markers for astrocytes, oligodendrocytes or microglia were not detected. Glutamate induces a concentration-dependent release of ${ }^{3} \mathrm{H}$-arachidonic acid with a EC50 of $3 \mu \mathrm{M}$. The pharmacological profile of this glutamate response shows the involvement of two receptor types: the NMDA and the AMPA ionotropic receptors. The glutamate-evoked release of ${ }^{3} \mathrm{H}$-arachidonic acid is $\mathrm{pH}$ sensitive. When the incubation buffer is alkalinized from 7.25 to 7.65 , both basal and glutamate evoked release of ${ }^{3} \mathrm{H}$-arachidonic acid are enhanced. The glutamate response is also enhanced as intracellular $\mathrm{pH}$ is alkalinized by pharmacological treatments: such as inhibition of $\mathrm{Cl}^{-} / \mathrm{HCO}^{-}$antiport by DIDS. These results further stress the role of intracellular $\mathrm{pH}$ in glutamate-mediated neurotransmission.

\section{$\$ 21-12$}

LACTATE IS THE MAJOR METABOLIC SUBSTRATE RELEASED BY ASTROCYTES. O. Sorg and P.J. Magistretti. Institut de Physiologie, Université de Lausanne.

We have previously demonstrated that certain neurotransmitters such as VIP, noradrenaline (NA) and adenosine promote glycogenolysis in mouse cerebral cortical astrocyte cultures (Brain Res. 563: 227-233, 1991). The question that we then addressed was the nature of the metabolic substrate released by astrocytes following glycogenolysis. We therefore determined the presence of various metabolic substrated released from astrocytes under static conditions and in the superfusate of a laminar flow system developed in our laboratory. Neither glucose nor any intermediate of the tricarboxylic acid cycle could account for the decrease in glycogen stores; astrocytes however were shown to release great quantities of pyruvate and lactate at a rate of $50 \mathrm{nmol} / \mathrm{mg}$ protein/minute. Noradrenaline but not VIP promotes a significant increase $(42 \%)$ in lactate release. The effect of noradrenaline is mimicked by isoproterenol and inhibited by propranolol, suggesting a $\beta$ adrenergic mediation. When astrocytes are incubated in an isotonic solution containing no energy substrate, the glycogen stores are rapidly depleted and lactate, but not glucose, is released in the medium. In conclusion, lactate appears to play a major role in cerebral energy metabolism homeostasis, as substrate released by astrocytes to provide energy fuel for neurones and other neighboring cells. 
$521-13$

COMPARATIVE LIGHT AND ELECTRON MICROSCOPY STUDY OF CALRETININ-EXPRESSING CELLS IN THE PINEAL GLAND OF HUMAN AND MAMMALS

Novier A., Nicolas D., Krstic R., Institut d'Histologie \& d'Embryologie, Bugnon 9, 1005 Lausanne

It is known so far that calcium-binding proteins (CaBPs: calbindin, parvalbumin and calretinin) are expressed in some cells of the pineal gland of laboratory mammals. The exact phenotype of these cells is unknown. Nevertheless, according to recent studies, it seems that some of them are ganglion cells. Therefore wo studied the pineal gland of different species (gerbils, rats, goats, cows and humans) using calretinin (CR) antibody which is considered as marker for neurons (Andressen $\&$ al, Cell \& Tissue Res, 271:181, 1993).

The CR-expressing cells were found in all our species: in gerbils, rats, cows and humans they were scattered throughout the parenchyma, whereas in goats they lined mostly the pericapillary spaces. According to our findings, it seems that most of the reactive cells are rather neuron-like elements since morphological criteria for true neurons (axosomatic synapses, Nissl bodies, bundles of neurofilaments) are missing. However one cannot exclude that among these cells some are intrapineal ganglion cells, because of Ranvier's nodes found along the processes of some CRpositive cells.

Thus, it is possible that these cells are involved in the modulatory control mechanism of the pineal neurohumoral activity and/or by accumulation of intracellular calcium in the formation of pineal calcifications.

S21-14

MODULATION OF SYNAPTIC TRANSMISSION BY VIP IN MOUSE CINGULATE CORTEX IN VITRO

Pralong, E., Kiraly, M. and Magistretti, P.J. Institut de Physiologie, Université de Lausanne, CH-1005 Lausanne.

Vasoactive intestinal peptide (VIP) induces long lasting metabolic effects in the mouse CNS. We have investigated a shorter neuromodulatory action of VIP on the synaptic responses in coronal slices of adult mouse cingulate cortex by means of intracellular current clamp recordings. Electrical stimulation of the underlying white matter evoked a multiphasic synaptic potential cousisting of early EPSP, early IPSP, late EPSP and late IPSP mediated by non-NMDA, NMDA, $\mathrm{GABA}_{\mathrm{A}}$ and $\mathrm{GABA} \mathrm{B}_{\mathrm{B}}$ receptors, respectively. VIP $(0.1 \mu \mathrm{M})$ superfused for $5 \mathrm{~min}$. had no effect, whereas concentrations over $0.2 \mu \mathrm{M}$ selectively and reversibly depressed the NMDA-mediated responses in 12/16 cells. In the presence of CNQX $(10 \mu \mathrm{M})$ and bicuculline $(10 \mu \mathrm{M})$, the isolated NMDA-mediated late EPSP was still attenuated by $0.3 \mu \mathrm{M}$ VIP in $8 / 10$ cells (mean:- $42 \%$ after $30 \mathrm{~min}$.). In 3 other cells VTP induced a spreading depression (SD) and a strong, reversible inhibition of the late EPSP (mean:-64\% after $10 \mathrm{~mm}$.). These results indicate that in the absence of GABAergic inhibition VIP can induce two types of effects in the mouse cingulate cortex, a prolonged decrease of the NMDAmediated synaptic response and a striking SD.

\section{S21-15}

\section{PROTEIN KINASE A AND C POTENTIATE INHIBITORY SYNAPTIC TRANSMISSION IN THE HIPPOCAMPUS M. Capogna, B. H. Gähwiler and S. M. Thompson}

Brain Research Institute, University of Zurich, CH-8029 Zurich Presynaptic proteins involved in neurotransmitter release (i.e. synapsin, $\mathrm{B}-50$ ) and some postsynaptic $\mathrm{GABA}_{\mathrm{A}}$ receptor subunits possess phosphorylation sites for CAMP-dependent protein kinase (PKA) and/or protein kinase $\mathrm{C}$ (PKC). The functional consequences of phosphorylation by these kinases is not known. We have studied the action of specific activators of PKC and PKA, phorbol ester (PDBu) and forskolin, respectively, on GABAergic synaptic transmission in area $\mathrm{CA} 3$ of hippocampal slice cultures. PDBu significantly enhanced the amplitude of evoked monosynaptic IPSCs (in CNQX and AP5), and both PDBu and forskolin increased the frequency of spontaneous miniature IPSCs (mIPSCs) in the presence of CNQX, APS and TTX. This effect by $\mathrm{PDBu}$ was antagonized by staurosporin, a protein kinase inhibitor. PDBu and forskolin did not change the amplitude or decay of mIPSCs, suggesting that only presynaptic kinases are involved. Furthermore, PDBu enhanced mIPSC frequency by a comparable amount in the presence of the $\mathrm{Ca}^{2+}$ channel blocker $\mathrm{Cd}^{2+}$, indicating that PDBu did not enhance GABA release from presynaptic endings by facilitating $\mathrm{Ca}^{2+}$ influx into axon terminals.
S21-16

RELEASE OF HOMOCYSTEIC ACID FROM PRIMARY ASTROCYTE CULTURES: ROLE AS GLIOTRANSMTTTER. ${ }^{1}$ K.Q.Do, ${ }^{2}$ O.Sorg, and ${ }^{2} \mathbf{P}$. Magistretti. ${ }^{1}$ Brain Res.Inst, Uni. Zürich; ${ }^{2}$ Inst.Physiology, Uni. Lausanne.

Besides the exeitatory amino acid transmitter glutamate, its sulfur containing analogue homocysteic acid (HCA) could play a role in excitatory processes in the central nervous system. HCA is present in and released from nervous tissue and is a potent neuronal excitant, predominantly activating $\mathrm{N}$ Methyl-D-Aspartate receptors (Do et al., 1992). These properties are suggestive of a classic synaptic neurotransmitter role. On the other hand, HCA seems to be localized not in neurones but in glial cells (Grandes et al., 1991; Tschopp et al. 1992). The efflux of HCA is temporally delayed following activation of specific pathways (Klancnik et al., 1992); these latter observations are not in accordance with the classic concepts of synaptically-mediated neurotransmission. A laminar flow superfusion system of monolayer cultures was developed and the released materials from mouse cortical astrocytes, previously preincubated with [ ${ }^{35}$ S]-methionine, were investigated. During application of either noradrenaline or the $\mathrm{B}$-adrenergic agonist isoproterenol, the extracellular level of $\left[{ }^{35} \mathrm{~S}\right]$-methionine was decreased and that of labelled HCA was increased. This effect was not observed with the -adrenergic agonist methoxamine, and could be blocked by the B-adrenergic antagonist propanolol. These results, combined with the delayed HCA release, the glial localisation of HCA and its neuroactivity, strongly suggest a potential role of HCA as a gliotransmitter, modulated by noradrenaline inputs.

$\mathrm{S} 21-17$

Interaction between VIP and glutamate on $c$-fos mRNA expression. I.-L. Martin, D. Gasser and P.J. Magistretti. Institut de Physiologie, Université de Lausanne, Lausanne, Switzerland. The regulation of $c$-fos mRNA expression by VIP and glutamate was examined in primary cultures of neurons originating from the mouse cerebral cortex. In primary cultures of cortical neurons, VIP increases cAMP levels and stimulates $c$-fos mRNA expression. Interestingly VIP stimulation is completely blocked by MK-801, an NMDA receptor antagonist but not by CNQX or AP3, which antagonize AMPA/kainate and metabotropic receptors respectively. $c$-fos expression stimulated by glutamate shares the same pharmacology. These results suggest an involvement of endogenously released glutamate in the stimulation of $c$-fos mRNA expression evoked by VIP. Furthermore, when added together, VIP and glutamate interact synergistically to increase $c$ fos mRNA levels. Consistent with the pharmacology of VIP and glutamate, the synergistic interaction between VIP and glutamate on $c$-fos mRNA expression is also inhibited by MK-801. Interestingly, this synergism is completely inhibited by $\mathrm{H}-89$, a protein kinase $A$ inhibitor, whereas staurosporin and $\mathrm{KN}-62$ which block protein kinases $\mathrm{C}$ and $\mathrm{Ca}^{++} /$calmodulin dependent respectively exhibit smaller inhibitory effects.

\section{S21-18}

BLOCYTRS OF ARACHIDONIC ACID CATABOIISM IMPAIR TAF CURRIAT ACROSS CARDTAC GAP JUNCTIONS

Valiunas, V., Schmilinsky Fluri, $G$. and Weingart, $R$. Dept. of Physiology, University of Bern, $C H-3012$ Bern Arachidonic acid (AA) reversibly impairs the gap junction conductance $\left(g_{f}\right)$ in cardiac cells. Now, we examined whether AA acts direct Iy or via its catabolites. Experiments were performed on pairs of neonatal rat myocytes using a dual voltage-clamp method. We used blockers which interfere with enzymes of various catabolic pathways. Pretreatment with 10 HM POCA (blocks carnitine acyltransferase I) did not prevent the decline in $g$, by $10 \mu \mathrm{M} \mathrm{AA}$; i.e. intermediates of $\beta$-oxidation are not involved. Similarly, preexposure to $10 \mu \mathrm{M}$ indomethacin (blocks the cyclooxygenase pathway) did not prevent $A A$ from uncoupling; this rules out an involvement of prostaglandins and thromboxanes. Exposure to $10 \mu \mathrm{M}$ NDGA (blocks the 5-lipoxygenase pathway) per se produced a decrease in $g_{j}$. Likewise, exposure to $10 \mu \mathrm{M}$ ETYA (blocks the 15-1ipoxygenase pathway) also impaired $g$. These effects cannot result from accumulation of endogenous $A A$ because preexposure to $50 \mu \mathrm{M}$ $4 B P B$ (blocks phospholipase $A_{2}$ ) did not prevent NDGA- or ETYA-induced uncoupling. Exposure to $75 \mu \mathrm{M}$ SKF 525-A (blocks the epoxygenase pathway) also produced a decrease in $g_{f}$. Hence, NDGA, ETYA and SKF 525-A, compounds with different biochemical actions and of different chemical structure, act on $g_{3}$ either directly or via an unknown mechanism. 
$521-19$

POTENTIAL CHANGES ASSOCIATED WITH ELECTRICAL ACTIVITY IN NONMYELINATED NERVE FIBRES

A. Robert and P. Jirounek

Département de Pharmacologie, CMU, 1211 Genève 4

Simulteneous measurements of the extracellular concentration of $\mathrm{K}^{+}$ $\left(\left[\mathrm{K}^{+}\right]_{\mathrm{e}}\right)$, and of the concomitant changes in the membrane potential $\left(E_{m}\right)$ were measured in the rabbit vagus nerve during and after a period of activity. During a $15 \mathrm{~Hz}$ stimulation there was a large increase in the $\left[\mathrm{K}^{+}\right]_{\mathrm{e}}$, accompagned by a change in $\mathrm{E}_{\mathrm{m}}$, which roughly followed the depolarization expected from the increase in $\left[\mathrm{K}^{+}\right]_{\mathrm{e}^{-}}$At the end of the stimulation, although the $\left[\mathrm{K}^{+}\right]_{\mathrm{e}}$ was still elevated, the nerve developed a positetanic hyperpolarization (PTH). The PTH was generated by the electrogenicity of the $\mathrm{Na}^{+}-\mathrm{K}^{+}$pump, but its amplitude and time-course were strongly affected by two depolarizing currents: $(i)$ an inward $\mathrm{Ba}^{2+}$-sensitive current of $\mathrm{K}^{+}$and (ii) an outward current of $\mathrm{Cl}^{-}$. We hypothesize that during activity and during the early phase of recovery there was a $\mathrm{Ba}^{2+}$-sensitive uptake of potassium by the Schwann cells. The $\mathrm{Cl}^{-}$current, by short-circuiting the $\mathrm{Na}^{+}-\mathrm{K}^{+}$pump, contributes to the control of the $\mathrm{E}_{\mathrm{m}}$, and by this way to the driving force for the inward $\mathrm{K}^{+}$current.
S21-20

ANALYBIS OF 2-CHLOROETHYLAMINE (CEA) IN URTNE OP IFOSFAKIDE (IFO) TREATED PATIENTS

C. Aeschlimarn, T. Oerry, A. Klpfer. Dept. of Clinical Fharmacology, University of Berne and Dept. of Medical onoology, Inselspital, Berne, Switzerland.

The metabolite pattern of the antineoplatic drug ifosfanide has been studied in the past. Fomal release of CFA has been postulated on theoretical grounds but positive identification of this compound in patient urine has not been actieved so far. Recently, an aocidental overdose of ifosfamide has coared in a cancer patient (25g i.v. IFO/24 hours with $20 \mathrm{~g}$ mesna as ungarotectant). Urine collection was obtained in 24 hour intervals for two days. CEA was measured by a gas liquid ctranatography method of the USP XXII (Suppl. V, pag.26272629) using a phosphoraus and nitrogen sensitive themionic detector. In this patient the urinary elimination of CEA was $1500 \mathrm{mg} / 24 \mathrm{~h}$ and $207 \mathrm{mg} / 24 \mathrm{~h}$ at day one and day two respectively. Subsequently CFA has also been found in patients under high dose ifosfamide treatment $\left(16 \mathrm{~g} / \mathrm{m}^{2} / \mathrm{cycle}\right.$ ). When CFA was adninistered to rats $(100 \mathrm{mg} / \mathrm{kg}$ or $200 \mathrm{mg} / \mathrm{kg}$ intraperitoneally), no CFA could be detected in urine within 24 hours after drug actministration. From these preliminary data we conclude that CEA is extensively metabolized in rats and the mechanism of CEA elimination in patient urine might reflect a functional derangement of CFA metabolism in man secondary to ifosfamide co-administration.

\section{Behaviour}

\section{S22-01}

\section{ENHANCED SPATIAL LEARNING ABILITIES FOLLOWING PRE AND POSTNATAL CHOLINE TREATMENT}

C. BRANDNER, F. SCHENK, Institut de Physiologie, $\mathrm{CH}-1005$ Lausanne.

There are numerous reports of improved memory functions in rats following a dietary supplementation with choline. In some cases, this improvement can be related to enhanced cholinergic transmission (Meck et al., Behav Neurosci., 103, 1234-1241, 1989). But it is not clear whether there is a general improvement in memory or whether specific aspects of learning and memory are affected. This work was aimed at analysing the effects of perinatal cholinergic supplementation on the development of spatial abilities and upon adult performance.

Choline supplementation $(3.5 \mathrm{~g} . /$. in $0.02 \mathrm{M}$ saccharine solution in tap water) was maintained during two weeks before birth. Additional supplementation was maintained from the 5 th to the 10 th week postnatal. Spatial learning capacities were studied at the ages of 26, 65 or 80 days in a circular swimming pool (Morris place navigation task) and at the age of of 7 months on a homing board. Adult and aged rats were tested on a radial maze.

Selective aspects of the performance were markedly improved. This treatment had no specific effect upon spatial memory per se, but, instead, affected learning abilitles by promoting efficient behavioural strategies hypothetically based on active representation and anticipation processes. The effects of the treatment remained evident up to 6 months following the supplementation.

\section{S22-02}

\section{FIXED RATE VS SUBJECT PACED RAPID INFORMATION PROCESSING}

\section{Hasenfratz, B. Baldinger, K. Bättig}

Behavioral Biology Laboratory. Swiss Federal Institute of Technology, CH 8092 Zürich, Switzerland

Two groups of abstinent female smokers, performing either a fixed rate or a subject paced version of a rapid information processing task were compared. Both groups participated in two test sessions (in which the task was performed twice) to compare two different types of cigarettes which were smoked before and during the second task trial: a) the subject's habitual cigarette with a nicotine yield of at least $0.7 \mathrm{mg} /$ cigarette and b) a nearly nicotine free test cigarette with a tar yield comparable to that of the habitua cigarette. Reaction times to correct detections (series of three odd or even digits in a pseudorandom sequence of single digits) were longer with the subject paced version and not affected by the type of cigarette but shorter with the fixed rate version and even more decreased with the smoking of the habitual cigarette. On the other hand, the pre- to posttreatment increase in the subject paced processing rate was greater with the habitual than the test cigarette. It was concluded that the two task versions assess different cognitive functions. Whereas the fixed rate version primarily assesses vigilance performance and seems to be more suitable to measure changes in reaction time, the subject paced version is more sensitive to changes in speed capabilities in rapid information processing.
S22-03

BEHAVIORAL EFFECTS OF PRENATAL (PN) DIAZEPAM IN ROMAN LOW- AND HIGH-AVOIDANCE (L,H) RATS.

Driscoll, P, Fernández-Terue T,A, Escorihuela,RM and

Tobeña,A. ETHz, Nutztierwissenschaften, CH-8092

Zurich \& Autòn. Univ. Barcelona, E-08193 Bellaterra

Females of both 1 ines ( $n=8 / \mathrm{grp}$ ) were injected $\mathrm{sc}$ daily between days 15-20 of pregnancy with vehicle (V), 1(D1) or 3(D3) $\mathrm{mg} / \mathrm{kg}$ diazepam, or not(C). In these studies $(A, B)$ their $5-7$ mo old offspring were subjected to a $5 \overline{0}$-run session in a shuttlebox ( $A: 12$ males, 6 females of each 7 ine/condition) or a $\overline{6}$ min session in a hexagonal tunnel maze with strategically-placed barriers and a 1 it central arena(B:6-7 males of each line/condition). A: The freezing behavior of LD3 rats was reduced VS LC, this behavior reverting more to escapes in males \& avoidances in females, although $V$ had an effect in the latter also. No within-line differences in inter-trial responses were seen, but pre-session activity was increased in female LVs and LD1s, returning to LC levels for LD3s. No differences existed among the $H$ groups. B: Whereas $H$ maze activity exceeded that of $L$, no within-line differences appeared. HD3s entered the lit arena less than HCS or HVS, indicating an increased timidity after PND, and a trend in the same direction existed for LDI/LD3 vs LV.

\section{S22-04}

\section{NICOTINE-DEPENDENT AND NON-NICOTINIC EFFECTS OF SMOKING}

\section{B. Baldinger, M. Hasenfratz, K. Bättig}

Behavioral Biology Laboratory, Swiss Federal Institute of Technology, $\mathrm{CH}-8092$ Zürich, Switzerland

Heart rate, physical activity, and cigarette consumption were continuously recorded under field conditions, whereas subjective craving was assessed six times per day and saliva cotinine was measured once daily. Abstinence, habitual cigarettes (with a nicotine yield of at least $0.7 \mathrm{mg} / \mathrm{cigarette)}$, and nearly nicotine free test cigarettes but with a tar yield comparable to that of the habitual cigarettes were compared in a sample of twelve female smokers for two days each. Whereas physical activity was similar for the three conditions, heart rate was highest with the habitual clgarettes, lowest on abstinence days and in between with the test cigarettes. Cigarette consumption was similar for both types of cigarettes and subjective craving was higher on abstinence than on smoking days but no differentiation between the two cigarette types was obtained. Saliva cotinine values were highest on the days with the habitual cigarettes but lower and similar with the test cigarettes and on abstinence days. It was concluded that heart rate and saliva cotinine depended only on the amount of nicotine absorbed, whereas subjective craving was reduced by smoking independently of the actual nicotine yield of the cigarette. 


\section{Biochemistry and Physiology}

S23-01

6-PYRUVOYL TETRAHYDROPTERIN SYNTHASE: CHARACTERIZATION, CRYSTALLIZATION AND X-RAY STRUCTURE OF A CLINICALLY RELEVANT ENZYME. D. Bürgisser, H. Nar ${ }^{*}$ and $C$. W. Heizmann, Division of Clinical Chemistry, Department of Pediatrics, University of Zürich, $\mathrm{CH}-8032$ Zürich,"Max Planck Institut für Biochemie, D-8033 Martinsried/München

6-Pyruvoyl tetrahydropterin synthase (PTPS) is the rate limiting enzyme in the synthesis of $\mathrm{BH}_{4}$ in man, a cofactor for several hydroxylases involved in catecholamine and serotonin biosynthesis. The human and rat liver cDNAs encoding the $16 \mathrm{kDa}$ subunit of PTPS were expressed and the recombinant enzymes purified to homogeneity. The apparent $\mathrm{K}_{\mathrm{H}}$ for the substrate, pI and heat stability of the re-combinant enzymes were similar to the native enzymes. The rat enzyme was crystallized and the $x$-ray structure showed a hexameric native form arranged as a sandwich of two trimers. Three histidine, a glutamic acid, and - also shown by site-directed mutagenesis - a cysteine residue characterize the active center of the enzyme thought to be $\mathrm{Mg}^{2+}$-dependent. According to the proposed ligands we replaced $\mathrm{Mg}^{2+}$ by $\mathrm{Fe}^{2+}$ or $\mathrm{Mn}^{2+}$ and observed an enhanced activity up to $100 \%$.

\section{S23-02}

\section{EXPRESSION, PURIFICATION AND CHARACTERIZATION OF THE RECOMBINANT KRINGLE (2+3) DOMAINS OF HUMAN PLASMINOGEN}

\author{
S.Söhndel, M. Affolter, J. Schaller and E.E. Rickli \\ Institute of Biochemistry, University of Bern
}

Kringle 2 and kringle 3 of human plasminogen are the only kringle domains connected by an interkringle disulfide bond. They are so far not available by limited proteolysis.

The kringle $(2+3)$ domains $(K 2+3)$ were successfully expressed in E. coli. A N-terminal hexahistidine tag was fused to insure the isolation of $\mathrm{K} 2+3$ by affinity chromatography on a $\mathrm{Ni}-2+-\mathrm{NTA}-$ agarose-column. To be able to remove the hexahistidine tag a factor Xa cleavage site was introduced between the 6 histidine residues and the $\mathrm{N}$-terminus of the kringle structures.

The correct arrangement of the disulfid bonds was determined by amino acid- and sequence analysis.

The lysine binding site was proven by affinity chromatography on lysine-Bio-Gel, as previously shown for K2. The dissociation constant of $\mathrm{K} 2+3$ was measured by intrinsic fluorescence titration with 6-aminohexanoic acid.

\section{S23-03}

UPTAKE AND RELEASE OF HEXACHLOROBIPHENYL AND CHLOROPROMAZINE IN 3T 3 -PREADIPOCYTES AND 3T $_{3}$-ADIPOCYTES IN VITRO

S. Mühlebach and U.E. Honegger, Dept. of Pharmacology, University of Bern, CH-3010 Bern (Switzerland)

$2,4,5,2^{\prime}, 4^{\prime}, 5^{\prime}$-hexachlorobiphenyl (6-CB) shows limited elimination and extensive storage in adipose tissue in vivo (lipophilic unmetabolizable compound) while chlorpromazine (CPZ) is known for its lysosomal storage. Uptake and release of 6-CB and CPZ were studied in 3T 3 -preadipocytes $(\mathrm{P})$ and $3 \mathrm{~T}_{3}$-adipocytes $(\mathrm{A})$. $3 \mathrm{~T}_{3}$-Cells were cultivated in Dulbecco's minimal essential medium supplement with $10 \%$ fetal calf serum and grown to confluency. Differentiation was stimulated by dexamethason and bezafibrate exposure for $48 \mathrm{~h}$. Radiolabelled 6-CB $(20 \mu \mathrm{M})$ or $\mathrm{CPZ}$ $(5 \mu \mathrm{M})$ was added to the medium. Following a single dose $70-80 \%$ of $\mathrm{CPZ}$ were taken up within an hour into $P$ and $A$. In contrast, 6-CB reached an intracellular plateau of $30-50 \%$ of the dose after $1 \mathrm{~h}$ in $\mathrm{P}$ whereas dependent on the triglyceride content $\mathrm{A}$ accumulated up to $95 \%$ of the dose 6-CB uptake into A was temperature dependent and not saturable with repetitive daily doses up to 1 week. 6-CB uptake into $P$ was fully reversible while the release from $A$ was limited to $10 \%$ of the accumulated drug. In contrast, the release of $C P Z$ was higher from $P$ than from $A$ as a consequence of the different lysosomal activities of the respective cells. The use of $3 \mathrm{~T}_{3}$ cells allowed to show a remarkable difference in cellular handling of neurral and basic lipophilic drugs and may be helpful to further elucidate basic mechanisms involved.
S23-04

Differential expression of the conversion endoproteases PC1(3) and PC2 in pancreatic islet cell types

M. Neerman-Arbez, V. Cirulli and P. Halban. Laboratoires Jeantet. Centre Médical Universitaire, 1211 Genève 4.

PC1(3) and PC2, members of the mammalian family of pro-protein convertases homologous to the yeast kex 2 , are both expressed in pancreatic islets of Langerhans and are thought to be responsible for the conversion of proinsulin to insulin and C-peptide in beta cells. However, the insulin secreting beta cells are not the only cells present in these complex microorgans, prompting us to evaluate the expression of $\mathrm{PC} 1$ and $\mathrm{PC} 2$ in islet beta and non-beta cells. Rat islet cells were sorted by autofluorescence activated flow cytometry to separate beta cells from non-beta cells and conversion endoprotease levels were analysed by Western blotting. In beta cells, PC1 levels were higher than $\mathrm{PC} 2(\mathrm{PC} 1 / \mathrm{PC} 2=2.6 \pm 0.2)$ whereas the opposite was found for non-beta cells ( $\mathrm{PC1} / \mathrm{PC} 2=0.05 \pm 0.04$ ), confirming that $\mathrm{PC1}$ is important for proinsulin conversion while suggesting a role for $\mathrm{PC} 2$ in the conversion of proglucagon, prosomatostatin and propancreatic polypeptide. Transformed murine cell lines (insulin-producing beta-TC and glucagonproducing alpha-TC) were found to faithfully reflect the primary rat cells in terms of their $\mathrm{PC} 1 / \mathrm{PC} 2$ ratios. Finally, post-translational modification of the convertases themselves was found to differ between cell-types, in particular, a precursor $75 \mathrm{kD}$ form of $\mathrm{PC} 2$ accumulated in beta cells whereas only the fully processed $67 \mathrm{kD}$ form was detected in the non-beta cells.

\section{S23-05}

\section{A replication protein A copurifying DNA helicase from calf thymus \\ Anthl Georgakl, Narendra Tuteja, Birgit Sturzenegger and UIrich Hobscher \\ Department of Veterinary Biochemistry University of Zürich-Irchel Winterthurerstrasse $190 \mathrm{CH}-8057$ Zürich, Switzerland}

A DNA helicase from calf thymus copurified with replication protein $A$ through several steps of purification including DEAE-Sephacel, hydroxyapatite and single stranded DNA cellulose. It is finally separated from replication protein $A$ on FPIC Mono Q where the DNA helicase elutes after replication protein A. We named this new calf thymus enzyme DNA helicase $F$. Characterization of the helicase $F$ by affinity labeling with $\left[\alpha^{32}\right.$ P]ATP indicated that the enzyme has a catalytic subunit of $72 \mathrm{kDa}$. Gel filtration experiments suggested that DNA helicase $F$ can exist both in a monomeric and an oligomeric form. The enzyme unwinds in the $5^{\prime}-3^{\prime}$ direction in relation to the DNA strand it binds. All eight deoxyribonucleoside- and ribonucleosidetriphosphates could serve as an energy source. Testing a variety of DNADNA substrates indicated that the DNA helicase $F$ preferentially unwinds very short substrates and is slightly stimulated by a single stranded 3'-tail. Replication protein A allowed the DNA helicase $F$ to unwind longer DNA substrates up to 400 bases suggesting that copurification of replication protein $A$ with the DNA helicase might be of functional relevance.

S23-06

Chronic granulomatous disease: An attempt to reconstitute the function of the $\mathrm{X}$-linked form of the disease.

Shila D. Schneider*+, Johann.-P. Hossle*, Walter Schaffnert, Sandro Rusconit, * University Children's Hospital, Division of ImmunologyIHematology, Zurich;+ Institute for Mol. Biol.II of the University of Zurich

Chronic Granulomatous Disease (CGD) is a group of congenital immunodeficiencies that predispose patients to recurrent severe bacterial and fungal infections. The cause of the disease is lack or impairment of O2- production in phagocytic cells due to mutations in any of the four components of the superoxide producing system. Approximately $70 \%$ of all CGD patients suffer from an X-linked form of the disease where the phagocyte oxidase gp91phox is affected. This project concentrates on the reconstitution of the deficient gene in phagocytic patient cells. Expression of recombinant $g p 91$ was assayed in vitro and in vivo. Attempts to "tag" $g p 91$ with a foreign epitope were unfortunately so far unsuccessful. The target cells for gene reconstitution are non-dividing monocytes. Thus, we have chosen an Adenovirus over other viral systems because of: a) its ability to infect nondividing cells, b) its genetic stability and, c) lack of human adenovirus related malignancies or side-effects. We constructed two recombinant Adenoviruses: one recombinant expresses a $\mathrm{LacZ}$ gene (control), another the gp91phox gene. We are trying the expression of recombinants AdenolLacZ and Adeno/gp 91 in normal and patient-derived cells (for example EBVtransformed normal and patient B-cell lines), as well as the reconstitution of NADPH oxidase function in peripheral blood monocytes of CGD patients. 
KINETICS OF MOLECULAR CHAPERONE ACTION

Schmid, D., Gehring, H., "Baici, A. and Christen, D. Biochemisches Institut der Universität Zürich, CH8057 Zürich; "Rheumaklinik, Universitässpital, CH8091 Zürich

Molecular chaperones of the Hsp70 type transiently sequester unfolded segments of proteins and promote their correct folding. Target peptides labelled with an environmentally sensitive fluorophore allowed to follow their binding to the molecular chaperone Dnak of Escherichia coli in real-time. The two-step process is characterized by relaxation times $\tau_{1}=27 \mathrm{~s}$ and $\tau_{2}=200 \mathrm{~s}$ at $2 \mu \mathrm{M}$ DnaK and 0.1 $\mu \mathrm{M}$ ligand at $25^{\circ} \mathrm{C}$. In the presence of ATP, the formation of the complex is greatly accelerated and follows a single-exponential process with $\tau=0.4$ s. The rate of dissociation of the complex was even more increased than that of association resulting in a decreased net affinity for ligands in the presence of ATP. The binding-release cycle of Dnak thus occurs in the time range of polypeptide chain elongation and folding and is too fast to be stoichiometrically coupled to the ATPase activity of the chaperone $\left(\mathrm{k}_{\mathrm{cot}}=0.13 \mathrm{~min}^{-1}\right.$ at $\left.30^{\circ} \mathrm{C}\right)$. Supported by the olga Mayenfisch Stiftung, Zurich, and the Hartmann Mülier-Stiftung, zurich.

\section{$\$ 23-08$}

DOMAIN MOVEMENT IN ASPARTATE AMINOTRANSEERASE SIMULATED BY MOLECULAR DYNAMICS Kasper, P., Sterk, M., Christen, P. and Gehring, H., zürich

Two 120-ps molecular dynamics simulations were performed on aspartate aminotransferase (2x401 amino acid residues). The simulations started from the crystal structures of the holo enzyme (open form) and the liganded enzyme (closed form), both placed in a 5-A shell of water. The consistent valence force field of the program DISCOVER was used. The rms values of the individual Ca deviations agreed well with the corresponding B-factors of the crystal structures. superposition analysis of the trajectories of both simulations and of the corresponding initiai structures indicated a hinge bending motion between the large and the small domain of each subunit. The simulation with the open form showed a displacement of the small domain towards the conformation of the closed crystal structure. Significantly smaller movements were observed in the simulation with the liganded enzyme. The structural differences between the protein in the crystal and the ensuing calculated structures might arise from the absence of lattice forces in solution.

\section{\$23-09}

EVOLUTIONARY RELATIONSHIPS AMONG PYRIDOXAL-5'-PDEPENDENT AMINO ACID DECARBOXYIAASES

Sandmeier, E., Hale, T.I. and Christen, P.

Biochemisches Institut der Universität Zürich, 8057 Zürich.

The pyridoxal-5'-P (PLP)-dependent amino acid decarboxylases (DC) can be subdivided into four apparently unrelated groups. Group $I$ is represented by glycine DC, group II comprises glutamate, histidine, tyrosine and aromatic-L-amino acid DC, group III procaryotic ornithine and lysire DC as well as the procaryotic biodegradative type of arginine DC, group IV eucaryotic ornithine and arginine $D C$ as well as the procaryotic biosynthetic type of arginine DC and diaminopimelate DC. $(N-1)$ Profile analysis, a more stringent application of profile analysis [Gribskov et al. (1990) Methods Enzymol. 183, 146-159] established the homology among the enzymes of each group. A search with the profile of group II indicated a distant relationship with aminotransferases and thus with the $\alpha$ family of PLP-dependent enzymes. No evidence was obtained that groups I, III and IV are related with other PLP-dependent enzymes or any other protein in the database. Apparently, the amino acid DC are of multiple evolutionary origin, in some cases even if they have the same substrate specificity.
Comparison of the activities of native bovine seminal ribonuclease and that secreted from $\mathrm{E}$. coli Schein, $\mathrm{CH}^{*}$ (SIAT, Technopark, Pfingstweidstr. 30, CH8005 Zürich) and Haugg, M (Lab. Org. Chemistry, E.T.H., CH-8092).

Seminal ribonuclease (BS-RNase) differs from the pancreatic RNase $A$ in that it forms a cysteine-linked dimer and has a relatively higher specific activity in the cleavage of double-stranded (ds-) RNA substrates. We expressed BS-RN in a secretion system similar to that described previously (Biochem. J. 283:377-344, 1992) using the signal sequence of murine pancreatic ribonuclease. About $1 \mathrm{mg}$ BS-RN was isolated per liter culture supernatant.

Human and bovine recombinant inferferon- $\gamma$ (IFN- $y$ ) inhibit the degradation of ss- and ds-RNA by bovine pancreatic RNase while they activate the activity of BS-RN on the same substrates (FEBS Letters, 270:229-332, 1990). Recombinant bovine or murine pancreatic RNase (produced in our secretion system) are inhibited by IFN- $y$, while the recombinant BS-RN or a cysteine-linked dimer of RNase $A$ is also activated by IFN- - .

IFN-y activates BS-RN even in the presence of inhibitory concentrations (0.1-1 mM) of mononucleotides. Thus the mode of activation cannot be attributed to alleviation of product inhibition. Kinetic studies using 300 BP ds-RNA as substrate suggest that IFN- $y$ interacts directly with the BS-RN to increase the rate of highmolecular weight product/substrate release, as the apparent $\mathrm{K}_{\mathbf{S}}$ increases proportionately to the increase in $\mathrm{k}_{\text {cat }}$.

S23-11

CYCIOSPORTN A POTENTIATES VASOPRESSIN-INDUCEO RTSE IN $\left[\mathrm{Ca}^{2+}\right]_{i}$

A. Lo Russo, A.C. Passaquin, U.T. Rülegg,

Ecole de Pharmacie, Univ. Lausanne, CH-1015 lausanne

Drug-induced local vasoconstriction appears to be responsible for the hypertensive side effect of the immunosuporessant cyclosporin A (CSA). We have therefore examined the $\left[\mathrm{Ca}^{2+}\right]_{i}$ increase caused by the vasoconstrictor hormone $\left[\mathrm{Ang}^{\mathrm{B}}\right]$ vasopressin (AVP) in vascular smooth muscle cells (VSMC) treated with CSA.

$\left[\mathrm{Ca}^{2+}\right]_{i}$ levels were monitored either with a fluorescence analyser using fura 2 or by ${ }^{45} \mathrm{Ca}^{2+}$ efflux.

Pretreatment of VSMC with CSA increased the AVP incuced rise in $\left[\mathrm{Ca}^{2+}\right]_{i}$. A leftward and upward shift of the concentration-response curve of the AVP-induced rise in ${ }^{45} \mathrm{Ca}^{2+}$ efflux was also doserved after CSA treatment. The induction of CSA potentiation was detectable after $3 \mathrm{~min}$, took 1 to $2 \mathrm{~h}$ to become fully established and was reversed after washout. CSA potentiation was concentration-dependent with a threshold at $10^{-7} \mathrm{M}$.

This potentiating effect of CSA may be the underlying cause for CsA-induced hypertension.

Supported by the Swiss National Science Foundation (grant Nr.31-36514.92) and Sandoz Phanna.

S23-12

CLONING OF THE ACTIN GENE OF NEUROSPORA CRASSA

Wuethrich $G .{ }^{1}$, Maeder B. ${ }^{2}$, Bolivar I. ${ }^{3}$, Barja, F. I and Turian, G. I, ILab. Gen. Microbiology, 2 Dept. Biochemistry, 3 station Exp. Zoology, University of Geneva, 30 Quai Ernest-Ansermet, 1211 Geneva 4

The actin has been found from the simplest forms of cell to the highly evolved ones. In higher organisms it is transcribed by multigene families but in yeast and several filamentous fungi there appears to be only one actin gene. It was therefore of interest to study the expression of actin gene in $N$. crassa in which three actin isoforms have been characterized (Barja et al., 1991, FEMS Lett, 77:1924). After isolating genomic DNA from wild type $N$. crassa a PCR was performed with primers corresponding to actin consensus sequences and an amplified fragment of the gene (verified by sequencing) was obtained. This fragment was used as a probe in a Southern to assess the number of actin gene(s). Our results suggest that $N$. crassa has also only one actin gene. 
THE EPITOPE RECOGNIZED BY THE $\alpha$-SMOOTH MUSCLE ACTIN ANTIBODY IS IMPORTANT FOR POL YMERIZATION IN VITRO AND IN VIVO. Chaponnier C., M. Goethals, F. Gabbiani, G. Gabbiani and J. Vandekerckhove. Department of Pathology, University of Geneva, Switzerland and Laboratory of Physiological Chemistry, State University of Ghent, Belgium.

The blocking effect of the $\mathrm{N}$-terminal decapeptide of $\alpha$-smooth muscle actin (SMA) (Ac.EEEDSTALVC) on the monocional antibody anti- $\alpha$ SM-1 was compared with that of synthetic peptides modified by changing the $\mathrm{N}$-terminal acetyl group or by substituting a single amino acid in position 1 to 5 . Using immunofluorescence or immunoblotting techniques, anti- $\alpha S M-1$ activity was abolished after incubation with the native peptide and peptides modified in position 1 (only when $E \rightarrow A$ ) or 5 . On immunoblots running BSA crosslinked peptides on denatured gels, only the natural peptide and peptides with substitution in position 1 or 5 were delected by the antibody. Our results indicate that AC. (E)EED is the epitope for anti- $\alpha S M-1$. Binding of anti- $\alpha S M-1$ to SMA increased actin polymerization by decreasing the critical concentration. As control this action was not exerted on skeletal actin. This is the first example of the role of a precise $\mathrm{N}$-terminal sequence in the polymerization of a single actin isoform. Double immunofluorescence for $\alpha$-SMA and total actin on cultured aortic SM cells microinjected with the native peptide showed a selective disappearance of $\alpha$-SMA staining suggesting that this peptide traps a protein involved in $\alpha$-SMA polymerization. Work is in progress to search for the putative actin-binding protein(s) physiologically interacting with the $\mathrm{N}$-terminal sequence of $\alpha-S M A$. (Supported by the Swiss National Science Foundation Grant \# 31-30796.91 and 31-34062.92 and a grant from the Concerted Actions (OOA) of the Flemish Community)

\section{S23-14}

\section{HYDROLYSIS OF HIGH MOLEKULAR WEIGH'T KININOGEN BY PLASMA KALIIKREIN}

Benner, S. and Dutler, H., Laboratory for Organic Chemistry, ETH Hönggerberg, CH-8093 Zürich

High Molecular Weight Kininogen (HMWK) is cleaved by the serineprotease plasma kallikrein to generate the hypotensive peptide bradykinin in human blood. We established a measuring system for the time course of the hydrolysis of HMWK in its native and deglycosylated form and in the presence or absence of CI-inhibitor. Analysis of the bradykinin release and the yielded protein fragments lead to the three following results:

1. The hydrolysis did not follow plane Michaelis-Menten kinetics due to a HMWK-fragment operating as a competitive inhibitor. 2. Addition of the $\mathrm{C} \overline{1}$-inhibitor stopped the HMWK-hydrolysis immediately, which is in contrast to the physiological role of plasma kallikrein in blood.

3. So far we have no evidence that the high content of O-glycosylated side-chains of the HMWK-light chain has an effect on the kinin-liberation as proposed in literature.

(SNF N 20-30836.91/2)

\section{S23-15}

EFFECT OF PHOSPHOLIPASE A2 ON THE RATE AND EXTENT OF PHOSPHATIDYLGLYCEROL MOLECULAR SPECIES HYDROLYSIS IN SPINACH AND SQUASH THYLAKOIDS

Xu, Y.N., Meylan, M. and Siegenthaler, P.A., Laboratoire de Physiologie végétale de l'Université, CH-2000 Neuchãtel

The thylakoid membrane (TM) contains 10 molecular species of phosphatidylglycerol (PG). The relative amount of these species depends on the plant species and growth conditions. Using phospholipase $\mathrm{A} 2\left(\mathrm{PLA}_{2}\right)$ at $0^{\circ} \mathrm{C}$ to digest preferentially $P G$ in the outer monolayer of spinach $T M$, we have shown previousiy that this monolayer was enriched in PG (ca 70\%). The purpose of this investigation was to determine the transmembrane distribution of each PG molecular species. To this aim, we have studied the hydrolysis kinetics of each species in the presence of PLA in spinach and squash TM containing different relative proportion of molecular species. The hydrolysis rate and extent of the molecular species depended on the unsaturation degree of the fatty acid at the sn-1 position as well as on the presence or absence of $16: 1(3 t)$ at the sn-2 position. For instance, the hydrolysis rate of $P G$ $18: 3 / 16: 1(3 t)$ was greater than that of PG $16: 0 / 16: 0$. These results $w i l l$ be discussed in terms of the thylakoid transmembrane distribution of each PG molecular species. (Supported by the SNSP 3100-33693.92).
Mitochondrial Lon Protease is Required for Maintaining Respiratory Function in Yeast. Carolyn K. Suzuki, Kitaru Suda, Nan Wang*, Michael M. Gottesman* and Gottfried Schatz. Biozentrum der Universität Basel, CH-4056 Basel, *National Institutes of Health, Bethesda, MD 20892 USA

Within mitochondria, the mechanism of selective protein degradation is poorly understood. While the bulk of mitochondrial proteins are long-lived, some are degraded rapidly. The selective degradation of mitochondrial proteins is likely to be mediated by proteases similar to those found in bacteria; this is based on the hypothesis that mitochondria have evolved from bacterial endosymbionts, in conjunction with the finding that mammalian mitochondria contain an ATP-dependent proteolytic activity similar to that in bacteria. One ATP-dependent protease from bacteria, Lon, is of particular interest because it is involved in regulated protein turnover.

Degenerate oligonucleotide primers corresponding to two regions of the bacterial Lon gene were used to PCR amplify a $1 \mathrm{~kb}$ fragment from yeast DNA that encoded an open reading frame with striking similarity to the bacterial Lon protease. By screening a yeast cDNA library, we isolated a 3.6 $\mathrm{kb}$ cione potentially encoding a protein of 1133 amino acids. Haploids bearing a distuptedLON gene were unable to respire or to grow on ethanol/glycerol. Fractionation of mitochondrial matrix from wild-type cells revealed a peak of ATP-dependent proteolytic activity which was absent in the disruptant. Furthermore, we have identified matrix proteins that are degraded with a half-time of $\sim 60 \mathrm{~min}$ in intact wild-type cells but are stable in the lon disruptants. Electron microscopy of lon disruptants revealed the presence of many electron dense bodies in the mitochondrial matrix which are not found in Lon $^{+}$cells.

S23-17

MOLECULAR CHARACTERIZATION OF A RECESSIVE AUTOSOMAL DISORDER CAUSING TETRAHYYROBIOPTERIN DEFICIENCY

Thöny, B., Leimbacher, W., Blau, N. and Heizmann, C.W. Division of Clinical Chemistry, Department of Pediatrics, University of Zürich, Steinwiesstrasse 75, 8032 Zürich, Switzerland

Tetrahydrobiopterin $\left(\mathrm{BH}_{4}\right)$ is the essential cofactor for the hepatic phenylalanine hydroxylase, but also for tyrosine and tryptophan hydroxylases that are responsible for the production of neurotransmitter precursors. Furthermore, $\mathrm{BH}_{4}$ is required for the cleavage of glyceryl ethers and is involved in the biosynthesis of nitric oxide by the nitric oxide synthase. A vatiant type of hyperphenylalaninemia is caused by a deficiency of $\mathrm{BH}_{4}$. The most frequent form of this cofactor deficiency is due to lack of 6-pyruvoyl-tetrahydropterin synthase (PTPS) activity. The human cDNA for PTPS was isolated, and the recombinant protein was found to be active when expressed in $E$. coli, thus allowing us to perform functional testing of mutant proteins. Primary skin fibroblasts derived from several PTPS deficient patients were investigated for the molecular nature of this autosomal recessive disorder. All fibroblasts had PTPS mRNA expressed, and subsequent cDNA sequence analysis revealed different mutations in the coding region of PTPS (codon replacements, deletions, or nonsense codons) responsible for a lowered or no enzyme activity. In order to potentially correct PTPS activity (and restore $\mathrm{BH}_{4}$ biosynthesis) we are establishing a recombinant retroviral-mediated gene transfer system to efficiently introduce the wild-type human PTPS-cDNA in these fibroblasts.

S23-18

EPITHELIAL CELL TOXICITY DUE TO METAL IONS: ROLE OF $\left[\mathrm{Ca}^{2+}\right]_{i}$.

P. Hausel, J. Diezi. Inst. de Pharmacologie et de Toxicologie, Université de Lausanne.

From previous electrophysiological evidence, we concluded that metal ions $\left(\mathrm{Hg}^{2+}, \mathrm{Cd}^{2+}, \mathrm{Ag}^{+}\right)$at low concentrations $\left(10^{-6} \mathrm{M}\right)$ increase rapidly $(10-60 \mathrm{~s})$ cation $\left(\mathrm{Na}^{+}, \mathrm{K}^{+}\right.$) conductance at the apical membrane of epithelial cells. We investigated here the effects of these metals on $\left[\mathrm{Ca}^{2+}\right]_{i}$ in MDCK-I cultured cells, for a potential correlation between functional membrane changes and $\left[\mathrm{Ca}^{2+}\right]_{i}$. Metals $\left(10^{-4}-10^{-6} \mathrm{M}\right)$ were added at the apical side of the epithelium, and $\left[\mathrm{Ca}^{2+}\right]_{i}$ was measured with fura-2.

$\mathrm{Hg}^{2+}$ induced a rapid (peak 5-10 s) and marked increase in $\left[\mathrm{Ca}^{2+}\right]_{i}$, whereas $\mathrm{Cd}^{2+}$ entailed a slower (peak 2-5 min) and marked response. The time-course of effects for both metals was similar to the electrophysiological changes. In contrast, the pattern of effects of $\mathrm{Ag}^{+}$on $\left[\mathrm{Ca}^{2+}\right]_{i}$ differed markedly from that on apical membrane conductance. Thus, the effects of metals on $\left[\mathrm{Ca}^{2+}\right]_{i}$ are conspicuous but not tightly associated with changes in membrane conductance. 
PURIFICATION DF RAT BRAIN PLASMA MEMBRANE DEHYDROGENASES

C. BULLIARD and J.-L. DREYER, Institut de Biochimie, Université de Fribourg, CH-1700 Fribourg

Trans-plasma membrane oxydoreductases (PMO) play a significant role in energy transduction and post-receptor signal transmission, but the enzymes have not been characterized so far. We have achieved the purification of PMO from rat brain synaptic vesicles and from synaptic plasma membranes. The membrane enzymes were extracted by $1 \%$ Lubrol XI TM, precipitated with ammonium sulfate (between 40 and $70 \%$ saturation) and purified by means of hydrophobic chromatography on butyl-agarose and gel filtration on Superose-12, followed by PAGE under native conditions. Specific enzymatic staining of native PAGE gels yields two homogenous diaphoraselike activities, PMO-I and PMO-II . SDS-PAGE of the enzymes showed that PMO-I is made of two subunits of $52 \mathrm{kDa}$ and $40 \mathrm{kDa}$ whereas PMO-II consists of a single sub-unit of $52 \mathrm{kDa}$. The $52 \mathrm{kDa}$ subunits from PMO-I and PMO-II are probably identical from their respective properties. Partial $\mathrm{N}$-terminal sequencing (13 AA) of the $40 \mathrm{kDa}$ subunit from PMO-I showed $84 \%$ homology with rat brain fructose-1,6-bisphophate aldolase. These data indicate that PMO is closely associated with the glycolytic enzyme that co-purifies in all steps under suitable conditions. The biochemical functions of PMO's remain to be established.

\section{S23-20}

\section{EUIDENCE FOR AN NADH-DIAPHORASE LOCATED AT THE CELl PLASMg MEMBRANE IN NB41A3, A MOUSE NEUROBLASTOMA CELL LINE}

R. ZURBRIGGEN and J.-L. DREYER, Institut de Biochimie, Université de Fribourg, $\mathrm{CH}-1700$ Fribourg

Most mammalian cells display transplasma membrane oxidoreductase activity (PMO). The enzymes use intracellular reduced pyridine nucleotide to reduce an unspecific extracellular electron acceptor as substrate. In order to set up a methodology for purifying, characterizing and quantifying PMO's, the plasma membrane from a neuroblastoma cell line, NB41A3 has been biotinylated. Subsequently the biotinylated proteins were purified by immunoprecipitation with avidin, antiavidin-antibodies and insoluble protein $A$. The protein recovery of an immunopurified membrane preparation was $<0.15 \%$ of the protein content in the cell extract. Specific PMO activity was increased 15 to 20 fold compared to the activity in whole cells. This approach demonstrates the presence of PMO within the cell plasma membrane. This activity accounts for about one third of the total cellular diaphorase activity and cannot be attributed to an increased permeabilizarion of the plasma membrane induced upon biotinylation nor activity from lysed cells. low substrate concentrations $(0.1-1 \mu \mathrm{M})$. Native gel electrophoresis of iminobiotinylated and affinity purified plasma membrane extracts displays two diaphorase-positive bands, indicating that a homogeneous cell population may express several PMO activities at the plasma membrane.

$\$ 23-21$

\section{PREDICTING IN VIVO DRUG INTERACTIONS FROM IN VITRO} DATA: FLUVASTATIN AND P450TB (CYP2C9) SUBSTRATES

Transon, C., Leemann, T., and Dayer, P. Division de Pharmacologie clinique, Hôpital Cantonal Universitaire, $\mathrm{CH}-1211$ Genève 14

Fluvastatin (FS) is a new HMG-CoA reductase inhibitor. Its affinity for 3 major human drug metabolizing P450 monooxygenases (CYP2C9, CYP2D6 and CYP3A4) was determined in liver microsomes. FS showed low affinity $(\mathrm{Ki}>50 \mu \mathrm{M})$ for CYP2D6 and CYP3A4 whereas CYP2C9 was selectively and competitively inhibited $(\mathrm{Ki} \approx 0.1 \mu \mathrm{M})$. The potential for in vivo FS interactions with CYP2C9 substrates was therefore investigated in 14 volunteers using diclofenac (DF) as a probe $(25 \mathrm{mg}$ orally) on days 0,1 and 8 . DF and 4'-HO-DF kinetics were determined by HPLC/UV. DF $C_{\max }$ increased over time (0.28 [SD 0.12], 0.38 [0.20] and $0.45[0.4] \mathrm{mg} / \mathrm{L}$ on day 0,1 and 8 , resp.). Oral clearance was reduced on days 1 and $8(14 \%$ and $15 \%$, resp.). A time dependent decrease in urinary metabolic ratio (4'-HO-DF/DF) was noted (1.07 [0.34], $0.90[0.23]$ and $0.70[0.18]$ on day 0,1 and 8 , resp. $(p<0.0001))$ only over the first 4 hours. Fluvastatin therefore exhibits a two-phase interaction in vivo: an early transient and a late cumulative inhibition. The transient phase matches inhibition profiles predicted by quantitative models integrating in vivo pharmacokinetics and in vitro inhibition data (Q-DIPS). Simple competitive inhibition by FS cannot explain the late phase. Other mechanisms (i.e. FS or metabolite cumulation, MI complexation) are hypothesized. In some situations FS is expected to cause interactions with CYP2C9 substrates, which are partially predicted by quantitative modeling of in vitro data (SNSF Project No 32-36600.92).
ISOLATION AND CHARACTERIZATION OF CDNA CLONES CODING FOR THE FERREDOXIN:THIOREDOXIN REDUCTASE Gaymard, E., Marc-Martin, S., Spielmann, A., Stutz, E. and Schürmann, P., Laboratoire de Biochimie végétale, Université, $\mathrm{CH}-2000$ Neuchâtel

Ferredoxin:thioredoxin reductase (FTR) is the key enzyme in the ferredoxin/thioredoxin system, the light-dependent regulatory system in oxygenic photosynthesis. It is a nucleus encoded ironsulfur protein, composed of two dissimilar subunits, one of which is the catalytic subunit containing a redox-active disulfide bridge functional in the reduction of thioredoxins and a $4 \mathrm{Fe}-4 \mathrm{~S}$ cluster. Using PCR we have isolated and characterized a CDNA clone of $738 \mathrm{bp}$ from spinach and a clone of $911 \mathrm{bp}$ from corn coding for the catalytic subunits including the transit peptides. The first 31 (spinach) resp. 38 (corn) amino acids after the start exhibit typical features of chloroplast transit peptides. Following the transit peptides are 113 (spinach) or 114 (corn) amino acids representing the catalytic subunits. The primary structures are highly conserved between these two species with $91 \%$ similarity and $81 \%$ homology. Seven of the eight Cys residues found in the spinach subunit and important for the catalytic activity are at conserved positions. (SNF 31-28811.90 and 31-37725.93)

\section{S23-23}

\section{CATION BINDING TO RAT PARVALBUMIN MUTANTS WITH DEFECTIVE CALCIUM-BINDING SITES.}

T.L. Pauls ${ }^{i}$, I. Durussel' ${ }^{2}$, M.W. Berchtold', J.A. Cox $^{2}$.

'University of Zürich-Irchel, 8057 Zürich, Switzerland; ' ${ }^{2}$ Univerisité de Genève, 1211 Genève, Switzerland.

Previously, a parvalbumin mutant, $\mathrm{PV}_{\mathrm{F} 102 \mathrm{~W}}$, was constructed with a unique reporter Trp in the middle of the hydrophobic center. In the present study three new parvalbumin mutants, derived from $P V_{5102 w}$ and containing alterations essential for $\mathrm{Ca}^{2+}$-binding in either one, $\mathrm{PV}_{-\mathrm{CD}}$ and $\mathrm{PV} \mathrm{EEF}_{-\mathrm{F}}$, or both, $\mathrm{PV}_{-\mathrm{CD} / \mathrm{FF}}$, of the two $\mathrm{Ca}^{2+}$-binding sites, were analyzed in order to study the metal binding properties of individual $\mathrm{Ca}^{2+}$-binding domains and their contributions to the structure and stability of the protein. Both, $P V_{-C D}$ and $P V_{-E F}$, bind $1 \mathrm{Ca}^{2+}$ with affinity constants, $\mathrm{K}_{\mathrm{Ca}}$, of $1.1 \cdot 10^{7}$ and $3.2 \cdot 10^{6} \mathrm{M}^{-1}$ in the absence of $\mathrm{Mg}^{2+}$. $\mathrm{Mg}^{2+}$ shifts the $\mathrm{Ca}^{2+}$-binding isotherms of $\mathrm{PV}_{-\mathrm{CD}}$ to higher free $\left[\mathrm{Ca}^{2+}\right]$ according to the competition equation with $K_{M g}=2 \cdot 10^{3} \mathrm{M}^{-1}$. PV $\mathrm{PVF}_{-\mathrm{F}_{\mathrm{F}}}$ is much less effected by $\mathrm{Mg}^{2+}\left(\mathrm{K}_{\mathrm{Mg}}=80 \mathrm{M}^{-1}\right)$ and can be considered as possessing a $\mathrm{Ca}^{2+}$-specific site. Nevertheless, in the absence of $\mathrm{Ca}^{2+}$ both, $\mathrm{PV}_{-\mathrm{CD}}$ and $\mathrm{PV} \mathrm{EFF}_{\mathrm{EF}}$, bind 1 mole of $\mathrm{Mg}^{2+}$ with much higher affinity than predicted from the competition studies. $\mathrm{PV}_{-\mathrm{CD} / \mathrm{EF}}$ binds neither $\mathrm{Ca}^{2+}$ nor $\mathrm{Mg}^{2+}$. Trp-fluorimetry and UV-difference spectroscopy revealed that the $\mathrm{Ca}^{2+}$-loaded conformations of $\mathrm{PV}_{-\mathrm{CD}}, \mathrm{PV}_{-\mathrm{EF}}$ and $\mathrm{PV}_{\mathrm{F} 102 \mathrm{w}}$ are similar, with the Trp-102 deeply buried in the hydrophobic core. However, striking differences between the mutants are found in the metal-free and $\mathrm{Mg}^{2+}$-loaded forms. The conformation of $\mathrm{PV}$ or $\mathrm{Mg}^{2+}$. Our results indicate that destroying the $\mathrm{Ca}^{2+}$-binding of the EF loop, but not of the $\mathrm{CD}$ loop, strongly destabilizes the protein in the absence of $\mathrm{Ca}^{2+}$.

\section{$\$ 23-24$}

EXPRESSION OF A SYNTHETIC SEA URCHIN METALLOTHIONEIN GENE AND METAL BINDING PROPERTIES OF THE RECOMBINANT PROTEIN

Wang, Y., Mackay, E.A., Kurasaki, M., and Kägi, J.H.R. Biochemisches Institut der Universität Zürich, Switzerland, CH-8057.

The sea urchin, Strongylocentrotus purpuratus, metallothionein gene, MTa, was constructed and expressed under the control of a heat shock promoter in a protease-deficient strain of $E$. coli. The nascent recombinant protein was stabilised by the addition of exogenous $\mathrm{Cd}$. The Cd-containing protein was precipitated with ethanol and subsequently purified to homogeneity by gel permeation and ionexchange chromatography. The purified protein was identified as the desired gene product by tryptic peptide map analysis, sequence analysis and mass spectroscopy. Typical yields of protein were $2 \mathrm{mg}$ per litre of culture. The presence of $7 \mathrm{Cd}$ ions per molecule was confirmed by the SH/Cd ratio and by the existence of $7{ }^{113} \mathrm{Cd}$ NMR resonances. The apparent association constants of sea urchin MT

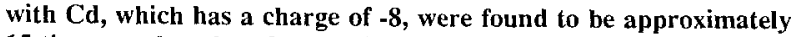
15 times weaker than for rabbit MT, at pH 7. 


\section{METALLOTHIONEINS : SEQUENCE ALIGNMENT AND EVOLUTION}

P.A. Binz, T.I. Hale, J.H.R. Kägi, Biochemisches Institut der Universität zürich

Metallothioneins (MT) are small metalloproteins displaying as their main feature clusters of $d^{10}$ metal ions bound to the thiolate ligands of the abundantly occurring cysteine residues (Cys). From a set of over 85 sequences a general multialignment of 62 class I MT has been obtained on the basis of the Needleman \& Wunsch algorithm. All Cys are conserved in the mammals and over $83 \%$ of them in other vertebrates and invertebrates. On the basis of similarity/identity scores we can isolate groups of sequences. Evolutionary relationships between species and groups have been calculated with the maximum parsimony method of Felsenstein and with the distance matrix method of Fitch and Margoliash. The divergences observed among the mammalian MTs indicate a partition into four distinct subforms which all may have arisen before the separation of the species in evolution and which in part may differ in function.

S23-26

CHARACTERISATION OF A 47 kDa HAEM- AND ZINCCONTAINING PROTEIN FROM BOVINE BRAIN

Fundel, S.M., Pountney, D.L. and Vašák, M., Biochemisches Institut der Universität Zürich, $\mathrm{CH}-8057$ Zürich

An acidic haem- and zinc-containing protein has been isolated from bovine brain. Native and SDS-polyacrylamide-gel-electrophoresis reveal a monomeric protein with an apparent molecular weight of $47,2 \mathrm{kDa}$. The absence of co-staining with tetramethylbenzidine implies that the haem group is non-covalently bound. The electronic absorption spectrum, with a Soret-band absorption maximum at 412 $\mathrm{nm}$ and $\alpha$ - and $\beta$-bands at 540 and $575 \mathrm{~nm}$, respectively, is similar to that of $\mathrm{Fe}(\mathrm{II})$-myoglobin, consistent with the presence of haemiron in the ferrous oxidation state. In air the protein was easily oxidized to the $\mathrm{Fe}$ (III) form with a subsequent shift of the Soretband maximum to $405 \mathrm{~nm}$. Atomic absorption measurements indicate approximately 1 mole of zinc and 1 mole of iron per mole of protein. The amino acid composition is similar to that of indoleamine-2,3-dioxygenase, although no such enzymic activity has been detected.

\section{\$23-27}

\section{OLIGONUCLEOTIDE IMMOBILIZATION BY LIGHT}

Küng, M. and Sigrist, $H$.

Institute of Biochemistry, University of Berne, CH-3012 Berne, Switzerland

The goal of covalent immobilization of oligonucleotide capture probes to solid supports has been accomplished by light-dependent, photolinker polymer mediated immobilization strategies. Synthetic oligonucleotides were immobilized by facile layer coating procedures. The procedure does not require functionalization of the oligonucleotide probe or the material surface. Oligonucleotides were linked to polystyrene with a biopolymer which was multiply substituted by photoactivatable diazirines. Capture probe photoimmobilization (350 $\mathrm{nm}$ irradiation) was strictly light and diazirine dependent. Using radiolabeled oligonucleotides as capture probes and poiystyrene as material surface, the light dependent coupling efficiency was $40 \%$. Nonspecific oligonucleotide binding was below $2 \%$ Photoimmobilized oligonucleotides retained the ability to form stable complexes with complementary DNA strands, and target oligonucleotides of varying length were captured as well as DNA fragments produced by PCR techniques.
HEXACHLOROBENZENE AT LOW DOSES PRODUCES LESIONS IN NONHUMAN PRIMATE OVARY

Bourque, A., Singh, A., Dykeman, A., MacMahon ${ }^{*}$, A., and Foster", W. Atlantic Veterinary College, Charlottetown, Canada, and *Reproductive Toxicology Section, Environmental Health Centre, Tunney's Pasture, Ottawa, Canada.

Hexachlorobenzene ( $\mathrm{HCB}$ ) is a known environmental pollutant that is produced as an industrial by-product of certain chemicals. When administered in high dosage, HCB induces alterations in the rhesus monkey ovary. A purpose of the study was to determine a no observable adverse effect level (NOAEL) for the compound. Twenty, cynomolgus monkeys, 6-13 years old were placed in five groups. $\mathrm{HCB}$ was given orally in concentrations of $0.01,0.1,1.0$, and $10 \mathrm{mg} / \mathrm{kg} \mathrm{b.w}$. in gelatin capsules, daily, for 13 weeks. One group of animals fed glucose only, served as the control. Ovary specimens fixed in $2 \%$ buffered glutaraldehyde were prepared by conventional methods for transmission electron microscopy. Ultrastructural alterations were revealed in the developing ova and follicular cells in all the ovaries from HCB-treated animals in a dose-related manner. Clinical chemistry was unaffected by HCB. A NOAEL for this reproductive toxicant is yet to be determined.

A.B. was a recipient of the NSERC Undergraduate Student award.

S23-29

OXO-XANTHURENIC ACID FORMATION IN BOVINE LENS

X. Martin \& H. Malina, Dept. of Ophthalmology, University of Zurich

3-Hydroxykynurenine (3-HK) acts as UV-B filter in the human lens. In this study, we looked for this substance and its metabolites in young and old bovine lenses, because of their possible role in cataract formation. The substances were detected by HPLC analysis. The kynurenine aminotransferase (KAT) activity was determinated by the method of Tobes (Methods Enzymol 1987; 142:217). The fluorescent substance formed from 3-HK was characterized by thin layer chromatography followed by reaction with ninhydrin, UV and fluorescence spectrometry, and atom bombardment for molecular mass determination. $3-\mathrm{HK}$ at concentrations of $0.08 \pm 0.01,0.2 \pm 0.04$, and $1 \pm 0.4 \mu \mathrm{g} / \mathrm{g}$ of tissue was detected in iris/ciliary body, retina, and calf lens respectively. This substance is deaminated by KAT. The activity of this enzyme was $2.6 \pm$ $0.3,3.7 \pm 0.2$, and $9.6 \pm 2 \mu \mathrm{mol} / \mathrm{g}$ of tissue/hour in retina, iris/ciliary body, and lens respectively of old bovine eyes, but it was not found in calf eyes. The deamination of $3-\mathrm{HK}$ resulted in the formation of a fluorescent substance which was identified as oxo-xanthurenic acid (OXA) with a molecular mass of 205 Daltons. The accumulation of OXA acid possibly interacting with lens proteins could induce cataract formation. SNF 32-36058.92, EMDO, and Hartmann-Müller.

$\$ 23-30$

IDO AND KAT ACTIVITIES IN HUMAN CATARACT H. Malina \& X. Martin, Dept. of Ophthalmology, University of Zurich

Indoleamine 2,3-dioxygenase (IDO), a superoxide radical scavenger, is present in transparent human lenses and produces 3-hydroxykynurenine, a UV-B filter. The synthesis of 3-hydroxykynurenine by IDO and its degradation by kynurenine aminotransferase (KAT) were measured in transparent lenses and cataracts. Post-mortem eyes of elderly persons between 72 and 84 years of age with transparent lenses were obtained from the eve-bank of the Department. Fresh cataracts were obtained from cataract surgery patients. IDO activity was measured by the method of Yamazaki et al (Biochem J 1985; 230:635) adapted for HPLC. KAT activity was measured by the method of Tobes (Methods Enzymol 1987; 142:217). IDO activity found in post-mortem transparent lenses (cortex and nucleus) was $0.7 \pm 0.3 \mathrm{nmol} / \mathrm{mg}$ protein/hour. In cataracts a low IDO activity of $0.3 \pm 0.2 \mathrm{nmol} / \mathrm{mg}$ protein/hour was found in the cortex but not in the nucleus. KAT activity, found in the eight cataracts examined, was $1.6 \pm 0.9 \mathrm{nmol} / \mathrm{mg}$ protein/hour. Inhibition of IDO activity and induction of KAT activity seems to be involved in human cataract formation.

SNF 32-36058.92, EMDO, and Hartmann-Müller 


\section{PHOTOLINKER POLYMER MEDIATED IMMOBILIZATION OF MONOCLONAL ANTIBODIES AND F(AB') FRAGMENTS}

Gao H. ${ }^{1}$, Kislig E. ${ }^{1}$, Oranth $N^{2}{ }^{2}$ and Sigrist $H^{1}$

1 Institute of Biochemistry, University of Bern, Freiestrasse 3, CH-3012

Bern and 2 F. Hoffmann-La Roche Litd. CH-4002 Basel, Switzerland

Photolinker polymer mediated covaient inmobilization of antibodies and F(ab') fragments has been achieved by newly established lightdependent coupling procedures. Anti alpha-1-fetoprotein monoclonal antibodies and $F\left(a b^{\prime}\right)$ fragments derived from anti prostate specific antigen monoclonal antibodies were covalently linked to microplates. Diazirine derivatized bovine serum albumin served as multifunctional light activatable linking agent. Both immunoreagents, monoclonal antibodies and $F\left(a b^{\prime}\right)$ fragments remained immunologically active after $350 \mathrm{~nm}$ irradiation (irradiance $0.7 \mathrm{~mW} \mathrm{~cm}^{-2}$ for 20 minutes). Covalency of antibody binding was inferred from i) the photoreagent dependence, ii) the light dependence of the immobilization process, and iii) the reversibility of immunocomplexation after acid treatment.
TWO COMPONENIS OF EXIRACEILULAR ELECIRTCAL RESISTANCE IN VENIRICULAR MYOCARDIUM

Fleischhauer J.C., Kléber A.G., Dept. of Physiol. Bern In mocardial tissue, the electrical resistive properties of the extracellular space are important for local current flow during excitation and therefore influence impulse conduction and the magnitude of the extracellular electrical field. To assess the multicompartment nature of the resistance of the extracellular space, electrical cable analysis was apolied on an arterially perfused rabbit papillary muscle. The resistivity of the intravascular space was changed ( 70 to $220 \Omega \mathrm{cm}$ ) by variation of hematocrit from 0 to $60 \%$ in the perfusate. In order to change the volume and hence the electrical resistance of the interstitial space, colloidosmotic pressure in the perfusate was varied by changing dextran $\left(M_{2} 70000\right)$ concentration (10 to $80 \mathrm{~g} / 1$ ). Altering the interstitial space volume had a marked influence on the extracellular resistance $\left(x_{0}\right)$, conduction velocity and the magnitude of the extracellular field. Variations of the resistive properties of the intravascular space had no significant influence on $r_{0}$, conduction velocity and the magnitude of the extracellular electrical. field. Our results suggest that the microvascular tree is insulated from the interstitial space and local electrical current flow during excitation in heart muscle is confined to the narrow interstitial clefts.

\section{S23-32}

IN VITRO AND IN SITU PROTEOLYSIS OF SOME CYTOSKELETAL PROTEINS BY THE CB ${ }^{2+}$-DEPENDENT PROTEASE IN ALLOMYCES Huber D., Ojha M. and Turian G. Laboratory of General Microbiology, University of Geneva, Sciences IIT, 30 Quai Ennest-Ansermet, 1211 Geneva 4, Switzerland.

$\mathrm{Ca}^{2+}$-dependent protease in Allomyces is predominantly localized in the apical region of the exponentially growing hyphae (Huber and Ojha, submitted). Many cytoskeletal proteins like actin and tubulin are also mainly localized in this growing region (Heath, 1991). It is known that the $\mathrm{Ca}^{2+}$-dependent protease proteolyzes a number of cytoskeleton proteins in order to regulate the plasticity of the cell (Mellgren, 1987). We have studied the proteolysis of some cytoskeletal proteins by this protease purified from the aquatic fungus Allomyces arbuscula. Actin, tubulin and desmin, were found to be rapidly proteolyzed in vitro at a high substrate to enzyme ratio. Immunofluorescence studies of protealysis made in situ in exponentially growing hyphae showed modification of apically localized cytoskeletal proteins. This colocalization of the $\mathrm{Ca}^{2+}$-dependent protease and cytoskeletal proteins in the same apical region is inferred in relation to hyphal elongation. Heath, I.B. (1991) Tip Growth in Plant and Fungal Cells. Academic Press. Mellgren, R.L. (1987) FASEB J. 1: 110-115. This research is supported by FNRS $N^{*} 32-31997.91$ to D.H.

Bioinformatics Research for the Researchers

Swiss Node of the European Molecular Biology Network | R.Doelz and F.Eggenberger, Biocomputing, Biozenirum der Universitoz, Kunibas.ch

R.Doelz and F.Eggenberger, Biocomputing, Biozentrum der Universität, Klingelbergstrasse $70, \mathrm{CH} 4056$ Base

Biological databases grow exponentially. In order to provide access, as much dara as needed and as little volume as possible shall be returned upon a query. The currently available DNA and Protein sequence databases are updated in a currently available DNA and Protein sequence databases are updated in a
sophisticated network of procedures and expose a considerable amount of sophisticated network of procedures and expose a considerable amount of
redundancy. Research is performed on optimizing the creation of non-redundant data sets. The resulting data are distributed in Switzerland, or made accessible to researchers who cannot utilize local resources. Transparent access to the Swiss resources, as well as paneuropean services, is provided with a job scheduling system which was developed in Basel, the Hierarchical Acces System for Sequence Libraries in Europe ("HASSLE"), and various of which allow comprehensive browsing such as "GOPHER" and "WWW". Training courses to use the resource effectively are running throughout the year.

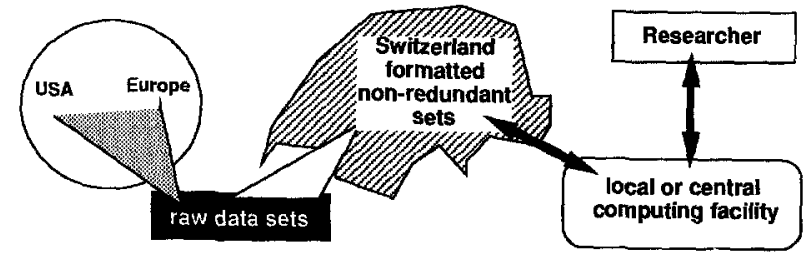


Acha-Orbea, H. S01-07

Ackermann, M. S04-25, S15-69, S20-05

Ackermann, R. S06-13

Adler, H. S05-54

Adrian, M. S01-10

Aebi, M. S15-23

Aebi, U. S10-18

Aebischer, P. S17-12

Aeschlimann, C. $\mathbf{S 2 1 - 2 0}$

Affolter, M. S19-06, S23-02

Agostini, E. S16-05

Aguet, M. S05-13, S14-14, S14-21, S14-22

Aguzzi, A. S14-21

Aguzzi, P. S03-14

Alberi, S. S12-15

Albrecht, D. S05-20, S18-10

Alliod, C. $\quad$ \$7-09

Altmann, M. S06-07

Amadò, R. S13-01

Ambs, S. S02-02

Amiguet-Barras, F. S05-29

Amrhein, N. S13-06

Anagli, J. S15-08

Andjelkovic, M. S05-44

Andres, A.-C. S05-18, S05-19, S05-20

Anjard, C. S05-40

Appel, R. S10-19

Appert, C. S13-06

Araki. K. S14-24

Arami, S. S15-28

Arber, W. S04-01, S04-09

Arcaro, A. S05-49, S05-50

Aregger, H. S14-27

Ami, S. S05-34

Arsenijevic, D. S09-04

Arsenijevic, Y. S01-08, S01-09

Ashbridge, K. S17-08

Assimacopoulos-Jeannet, F. $\quad$ S05-28

Astori, M. S01-07, S08-04

Atanasoski, S. S20-10

Aubert, J.D. S02-01

Autenried, P. S03-14

Bächi, T. S15-63

Bachmann, D. S08-04

Bachmann, F. S15-35

Bachofen, H. S02-04

Backendorf, C. S05-29

Backes, S. S18-03

Bader, C.-R. S11-05, S11-06, S12-19

Badman, G.T. S15-38

Baertsch, S. S03-09

Bähler, J. S03-04, S03-10

Bahnholzer, R. S14-31

Baici, A. S23-07
Bajo Lorenzana, V.M. S17-07, S17-10

Bakardjiev, A. S05-04

Balant, L.P.. S06-01

Balàzs, R. S12-12

Baldinger, B. $\quad \$ 22-02$, S22-04

Ball, R. S01-12

Ballèvre, L. S14-35

Ballmer-Hofer, K. S10-04, S10-06

Banach, K. S12-11

Bankoul, S. S17-15

Barakeat-Walter, I. S15-60

Barangé S. S14-13

Barja, F. S23-12

Barker, P. S05-55

Baroffio, A. S11-05, S11-06

Barrera, I. S18-01

Baschong, W. S10-18

Bashkirov, V. S03-01, S03-03

Batra, S. S02-05

Bättig, K. S22-02, S22-04

Bauer, C. S05-16

Baur, M. S03-12

Baur, R. S12-09

Bchini Hooft van

Huijsduijnen, O. S09-08

Beard, P. S04-05, S04-22

Beck, C. S14-34, S15-51, S15-66

Bednar, J. S03-17

Beer, H.F. S17-26

Beermann, F. S12-13, S14-16, S20-14

Beggah, A.T. S15-03

Beghdadi-Rais, C. S15-10

Beguin, P. \$15-03

Belet, M. S10-16, S10-19, S15-43

Benner, S. S23-14

Berchtold, M.W. S03-16, S05-21, S23-23

Berdoz, J. S08-04

Beretta, C. S14-34, S15-66

Berger, E.G. S08-12, S15-63

Bernard, M. S16-09

Bernard, V. S06-06

Bernasconi, M. S19-02

Bernheim, L. S12-19

Beron, J. S15-24

Bertoni, G. S04-20, S14-28

Bertrand, D. S12-06, S12-07

Betschart, B. S16-12

Beug, H. S08-15

Biber, J. S05-30, S15-27, S15-54

Bielke, W. S01-06, S01-12, S02-03

Bigler, D. S15-41
Bilbe, G. S18-03

Billeter, M.A. S04-03, S04-15

Billeter, R. S09-13, S09-14

Billotte, C. S05-47

Binz, P.A. S23-25

Bissell, M.J. S11-03

Blaser, K. S14-07

Blatter-Garin, M.-C. S01-05

Blau, N. S23-17

Blot, M. S04-01, S04-12

Blum, H.E. S04-16, S04-28

Blümcke, I. S17-16, S17-17

Bobbioni-Harsch, E. S09-17

Bochaton-Piallat, M.-L. S10-15

Boeck, R. S06-04

Bohlhalter, S. S12-24

Bolivar, I. S23-12

Boller, T. S14-16

Bolliger, L. S09-03

Bonnard, C. S18-05

Bootman, M. S05-43

Bordmann, G. S14-06

Borner, C. S01-17

Bornmann, C. \$20-06

Boss, O. S15-38

Bouron, A. S05-14, S12-16

Bourque, A. S23-28

Bousset, K. S05-04

Bouvier, J. S16-19

Bovia, F. S06-05

Boyan, G. S17-14

Brack, Ch. S06-12, S06-13

Braissant, O. S05-37

Brander, K. S06-02

Brandner, C. S22-01

Brcic-Kostic, K. S19-07

Breitkreutz, D. S05-29

Brenner, H.R. S12-18

Brenz Verca, S. S05-10

Bron, C. S15-10

Bruinink, A. S14-10

Brunisholz, R.A. S15-45, S15-47

Brunner, A. S01-14

Brunschwig, K. S20-03

Bucher, P. S05-23

Büeler, H. S03-14

Buetti, E. S20-08

Bugnon, O. S05-06

Bui, N. S06-11

Bukauskas, F.F. S12-10

Bulliard, C. S23-19

Burger, M.M. S05-46, S08-13, S15-35

Bürgisser, D. \$23-01

Bürglin, T.R. S20-03

Burgunder, J. S09-14

Burgunder, J.-M. S21-05
Burkhart, T. S16-07, S16-08

Bürki, K. S17-01, S17-02

Burnay, M.M. S05-07

Burri, M. S16-12

Burri, P. S02-03

Burri, P.H. S01-13

Buser, P. S06-03

Cachelin, A.B. S12-21

Calain, P. S16-17

Callaerts, P. S19-01

Canessa, C. S12-20

Cantello, B.C.C. S15-38

Cao, Y. S14-18

Capogna, M. S21-15

Capponi, A.M. S05-07, S05-12

Car, B. S14-33

Car, B.D. S14-14

Carafoli, E. S15-06, S15-07, S15-08

Cardinaux, J.-R. S21-03

Cargnello, R. S15-32, S15-33

Carlberg, C. S08-01

Carrel, S. S14-29

Carù, B. S09-16

Casperson, G.F. S11-03

Cassinotti, P. S16-20, S16-21

Cassuto, E. S03-18

Cathomen, T. S15-01

Cattaneo, R. S04-15, S15-01

Cavadore, J.-C. S10-02

Cavin, C. S20-08

Cawthorne, M.A. S15-38

Cecchini, M.G. S11-09

Celio, M.R. S10-13, S10-14, S16-11, S17-16, S17-17

Cerni, C. S05-04

Cerny, T. S21-20

Cerretelli, P. S09-16

Chamoin, M.C. S11-06

Chan, S.J. S08-04

Chanson, A. S15-17

Chaponnier, C. S23-13

Chatellard, P. S06-10

Cheevers, W.P. S04-20

Chen, P.-X. S15-30

Chicaiza, G. $\quad$ \$20-17

Chiquet, M. S11-07

Choffat, Y. S20-05

Christen, P. S23-07, S23-08, S23-09

Christoffel, M. S14-05

Cirulli, V. S23-04

Claassen, H. S09-12

Claeys, D. S15-29, S15-31

Clarke,.P.G.H S01-01, S01-04 
Clarke, S. S01-01, S17-07

Clay, O. \$20-15

Clémence, J.F. S17-12

Clever, B. S03-02

Cohn, W. S14-10

Colello, R. S15-34

Colello, R.J. S17-24

Collart, L. S21-06

Collet, Y. S13-04

Colombi, M. S08-09

Content, J. S05-45

Conzelmann, A. S15-62

Cooney, A. S19-09

Coppolecchia, R. S06-03

Corthésy, B. S08-05, S08-06, S08-08

Corthésy, P. S14-15, S14-16

Costello, E. S04-22

Cotecchia, S. \$15-55

Cottet, S. S08-06

Cox, J.A. S23-23

Croft, S.L. S16-01

Cromton, N.E.A. S08-11

Cron, P. S05-24, S05-44

Cullmann, G. S10-03

Cusin, I. S09-06, S09-07

Dammann, R. S20-01

Darby, I. S01-15

Darszon, A. S09-09

Daugeron, M.-C. S06-03

Dayer, P. S21-06, S23-21

de Agostini, A. S07-01

de Ribaupierre, F, S17-07

de Ribaupierre, Y. S09-18

de Sousa, R.C. S15-42, S15-49

de Tribolet, N. S14-20, S14-23

de Vantéry, C. S10-01

Debanne, D. S17-04

Déglon, N. S15-10, S16-09

Deiner, E. S08-15

Dellis, J. S05-45

Denzer, A.J. S12-17

Desbaillets, I. S14-20, S 14-23

Deshusses, J. S10-16, S10-19, S15-43

Desmeules, J. \$21-06

Desmouliere, A. S01-15

Desponts, C. S16-09

Desvergne, B. S05-32, S05-36

di Paolo, C. S14-26

Di Vetta, V. S09-10

Diezi, J. S23-18

Diggelmann, H. S04-13, S04-14, S04-27

Digicaylioglu, H.M. S12-22

Dinter, A. S08-12

Diserens, A.-C. S14-20, S14-23

Do, K.Q. S21-16

Dobbelaere, D. S16-18

Döbbeling, U. S03-16
Doelz, R. S24-01

Dolivo, M. S15-11

Dollenmaier, G. S16-20

Domanska-Janik, $\mathrm{K}$. S15-11

Dörig, R.E. S15-41

Douville, P. S04-17, S04-23

Dowling, P. S04-03

Doyle, P. S17-13

Drees, N. S14-06

Dreifuss, J.J. S01-08, S01-09, S12-14, S12-15

Drenhaus, U. S17-22

Dreyer, J.-L. S15-67, S23-19, S23-20

Driscoll, P. \$22-03

Droz, B. S15-60

Dubochet, J. S03-17

Dubois-Dauphin, $M$. S01-03, S12-15

Duc, C. $\quad$ \$17-18

Duda, P. S18-08

Dudler, U. S10-08

Dulloo, A.G. S09-04

Dunderdale, H. S03-17

Dunkel, M.P.H. S13-01

Duport, S. S17-23

Durand, J. S05-41, S05-42

Durussel, I. S23-23

Dusart, I. S21-07

Dutler, H. S23-14

Dykeman, A. S23-28

Eberhard, J. S13-02

Eberhardt, W. S14-02, S14-12

Eder-Colli, L. S15-40

Egelman, E.H. S03-17

Eggenberger, F. S24-01

Egger, M. S12-23

Eggli, S. S17-16

Ehrlich, D. S03-18

Eichele G, \$19-09

Eichhorn, M. S16-18

Eldering, J.A. S15-59

Elke, C. S05-48

Ellenrieder, C. S10-17

Eng, V.M. S14-14

Engel, J. S15-37, S15-39

Engels, M. S15-69

Eppenberger, H.M. S11-04, S15-12

Eppenberger, U. S14-08

Erard, F. S14-07

Erne, B. S15-46

Erny, K. S04-13

Erulkar, S. S17-05

Escorihuela, R.M. S22-03

Espel, E. S05-23, S14-17

Etter, A. S06-06

Eugster, H.P. S14-27

Falke, D. S15-64

Faller, P. S21-08

Farinelli, C.C.J. S09-16

Fasciati, R. S14-25
Fasel, N. S15-10, S16-09, S16-16

Favre, D. S06-09

Favre, I. S12-03

Felix, R. S11-09

Fellay, B. \$16-11

Felleisen, R. S20-04

Feller, U. S13-05

Felley-Bosco, E. S02-02

Fernandez, A. \$10-07

Fernández-Teruel, A. S22-03

Ferracin, F. S10-21

Ferrannini, E. S09-06

Ferrari, E. S10-10

Ferretti, G. S09-16

Fichtel, A. S04-04

Fien, K. S10-03

Fischer, A. S13-05

Fischer, M. S03-14

Fivaz, J. S13-14

Fleck, O. S03-11

Fleisch, H. S11-09

Fleischhauer, J.C. S23-33

Fleming, A.J. S13-03

Flook, P.K. S04-02

Floth, C. \$10-10

Flück, M. S05-19

Flury, N. S14-08

Fogh, J. S15-56

Foletti, D. S15-07

Fontana, A. S20-10

Forgo, J. S05-30

Forrer, P. S10-17

Foster, W. S23-28

Fraefel, C. S20-05

Frankhauser, C. S10-05

Frankowsky, H. S01-03

Frech, B. S03-19

Frei, E. \$19-07

Freiburghaus, A.U. S15-53

French, L. S01-11

Freydl, E. S13-16

Fries, R. S08-14, S20-10

Friis, R. S01-06, S01-12, $\mathrm{S} 02-03$

Fritschy, J.M. S12-24

Früh, F. S06-14

Fruitiger, S. S09-17

Fucentese, M. S15-27

Fuchs, R. S04-27

Fundel, S. S21-08, S23-26

Furger, A. S18-10

Furter, R. S15-12

Gabbiani, F. S10-15, S23-13

Gabbiani, G. S01-15, S10-15, S23-13

Gabriel, J.-M. S15-46

Gadient, R.A. S14-03

Gähwiler, B.H. S05-02, S05-05, S17-04, S2 I-15

Gallant, P. S01-18

Gallati, H. S14-06

Gamboni, S. S05-40
Gander, J.-Ch. S10-14

Ganten, D. S09-06

Gao, H. \$23-31

Garcia, I. S14-24

Garcia-Sanz, J.A. S14-04

Garcin, D. S04-06

Gasser, D. S21-17

Gavin, A.-C. S10-01, $\mathrm{S} 10-02$

Gaymard, E. S23-22

Geering K. S15-03, S15-29, S15-30, S15-31

Gehring, H. S23-07, S23-08

Gehring, W. S19-01

Gehring, W.J. S19-03, S19-04, S19-05, S19-06

Gellissen, G. S04-02

Genini, M. S08-14

Georgaki, A. S10-10, S23-05

Georgiev, O. S04-17, S05-13, S20-16

Gerber, U. S05-02, S05-05

Gerber-Bologa, L. S17-11

Gerster, T. \$20-06

Gesemann, M. S05-01, S12-17

Giacobino, J.P. S15-38

Girardier, L. S09-04, S09-16

Girod, P.-A. S13-15

Gitler, C. S05-46

Givel, F. S08-01

Glardon, S. S19-01

Glaser, T.A. S16-09, S16-16

Glick, B.S. S09-01, S09-02, S09-03

Goedert, M. S17-01

Goethals, M. S23-13

Goetschy, J.F. S05-45

Golaz, O. S06-01

Goldmann, W.H. S15-25

Gosert, R. S16-21, S16-22

Gottesman, M.M. S23-16

Gottstein, B. S16-02, S16-15, S20-04

Götz, J. S17-01

Gotzos, V. S10-13, S10-14

Gouyon, J.-B. S14-36

Graber, M. S21-05

Graber, R. S01-02

Grau, G. S14-24

Gravel, P. S06-01

Greber, U.F, S04-08

Greeff, N.G. S12-22, S12-23

Greipl, R. S15-28

Gröning, K. S18-02

Groppe, J. S19-06

Gross, N. S14-34, S15-66

Grossniklaus, U. S05-44

Grosso, A. S15-42

Groux, D. S05-40

Grun, H. S14-10

Grünberg, J. S15-59 
Grünberger, S. S15-17

Gruszecki, W.I. S15-65

Grüter, P. S18-04

Gschwind, M. S03-15

Gstaiger, M. S04-29, S05-53

Guérineau, N.C. S05-02

Guerini, D. S15-06, S15-07

Gueroguiev, O. S04-23

Gugerli, P. S13-11

Guignard, F. S05-15

Guignard, J.-P. S14-35, S14-36

Guillaume-Gentil, C. S05-28, S17-13

Guisse, B. S13-10

Gunten, A.v. S17-22

Guo, K. S01-06, S01-12

Gyger, U. S02-04

Haenni, A.-L. S04-06, S04-07

Hagenbuch, B. S15-48, S15-52

Hagenbüchle, O. \$20-09

Hagmann, M. S04-17, S04-23

Hahn, S. S14-31

Hahne, K. S15-04

Halban, P. S23-04

Hale, T.I. S23-09, S23-25

Halter, D. S20-12

Hammann, M. S11-06

Hamou, M.-F. S14-23

Hamou, M.-H. S14-20

Hanke, W. S14-37

Harpur, A S05-18

Harris, C.C. $\mathrm{S} 02-02$

Hartmann, F. S14-29

Hartsuiker, E. S03-10

Hasenfratz, M. S22-02, S22-04

Haugg, M. \$23-10

Hauke, H. S15-51

Hauptmann, G. S20-06

Hausel, P. S23-18

Häuselmann, R. S12-04

Hauser, N. S11-02

Haussmann, I. S18-06

Haustein, A. S04-32

Hawkes, R. S17-20

Hayashi, S. S02-01

Hayes, G. S05-30

Hecht, P. S15-69

Hecker, H. S16-12

Hefti, F.F. S15-61

Hefti, H.P. S14-26

Heider, H. S05-08

Heizmann, C.W. S23-01, S23-17

Helenius, A. S04-08

Hemmings, B.A. S05-24, S05-44, S10-07, S10-20

Hemphill, A. S16-01

Henderson, B.R. S18-05

Henriksson, M. S05-04
Henry, H. S13-04

Herczeg, A. S12-18

Hernandez Torres, J. S06-10

Herrenknecht, K. S15-37

Herrling, P.L. S12-27

Herrmann, A. S04-31

Hertel, C. S05-55

Hertig, C. S04-19

Hess, M.W. S14-01

Heuchel, R. S05-13

Heyer, W.-D. S03-01, S03-02, S03-03, S03-08

Hihi, A.K. S05-38

Hildbrand, M. S13-05

Hill, F. S16-05

Hille, A. S15-63

Hillenbrand, R. S15-50

Hindges, R. S10-08

Hindley, R.M. S15-38

Hinz, U. S13-12, S13-14

Hirsch, H.H. S14-32

Hirt, B. S04-22

Hobi, R. S03-16

Hochstrasser, D. S10-16, S10-19

Hochstrasser, D.F. S06-01

Hoessli, D.C. S05-34

Hofer, R. S13-04

Höfferer, M. S05-09

Hofstetter, W. S11-09

Hogg, J.C. S02-01

Hohl, D. S05-29

Holm, J. S15-50

Holz, A. S17-24

Honegger, P. S10-21, S17-21, S20-18, S21-02

Honegger, U.E. S21-09, S23-03

Hopkins, S.A. S01-07

Hoppeler, H. S09-12, S09-13, S09-14

Horisberger, J.-D. S15-18

Horisberger, M.A. S05-45

Hornung, J.P. S21-04

Hosseini, G. S07-01

Hossle, J.-P. S23-06

Hottiger, M. S04-11

Hovens, C. S05-53

Hu, H.-Y. S12-07

Huang, S. S14-14

Huang, Y.-J. S16-04

Huarte, J. S01-03

Huber, D. S23-32

Huber, G. S03-15

Huber, M. S05-29

Hübscher, U. S04-11, S10-08, S10-09, S10-10, S23-05

Hug, M. S05-09

Hughes, G. S09-17

Hummler, E. S12-13

Hunger, R.E. S14-01

Hunziker, P.E. S06-08

Hunziker, T. S14-19

Hurer, H. S05-30
Hurt, N. S14-06

Huwiler, A. S05-25

IJpenberg, A. S05-32

Imhof, B.A. S16-10

Imhof, M.O. S05-51

Inderbitzin, S. S21-01

Interthal, $\mathrm{H}$. S03-01

Irmler, M. S01-17

Isenberg, G. S15-25

Iwata, T. S15-06

Jackson, D. S15-66

Jackson, P.K. S09-08

Jaggi, R. S01-16, S20-17

Jaisser, F. S15-03

James, R.W. S01-05

Jans, D.A. S05-03

Jans, P. S05-03

Janzer, R.C. S14-09

Jaquet, P. S15-49

Jaunin, F. S13-04

Jaussi, R. S10-17

Jeannin, E. S05-36

Jeanrenaud, B. S05-28, S09-06, S09-07, S09-17, S17-13

Jehn, B. S01-16, S20-17

Jensen, M. S15-56

Jentsch, S. S16-03

Jéquier, E. S09-10

Jessberger, R. S03-06

Jin, Y. S07-02

Jirounek, P. S21-19

Joho, R.H. S12-01, S12-02

Joiner, M. S08-11

Jostarndt, K. S09-13, S09-14

Jucker, M. S15-28

Juillerat-Jeanneret, L. S14-09

Jung, H. S05-16

Jungi, T.W. S05-54

Junne, T. S09-02

Kadaré, G. S04-07

Kaelin, A. S17-11

Kaelin, K. S04-03, S04-15

Kägi, J.H.R. S23-24, S23-25

Kahle, P. S05-55

Kaldis, Ph. S15-12

Kaldy, P. S18-11

Kalousek, M. S05-39

Kammer, A. S16-07

Kapfhammer, J.P. S21-07

Kappos, L. S10-21

Karapetian, O. S01-10

Karapetin, O. S01-07

Kasas, S. S15-32, S15-33, S16-11, S16-14, S17-16

Käslin, E. S03-08

Kasper, P. S23-08

Katela, E.F. S04-32

Kater, S.B. S05-11

Kato, A. S01-05
Kaufmann, S. S15-25

Keegan, L. S19-03

Keist, R. SO4-16

Kellenberger, S. S12-09

Keller, H. S08-01

Keller, H.U. S11-08

Keller, W. S18-07

Keppler, D. S08-04

Kernen, P. S09-09, S15-65

Kesselring, F. S05-22

Kessler, R. S05-39

Kester, H. S03-01

Kinne, R.K.H. S15-54

Kiraly, M. S21-14

Kisling, E. S23-31

Kléber, A.G. S23-33

Klemenz, R. S05-39

Kloter, U. S19-05, S19-06

Koch, M. S11-07

Koedood, M. S04-04

Köhler, G. S15-02

Kohler, R. S18-08

Kohli, J. S03-04, S03-07, S03-10, S03-11, S03-12, S03-13

Kolakofsky, D. S04-06, S06-04

Koller, T. S20-01

König, M.F. S02-05

Kopp, M. S08-07

Korte, T. S04-31

Kosovsky, J. S10-17

Kraehenbuhl, J.-P. S01-07, S15-29, S15-31

Kraftsik, R. S17-07

Krämer, A. S18-02, S18-03, S18-04

Krapp, A. S20-09

Krause, R.M. S12-19

Kreis, T.E. S10-12

Krstic, R. S21-13

Krucker, T. S12-27

Krummenacher, C. S04-14

Krupa, Z. S15-65

Kubli, E. S15-41

Kucera, P. S09-18

Kuenzle, C.C. S03-16

Kuhlemeier, C. S06-02, S13-03

Kühn, L.C. S18-05, S18-11

Kuhn, T.B. S05-11

Küng, M. S23-27

Kunz, C. S04-03

Kunz, D. S14-02, S14-12

Küpfer, A. S21-20

Kurasaki, M. S23-24

Kuratani, S. S19-09

Kürz, L.L. S12-02

Laban, O.P. S05-12

Lahm, H. S14-33

Laissue, J.A. S14-01

Lamb, N.J.C. $\quad$ S10-07

Lambin, P. S08-11

Landis, H. S14-26 
Landmann, L. S15-20, S15-52

Lange, J. S13-17

Lanker, S. S06-07

Lanzrein, B. S16-13

Larkum, M.E. S17-06

LaRosa, M. S19-06

Larsson, B. S08-11

Larsson, L.-G. S05-04

Lassmann, H. S10-21

Lattion, A.L. S15-55

Laurent, J.P. S15-09

Laurent, R. S16-17

Le Marchand-Brustel, Y. S05-28

Lechner, F. S04-24

Ledermann, B. S17-02

Leemann, T. S23-21

Lehmann, R.P. S15-47

Lehnert, M. S08-03

Leighton, J. S15-05

Leimbacher, W. S23-17

Lemberger, T. S05-37

Lengweiler, S. S15-22

Lenig, D. S14-04

Leoni, L. S15-13

Lesslauer, W. S14-24

Lezzi, M. S05-48, S05-51

Li, X. S19-08

Lichtensteiger, W. S12-26, S17-26, S21-01

Lichtsteiner, M. \$12-18

Limat, A、 S14-19

Linder P. S06-03

Lindner, H. S16-12

Lingen, M. S15-64

Lipp, P. S05-26, S05-27

Liu, W. S14-18

Livingstone-Zatchej, $\mathrm{M}$. S20-12

Lo Russo, A. S23-11

Loepfe, E. S15-69

Loosli, F. S19-01

Lorenzoni, P. S08-13

Losa, G.A. S01-02, S15-13

Louvion, J.-F, S05-35

Lowenstein, C.J. $\quad \mathrm{SO2-02}$

Lowin, B. S01-11

Lucchini, R. S20-01

Lucocq, J.M. S05-08

Ludin, B. S17-08

Ludwig, C.U. S08-09, S08-10

Ludwig, S. S04-32

Lührmann, R. S18-06

Lüscher, B. S05-04, S12-04

Lüscher, C. S17-03

Lïscher, H.-R. S17-03, S17-06

Lïscher-Firzlaff, J.M. S05-04

Lüthi, A. S15-09

Luithi, D. S12-05

Luthi, F. S04-13

Lutz, B. S19-09

Lutziger, I. \$06-02
Maake, C. S14-37

MacDonald, H.R. S14-16

Mackay, E.A. S23-24

MacMahon, A. S23-28

Maeder, B. S23-12

Maffeis, C. S09-15

Magistretti, P.J. S15-15, S15-16, S21-03, S21-11, S21-12, S21-14, S21-16, \$21-17

Magnenat, L. S16-06

Maier, A. S17-26

Maier, D. S05-31

Maier, P. S14-05, S14-25

Malaise, E. S08-11

Malherbe, P. S12-09

Malina, H. S23-29, S23-30

Malipiero, U. S20-10

Mandel, T. S06-02

Marc-Martin, S. S13-11, S23-22

Marchal, G. S14-24

Marconi, C. S09-16

Marguerat, A. S01-09

Markert, M. S05-15

Marmy, N. S05-41, S05-42

Marti, A. S01-16

Marti, D. S12-08

Marti, H. S05-16

Martin, F. S20-17

Martin, J.-L. S15-16, S21-17

Martin, X. S23-29, S23-30

Martinez, R. S15-14

Martinou, I. S01-17

Martinou, J.-C. S01-17, S01-03

Masson, D. S10-12

Masternak, K. S04-26

Mathews, P.M. S15-29, S15-30, S15-31

Mathis, N. S15-38

Matile, H. S16-10

Matsuo, K. S20-15, S20-16

Mattenberger, L. S01-05

Matthieu, J.-M. S15-11, \$20-18

Matthisson, M. \$11-07

Mattioni, T. S09-08

Mattmann, C. S01-17

Matus, A. S17-08

Mauël, J. S05-15, S16-09, S16-16

Maurer, H. S15-27

Maury, K. S12-06

Mayer-Jaekel, R.E. S10-20

McGuigan, J.A.S. S12-05

McHenry, C. S04-11

Meda, P. S12-11

Medilanski, J. S15-40

Medin, J. S05-38

Meier, C.L. $\quad$ S12-27

Meier, J. S04-12

Meier, P. S04-04

Meier, P.J. S15-48, S15-52

Meili, R. S10-06
Menger, A. S15-17

Menotti, E. S18-05

Mermod, N. S05-38

Merz, V. S01-12

Messerli, J.M. S11-04

Messmer, S. S01-05

Métraux, J.-P. S13-07,S13-08, S13-09

Metzger, D. S12-14

Meuwly, P. S13-07, S13-08, S13-09

Meyer, J. S04-10, S20-13

Meyer, U.A. S06-14

Meyer-Monard, S. S14-30

Meylan, M. S23-15

Michel, M.R. S06-09

Miescher, G.C. S10-21, S15-46

Miklossy, J. S16-14

Millward, T. S05-24

Milon, G. S14-24

Mitchell, P.J. S04-04

Miyasaki, Y. S14-24

Mock, B. S12-22

Möhler, H. S12-12, S12-24

Mohr, U. S13-17

Mölders, W. S13-08, S13-09

Molinari, M. S15-08

Monnet-Tschudi, F. S20-18, S21-02

Montag, D. \$15-50

Montagne, J. S19-06

Moos, M. S15-50

Morel, P. S03-18

Moroni, C. S14-30, S14-31, S14-32

Morris, H. S09-17

Moser, M. S15-34

Motejlek, K. S12-04

Moulet, O. S15-67

Mrkoci, K. S08-12

Mueller, C. S14-01

Mueller, H. S14-08

Mueller, R. S10-17

Mühl, H. S14-11

Mühlebach, S. S19-02, S23-03

Müller, B. S15-39

Müller, F. S06-06, S16-03, S16-04, S16-05, S16-06, S20-03, S20-11

Müller, L. S13-12

Müller, N. S16-02

Muiller, P.P. S06-07

Munoz, M. S15-14

Murata, J.I. S14-09

Murer, H. S15-54

Muslema, N. S10-19

Muster, L. S03-05

Muzzin, P. S15-38

Naas, T. S04-12

Nabholz, M. S05-23, S14-13, S14-15, S14-16, S14-17
Naim, H.Y. S15-19, S15-21

Nair, A.P.K. S14-31, S14-32

Nakagawa, J. S14-30

Nakano, M. S05-17

Nar, H. S23-01

Nazeri, M. S10-16, S15-43

Neely, M.D. S05-01

Neerman-Arbez, M. S23-04

Neuhaus, J-M. S13-16

Nicholls, J. S17-05

Nicholls, J.G. S05-01

Nichols, A. S03-05

Nickelsen, J. S18-09

Nicolas, D. S21-13

Niederöst, B. SO4-16

Nielsen, P.J. S15-02

Nigg, E.A. S01-18

Niggli, E. S05-26, S05-27

Niggli, V. S15-25

Nilsen, T. S16-06

Nilsson, P, S15-56

Noll, M. S19-07, S19-08

Noll, T. S16-09

Novier, A. \$21-13

O'Connell, M.A. S18-07

Oesch, B. S15-34, S15-56

Offord, E. S04-05

Oft, M. S08-15

Ohno, K. S14-25

Ojha, M. S23-32

Oranth, N. S23-31

Oropesa, C. S17-23

Otten, U. S14-03

Owttrim, G. S06-02

Ozato, K. S05-38

Ozmen, L. S14-14

Palmer, G. S05-35

Pancino, G. S04-20

Panico, M. S09-17

Panzer, P. S15-21

Papadopoulos, D. S04-01

Paré, P.D. S02-01

Parisi, L. S17-23

Parisi, S. S03-13

Passaquin, A.C. S23-11

Pauli, U. S14-28

Pauls, T.L. S23-23

Paulsson, M. S11-02

Pavlovic, J. S06-09, S14-26

Péclard, R. S14-05

Peitsch, M. S01-18, S15-14, S18-11

Peli, J. S08-15

Pellegri, G. S15-16

Pellegrinelli, N. S15-40

Pellerin, L. S15-15

Pèrez, L. S10-04

Perriard, J.-C. S11-04, S19-02

Perroud, M. S08-01

Perruisseau, G. S14-34

Peruisseau, G. S15-66

Peterhans, E. S03-19, 
S04-19, S04-20, S04-24, S05-54, S14-28

Peveri, P. S04-24

Pfarr, S. S10-21

Pfeilschifter, J. S05-16, S05-25, S14-02, S14-11, S14-12

Philipp, H. S04-31

Picard, D. S05-35, S09-08

Pierres, M. S05-34

Piquet, V. S21-06

Pithon, E. S17-21

Pla, M. S14-17

Podust, V.N. S04-11, S10-10

Poincelet, M. S05-34

Pokutta, S. S15-37

Pollack, I. S01-14

Pometta, D. S01-05

Portalier, P. S11-06

Porzig, H. S05-22

Pott, U. S15-34, S17-24

Pouly, S. S20-18

Pountney, D.L. S21-08, S23-26

Pralong, E. S21-14

Preiss, A. S05-31

Primi, M.-P. S01-04Prives, C. $\mathbf{S 1 0 - 1 0}$

Probst, A. S17-01

Pult, I. S04-16

Puntschart, A. S09-13, S09-14

Puoti, A. S12-20

Python, C.P. S05-07, S05-12

Quadroni, R. S17-03

Quiring, R. S19-01, S19-05

Raddatz, E. S09-18

Radecke, F. S04-03

Radtke, F. S05-13

Rager, G. S17-22

Raggenbass, M. S12-14, S12-15

Rahner, C. $\quad$ S15-20

Ramage, L. S15-04

Ramel, M.-E. S13-11

Ramirez, B.-C. S04-06

Ranieri, J.P. S17-12

Räschle, M. S10-17

Reber, B. S05-14

Reber, B.F.X. S12-16

Redard, M. S01-15

Reichenbach, P. S05-23, S14-15

Reichert, H. S17-14

Reichmann, E. S08-15

Reid, H. S05-20

Reinecke, M. S14-37, S21-10

Reis, L. S14-21, S14-22

Rentsch, J.M. S05-21

Resink, A. S12-12

Reszel, B. S20-12
Reuter, H. S05-14, S12-16

Revelli, J.P. S15-38

Reymond, C.D. S05-40

Reynaud, S. S05-33

Richard, C. S13-15

Richter, H. S13-04

Rickli, E.E. S12-08, S15-22, S23-02

Riederer, B.M. S17-20, S17-21

Rigoni, P. S20-02

Rimoldi, D. S14-29

Rindisbacher, L. S08-05, S08-06, S08-08

Riniker, F. S16-08

Rioult, M.G. S17-06

Riwar, B. S03-06

Robert, A. S21-19

Robyr, D.C. S16-16

Roch, J.-M. S15-57

Rochaix, J.-D. S18-09

Rochat, A.-C. S09-18

Rodewald, H.-R. S03-06

Rohner-Jeanrenaud, F. S09-06, S09-07, S17-13

Rohr, S. S11-01

Rohrbach, K. S14-28

Romero, P. S14-13

Rospert, S. S09-02

Ross, C.A. $\quad$ \$16-01

Rossier, B. S12-13, S12-20

Rossier, B.C. S15-03, S15-29, S15-30, S15-31

Rossier, C. S15-16

Rossier, M.F. S05-07, S05-12

Roth, F. S16-08

Roth, M.G. S15-19

Rouiller, E. S17-07

Rowell, C.H.F. S04-02

Rozanov, M. S04-07

Rubin, B.P. S21-07

Ruchti, F. S06-07

Rueda-Maza, Cl.M. S09-15

Rüegg, M.A. S12-17, S12-18

Rüegg, U.T. S12-25, S23-11

Ruff, G. S04-20

Ruffner, H. S14-22

Rülicke, T. S03-14

Rungger, D. S03-05

Rungger-Brändle, $\mathrm{E}$. S03-05, S17-09

Rusciano, D. S08-13

Rusconi, S. S05-09, S05-10, S05-51, S20-02, S23-06

Rust, G. S12-21

Rusterholz, C. S05-23

Rutishauser, B.M. S11-04

Ryffel, B. S05-52, S14-05, S14-14, S14-25, S14-27, S14-33

Saad, B. S14-05, S14-25
Sagot, Y. S01-05

Sahli, R. SO4-22

Sailer, A. S03-14

Saitoh, T. S15-57

Sakanari, J. S16-19

Salem, N. S15-40

Salili, A.R. $\quad$ S12-26

Salvi, S. S14-29

Salzberg, B.M. S11-01

Sanchez, J.-C. S10-16, S10-19

Sandmeier, E. S23-09

Sandmeier, H. S04-10, S04-21

Saurer, S. S01-06, S01-12

Sautel, M. S15-14

Schaad, N.C. S05-06

Schachner, M. S15-09, S15-28, S15-50

Schaefer, R. S14-21

Schaefer, T. S05-46

Schaeren-Wiemers, N. S15-68, S17-24

Schaerer, E. S01-10

Schäfer, B.W. S08-14, S19-02

Schaffner, W. S04-17, S04-23, S04-29, S05-13, S05-53, S20-15, S20-16, S23-06

Schaller, D. S10-16, S10-19, S15-43

Schaller, J. S12-08, S15-22, S16-12, S23-02

Schär, P. S03-11, S03-12

Schärrer, E. S01-17

Schatz, G. S09-01, S09-02, S09-03, S15-04, S15-05, S23-16

Schawalder, H.P. S14-05

Scheidegger, S. S14-08

Schein, C.H. S23-10

Schenk, F. S22-01

Scherthan, H. S03-04

Schild, L. S12-03

Schittny, J.C. S01-13, S21-09

Schlimme, W. S16-12

Schlumpf, M. S12-26, S17-26, S21-01

Schmid, D. S23-07

Schmid, J. S13-02, S13-06

Schmidhauser, C. S11-03

Schmidt, A. S20-14

Schmidt, C. S04-09

Schmidt, M.F. S05-11

Schmidt, M.F.G. S04-31

Schmidt, S. S10-05

Schmilinsky Fluri, G. S21-18

Schmitz, N. S10-11

Schmuckli, J. S03-02

Schmutz, C. S04-18

Schnebli, H.P. S07-02

Schneeberger, E. S12-03

Schneider, E. S08-03
Schneider, H. S04-03

Schneider, S. S05-10

Schneider, S.D. S23-06

Schneiter, Ph. S09-10

Schneuwly, S. S01-14

Schnyder, B. S14-14, S14-33

Scholtissek, C. S04-32

Schopfer, K. S16-07

Schopfer, K. S16-07, S16-08

Schorderet, M. S05-06

Schorderet-Slatkine, S. S10-01, S10-02

Schraml, P. S08-09

Schreiber, E. S20-10

Schreyer, M. S14-29

Schröter, M. S01-18

Schubiber, P.A. S17-26

Schultz, J. S21-04

Schümperli, D. S16-13, S18-06, S18-08, S18-10

Schuppli, D. S18-01

Schürmann, P. S23-22

Schutz, Y. S09-15

Schwab, M.E. S15-68, S17-24, S21-07

Schwaller, B. S10-13, S10-14

Schwendener, S. S15-41

Schwyzer, M. S20-05

Seck, T. S15-64

Semama, D. S14-36

Senaldi, G. S05-34

Sengstag, C.H. S03-09

Shao, Y. S08-03

Shikama, N. S06-12, S06-13

Shipman, R. S08-09, S08-10

Shooter, E. S05-55

Sidhu, M.S S04-03

Sidler, C. S14-10

Sidler, W.A. S15-44

Siegenthaler, P.A. S23-15

Sigel, E. S12-09

Sigrist, H. S17-12, S23-27, S23-31

Silke, J. S20-16

Simanis, V. S10-11

Simon, H.-U. S14-07

Singh, A. S23-28

Sipos, G. S15-62

Skutella, M. S12-25

Smith, T. S14-06

Snyder, S.H. S02-02

Sogo, J.M. S18-01, S20-01

Söhndel, S. S23-02

Soldaini, E. S14-16

Solinas Toldo, S. S08-14, \$20-10

Solinger, J. S03-01

Solletti, J.-M. S15-32, S15-33

Sonigo, P. S04-20, S04-30

Sordat, B. S08-04 
Sorg, O. S21-12, S21-16

Soriani, M. S15-53

Spaniol, P. S20-06

Sperisen, P. S14-15

Spicher, K. S05-22

Spielhofer, P. S04-03, S04-15

Spielmann, A. S13-11, S23-22

Spiess, M. S15-36

Spohr, G. S05-33

Spörri, B. S14-19

Sprumont, P. S17-11

Spycher, C. S18-10

Srivastava, A. S13-13

Stagljar, I. S15-23

Stalder H. S03-19, S04-19

Stamminger, T. S04-29

Stark, G. S05-13

Stasiak, A. S03-17, S03-18

Stauffer, Y. S04-05

Steck, A.J. S10-21, S15-46

Stefanovic, B. S18-06

Steinmann, U, S03-01

Stella, N. S21-11

Sterchi, E. S15-58, S15-59

Sterk, M. S23-08

Stettler, P. S16-13

Steuber, V. S15-50

Sticher, L. . S13-07

Stidwill, R.P. S05-17

Stieger, B. S15-52

Stieger, P. S13-05

Stillman, B. S10-03

Stoppini, L. S17-23

Stotz, A. S06-03

Strasser, R.J. S09-09, S13-10, S13-13, S15-65

Streit, A. S18-10

Streit, J. S17-03, S17-25

Strub, K. S06-05, S06-11

Stucki, J.W. S09-05

Studer, R. S06-08

Sturzenegger, B. S23-05

Stutz, E. S06-10, S13-11, S23-22

Su, Q. S05-52

Suda, K. S23-16

Suter, B. S14-28, S15-41

Sütterlin, R. S10-18

Suzuki, C.K. S23-16

Tada, M. \$14-20, S14-23

Tanaka, S. S20-12

Tangemann, K. S15-39

Tanner, M. S14-06

Tappy, L. S09-10

Taylor, V. S10-21

te Heesen, S. S15-23

Ternaux, J.P. S11-06

Thein, M. S14-06

Therianos, S. S17-14

Thimmig, R.L. S04-11

Thoma, F. S20-12

Thompson, S.M. S17-04, S21-15
Thompson-Fontaine, A. S05-31

Thonney, M. S14-35

Thöny, B. S23-17

Thöny-Meyer, L. S15-51

Thurnher, M. S08-12

Tobeña, A. S22-03

Tobler, H. S06-06, S16-03, S16-04, S16-05, S16-06, S20-03, S20-11

Tobler, K. S04-25

Tosic, M. S15-11

Touri, F. . \$17-20

Trachsel, H. S06-07

Tran, L. S09-18

Transon, C. S23-21

Tribollet, E. S01-08, S01-09

Trüb, T. S05-39

Trueb, B. S08-07, S20-07

Truttmann, A. S12-05

Trzeciak, A. S05-55

Tsai, M. S19-09

Tsaneva, I.R. S03-17

Tschopp, J. S01-10, S01-11, S01-18, S01-17

Turian, G. S23-12, S23-32

Turler, H. S06-01

Turowski, P. S10-07

Tuteja, N. S23-05

Twardzik, T. S01-14

Twentyman, P.R. S08-03

Ubl, J.J. S09-05

Udem, S.A. S04-03

Ulrich, J. S15-46

Urich, M. S10-04

Vaglio, M. S05-15

Valiunas, V. S21-18

Vallan, C. S02-03

Vallotton, M.B. S05-07, S05-12

van Berchem, S. S15-16

van Dillewyn, J. S18-09

Van Meir, E. S14-20, S14-23

Vandekerckhove, J. S23-13

Varga, Z. S17-05

Vasák, M. S21-08, S23-26

Vassalli, J.D. S10-01

Vassalli, P. S14-24

Vaughan, L. S15-26

Veit, M. S04-31

Verdumo, C. S12-13

Verrey, F. S15-24

Vesin, M.F. S05-47

Vettor, R. S09-06, S09-07

Viesturs, S. S10-05

Vilei, E.M. S15-08

Villa, A.E.P. $\quad$ S17-10

Villa, M. S12-12

Vischer, H.A. S17-19

Vital, C. S15-46

Vock, R. S09-12

Vogel, A. $\$ 20-06$
Vögeli-Lange, R. S13-17

Vogt Weisenhorn, D.M.

S17-17

Vogt, H.R. S04-20

Vögtli, M. S05-48

Vögtli, M. S05-51

von Weizsäcker, F. S04-28

Wachter, C. S09-01

Wahlberg, J.M. S15-36

Wahli, W. S05-32, S05-37, S05-38, S08-01

Waldner, H.-P. S14-30

Walker, G. S14-12

Walker, Ph. S15-14

Walldorf, U. S19-04, S19-05, S19-06

Wallimann, T. S15-12

Wallrapp, Ch. S01-14

Walter, E S04-16

Walther, P. S11-04

Wälti, E.R. S14-19

Walzer, C. S06-01

Wang, N. S23-16

Wang, S.-M. S14-15

Wang, $X$. S15-18

Wang, Y. S23-24

Weber, H. S18-01

Weber, L. S05-52

Weber, M. S14-14

Weber, P. S15-26

Wechsler, D. S21-09

Weibel, E.R. S02-04, S02-05, S09-12

Weingart, R. S12-10, S12-11, S21-18

Weise, K. S15-64

Weisenhorn, A.L. S15-33

Weiser, H. \$14-10

Weiss, N. S14-06, S16-10

Weisshaar, B. S17-08

Weissmann, C. S03-14, S14-21, S 14-22

Weitz, M. S16-20, S16-21, S16-22

Wellauer, P.K. S20-09

Welzl, H. S15-28

Wenger, R.H. S15-02

Wera, S. S10-20

Werner, A. S15-54

Weruaga Prieto, E. S17-16

Weruaga, E. S17-16

Weskamp, G. S05-55

West, S.C. S03-17

Wetterwald, A. S11-09

Wicky, C. S20-11

Widmer, F. S13-04

Widmer, H.R. S15-61

Wiedemann, C. S05-46

Wieland, S. S04-16

Wieland, S. S04-28

Wiesmann, U.N. S14-18, S14-19

Wilks, A. S05-18

Willcocks, K. S15-38

Willi, K. S04-10
Willimann, K. S16-10

Willimann, T. S08-07, S20-07

Winterhalter, K.H. S15-26

Wirth, U.V. S08-02

Wirth-Bronkowska, L.E. S08-02

Wittek, R. S04-18, S04-26, S08-05, S08-06, S08-08

Wittmann, C. S16-05, S20-03

Wittmer, B. S06-07

Wolfensberger, M. S21-10

Wong, A. S07-02

Wu, D. S02-04

Wuergler, F. S03-09

Wuethrich, G. S23-12

Wüthrich, M. S15-58

Wyder, L. S15-23

Wyler, B. S08-03

Wymann, D. S15-22

Wymann, M.P. S05-49

Wymann, M.P. S05-50

Xu, Y.N. S23-15

Xue, L. S19-08

Yang, J. S09-11

Yang, Y.L. S14-21

Yilmaz, A. S14-33

Yousefi, S. S14-07

Yu, X. S03-17

Zaffanello, M. S09-15

Zahn-Zabal, M. S03-07

Zahno, M.-L. SO4-20

Zaiko, M. S13-14

Zanger, U. S06-14

Zanoni, R. S04-20

Zarjevski, N. S09-07

Zeng, J. S20-05

Zenke, G. S05-52

Zhang, H. SO5-33

Zhang, H.-D S12-01

Zhang, H.-J. S12-02

Zhang, W. S12-05

Ziemiecki, A. S05-18, S05-19, S05-20

Ziemienovicz, A. S10-09

Zimmermann, D.R. S15-26

Zoñ, J. S13-06

Zrÿd, J.-P. S13-12, S13-14, S13-15

Zuber, H. S09-11, S15-47

Zückert, W.R. S20-13

Zufferey, R. S15-23

Ziuhlke, R.D. S12-01, S12-02

Zurbriggen, A. S04-24

Zurbriggen, R. S23-20

Zürcher, G. S05-18, S05-19, S05-20

Zurich, M.-G. S21-02

Zylicz, M. \$10-09 